\title{
Analysis of Compressive Fracture in Rock Using Statistical Techniques
}

Stephen Craig Blair

(Ph.D. Thesis)

December 1994

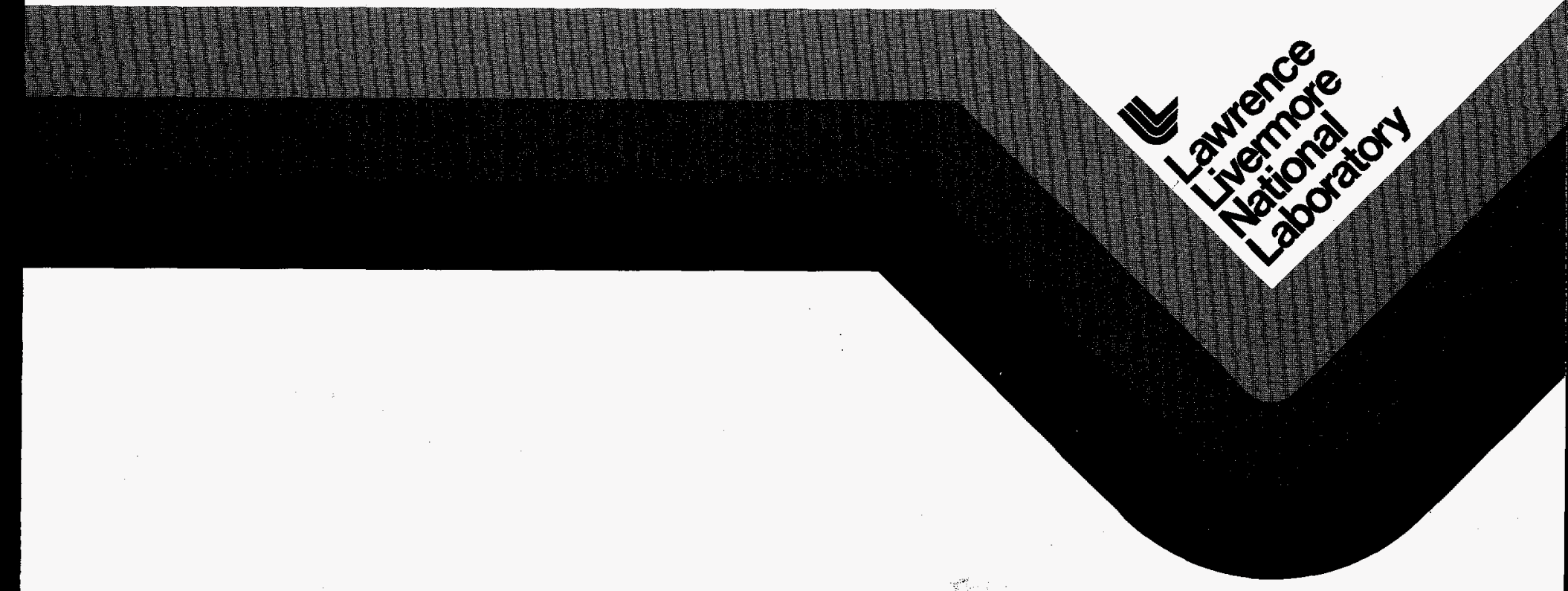




\section{DISCLAIMER}

This document was prepared as an account of work sponsored by an agency of the United States Government. Neither the United States Government nor the University of California nor any of their employees, makes any warranty, express or implied, or assumes any legal liability or responsibility for the accuracy, completeness, or usefulness of any information, apparatus, product, or process disclosed, or represents that its use would not infringe privately owned rights. Reference herein to any specific commercial products, process, or service by trade name, trademark, manufacturer, or otherwise, does not necessarily constitute or imply its endorsement, recommendation, or favoring by the United States Government or the University of California. The views and opinions of authors expressed herein do not necessarily state or reflect those of the United States Government or the University of California, and shall not be used for advertising or product endorsement purposes.

This report has been reproduced directly from the best available copy.

Available to DOE and DOE contractors from the Office of Scientific and Technical Information

P.O. Box 62, Oak Ridge, TN 37831

Prices available from (615) 576-8401, FTS 626-8401

Available to the public from the

National Technical Information Service

U.S. Department of Commerce

5285 Port Royal Rd.,

Springfield, VA 22161

Work performed under the auspices of the U.S. Department of Energy by Lawrence Livermore National Laboratory under Contract W-7405-Eng-48. 


\section{DISCLAIMER}

Portions of this document may be illegible in electronic image products. Images are produced from the best available original document. 


\title{
Analysis of Compressive Fracture in Rock Using Statistical Techniques
}

\author{
Stephen Craig Blair \\ (Ph.D. Thesis)
}

Manuscript date: December 1994

\section{LAWRENCE LIVERMORE NATIONAL LABORATORY}

University of California $\bullet$ Livermore, California $\bullet 94551$

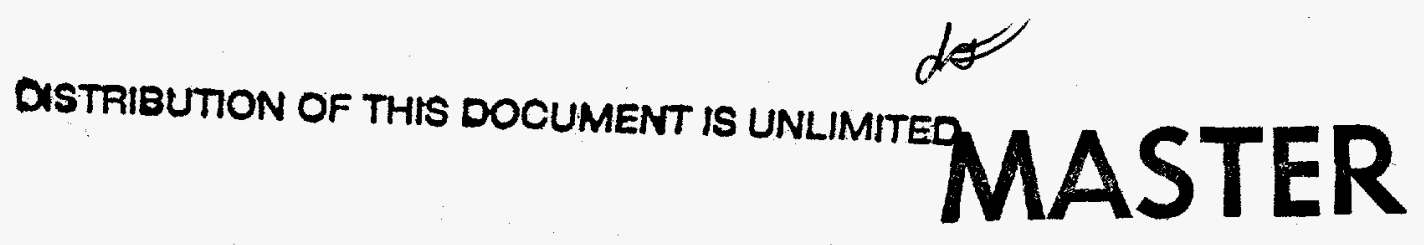


Analysis of Compressive Fracture in Rock

\title{
Using Statistical Techniques
}

\author{
by \\ Stephen Craig Blair
}

B.S. (University of California at San Diego) 1975 M.S. (University of Washington) 1980

A dissertation submitted in partial satisfaction of the requirements for the degree of

Doctor of Philosophy

in

Engineering-Materials Science

and Mineral Engineering

in the

GRADUATE DIVISION

of the

UNIVERSITY OF CALIFORNIA AT BERKELEY

Committee in charge:

Professor Neville G. W. Cook, Chair

Professor George A. Cooper

Professor Richard E. Goodman 
The dissertation of Stephen Craig Blair is approved:

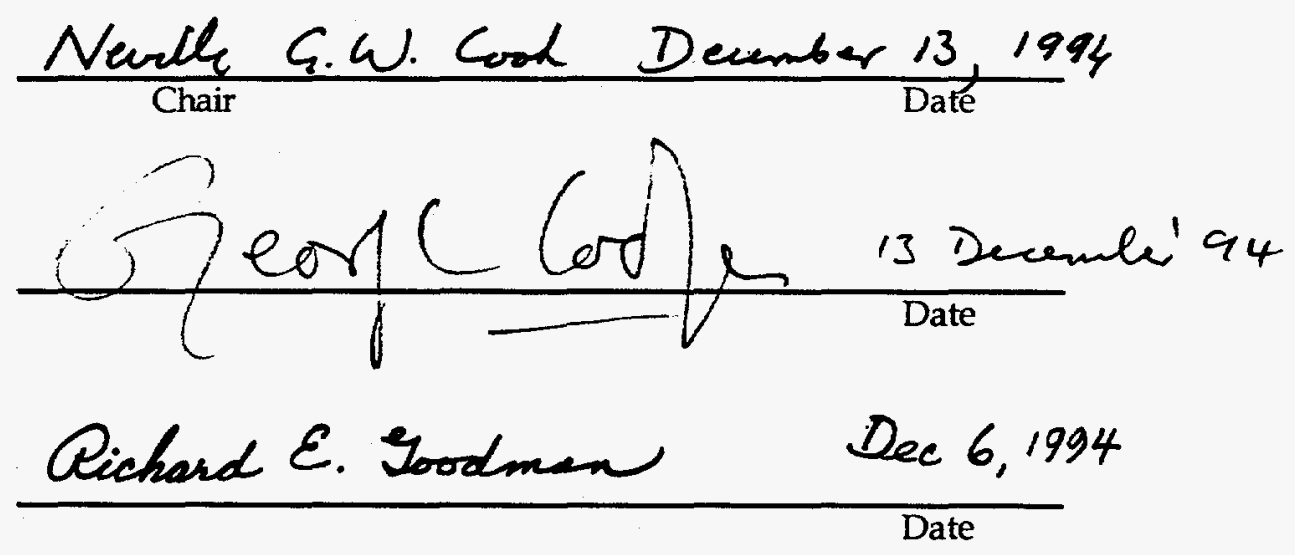

University of California at Berkeley 


\section{Dedication}

To my wife Fiona

and my children

Lukas, Katherine, and Jamie

for their support and understanding. 


\section{Contents}

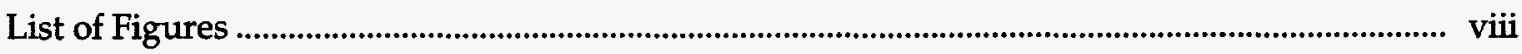

List of Tables ........................................................................................................................................... Xvi

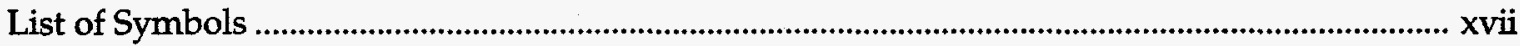

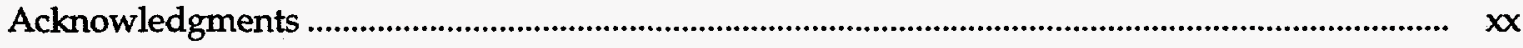

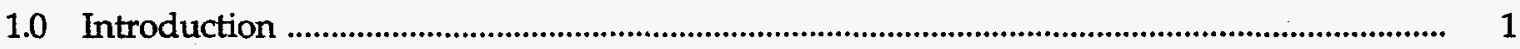

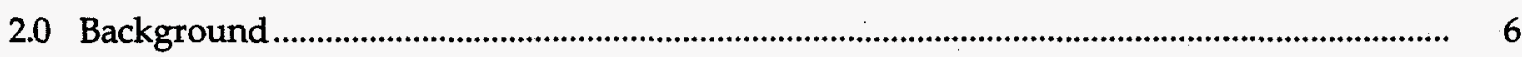

2.1 Experimental Observations of Rock Fracture ..................................................................... 6

2.2 Continuum Analysis and Linear Elastic Fracture Mechanics …...................................... 8

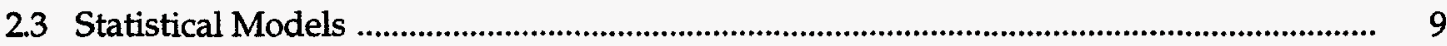

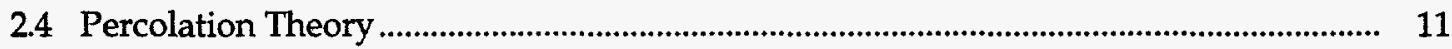

2.5 Application of Statistical Models to Rock Fracture .......................................................... 12

3.0 Nearest-Neighbor Model ............................................................................................... 15

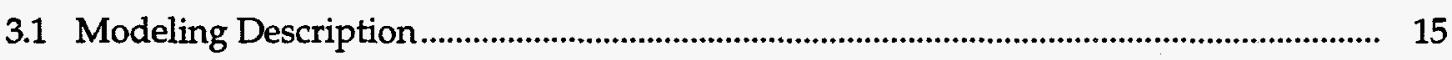

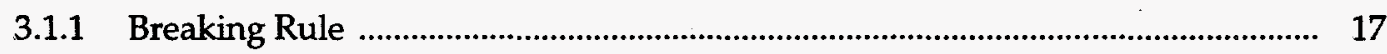

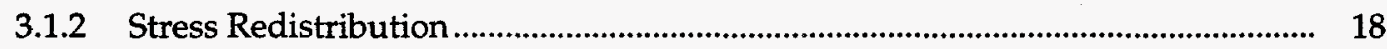

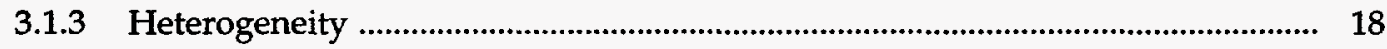

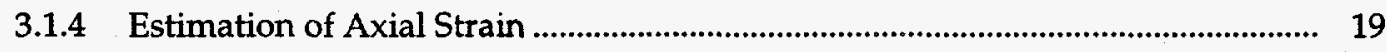

3.1.5 Crack Coalescence and Rock Fracture ....................................................... 21

3.2 Results-Simulation of Compression Tests .............................................................. 21

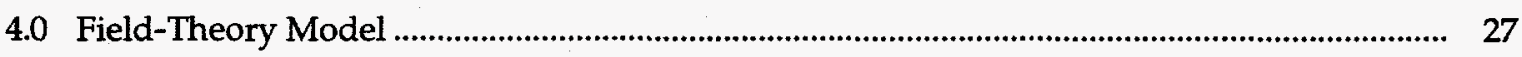

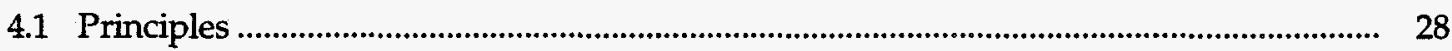

4.1.1 Overview of Concepts Used in Field-Theory Model ........................................... 28

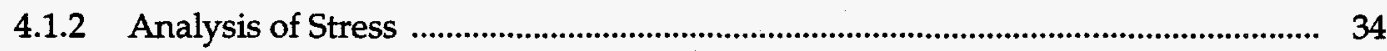

4.1.3 Introduction of Microscale Heterogeneity ..................................................... 36

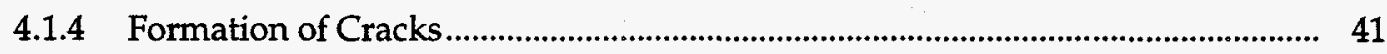


4.1.5 Computation of Strain

4.1.6 Changes in Boundary Conditions .................................................................... 50

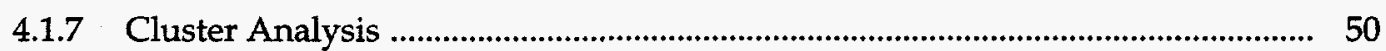

4.1.8 Relationship of Microscale Heterogeneity to Matrix Deformation Modulus .... 50

4.2 Model Implementation Using Field Theory and the Boundary Element Method ......... 52

4.2.1 Type and Number of Elements per Crack ............................................................ 53

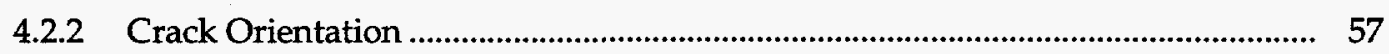

4.2.3 Computation of Strain Energy and Strain ..................................................... 59

4.3 Model Implementation on Lattices .................................................................................. 63

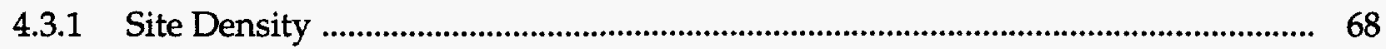

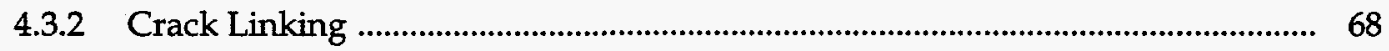

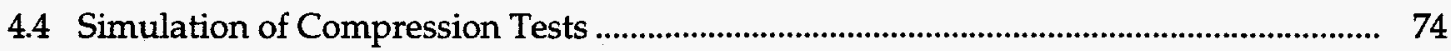

4.4.1 Computation of Macroscopic Stress-Strain Behavior ........................................... 76

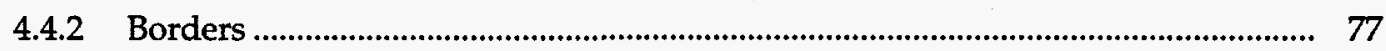

4.4.3 Boundary Conditions and Loading Path ….......................................................... 79

4.4.4 Failure Criterion …............................................................................................ 81

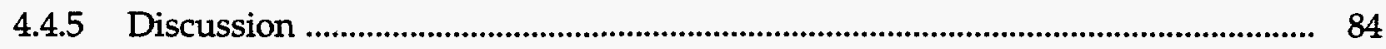

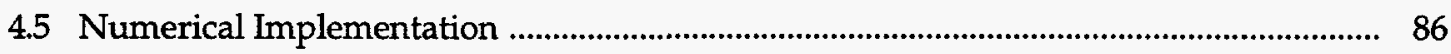

4.5.1 Programs astool and astool_batch ................................................................... 86

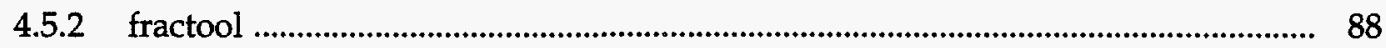

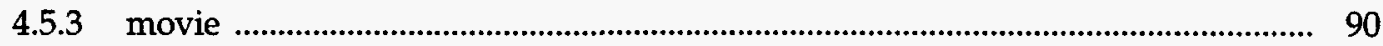

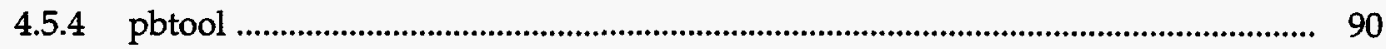

5.0 Results of Parameter-Sensitivity Analysis.......................................................................... 91

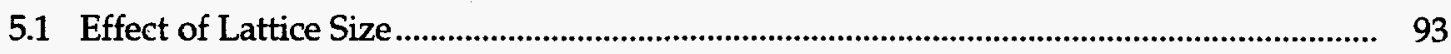

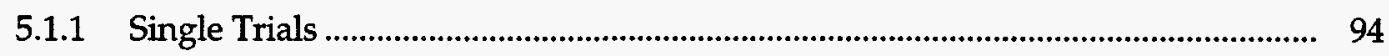

5.1.2 Multiple Trials_Ultimate Stress and Percolation Threshold ............................. 98

5.1.3 Discussion of Lattice Size Effect …......................................................................... 99 
5.2 Effect of Microscale Stress Heterogeneity: Triangular Lattice 105

5.2.1 Single Trials 105

5.2.2 Multiple Trials 108

5.2.3 Summary of Stress Perturbation Effect 114

5.3 Effect of Heterogeneity in Site Strength: Triangular Lattice. 116

5.3.1 Homogeneous Site Strength 118

5.3.2 Heterogeneous Site Strength 126

5.3.3 Bimodal Site Strength Distribution 136

5.3.4 Effect of Mean Site Strength 144

5.3.5 Summary of Site Strength Study on Triangular Lattice 147

5.4 Effect of Lattice Geometry-Perturbed Triangular Lattice 148

5.4.1 Homogeneous Site Strength Distribution 149

5.4.2 Heterogeneous Site Strength Distribution 158

5.4.3 Summary and Discussion for Perturbed Triangular Lattice 167

5.5 Effect of Heterogeneity in Site Strength: Square Lattice 169

5.5.1 Homogeneous Site Strength 169

5.5.2 Heterogeneous Site Strength 176

5.5.3 Summary of Site Strength Study on Square Lattice 177

5.6 Effect of Stress Heterogeneity on Square Lattice 183

5.7 Perturbed Square Lattice 185

5.7.1 Homogeneous Site Strength 185

5.7.2 Heterogeneous Site Strength 194

5.7.3 Summary and Discussion of Perturbed Square Lattice 195

5.8 Summary and Conclusions of Parameter Study 203

6.0 Analysis of Stress-Strain Behavior 206

6.1 Interrogation of Macroscopic Stress-Strain Behavior 206

6.2 Summary and Discussion 212 
7.0 Conclusions.

8.0 References 226

Appendix: Listing of Trials Computed for Parameter-Sensitivity Analysis . 233 


\section{List of Figures}

Figure 1.0-1. Cross section of Berea sandstone illustrating various types

of heterogeneity that occur in rock at the grain scale (from Berryman

and Blair, 1986)

Figure 3.1-1. (a) An array of uniform disks on a square lattice is used to represent the rock microstructure. Initially, boundary stresses are uniformly distributed on each disk. (b) Redistribution of $\sigma_{1}$ and $\sigma_{2}$ due to cracking of a disk. Stress increase is shown by increased arrow length (black), and stress decrease is shown by shortened arrow length

Figure 3.1-2. Stress-strain behavior for intact and cracked disks.

Cracked disks can support only a reduced amount of axial load.

Figure 3.1-3. Fracture is defined as occurring when a cluster of cracked disks spans the array. The perimeter above is divided into segments (A-F), and the array shows a fracture is formed when a cluster of cracked disks connects segments $B$ and F...

Figure 3.1-4. Logic of calculation

Figure 3.2-1. Stress-strain curves predicted for a 15 by 30 array at confining stresses of 10,20 , and $40 \mathrm{MPa}$

Figure 3.2-2. Locations of cracked disks for 2 points on the $20 \mathrm{MPa}$ confining stress curve shown in Figure 3.2-1: (a) locations of cracked disks at axial stress of $110 \mathrm{MPa}$; (b) locations of cracked disks at $150 \mathrm{MPa}$.

Figure 4.1-1. Stress-strain behavior associated with opening of a crack. Figures a-d are discussed in the text.

Figure 4.1-2. Decomposition of stress field into elastic continuum and cracked elastic body: (a) stress decomposition of body with an incipient crack, (b) stress decomposition used to form crack in $R$, (c) stress-strain response incorporating crack opening...

Figure 4.1-3. Stress field decomposition at site $i$ used in the fieldtheory model

Figure 4.1-4. Examples of localized tensile fracture in a compressive stress field: (a) a circular grain in diametric compression, (b) a tabular grain loaded in bending (adapted from Zheng, 1989).

Figure 4.1-5. Analysis of crack formation in the brazilian geometry using superposition. 
Figure 4.1-6. Detailed description of stress decomposition for crack formation in field-theory model

Figure 4.1-7. Illustration of annealing of $m_{1}$ parameter in field-theory

model: (a) initial stress field, (b) initial $m_{1}$ values for selected sites,

(c) stress field after a crack is introduced, (d) annealed $m_{1}$ values

Figure 4.2-1. Region where BEM solutions are inaccurate for three configurations

Figure 4.2-2. Maximum local tensile and compressive stresses computed for a simulated compression test vs number of elements used per cracked site.

Figure 4.2-3. Stresses applied to crack surface (after Crouch and

Starfield, 1990)

Figure 4.2-4. (a) Crack patterns at percolation threshold for 10 trials computed using a constant offset of $1^{\circ}$ clockwise for crack elements oriented along the vertical axis

Figure 4.2-4. (b) Crack patterns at percolation threshold for 10 trials computed using a stochastic offset algorithm.

Figure 4.3-1. Predicted crack patterns for regular and perturbed lattices

Figure 4.3-2. Production of perturbed square lattices: (a) Original sites are relocated to new coordinates.

Figure 4.3-2. (b) Sites at new coordinates become vertices of irregular quadrilaterals. Center of mass of quadrilaterals become sites on the perturbed lattice....

Figure 4.3-3. Production of perturbed triangular lattices: (a) original sites are relocated to new coordinates..

Figure 4.3-3. (b) Sites at new coordinates are connected to form a new

triangular grid. Center points of triangles on the new grid are located

Figure 4.3-3. (c) Center points are connected to form irregular hexagons.

The center of mass of each new hexagon becomes a site on the perturbed

lattice

Figure 4.3-4. Crack linking on a triangular lattice.

Figure 4.4-1. Schematic of geometry used in simulated compression

tests: (a) relative array dimensions, (b) boundary conditions. 
Figure 4.4-2. Effect of side border width on stress-strain behavior.

Figure 4.4-3. A cluster spans the lattice from B to E, but does not satisfy

kinematic failure criterion

Figure 4.5-1. Fracrock system of codes and files

Figure 4.5-2. Fracrock program outline for rock mechanics calculations

Figure 5.1-1. Effect of lattice size on stress-strain behavior for triangular lattices.

Figure 5.1-2. Effect of lattice size on relation of applied stress to normalized crack strain energy for triangular lattices.

Figure 5.1-3. Effect of lattice size on relation of fraction of cracked sites to crack strain energy for triangular lattices.

Figure 5.1-4. Crack patterns at the percolation threshold for triangular arrays with four different sizes. Shading displays horizontal stresses computed for array $S$, with light and dark shading representing tensile and compressive stresses respectively

Figure 5.1-5. Dependence of ultimate strength on lattice size for triangular lattices.

Figure 5.1-6. Dependence of percolation threshold on lattice size for triangular lattices.

Figure 5.2-1. Effect of changing the range of the stress difference multiplier on stress-strain behavior.

Figure 5.2-2. Effect of changing the range of the stress difference multiplier on the relation of applied stress to normalized crack strain energy

Figure 5.2-3. Effect of changing the range of the stress difference multiplier on the relation of number of cracked sites to normalized crack strain energy....

Figure 5.2-4. Crack patters at the percolation threshold for trials computed using four different ranges of the stress difference multiplier

Figure 5.2-5. Effect of changing the range of the stress difference multiplier, $m_{1}$, on mean ultimate strength

Figure 5.3-1. Examples of different site strength distributions used in this study: (a) $T_{g, i} 35 \pm 34.5 \mathrm{MPa}$, (b) $T_{g, i} 20 \pm 0.2 \mathrm{MPa}$, (c) $T_{g, i} 2 \pm 0.2$ $\mathrm{MPa}$ or $26 \pm 0.26 \mathrm{MPa}$. 
Figure 5.3-2. Stress-strain curves for 10 trials computed using

homogeneous site strength distributions

Figure 5.3-3. Relation of applied stress to normalized crack strain energy for 10 trials computed using homogeneous site strength distributions

Figure 5.3-4. Overstress ratio versus number of cracked sites at an applied stress of $38 \mathrm{MPa}$ for homogeneous site strength distribution. Values represent averages taken over 10 trials.....

Figure 5.3-5. Relation of number of cracked sites to normalized crack strain energy for 10 trials computed using homogeneous site strength distributions

Figure 5.3-6. Crack pattern development for 2 trials with homogeneous site strength distributions. Shading shows horizontal stress in $S$ array.

(a) crack pattern for trial \#4 123

Figure 5.3-6. (b) Crack pattern for trial \#5

Figure 5.3-7. Crack patterns at the percolation threshold for 10 trials computed using homogeneous site strength distributions

Figure 5.3-8. Stress-strain curves for 10 trials computed using heterogeneous site strength distributions.

Figure 5.3-9. Relation of applied stress to normalized crack stain

energy for 10 trials computed using heterogeneous site strength

distributions

Figure 5.3-10. Relation of overstress ratio to number of cracked sites at three different stress levels, for heterogeneous site strength distributions. Values are averaged over 10 trials

Figure 5.3-11. Relation of number of cracked sites to normalized crack strain energy for 10 trials computed using heterogeneous site strength distributions

Figure 5.3-12. Crack pattern development for 2 trials computed using heterogeneous site strength distributions: (a) crack patterns for trial \#4 132

Figure 5.3-12. (b) Crack patterns for trial \#10

Figure 5.3-13. Crack patterns at the percolation threshold for 10 trials computed using heterogeneous site strength distributions

Figure 5.3-14. Stress-strain curves for 10 trials computed using bimodal site strength distributions 
Figure 5.3-15. Relation of applied stress to normalized crack strain energy for 10 trials computed using bimodal site strength distributions

Figure 5.3-16. Relation of number of cracked sites to applied stress for

10 trials computed using bimodal site strength distributions.

Figure 5.3-17. Relation of overstress ratio to number of cracked sites at

2 levels of applied stress, for trials computed using bimodal site

strength distributions. Values are averaged over 10 trials.

Figure 5.3-18. Crack pattern development for two trials computed using

bimodal site strength distributions: (a) crack patterns for trial \#1

Figure 5.3-18. (b) Crack patterns for trial \#6.

Figure 5.3-19. Crack patterns at the percolation threshold for 10 trials

computed using bimodal site strength distributions.

Figure 5.3-20. Dependence of mean ultimate strength on mean site strength for trials computed using $m_{1}$ in two different ranges.

Figure 5.4-1. Effect of lattice perturbation on stress-strain curves for triangular lattice with homogeneous site strength distribution.

Figure 5.4-2. Effect of lattice perturbation on relation of applied stress

to normalized crack strain energy for triangular lattice with

homogeneous site strength distribution.

Figure 5.4-3. Effect of lattice perturbation on relation of number of cracked sites to normalized crack strain energy for triangular lattice with homogeneous site strength distribution

Figure 5.4-4. Effect of lattice perturbation on crack pattern

development for triangular lattice with homogeneous site strength

distribution. Crack patterns shown for one regular and two perturbed

trials, when 4,15 , and 30 sites are cracked, and at the percolation

threshold. $(a, b, c)$ Crack patterns when 4 sites are cracked.

Figure 5.4-4. (d, e, f) Crack patterns when 15 sites are cracked.

Figure 5.4-4. (g, h, i) Crack patterns when 30 sites are cracked.

Figure 5.4-4. (j, $k, 1)$ Crack patterns at or near the percolation

threshold

Figure 5.4-5. Effect of lattice perturbation on stress-strain curves for triangular lattice with heterogeneous site strength distribution 
Figure 5.4-6. Effect of lattice perturbation on relation of applied stress

to normalized crack strain energy for a triangular lattice with

heterogeneous site strength distribution

Figure 5.4-7. Effect of lattice perturbation on relation of number of

cracked sites to normalized crack strain energy for triangular lattice

with heterogeneous site strength distribution.

Figure 5.4-8. Effect of perturbation of triangular lattice on crack

patterns for trials computed using a heterogeneous site strength

distribution: $(a, b, c)$ Crack patterns for 25 cracked sites ........................................ 162

Figure 5.4-8. (d, e, f) Crack patterns when 45 sites are cracked ................................... 163

Figure 5.4-8. $(\mathrm{g}, \mathrm{h}, \mathrm{i})$ Crack patterns when 65 sites are cracked........................................ 164

Figure 5.4-8. $(j, k, 1)$ Crack patterns at percolation threshold.......................................... 165

Figure 5.5-1. (a) Stress-strain curves for 10 trials computed using a

square lattice and homogeneous site strength distribution.

Figure 5.5-1. (b) Stress-strain curves for 9 of the trials shown in

Figure 5.5-1a.

Figure 5.5-2. Relation of applied stress to normalized crack strain

energy for 10 trials computed using homogeneous site strength

distributions on a square lattice

Figure 5.5-3. Relation of number of cracked sites to normalized crack

strain energy for 10 trials computed using a homogeneous site strength

distributions on a square lattice

Figure 5.5-4. Crack pattern development for trial \#9, homogeneous site

strength distribution on a square lattice

Figure 5.5-5. Crack patterns at the percolation threshold for 10 trials

computed on a square lattice, using homogeneous site strength

distributions

Figure 5.5-6. Stress-strain curves for 10 trials computed using

heterogeneous site strength distributions on a square lattice

Figure 5.5-7. Relation of applied stress to normalized crack strain

energy for 10 trials computed using heterogeneous site strength

distributions on a square lattice

Figure 5.5-8. Relation of number of cracked sites to normalized crack

strain energy for 10 trials computed using heterogeneous site strength

distributions on a square lattice 
Figure 5.5-9. Crack pattern developnent for two trials with heterogeneous site strength distribution on a square lattice: (a) crack pattern for trial \#7

Figure 5.5-9. (b) Crack patterns for trial \#8

Figure 5.5-10. Crack patterns at the percolation threshold for 10 trials

computed on a square lattice, using heterogeneous site strength

distributions

Figure 5.6-1. Dependence of ultimate strength on $m_{1}$ for square and triangular lattices. Open and solid symbols represent homogeneous and heterogeneous site strength distributions, respectively

Figure 5.7-1. Effect of perturbation of a square lattice on stress-strain curves for trials computed using a homogeneous strength distribution

Figure 5.7-2. Effect of perturbation of a square lattice on relation of applied stress to normalized crack strain energy for trials computed using a homogeneous site strength distribution.

Figure 5.7-3. Effect of perturbation of a square lattice on relation of number of cracked sites to normalized crack strain energy for trials computed using homogeneous site strength distribution

Figure 5.7-4. Effect of perturbation of a square lattice on crack patterns for trials computed using a homogeneous site strength distribution: $(a, b, c)$ Crack patterns for 8 cracked sites 189

Figure 5.7-4. (d, e, f) Crack patterns for 18 cracked sites. 190

Figure 5.7-4. (g, h, i) Crack patterns for 30 cracked sites 191

Figure 5.7-4. (j, k, l) Crack patterns for 40 cracked sites. 192

Figure 5.7-4. $(m, n, o)$ Crack patterns at percolation threshold. 193

Figure 5.7-5. Effect of perturbation of a square lattice on stress-strain curves for trials computed using a heterogeneous site strength distribution.

Figure 5.7-6. Effect of perturbation of a square lattice on relation of applied stress to normalized crack strain energy for trials computed using a heterogeneous site strength distribution.

Figure 5.7-7. Effect of perturbation of a square lattice on relation of number of cracked sites to normalized crack strain energy for trials computed using a heterogeneous site strength distribution 
Figure 5.7-8. Effect of perturbation of a square lattice on crack patterns:

(a, b, c) Crack patterns for 25 cracked sites.

Figure 5.7-8. (d, e, f) Crack patterns for 45 cracked sites.

Figure 5.7-8. $(g, h, i)$ Crack patterns for 80 cracked sites

Figure 5.7-8. (j, $k, 1)$ Crack patterns at or near the percolation

threshold

Figure 6.1-1. Stress-strain curve for $30 \times 90$ array

Figure 6.1-2. Relation of applied stress to normalized crack strain

energy for $30 \times 90$ array

Figure 6.1-3. Relation of number of cracked sites to normalized crack

strain energy for $30 \times 90$ array

Figure 6.1-4. Relation of overstress ratio to number of cracked sites at a given stress level for $30 \times 90$ array

Figure 6.1-5. Crack patterns for $30 \times 90$ array

Figure 6.1-6. Relation of overstress ratio to number of cracked sites at an applied stress of $14 \mathrm{MPa}$

Figure 6.1-7. Relation of overstress ratio to number of cracked sites for intervals $c$ and $d$ of stress-strain curve for $30 \times 90$ array 


\section{List of Tables}

Table 4.1-1. Dependence of deformation modulus on heterogeneity in the matrix

Table 4.3-1. Crack linking. 72

Table 4.4-1. Boundary conditions for simulation of compression tests.

Table 4.5-1. Codes and functions used in Fracrock model

Table 5.0-1. Summary of parameters used in sensitivity analysis 92

Table 5.1-1. Summary data for single trials

Table 5.1-2. Ultimate strength and percolation thresholds for simulations with different lattice sizes. All trials were at baseline conditions with $p_{c}=0.1, m_{1}$ in \pm 0.7 .

Table 5.2-1. Data for single trials for different ranges of $m_{1}$ 106

Table 5.2-2. Summary data for $m_{1}$ trials.

Table 5.3-1. Dependence of ultimate strength on mean site strength for uniaxial simulations using $10 \times 30$ arrays

Table 5.4-1. Data for single trials for perturbed triangular lattice. 168

Table 5.5-1. Summary data for square lattices 183

Table 5.6-1. Summary of trials for square arrays. 185

Table 5.7-1. Data for single trials for perturbed square lattice. 202

Table 5.7-2. Mean values of ultimate strength and percolation threshold for perturbed square lattice. $m_{1}$ in \pm 0.7 for all trials 


\section{List of Symbols}

$\alpha$

$\beta_{i}$

$\delta_{i}$

$\varepsilon$

$\eta$

$v$

$\Pi$

$\phi_{i}$

$\rho$

$\rho_{i}$

$\sigma$

$\sigma_{t}$

$\sigma_{1}$

$\sigma_{2}$

$\sigma$

$\left(\sigma_{1, i}\right)_{E},\left(\sigma_{2, i}\right)_{E} \quad$ Local maximum, minimum principal stress components at site $i$ in $E$.

$\left(\sigma_{1, i}\right)_{S},\left(\sigma_{2, i}\right)_{S}$

$\sigma_{\mathrm{I}}$

$\sigma_{\text {II }}$

$\left(\sigma_{c, i}\right)_{S}$

$-\sigma_{c, i}$

$\sigma_{n s}$

$\left(\sigma_{\theta, i}\right)_{R}$

Constant.

Direction of local maximum principal stress at site $i$.

Crack aperture of crack at site $i$.

Strain.

Critical exponent in connectedness correlation function.

Critical exponent in connectedness correlation function.

Summation of $\rho$.

Direction of local minimum principal stress at site $i$ in $E$.

Stress perturbation at a site in the uncracked elastic continuum

representative of geometric variation in the material. This term sums to zero over entire array and incorporates stress difference at the site.

Stress perturbation of site $i$.

Stress.

Tensile stress in a grain.

Maximum principal stress.

Minimum principal stress.

Perturbed stress.

Local maximum, minimum principal stress components at site $i$ in $S$.

Applied maximum principal stress.

Applied minimum principal stress.

Crack-opening stress in $S$.

Crack traction.

Stress at a site in a cracked elastic body in direction perpendicular to $\left(\sigma_{1, i}\right)_{E}$ at that site. This is due to crack interaction only.

Stress along direction $\theta$ at site $i$ in $R$. 


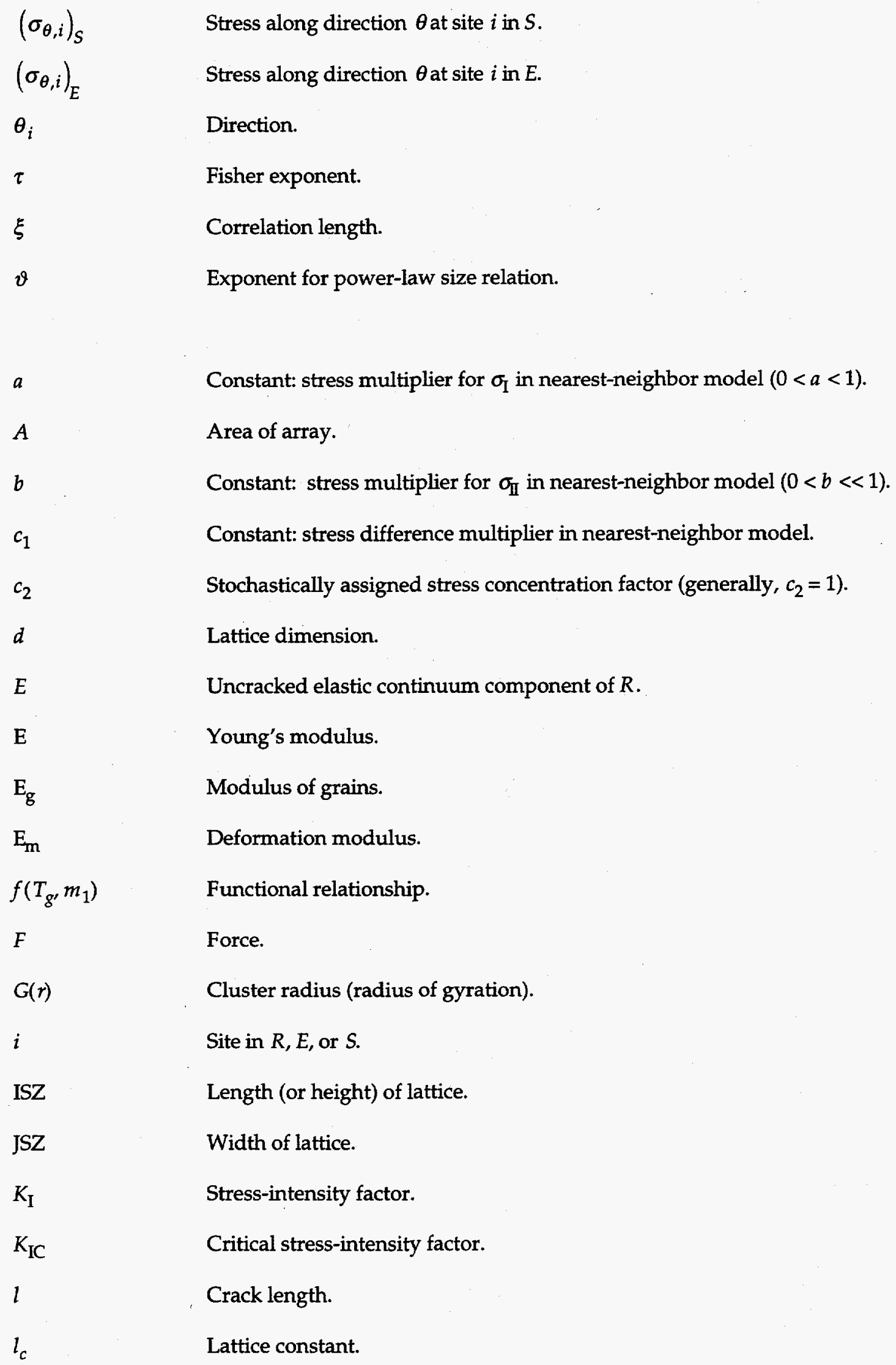




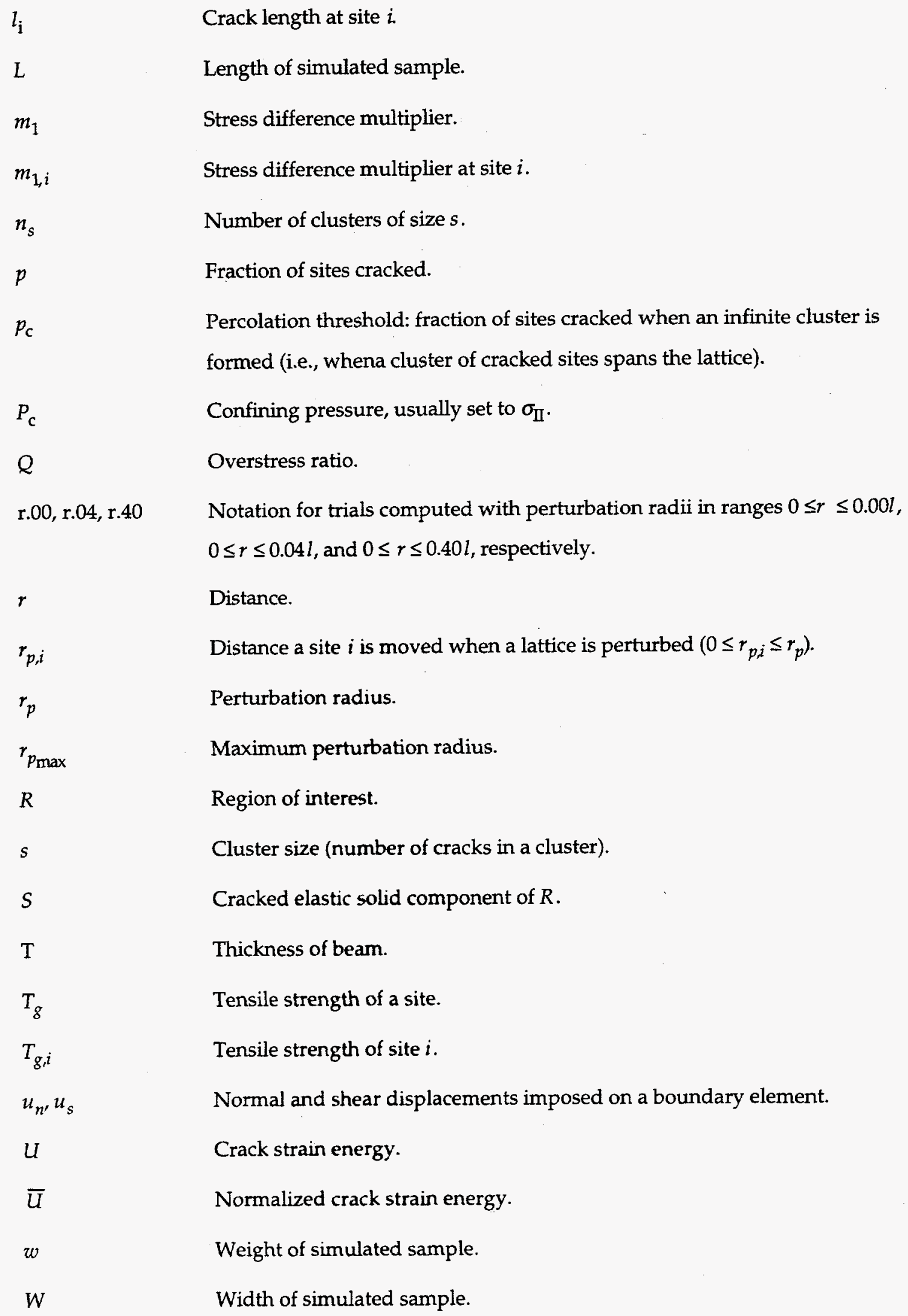




\section{Acknowledgments}

This work would not have been possible without the guidance of Professor Neville Cook, who has mentored my technical and professional growth through this work, and his patience and insight into the work and his careful attention to detail in the formulation and analysis are greatly appreciated.

Stephanie Daveler at LLNL deserves special acknowledgment for the exceptional level of support she provided in programming the many iterations of the computational and display codes that form the model, and for assisting in the setup and execution of the trials used in the dissertation. Stephanie also assisted in the production of many of the final figures used in Chapters 4,5, and 6. This work would not have been possible without her deft programming capability, patience, and attention to detail.

I also want to express my sincerest gratitude to Cynthia Talaber at LLNL, who assisted in organizing, typing, and other aspects of preparation of the manuscript.

John Kelly participated in the summer teacher program at LLNL and assisted in the organization of the many trials and set up a database program that allows various parameters to be queried from the overall data set.

I also want to acknowledge the members of my committee, Profs. G. E. Cooper and R. E. Goodman, for their insightful and timely reviews and suggestions on the manuscript, and Dr. Michael Hood, who chaired my qualifying exam committee.

I want to acknowledge the management and staff of the Earth Sciences Division and the Employee Development Division at LLNL for their moral and financial support during my tenure as a graduate student. In particular, I thank my superiors Jim Berryman, Lee Younker, Dale Wilder, and Al Duba for providing me the opportunity and constant encouragement in my participation in this graduate program.

Acknowledgment is also due to the many students and colleagues at UC Berkeley, LLNL, and LBL who have enriched my life and set a high technical standard for this study. These 
include: Patricia Berge, Ken Lee, Armand Attilla, John Rundle, Brian Bonner, Bill Durham, Tony Ladd, Kurt Nihei, Brun Hilbert, Larry Myer, Laura Pyrak-Nolte, Z. Zheng (ZZ), Russ Ewy, John Kemeny, Sharad Kelkar, Stacey Ita, Galen Hesler, Roberto Suarez, and Sanjit Roy.

Not to be forgotten are my parents, Melvin and Doris Blair, who have offered constant encouragement and interest in the completion of this work, and friends John and Ardath Schaibly and Phil and Pat Williams who have encouraged and assisted my wife Fiona and me during the production of the thesis.

I gratefully acknowledge the support of the Geosciences Program in the Office of Basic Energy Sciences and of the Near-Field Environment area within the Yucca Mountain Project.

Finally, a very special acknowledgment and thank-you to my wonderful wife, Fiona Doyle, for her caring and encouragement and technical advice on this effort. She has given me two wonderful children during the completion of this project and endured a solid year of weekends and evenings as a single parent while I completed this work and has been a source of constant encouragement and inspiration. 


\begin{abstract}
Fracture of rock in compression is analyzed using a field-theory model, and the processes of crack coalescence and fracture formation and the effect of grain-scale heterogeneities on macroscopic behavior of rock are studied. The model is based on observations of fracture in laboratory compression tests, and incorporates assumptions developed using fracture mechanics analysis of rock fracture. The model represents grains as discrete sites, and uses superposition of continuum and crack-interaction stresses to create cracks at these sites. The sites are also used to introduce local heterogeneity. Clusters of cracked sites can be analyzed using percolation theory.

Stress-strain curves for simulated uniaxial tests were analyzed by studying the location of cracked sites, and partitioning of strain energy for selected intervals. Results show. that the model implicitly predicts both development of shear-type fracture surfaces and a strength-vs-size relation that are similar to those observed for real rocks. Moreover, results show that upon initial loading, cracking is controlled by grain-scale heterogeneity and cracks are randomly located. When approximately $10 \%$ of the sites are cracked, crack interaction becomes important, and crack clusters form and extend. This may be interrupted by crack arrest and intervals of random cracking. Finally, major clusters are connected by a few critical connecting cracks. This may be equivalent to unstable crack extension. The macroscopic crack surfaces formed resemble those formed by shear localization in real rock.

Results of a parameter-sensitivity analysis indicate that heterogeneity in the local stresses, attributed to the shape and loading of individual grains, has a first-order effect on strength, and that increasing local stress heterogeneity lowers compressive strength following an inverse power law. Peak strength decreased with increasing lattice size and decreasing mean site strength, and was independent of site-strength distribution. Results also indicate that stepping of cracks observed in fractured rock may be due to heterogeneity in grain packing.

A model for rock fracture based on a nearest-neighbor algorithm for stress redistribution is also presented and used to simulate laboratory compression tests, with promising results.
\end{abstract}




\title{
Analysis of Compressive Fracture in Rock Using Statistical Techniques
}

\author{
Stephen Craig Blair
}

\subsection{Introduction}

The mechanical behavior of rock has been studied at least since the time of the early Egyptians, who developed engineering methods to excavate rock for the pyramids. Despite extensive work since these early developments, several aspects of the mechanical behavior of rocks remain poorly understood. One of the most important areas in which additional understanding is needed concerns the fundamental processes of deformation and fracture of rock. An understanding of these processes is important in maintaining the integrity of engineered excavations and in processes such as communition ${ }^{1}$ and drilling where fracture or breakage is desired.

Rock is a heterogeneous material and is known to fracture via the formation, growth, and coalescence of microcracks. Further, experimental observations of rock tested in compression have shown that many of the microcracks occur at the grain scale as mode I extensile cracks oriented in the direction perpendicular to the least principal stress. The formation of these

\footnotetext{
1 An improved understanding of rock fracture is important to the broad interests of the global society, as it could lead to significant energy savings on a national and worldwide scale. This is because approximately $4 \%$ of the world's total energy output is used in the communition (fracture) of rock (Kapur, 1993). Moreover, this process has a very low efficiency (approximately $1.1 \%$; Kapur, 1993). Better understanding of rock fracture and how fracture processes scale with size could lead to more efficient communition techniques and a reduction in worldwide energy consumption.
} 
dilatant cracks in a compressive stress field is attributed to the existence of local regions of tension at the grain scale due to heterogeneity in the shape and loading geometry of the individual grains (Gallagher et al., 1974).

Moreover, the exact nature of the effect of microscale heterogeneity in the shape and loading of grains in the fracture process is poorly understood. The discontinuous nature and the heterogeneity present at the grain scale in rock is illustrated in Figure 1.0-1, which shows a cross section of a sample of Berea sandstone (Berryman and Blair, 1986). This image shows the sandstone to be a two-phase medium with bright grains and dark pores. Several different types of grain-scale heterogeneity are easily observed, including heterogeneity in the size and shape of grains, the type and geometry of grain contacts, the grain mineralogy, and the amount of weathering. Moreover, even though this cross section was prepared from an intact sample, a few of the grains are cracked. This brief discussion shows that even a "uniform" rock such as Berea sandstone has significant heterogeneity at the grain scale, and how this heterogeneity can affect grain-scale cracking and associated changes in the macroscopic properties.

A second aspect of rock fracture that is not well understood is the dynamic evolution of the fracture process. That is, while it is generally acknow'ledged that rock fracture is due to the formation, growth, and coalescence of cracks and mucrocracks, the role of crack interaction in the process is poorly known. One of the central questions is: at what point does crack interaction overwhelm the local variations in the stress field, or variations in other local properties, and drive the system to fracture either by coalescence of cracks or by extension of one crack?

Statistical modeling is a promising technique for addressing these problems. In a statistical model the basic elements of a material are represented by sites or bonds, and behavior of the sites (bonds) is governed by basic laws or rules. The macroscopic material is then modeled by constructing a lattice of sites (bonds). Using this approach the dynamic evolution of the behavior of a system can be observed directly, and is a consequence of the rules used to govern the basic elements. This is in contrast to the continuum approach, which tries to describe behavior of a parameter field by using the "best" mathematical approximation to that field. Statistical models 


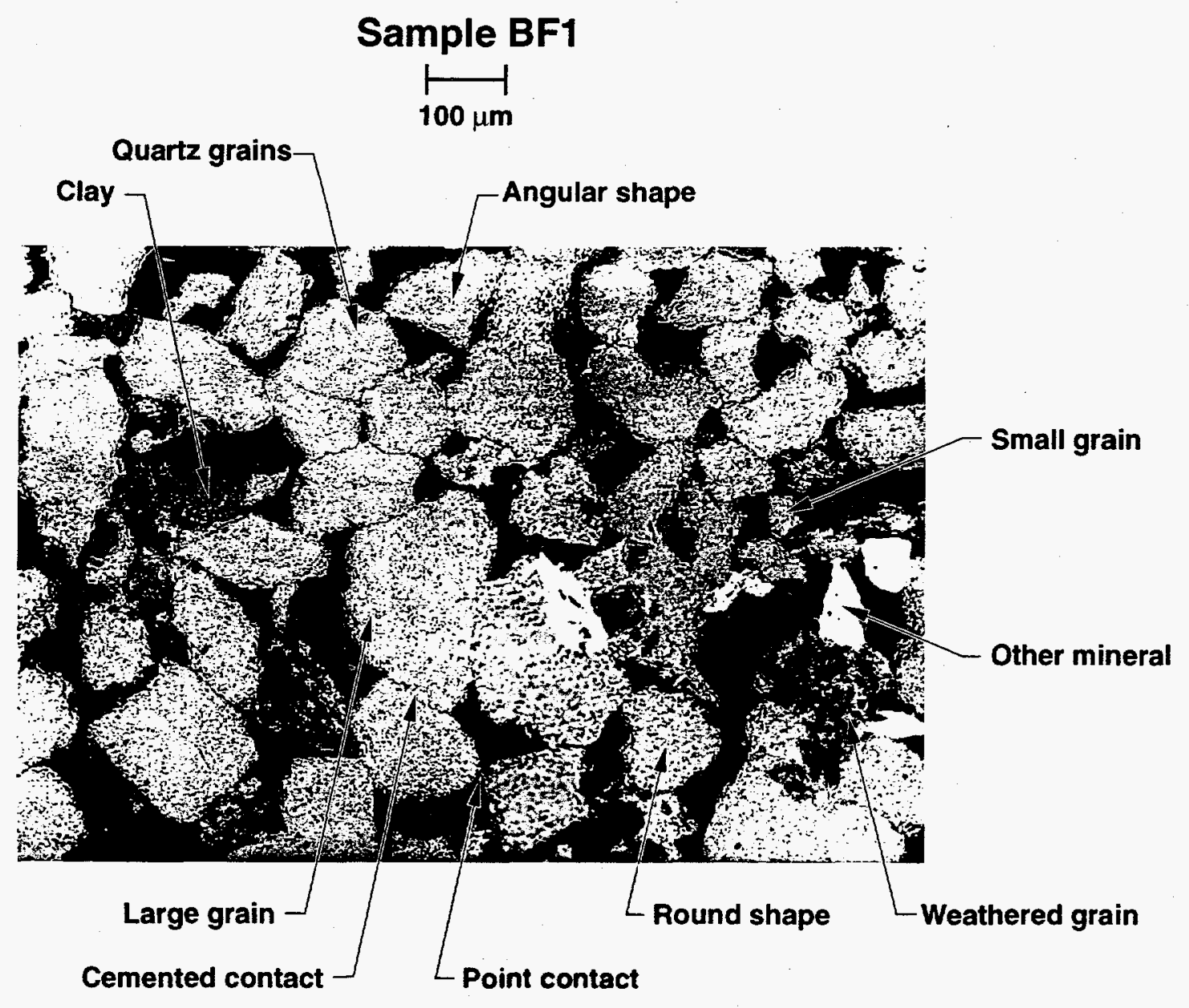

Figure 1.0-1. Cross section of Berea sandstone illustrating various types of heterogeneity that occur in rock at the grain scale. (from Berryman and Blair, 1986) 
have been used by the statistical mechanics and statistical physics communities to study behavior of several idealized systems. These models have also been used to study fracture in rock and other heterogeneous materials, and in this case the sites or bonds are usually taken to represent grains.

Statistical models are also well suited for studying fracture in rock and other heterogeneous media, as they can easily incorporate heterogeneity because they are rule-based. Thus, if a site or a bond is used to represent a grain in a rock, it can be assigned a tensile strength, and if the tensile stress computed at that site exceeds the tensile strength, the site (bond) can be "broken" by changing its state or removing it from the lattice. In addition, the rules governing the sites can be easily changed, making the models very flexible. In addition, these models generally have fewer unknowns than continuum models, making it easier to isolate the effects of a particular parameter.

Statistical models also have the advantage that they can be used in conjunction with percolation theory to develop and study scaling relations. This provides a technique for analyzing the evolution of the fracture process by using the number and spatial distribution of the broken sites to estimate universal exponents, which are parameters that can be used to describe general classes of behavior.

While statistical models hold a great deal of promise for study of fracture in rock and other heterogeneous materials, it is important to realize that they are in their infancy with respect to the study of rock fracture in compression. First of all, most of the applications to date have been to theoretical problems, studying ideal systems that generally are not similar to rock. Moreover, most of this work has simulated fracture in tension, while most important rock mechanics problems involve compression (i.e., rock behavior in the subsurface). Further, the range of inputs for properties of sites and bonds used to represent grains in rock is largely unknown, and much of the work to date has focused on the effect of heterogeneity in site strength. This may be irrelevant if real rock fracture is dominated by other microscale parameters such as grain shape. 
The objective of this dissertation is to develop a model for rock fracture in compression that incorporates a few basic assumptions regarding the fracture of rock in compression along with selected attributes of grain-scale heterogeneity. The model must also provide access to percolation analysis of the fracture process. The expectation is that such a model will provide fresh insight into both the effect of grain-scale heterogeneity on fracture and the dynamic evolution of the fracture process.

Chapter 2 of this dissertation presents background information on fracture of rock, along with a discussion of the importance of heterogeneity and of possible methods of analysis. In Chapters 3 and 4 two different numerical models for rock fracture in compression are presented. Chapter 3 presents a nearest-neighbor model that is similar to a cellular automaton. This model is used to simulate uniaxial and triaxial laboratory compression tests, and results are presented and discussed. A field-theory model is presented in Chapter 4 that incorporates the concept of superposition, and the techniques of boundary element analysis (Crouch and Starfield, 1990) and percolation theory (Stauffer, 1985), to form a simple yet powerful method for studying progressive fracture of rock and other heterogeneous materials. The field-theory model was used to conduct a sensitivity analysis to assess how heterogeneity at the grain scale in site strength and local geometry affects macroscale mechanical behavior such as stress-strain; results of the parameter study are presented in Chapter 5. The field-theory model was also used to interrogate stress-strain behavior in terms of the evolution of crack formation, extension, and coalescence to gain insight into the process of fracture in compression; results of this analysis are presented in Chapter 6. Chapters 7 and 8 present conclusions and references, respectively. 


\subsection{Background}

A great deal of work has been done experimentally to describe the process of deformation and fracture of rock in compression, and many of the observations and results from these studies have been incorporated into continuum fracture mechanics models for rock behavior. However, at the micromechanical scale, most rocks are a complex assemblage of crystals or grains, cementing material, and, in many cases, clays and overgrowths; the experimental results indicate that the overall strength and the static and dynamic properties of the rock are controlled by both the spatial arrangement of the grains and their microgeometry, and by the mechanical properties of the individual grains and cementing materials. Thus, for rocks with a moderate amount of heterogeneity at the grain scale, the continuum approach is not well suited for analysis of fracture processes.

Statistical models present an alternative to the continuum models; recently these models have been adapted to study rock fracture in compression. These models have advantages in that they can easily incorporate heterogeneity and that results can be analyzed using percolation theory, which provides a methology for development of universal scaling relations to describe rock behavior.

In this chapter the relevant experimental work and continuum analyses are reviewed and the concepts of statistical models and percolation theory are introduced. Finally, recent applications of statistical models to rock fracture are reviewed

\subsection{Experimental Observations of Rock Fracture}

Experimental observations have provided a great deal of insight into the relationship between the complex macroscopic deformation and failure of rock under differential compressive stresses and the microscopic processes that give rise to this behavior. Stress-strain measurements in conjunction with observation of the changes in microstructure during the stress-strain test have been instrumental in understanding rock deformation and fracture in the brittle field. Brace 
et al. (1966) and others (e.g., Wawersik and Fairhurst 1970, Wawersik and Brace, 1971;

Bieniawski, 1976; Brace, 1971; and Hallbauer et al., 1973) have made detailed measurements of the stress-strain behavior of rock in compression and have divided stress-strain curves for brittle rocks measured in laboratory tests into several regions, each of which is dominated by a different mode of crack behavior.

A key concept developed from these and other observations is that heterogeneities in the microstructure produce local concentrations of tensile stress, even when the rock as a whole is subjected only to compressive stresses (Gallagher et al, 1974). These local tensile stresses result in extensile microcracking. Experimental observations of microcrack growth under differential compression have been made by Wawersik and Brace (1971), Peng and Johnson (1972), Hallbauer et al. (1973), Olsson (1974), Kranz (1979), Batzle et al. (1980), Wong (1982), Fredrich and Wong (1986), and others. These observations have confirmed that opening mode I extensile microcracking occurs parallel to the direction of the maximum compressive stress, that there are several mechanisms responsible for the local tensile stress concentrations and the formation and growth of cracks and microcracks, and that some mechanisms such as bending can cause very high local stress concentrations.

Measurements of displacement, pore volume change (Brace, 1978; Zhang et al., 1990), acoustic emission (Zhang et al., 1990; Lockner et al., 1991), and seismic velocities and attenuation (Hadley, 1976; Holt and Fjaer, 1987) have been used to infer the mechanisms of deformation and failure in crystalline and clastic rocks for a variety of loading conditions. Zheng et al. $(1987,1989)$ adapted the Wood's metal porosimetry technique to preserve microstructure as it exists under load. This technique has enabled visual (aided by optical and SEM microscopy) examination of the microstructure accompanying failure in solid circular cylinders (Zheng, 1989), hollow cylinders (Ewy and Cook, 1990), and under indentation (Suarez-Rivera et al., 1990). More recently, Zhao et al. (1993) examined fracture under SEM.

In summary, laboratory studies have shown that in compression rocks fracture via the formation, extension, and coalescence of extensile cracks. These cracks are generally oriented in 
the direction of the maximum principal stress and as more cracks are formed or existing cracks grow, crack interaction becomes important. The formation and extension of these cracks under conditions of compression is attributed to local tensions at the microscale that may be caused by heterogeneity in the shape, orientation, and strength of grains, and other factors. Moreover, Lockner et al. (1991) show that under servo-controlled loading conditions, shear localization starts only after the rock has reached its peak strength and had already started to strain-soften, and Myer et al. (1992) have shown that extensile microcracking is not limited to situations in which stress conditions are uniform, such as in a compression test of a cylinder of rock, but also occurs under general loading conditions such as those around a borehole or beneath the indentor of a drill bit.

\subsection{Continuum Analysis and Linear Elastic Fracture Mechanics}

Many of the relationships between growth and interaction of cracks and the deformation and strength of rock can be analyzed using linear elastic fracture mechanics. Rudnicki and Rice (1975) treated the onset of shear localization as an instability in the description of homogeneous deformation, and showed that the onset of shear localization can be related to the onset of nonunique solutions in the homogeneous stress-strain relations. Other investigators have developed specific mechanisms or models for microcracks that are based on experimental observations, and have implemented these using fracture mechanics techniques. These include stress concentrations around pores (Sammis and Ashby, 1986), elastic mismatch between grains (Dey and Wang, 1981), Hertzian contact (Zhang et al., 1990), grain bending (Gallagher et al., 1974), dislocation pile-up (Horii and Nemat-Nasser, 1986; Wong, 1990), and sliding along preexisting cracks (Horii and Nemat-Nasser, 1985, 1986; Ashby and Hallam, 1986; Kemeny and Cook, 1987, 1991). Stress-intensity-factor solutions have been developed for many of these. By combining the effects of microcracks on effective moduli with the stresses required to cause microcrack propagation, it is possible to analyze the nonlinear stress-strain relationships for rock, including strain-hardening, strain-softening, dilatation, and localization. This approach has been 
particularly successful in modeling the behavior of crystalline rock, which can be thought of as a continuum with porous inclusions in the form of low-aspect-ratio cracks.

Of particular interest is the generic crack model of Kemeny and Cook (1991). These authors reviewed many of the fracture mechanics models presented above and formulated a generic model for cracking that provides that the stress-intensity factor is proportional to the local stress difference $\left(\sigma_{1}-\sigma_{2}\right)$ at the particular site.

It is important to note that many of the phenomena observed in experiments that influence the deformation and the fracture of rock are not amenable to analysis using a continuum approximation, and that further analyses of the micromechanics of the deformation and failure of porous clastic rocks will require explicit recognition of their discontinuous and granular nature. Moreover, to incorporate this type of heterogeneity into a continuum model is computationally intensive. This is because for a continuum model to accurately incorporate heterogeneity, the scale of the discretization must be much more refined than the scale of the heterogeneity of interest. In addition, the standard continuum methods have no capacity for developing a generalized scaling relation. Thus, fracture mechanics works well when the number of fractures and the amount of heterogeneity are restricted. However, the laboratory experiments discussed above show that the grain-scale processes that lead to macroscale deformation and fracture are very localized and dependent on the stochastic nature of the grain-scale heterogeneity.

\subsection{Statistical Models}

Statistical modeling has emerged as a powerful technique to study fracture processes in rock and other heterogeneous materials. It can be used to construct ideal systems in which all physical properties are known; this allows the effects of heterogeneity in grain microstructure and grain properties on the macroscopic rock properties to be examined in a quantitative way. Repeated simulation can be used to isolate the effects of a particular microstructural feature such as grain size or strength, and a simulation can be stopped at any stage in the analysis to examine the local 
stresses and displacements of individual elements within the simulated rock sample. Models of this type are often classified as physical or statistical models.

In a statistical model the body of interest is simulated using a lattice or a network of sites, bonds, or both. The behavior of each site or bond is governed by simple, one-dimensional rules. For instance, a material may be represented by a network of springs, and each spring is assigned a modulus and a breaking strength. Stress or displacement is then applied to the boundaries and the network is solved to determine the stress in each spring. If for a particular spring the stress exceeds the strength assigned to the spring, it is "broken" and removed from the network. Lattices of sites can be used in the same manner, or a network of sites and bonds can be constructed where both sites and bonds have properties. This approach provides a way to study complex systems using simple elements. Using simple brittle-bond elements this type of model can produce globally ductile behavior for a network where material shows strain-softening.

One of the major advantages of a statistical model for studying fracture in heterogeneous materials such as rock is that local heterogeneity can be incorporated very easily. For instance, to simulate a material made of grains with varying strength, the bonds in the lattice can be assigned strengths from a distribution with the same characteristics as those observed in the material of interest. Moreover, distributions thought to resemble those found in nature, such as Weibull distributions, can be input into the models and the behavior observed.

Statistical models include a variety of lattice, network, molecular-dynamics, cellularautomata, field-theory, and other pseudo-continuum models. Herrmann and Roux (1990) have compiled a comprehensive volume describing statistical models and how they can be used to study fracture in heterogeneous media. They extensively discuss the approximations involved in the various models and the potential for incorporating disorder and scaling results. Meakin $(1991,1992)$ has reviewed several of the lattice models and concludes that while significant improvements are needed, they do reproduce quite well the characteristic crack morphologies observed in a wide range of real systems, and that they provide a solid basis for future development. 


\subsection{Percolation Theory}

One advantage of the statistical models is that they depend on the behavior of discrete sites, and they can be analyzed using percolation theory (Kesten, 1986, 1987; Stauffer, 1985) in association with the concepts of fractal geometry (Peitgen and Sauge, 1988) and self-organized criticality (Bak and Chen, 1989, 1991), to provide a general framework for analysis of the process of fracturing. Using this approach the number and spatial location of cracks and fractures are characterized in a general way. During the fracture process, neighboring grains that are cracked, or otherwise broken, form a cluster, and percolation theory deals with the number and properties of these clusters. A percolating cluster is defined as a group of neighboring cracked grains that - connects two opposite sides of the lattice, and in this type of model a simulated rock is said to fracture when a percolating cluster is formed. Moreover, if there are $N$ sites on the lattice and $n$ of them are cracked, then the concentration of cracked sites, $p$, is given by

$$
p=n / N
$$

We can then define the percolation threshold, $p_{c^{\prime}}$ as the concentration of cracked grains where a percolating cluster is first formed. In percolation theory, $p_{c}$ gives the position of a phase transition where the system changes from an unfractured to a fractured state. This is a critical point in the same sense as a second-order phase transition often associated with gas/fluid systems, and the theoretical machinery developed during the last 30 years to study such phase transitions (e.g., Domb and Green, 1972) can be used to study the behavior of a nucleating or propagating fracture. Several variations of percolation theory have been introduced, including site, bond, invasion (Wilkinson and Willemsen, 1983), and directed-bond (Blease, 1977) percolation, among others. These variations allow the percolation concept to be coupled with a wide variety of numerical models that emulate fundamental physical systems.

Percolation theory provides a powerful approach for developing relations between the statistics of the microstructural heterogeneity and the bulk properties of the rock, such as elastic moduli, and the overall strength as the load or displacement on the rock is increased. This 
approach can be used to describe nonlinear behavior and to develop universal scaling relations that describe the influence of rock sample size on the overall mechanical properties (de Arcangelis, 1990). Moreover, such phenomena as damage, stress corrosion, and rate dependence can be easily incorporated into these models using the concept of annealed disorder (Herrmann and Roux, 1990). Using this concept simple rules are implemented in the model to give the material a memory of its history. For instance, to simulate damage to a grain at each step (in time or stress), grains near an existing crack are damaged by lowering their breaking threshold by an amount proportional to the strain experienced by that grain (Herrmann and Roux, 1990).

Percolation theory can be used to analyze data associated with rock fracture, independent of any statistical model. Chelidze (1986) discussed the relation of percolation theory to acoustic emission observations during rock fracture in the laboratory and to earthquake data and showed how functions used in percolation can be used to interpret these data sets. Sahimi and Arbabi (1992) argued for the existence of universal fixed points for classification of fracture in heterogeneous media such as rock. Rundle and Klein (1993) used percolation theory to analyze scaling properties of clusters of failed sites in simulated faults. Bebbington et al. (1990) reviewed the use of percolation theory for analysis of rock fracture and concluded that although the work of Kesten $(1986,1987)$ has advanced the state of the art, the percolation model is "less attractive" than previously thought. ${ }^{1}$ These authors do find that other types of percolation such as "directed" or "oriented" percolation may provide a better analogy to rock behavior.

\subsection{Application of Statistical Models to Rock Fracture}

To date, much of the work using statistical models has focused on thin films, polymers, concrete, and other engineering materials. Recently, a few of these models have been applied to

1 This is because it is unclear how analysis of experimental data, such as acoustic emissions, relates to real processes in the rock; i.e., acoustic emissions may represent either the energy of the rupture or the area of rupture, and percolation theory has no way to distinguish between the two physical interpretations. 
the study of earthquakes and, to a lesser extent, to the fracture of intact rock. The following paragraphs present a review of the use of statistical models in the study of rock and rock-like materials. While these models incorporate heterogeneity and give discrete realizations of crack geometries and locations, and numbers of broken sites, they have been used mostly to study problems involving tensile stress fields. Few have been applied to rock mechanics problems, as most of these problems involve compressive stresses. The reason for this is that the bond and site elements currently used do not have appropriate behavior for failure in compression.

Conventional bond or site elements do not dilate when failed in compression; rather they collapse and predict behavior that is unrealistic.

\section{Network Models}

Beam models: Herrmann and Roux (1990) present a beam model for fracture that is a straightforward discretization of the Cosserat equations for elasticity, and Schlangen (1993) and Schlangen and van Mier (1992) have adapted this model for analysis of fracture in concrete. These authors introduced heterogeneity into the simulated material in three ways: by assigning random strength values to beams on a regular lattice, by generating a random particle structure and assigning different strength values to the beam elements in the various composite materials (spatially correlated strength values), and by assigning constant strength values to beam elements in a random lattice. They found the model to be useful in prediction of fracture of concrete and to produce such phenomena as crack face bridging and curved crack growth. In this model, a scaling factor is used to fit strengths predicted by the model to those observed in experiments.

Molecular dynamics: Mora (1992) used an atomic-lattice approach based on molecular dynamics to study earthquakes and the development of fault systems, and produced a complex system of fractures that have some similarity to tectonic fault systems. Huang (1992) and Huang and Kim (1993) used a network of particles and springs under conditions of dynamic loading to simulate hydrofracture at a borehole, and found that the fracture pattern depends on the heterogeneity, the active crack-driving force, and the in situ stresses. However, the pattern of cracks produced in these simulations appeared to be controlled by the triangular lattice used in 
the simulation. More recently, Song and Kim (1994) used the same model to simulate delay blasting in mining and quarrying.

\section{Cellular Automata}

Rundle and Klein (1993) have studied earthquakes recurrence relations and scaling relations using an automaton as mentioned above, and an automaton model was also used by Steacy and Sammis (1991) to simulate fragmentation in fault zones. These authors found that the automaton model produced crack patterns and particles sizes with the same fractal dimension as those observed in fault zones.

\section{Thermodynamic Model}

Englman and Jaeger (1990) modeled damage in concrete by reformulating crack growth in terms of a crack population in a porous medium. The porous material was simulated in three dimensions using a site-bond percolation system and the number density of microcracks was used as the independent variable. Percolation theory was then used to express strain energy as a function of crack density, and thermodynamic and energy minimization criteria were applied to assess the properties of the concrete.

\section{Diffusion Limited Aggregation (DLA) Models}

The diffusion limited aggregation model has been used to simulate fracture by developing an algorithm that adds cracked sites one at a time to a growing cluster of cracked sites in a simulated material (Meakin, 1991). In this type of model an initial crack is inserted in a region of interest and a stress or displacement boudary condition is applied to the region. A rule is developed for computing the probability of cracking any site on the perimeter of the crack. The rule may be based on the amount of deformation or stress in the site, or on another criterion. This type of model has been used with mixed success to simulate tensile fracture in heterogeneous materials (Louis and Guinea, 1985; Hinrichsen et al., 1989). 


\subsection{Nearest-Neighbor Model}

\subsection{Modeling Description}

In this chapter a unique, nearest-neighbor model for rock fracture is presented. This model is based on the premise that at the grain scale each grain is acted on by forces at the grain boundary, and consequently the behavior of each grain depends on the stresses transmitted through its neighbors. Simulating rock fracture on the grain scale can be approached deterministically by simulating grain shapes and strengths and grain contacts in a detailed way. This is an active area of study, and Liu et al. (1993), Bruno and Nelson (1991), and others have proposed models of this sort. As discussed previously, the purpose of this thesis is to develop methods that incorporate disorder in a stochastic way, rather than a discrete way as is done in the models discussed above. An alternative approach is to simplify the problem of site geometry and make all particles the same shape (say, circular in two dimensions) and represent the complicated site geometries and associated stress variations using a local site shape factor. Another simplification is to assume that all grains are perfectly bonded and that all cracks form by grain breaking. These assumptions are the basis of the nearest-neighbor model developed in this section. In this model a breaking rule based on fracture mechanics is used, along with the concepts of percolation to describe fracture.

This model simulates the rock in two dimensions with an array of disks of diameter $D$, arranged on a square lattice. The disks are assumed to be rigidly bonded at their points of contact, and uniform stress conditions in the form of axial $\left(\sigma_{\mathrm{I}}\right)$ and confining $\left(\sigma_{\mathrm{II}}\right)$ stresses are imposed on opposite sides of the array. Initially, all disks are intact and stresses are uniformly distributed (Figure 3.1-1 a). Boundary stresses can be changed arbitrarily and at each stress condition a breaking rule (described in Section 3.1.1) is evaluated and the disk with the highest potential (above a threshold) is allowed to crack (break). The disks are cracked one at a time, and after each cracking event the stresses in the neighborhood of the cracked disk are redistributed based on a nearest-neighbor rule (described in Section 3.1.2). This cycle of cracking and stress 


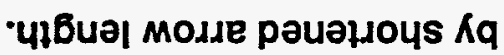

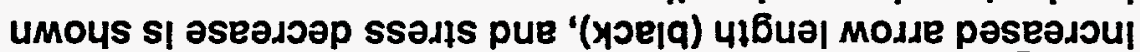

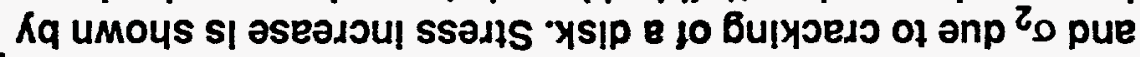

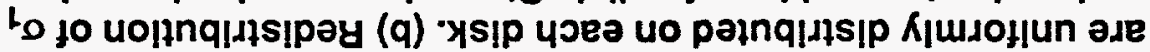

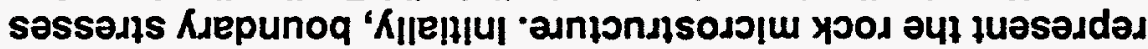

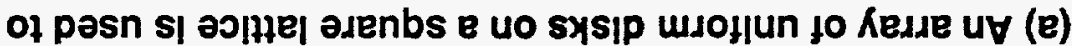

$\because-レ \cdot \varepsilon$ วגn6!」
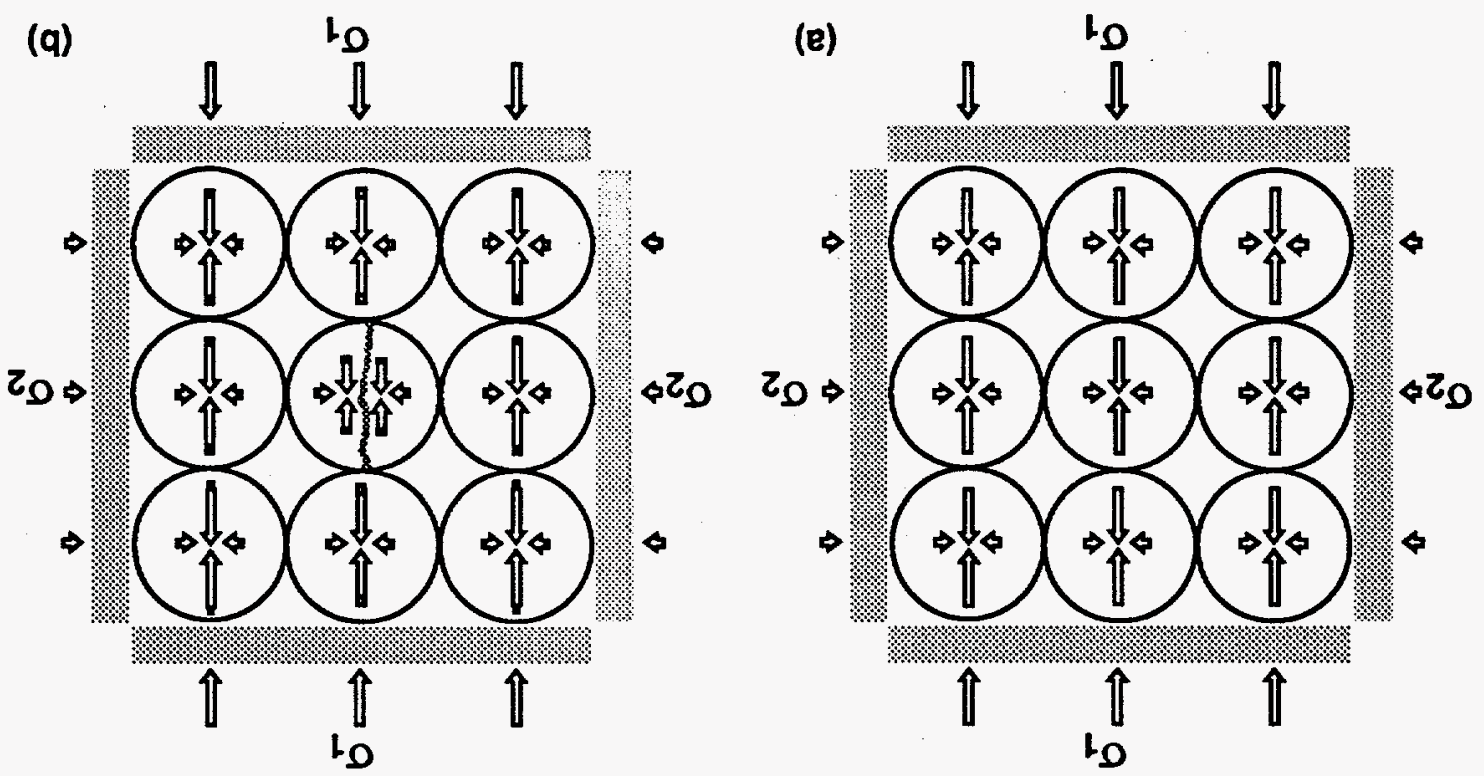
redistribution continues until no other cracking events occur at the given stress level. Each time a disk cracks, the array is searched and clusters of cracked disks are identified. Rock fracture is defined as occurring when one cluster spans the lattice. The model was used to simulate uniaxial and triaxial compression tests on rock with heterogeneous grain strength distributions. The following subsections describe the model in more detail.

The basic form of the model is shown in Figure 3.1-1. The rock is simulated in two dimensions as an array of uniform disks.

\subsubsection{B reaking Rule}

The breaking rule for this model is based on fracture-mechanics analysis presented by Kemeny and Cook (1991). This rule is formulated as follows: each disk is assumed to contain a microcrack and at a given stress condition the stress-intensity factor $\left(K_{\mathrm{I}}\right)$ for the microcrack in each disk is computed as:

$$
K_{\mathrm{I}}=\frac{2 c_{1}\left(\sigma_{1}-c_{2} \sigma_{2}\right) \sqrt{l}}{\sqrt{\pi}}\left[\left(2-\frac{l}{\alpha}\right) \sin ^{-1}\left(\frac{\alpha}{l}\right)+\sqrt{1-\frac{\alpha^{2}}{l^{2}}}\right]-\sigma_{2} \sqrt{\pi},
$$

$$
\text { where } \begin{aligned}
c_{1} & =\text { constant } \\
c_{2} & =\text { stochastically assigned stress concentration factor } \\
\sigma_{1} & =\text { maximum principal stress (axial) } \\
\sigma_{2} & =\text { minimum principal stress (confining) } \\
l & =\text { crack length }=\text { disk diameter } \\
\alpha & =\text { constant }
\end{aligned}
$$

following Kemeny and Cook (1991). This representation of $K_{\mathrm{I}}$ incorporates a modified Coulomb criterion for failure in the form of the $\left(\sigma_{1}-c_{2} \sigma_{2}\right)$ term. The disk may crack (break) if $K_{\mathrm{I}} \geq K_{\mathrm{IC}}$ when the length of the microcrack equals the disk diameter, where $K_{\mathrm{IC}}$ is the critical stressintensity factor. This rule is evaluated for each disk at each stress condition. 


\subsubsection{Stress Redistribution}

In this model, it is assumed that when a disk cracks it can support only a reduced level of axial stress, and that there is a crack opening displacement (COD) associated with the crack that will cause a local increase in confining stress along the sides. Further, stress no longer carried by the cracked disk is redistributed to disks that are nearest neighbors to the cracked disk. A disk is a nearest neighbor if it borders the cracked disk. This includes the side, top, bottom, or diagonal directions. In the $\sigma_{1}$ direction, it is assumed that

$$
\sigma_{1}(\text { cracked })=a \sigma_{1} \text { (intact) },
$$

where $a<1$. To compensate for the local reduction in stress, $\sigma_{1}$ is increased on the disks on either side of the cracked disk, so that $\sigma_{1}$ is balanced across any plane perpendicular to the direction of $\sigma_{1}$. The stresses on the neighboring disks above and below are assumed to support a reduced level of $\sigma_{1}$ as well $\left(\sigma_{1}\right.$ (top) $=\sigma_{1}$ (bottom) $=\sigma_{1}$ (cracked) $)$ and, to compensate, $\sigma_{1}$ is increased on the disks diagonal to the cracked disk. The stress redistribution is illustrated in Figure 3.1-1 b.

In the $\sigma_{2}$ direction, the COD causes an increase in $\sigma_{2}$ in each half of the cracked disk and in the disks on either side. Sammis and Ashby (1986), along with other workers, have shown that along a crack parallel to the $\sigma_{1}$ direction, $\sigma_{2}$ is a function of the length of the crack and the applied $\sigma_{1}$. The increase in $\sigma_{2}$ is computed as

$$
\sigma_{2}(\text { cracked })=b\left(\sigma_{1}\right)+\sigma_{2}
$$

where $0<b<<1$. In addition, it is assumed $\left[\sigma_{2}\right.$ (side) $=\sigma_{2}$ (cracked)]. To compensate for the local inverse in $\sigma_{2}, \sigma_{2}$ is reduced in the disks above, below, and diagonal to the cracked disk. The redistribution of $\sigma_{1}$ and $\sigma_{2}$ due to cracking of a disk is illustrated in Figures 3.1-1 a and b. If a cracked disk occurs on a boundary, stresses on the neighboring disks are computed assuming a reflective boundary geometry.

\subsubsection{Heterogeneity}

The natural variation in rock mineralogy, and in the size, shape, and loading geometry of individual grains, affects the strength of individual grains as well as the local differential stress 
that is applied on them (Zheng 1989). However, as is shown in Figure 3.1-1, in the nearestneighbor model all the disks are the same size and all have the same loading geometry.

Heterogeneity of strengths and loading geometries found at the grain scale in rocks is simulated by assigning each disk a value of $c_{2}$ picked from a random distribution of values between 2 and 8 . These values are consistent with values used in fracture mechanics analysis. Thus, the differential stress computed for the $j$-th disk is:

$$
\sigma_{1}(j)-c_{2}(j) \sigma_{2}(j)
$$

where the algorithms for calculation of $\sigma_{1}(j)$ and $\sigma_{2}(j)$ are described above.

\subsubsection{Estimation of Axial Strain}

It is assumed that both intact and cracked disks behave elastically, and that Young's modulus $(\mathrm{E})$ is lower for the cracked disks than for the intact disks. Young's modulus for the cracked disks is assumed to be

$$
E_{\text {cracked }}=\frac{E_{\text {intact }}}{2} \text {. }
$$

As stated above, it is also assumed that a cracked disk supports a reduced level of axial stress.

Thus at any given level of axial stress, the strain for intact and cracked disks can be found as shown in Figure 3.1-2. Total axial strain $(\varepsilon)$ is then computed as a simple summation:

$$
\varepsilon_{\text {total }}=\varepsilon_{\text {cracked }}+\varepsilon_{\text {intact }}
$$

where $\varepsilon_{\text {cracked }}$ and $\varepsilon_{\text {intact }}$ are given by

$$
\varepsilon_{\text {cracked }}=\sum_{i=1}^{\# \text { cracked }} \frac{\sigma_{1}(i)_{\text {cracked }}}{E_{\text {cracked }}}
$$

and

$$
\varepsilon_{\text {intact }}=\sum_{j=1}^{\# \text { intact }} \frac{\sigma_{1}(j)_{\text {intact }}}{E_{\text {intact }}} .
$$




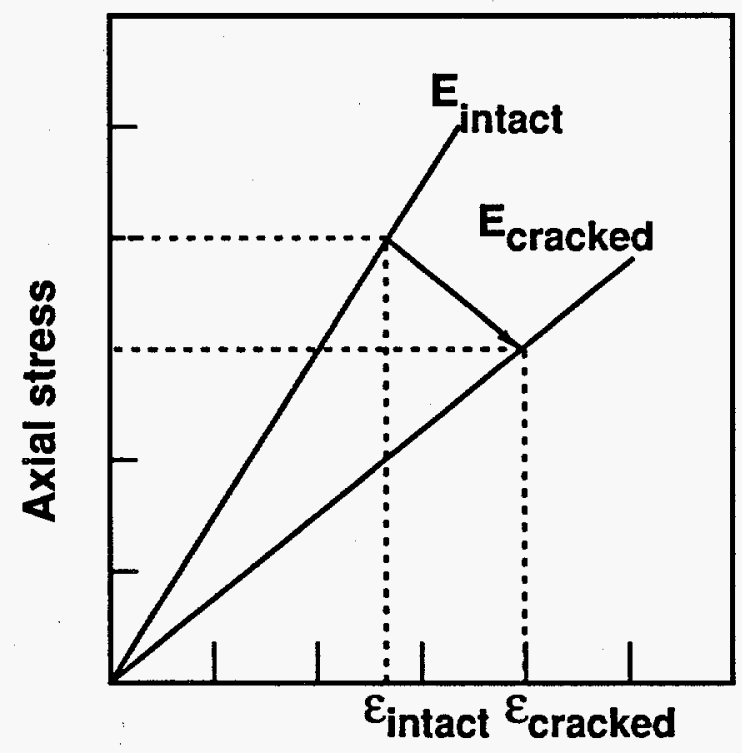

Axial strain

Figure 3.1-2. Stress-strain behavior for Intact and cracked disks. Cracked disks can support only a reduced amount of axlal load. 


\subsubsection{Crack Coalescence and Rock Fracture}

The nearest-neighbor model simulates the coalescence of microcracks into cracks, stable crack growth at a constant level of compressive stress, and the eventual fracture via a transverse crack. The computation logic is illustrated in Figure 3.1-3. First the model is initialized, random values of $c_{2}$ are assigned to disks, and initial stresses are applied. $K_{\mathrm{I}}$ is calculated for each disk and the disk with the highest value is identified. If $\mathrm{K}_{\mathrm{I}}(\max ) \geq \mathrm{K}_{\mathrm{IC}}$, this disk is cracked and the stresses are redistributed. The values of $K_{\mathrm{I}}$ for all uncracked disks are recomputed and again $K_{\mathrm{I}}(\max )$ for all uncracked disks is identified. If $K_{\mathrm{I}}(\max ) \geq K_{\mathrm{IC}}$, this disk is cracked and stresses are recomputed, with the process continuing until no more disks crack at the given stress level. Each time a cracking event occurs the array is searched and clusters of cracked disks are identified. Cracked disks belong to the same cluster if they are nearest neighbors. Fracture is defined as follows: the perimeter of the array is divided into six segments (A-F) as shown in Figure 3.1-3. Fracture occurs when one cluster of cracked disks makes one of the following connections; $A-C$, A-D, A-E, B-D , B-E, D-F, C-F. This rule is discussed in more detail in Chapter 4. An example of a cluster causing fracture by connecting segments $B$ and $E$ is shown in Figure 3.1-3. The logic of the numerical code used to implement this model is shown in Figure 3.1-4.

\subsection{Results-Simulation of Compression Tests}

Stress-strain behavior for a 15 by 30 array of disks was calculated using the nearest-neighbor model by simulating standard triaxial tests at three different confining stresses $(10,20$, and $40 \mathrm{MPa}$ ). In these calculations, $\mathrm{E}_{\text {intact }}=60 \mathrm{MPa}, K_{\mathrm{IC}}=1 \mathrm{MPa} / \mathrm{m}^{1 / 2}, a=0.25$, and $b=0.1$. Uniaxial and triaxial laboratory compression tests were simulated as step loading tests. Confining stress, $\sigma_{2}$, was applied to all boundaries; then axial load was incrementally increased.

The stress-strain curves for these simulations are shown in Figure 3.2-1. This figure shows that the model predicts nonlinear stress-strain behavior similar to that observed in laboratory tests. The behavior includes an increase in strength with increasing confining stress and initial strain hardening as increases in stress produce increased strain. The spatial distribution of 


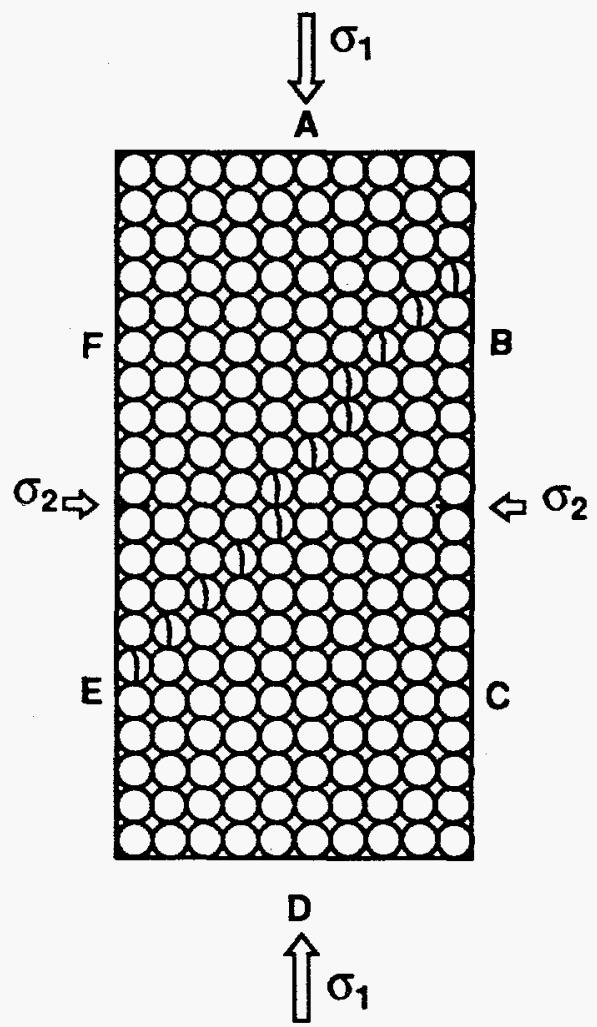

Figure 3.1-3. Fracture is defined as occuring when a cluster of cracked disks spans the array. The perimeter above is divided into segments (A-F), and the array shows a fracture is formed when a cluster of cracked disks connects segments $B$ and $E$. 


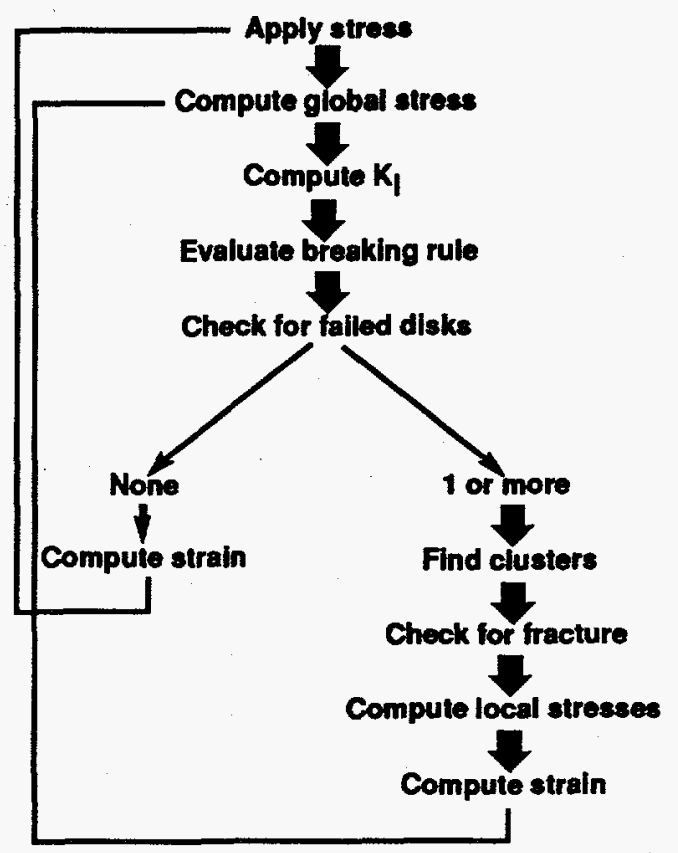

Figure 3.1-4. Loglc of calculation. 


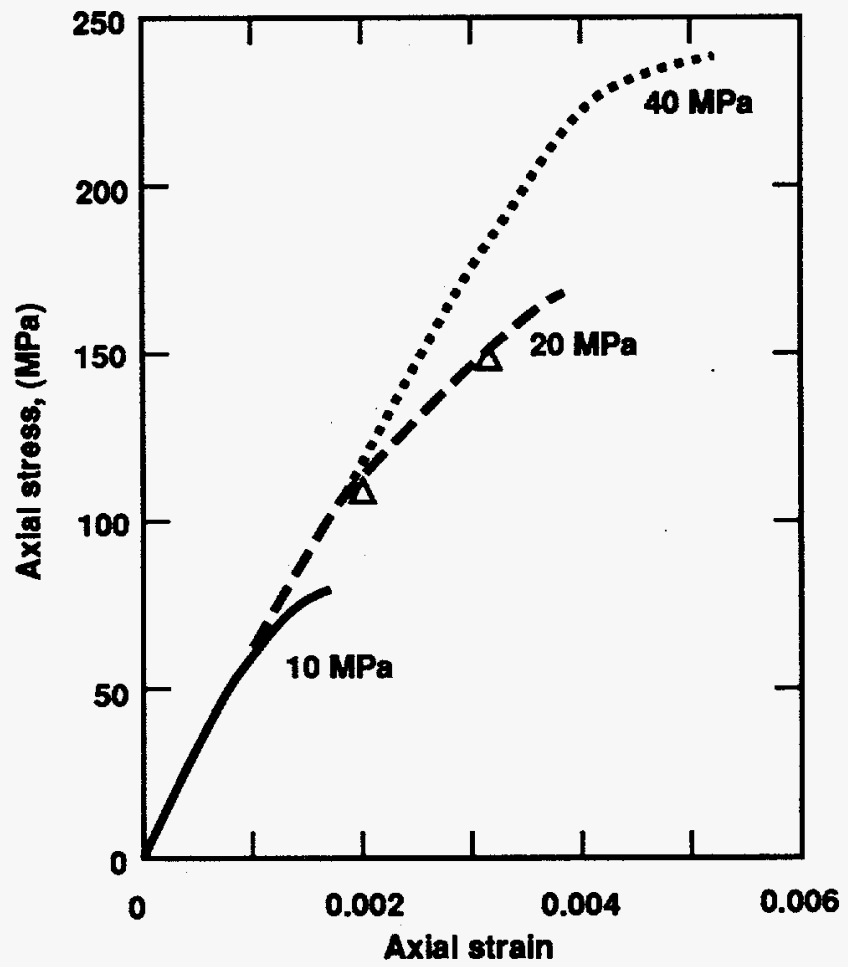

Figure 3.2-1. Stress-strain curves predicted for a 15 by 30 array at confining stresses of 10,20 , and $40 \mathrm{MPa}$. 
cracked disks is imaged for two points on the curve for the trial with confining pressure of $20 \mathrm{MPa}$. These points are denoted by triangles on Figure 3.2-1. These images are shown in Figures 3.2-2 $a$ and $b$. In these images, the uncracked disks form the black background and the cracked disks are denoted by grey or white square pixels. The brightness of a cluster of pixels is keyed to the number of pixels in the cluster, with largest clusters being brightest. Figure 3.2-2 a shows the spatial distribution of cracked disks at an axial stress of $110 \mathrm{MPa}$. Two main clusters are visible in this image; the largest is in the upper left region, and a somewhat smaller cluster is shown in the center of the image. Figure $3.2-2 \mathrm{~b}$ shows the spatial distribution of cracked disks at an axial stress of $150 \mathrm{MPA}$. Several disks in the upper right of the image have cracked, and a large cluster has formed that includes these disks along with the center cluster from the previous image. A large cluster has also formed in the lower left region, and these two major clusters dominate the image. The cluster in the upper left has increased in size, but it is no longer dominant, which shows that the first cluster to form does not necessarily continue to dominate the array.

These results show that the nearest-neighbor model allows clusters of cracked disks to extend and/or coalesce to form fractures and predicts stress-strain behavior similar to that observed in rocks. The results of this model are promising; moreover, the model is very fast numerically and has potential application for the fracture of discrete grains, for example, in communition problems. However, this model does not readily handle large problems or excavations, and further development and application is left for future work. This approach may prove to be particularly suited to analysis of fracture of clastic rocks because the grains are often discrete and somewhat circular. 

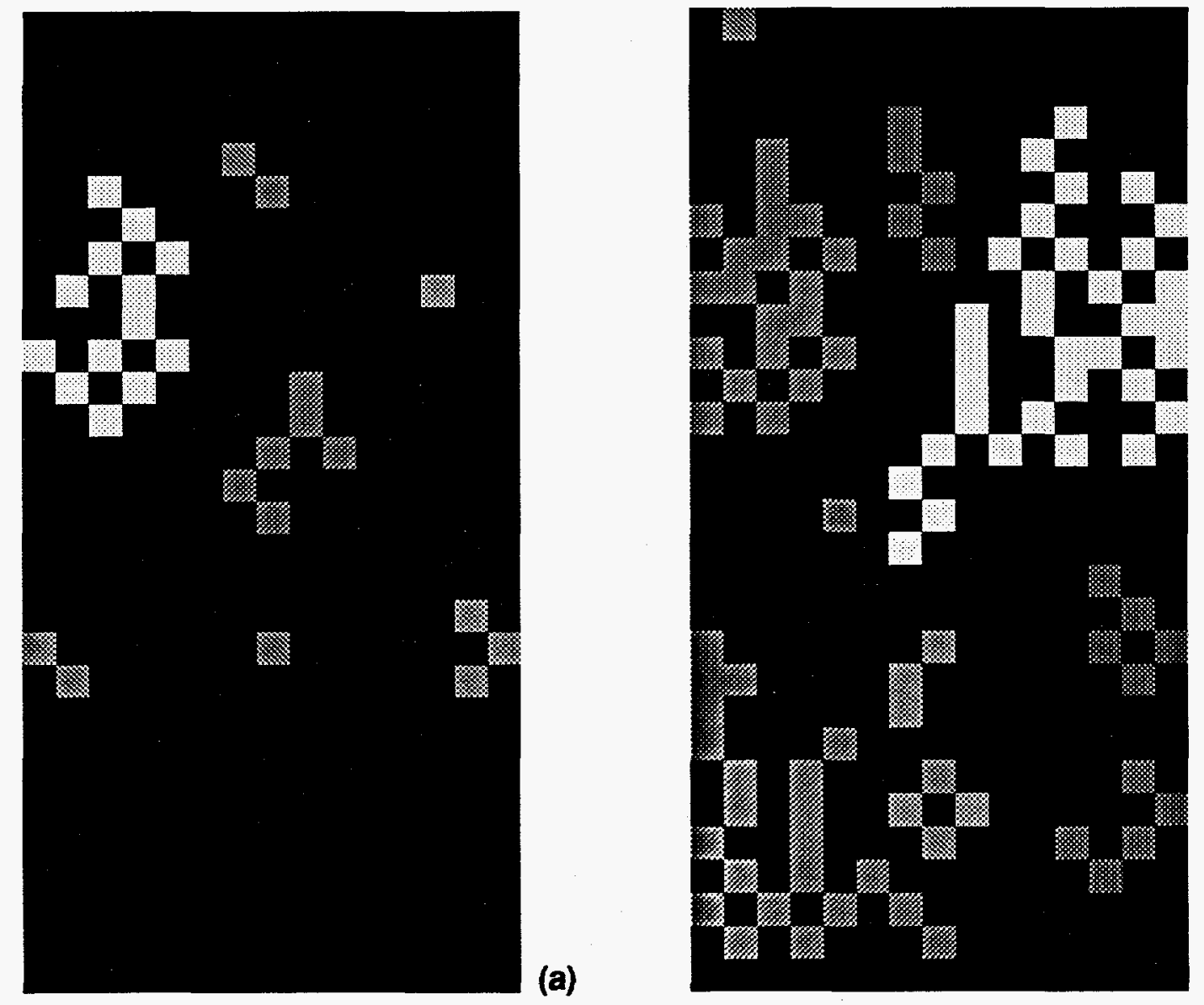

(b)

Figure 3.2-2. Locations of cracked disks for 2 points on the $20 \mathrm{MPa}$ confining stress curve shown in Figure 3.2-1: (a) locations of cracked disks at axial stress of $110 \mathrm{MPa}$; (b) locations of cracked disks at $150 \mathrm{MPa}$. See text for discussion. 


\subsection{Field-Theory Model}

This chapter presents a numerical model for fracture of rock in compression that is based on a field-theory approach to the analysis of microcracking at the grain scale. This approach incorporates the concept of superposition, and the techniques of boundary element analysis (Crouch and Starfield, 1990) and percolation theory (Stauffer, 1985), to form a simple yet powerful method for studying progressive fracture of rock and other heterogeneous materials.

The model has been designed to incorporate many of the aspects of rock fracture learned from the experiments and analyses discussed in Chapter 2. These include fracture (in compression) via the formation, extension, and coalescence of extensile cracks that are generally oriented in the direction of the maximum principal stress; heterogeneity in the microstructure due to variation in strength of grains; and creation at the microscale of local tensions that may be caused by heterogeneity in the shape, orientation, or loading of grains. This model also can incorporate heterogeneity in the strength of grains and has the ability to incorporate load path dependence into the local stress and strain fields so that when a crack forms and opens, some strain energy is stored in the rock and some strain energy is dissipated and is not recovered upon unloading.

In this chapter the principles of the model are presented (Section 4.1) and the implementation of the model using the boundary element method (BEM) is discussed in detail (Section 4.2). Section 4.3 describes the lattices used and Section 4.4 shows how the model was configured to simulate standard laboratory compression tests to produce macroscopic stress-strain behavior. An overview of the numerical codes comprising the model is presented in Section 4.5. 


\subsection{Principles}

\subsubsection{Overview of Concepts Used in Field-Theory Model}

\section{Treatment of Cracks}

The literature review presented in Chapter 2 shows that in compression rocks deform and fracture by the formation, extension, and coalescence of extensile opening mode cracks. Moreover, Jaeger and Cook (1976) have shown that when cracks form in a material, part of the energy of loading is dissipated in the material and is not recovered upon unloading. This energy may be dissipated by inelastic deformation of the region around crack tips. This concept is illustrated in Figures 4.1-1 a-d. Consider a material with an incipient crack, under a tensile load $F$ due to the weight of a block. The material response is linear elastic when a load $F$ is applied, as shown in Figure 4.1-1 a, and the stress-strain response is represented by segment $\mathrm{O}-\mathrm{A}$ in Figure 4.1-1 b. The trace of an incipient crack in the material is shown in the material in Figure 4.1-1 a. It is assumed that the strength of the material is such that when a small increment of load, $\Delta F$, is applied, the crack forms and opens as is shown in Figure 4.1-1 c, and the displacement (strain) increases at constant load $F_{1}=F+\Delta F$ (stress $\sigma_{1}=\sigma+\Delta \sigma$ ). This increase in strain is shown as increment A-B on the stress-strain curve in Figure 4.1-1 d. When the tensile stress is removed from the cracked material, the stress-strain curve moves along segment $\mathrm{B}-\mathrm{O}$. Then the energy recovered upon unloading can be represented by area $\mathrm{O}-\mathrm{B}-\mathrm{C}$. Using the formula for the area of a triangle, area $=(1 / 2) b h$, the relations between different segments of the stress-strain curve can be written as

$$
\text { Area }(\mathrm{OBC})=(1 / 2)(\mathrm{BC})(\mathrm{OD}+\mathrm{DC})
$$

but $\mathrm{DC}=\mathrm{AB}$, so that

$$
\begin{aligned}
\text { Area }(\mathrm{OBC}) & =(1 / 2)(\mathrm{BC})(\mathrm{OD})+(1 / 2)(\mathrm{BC})(\mathrm{AB}) \\
& =\text { Area }(\mathrm{OAD})+\text { Area }(\mathrm{OAB}) \\
& =\text { Intrinsic }+ \text { crack opening. }
\end{aligned}
$$




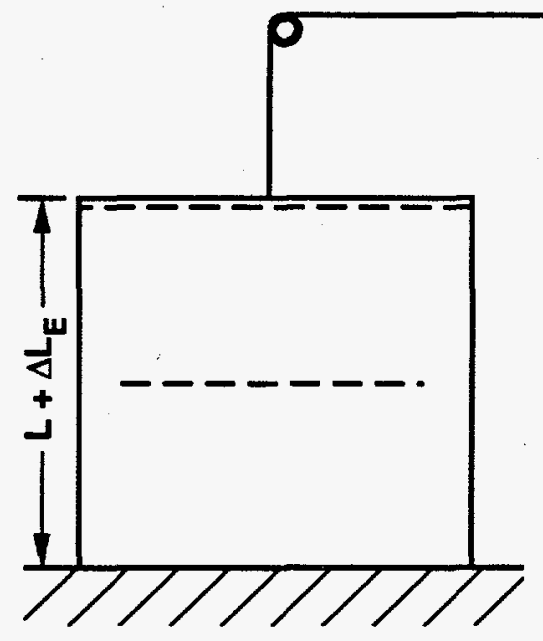

a)

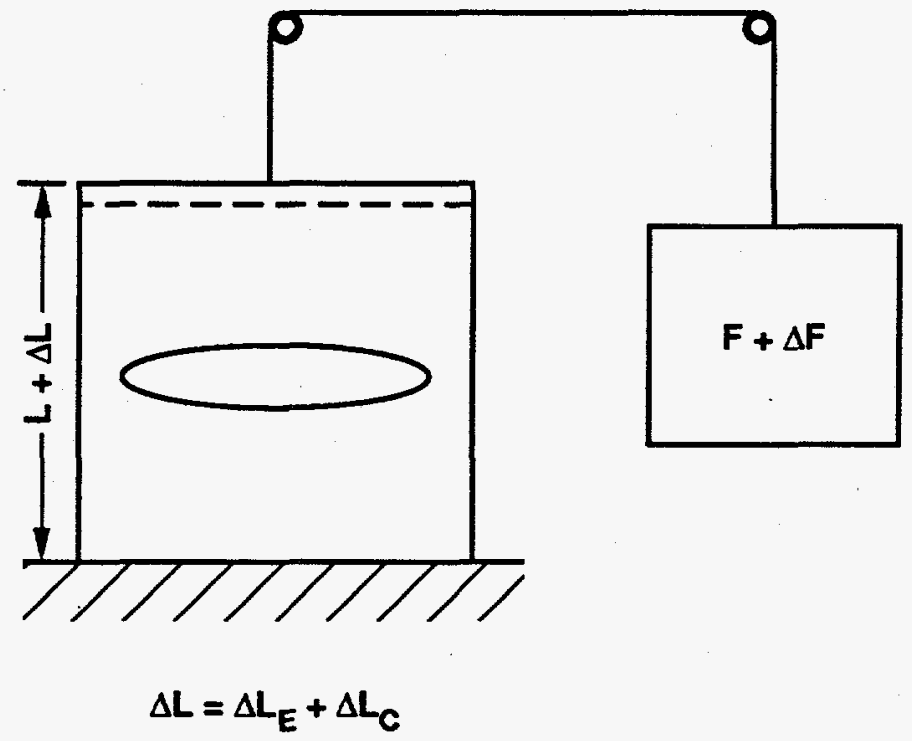

c)

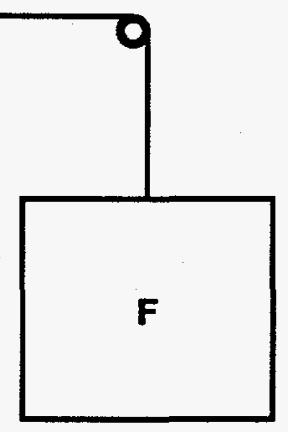

$\sigma$

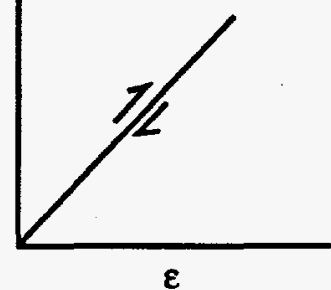

b)

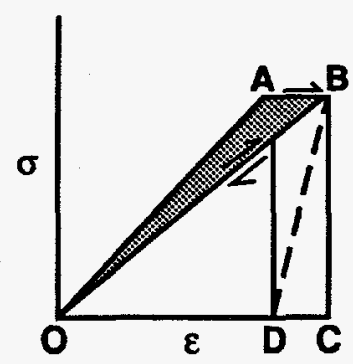

d)

Figure 4.1-1. Stress strain behavior assoclated with opening of a crack. Figures $a-d$ are discussed in the text. 
This shows that the effective stress-strain behavior and stored strain energy of the cracked material (given by the unloading and reloading curve $\mathrm{OB}$ ) is composed of a component determined by the intrinsic or matrix properties of the material, and a component associated with the crack opening in the material.

Moreover, the total work done on the material by the opening of the crack is given by

$$
\mathrm{W}=\text { Force } \times \text { distance }=\text { Area }(\mathrm{ABCD}) \text {. }
$$

Now the additional strain energy recovered after opening the crack is

$$
\text { Area }(O A B)=(1 / 2) \text { Area }(A B C D) \text {. }
$$

Thus one half of the work done on the material is recovered as strain energy; the remaining half is dissipated as inelastic deformation. Upon reloading, the cracked material will respond along segment $\mathrm{O}-\mathrm{B}$. This simple analysis shows that when cracks open in a material, strain energy is dissipated; this energy is dependent on the stress at which cracks form and on the load path, and is equal to the strain energy due to the opening of cracks. Note that strain energy is dissipated during crack formation, but the cracked body acually has more strain energy than the uncracked body.

Many models for rock behavior can incorporate cracks and fractures, but often when cracks are introduced, the unrecoverable work done on the material due to the cracking process is lost. The field-theory model presented in this chapter attempts to capture this aspect of material behavior. The model is based on the assumption that the stress field in a cracked rock can be decomposed into those of an elastic continuum and a cracked elastic body. This assumption is formulated using the concepts of superposition and crack tractions. By using linear systems the uniqueness of any solution is guaranteed.

The fundamental concept of crack formation used in this model is presented in Figure 4.1-2, which poses the problem discussed in Figure 4.1-1 in terms of superposition and crack tractions. Figure 4.1-2 a shows a body $R$ with an incipient crack loaded by a force $F$. An incipient crack has been placed in the material and the crack is held closed by crack-closing tractions (cct's). This figure also shows the decomposition of the stress field in $R$ into stress field $S$ in (1) an uncracked 

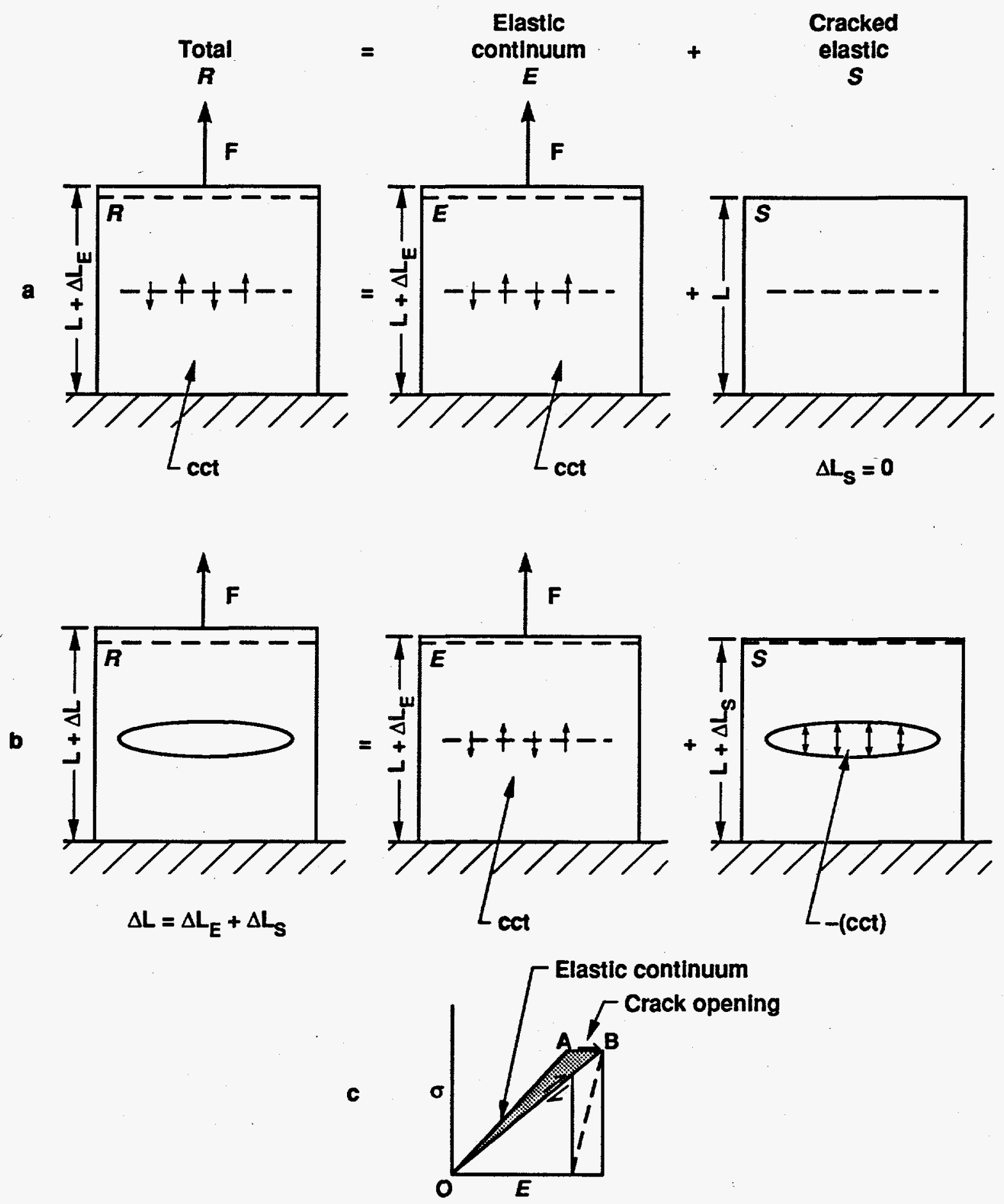

Figure 4.1-2. Decomposition of stress field into elastic continuum and cracked elastic body: a) stress decomposition of body with an inciplent crack, b) stress decomposition used to form crack in $R$. c) stress strain response incorporating crack opening. 
elastic continuum, $E$, and (2) a cracked elastic body, $S$. When the crack is closed the crack-closing tractions appear in $E$, the elastic continuum, but not in $S$, the cracked body. The crack-closing tractions can be thought of as the stresses in a very thin layer of glue that is holding the crack closed (of course the glue has the same material properties as the material in $E$ so that the crack is not detectable). The stress-strain curve for Figure 4.1-2 $\mathrm{a}$ is shown as segment $\mathrm{O}-\mathrm{A}$ in Figure 4.1-2 c.

If a small increment of force, $\Delta F$, is applied to the body so that the glue breaks and the crack is allowed to open, then the behavior is that shown in Figure 4.1-2 b. This figure shows how the principle of superposition is used to simulate the release of the crack-closing tractions (cct's). The crack in $R$ is formed by first opening the crack in $S$ by imposing crack-opening tractions (the opposite of crack-closing tractions) on its internal surface, and then superposing the stress fields in $E$ and $S$. The crack-opening tractions in $S$ have the same magnitude as, but opposite direction to, the crack-closing tractions in $E$. Thus, superposition of the stress fields in $E$ and $S$ forms the stress-free surface of an open crack in $R$. The stress-strain behavior of this system is illustrated in Figure 4.1-2 c, which shows that this method also allows dissipation of energy associated with crack formation.

This simple case shows how the stress field in a material in which cracks form can be decomposed into two components, representing stress in an uncracked elastic continuum and in a cracked elastic body, which are then recombined using superposition. This technique provides a convenient method for studying the stresses due to cracks in a material.

\section{Treatment of Heterogeneity}

Another important feature of the field-theory model is the incorporation of heterogeneity. As discussed in Chapter 2, continuum models can easily handle compressive stresses but do not readily include heterogeneity, while statistical, lattice-based models can incorporate heterogeneity but they do not adequately simulate fracture of rock in compression and are not well equipped to handle complex boundary conditions. In the field-theory model, the concept of a lattice is used in conjunction with the elastic continuum approximation to simulate crack 
formation and extension under conditions of compressive stress. This is done by first describing the general problem to be analyzed with a continuum approximation, and then overlaying a lattice on areas of interest where cracking is expected in the elastic continuum problem. The lattice serves several purposes. One of the most important is that it is used as a vehicle for incorporating local heterogeneity. For instance, in this study the sites are taken to represent the centroids of grains that compose a rock. Moreover, individual strengths and geometric parameters are assigned to each site in order to simulate the heterogeneity in composition, and shape and loading geometry of grains that form the rock matrix. In addition, cracks are only allowed to form at sites on the lattice. This allows the introduction of a breaking rule, which can be easily modified to address different fundamental assumptions about the material behavior, thereby providing the model with a great deal of flexibility. The use of a lattice also provides a mechanism for accessing percolation theory. This provides a way to formulate a failure criterion and to evaluate scaling relations. In addition, use of a lattice simplifies the problem because it limits the area for detailed solution to a local region of interest (Schlangen, 1993).

The concept of stress decomposition and use of a lattice to incorporate heterogeneity have been used to formulate a field-theory model for progressive fracture of rock in compression. The remainder of this chapter shows how these concepts were implemented into a numerical code to study rock behavior in compression.

\section{Overview of Implementation}

As discussed above, the field-theory model is based on the assumption that the total stress field in a rock or other heterogeneous material can be decomposed into an elastic continuum component and a crack interaction component, which are then recombined using superposition. The elastic continuum approximation provides an estimate of the stress and deformation fields in a region of interest. Areas of high stress, where cracking or fracture are expected, are identified and a lattice of sites is overlaid, which provides discrete sites for cracking to occur. The focus of this study is on the effect of microstructural heterogeneity at the grain scale, and the lattice sites are used to represent the centroids of grains forming a rock matrix. Stresses and displacements 
are evaluated at each of these sites. Heterogeneity in rock microstructure due to variations in the strength and shape of grains forming a rock matrix is simulated by associating a tensile strength and a stress perturbation with each site on the lattice. The stress perturbation can be either compressive or tensile and provides a means of simulating local tension in a grain even in a compressive stress field. The superposition analysis of crack opening given above is extended to simulate formation of cracks in a material under compression by incorporating the stress perturbation term. A simple breaking rule is defined as follows: if the stress at a site is tensile and exceeds the tensile strength assigned to the site, that site is said to crack or "break." If tensile stress exceeds the tensile strength at more than one site, the site with the most overstress is cracked. Clusters of cracked sites are identified and the sample is said to fail when a percolating cluster is formed. A percolating cluster is a group of neighboring cracked sites that span the lattice in a prescribed way. The stress field is recalculated after each breaking event and the breaking rule is re-evaluated for the sites that are still unbroken. At a given stress state, additional sites are broken until (a) no more sites break, or (b) a percolating cluster is formed. The following sections describe the details of the field-theory model and how it is implemented.

\subsubsection{Analysis of Stress}

First, consider an area $R$ imbedded in a body or region of interest. This body or region can be of arbitrary shape, may contain fractures and openings, and may be subject to a variety of boundary conditions. In this analysis it is assumed that the stress at site $i$ in $R$ can be decomposed into components appropriate to an elastic continuum $E$ and a cracked elastic solid $S$ with shape and excavations the same as $R$. This decomposition is illustrated in Figure 4.1-3. Area $E$ is imbedded in an uncracked elastic continuum with boundary or far-field stresses the same as

$R$. Area $S$ is embedded in a cracked elastic material, with zero stress on the boundary.

$R$ is chosen to be in a region where crack formation or extension is expected, for instance, near an underground opening (see Figure 4.1-3), and a lattice of sites is overlaid on $R$. Each site on the lattice is taken to represent the centroid of a grain in the rock comprising $R$. Equivalent lattices are overlaid on areas $E$ and $S$. The general formula for stress in $R$ is given by 


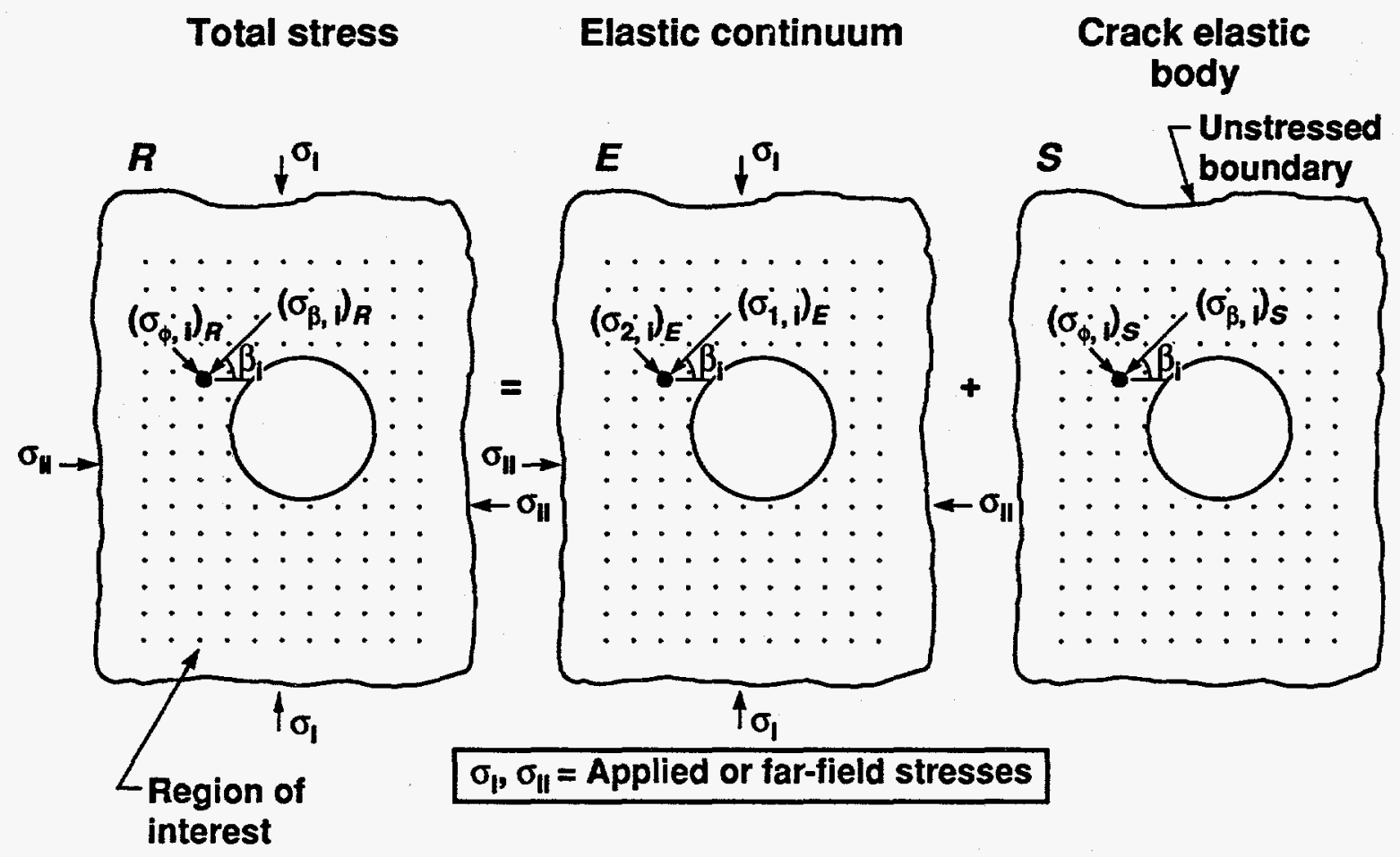

Figure 4.1-3. Stress field decomposition at site I used in the field-theory model. 


$$
\sigma_{R}=\sigma_{E}+\sigma_{S}
$$

The local stress at any site $i$ on the lattice in $R$ can be written in terms of stresses at site $i$ in $E$ and $S$. In particular, the stress in $R$ at a site $i$ in direction $\theta$ can be written as

$$
\left(\sigma_{\theta, i}\right)_{R}=\left(\sigma_{\theta, i}\right)_{E}+\left(\sigma_{\theta, i}\right)_{S}
$$

Now at each site $i$ a local maximum and minimum principal stress can be computed for each array. In particular, the magnitudes and directions of the local maximum and minimum

principal stresses in the continuum array $E$ are given by $\left(\sigma_{1, i}\right)_{E^{\prime}}\left(\sigma_{2, i}\right)_{E^{\prime}}\left(\beta_{i}\right)$, and $\left(\phi_{i}\right)$, respectively. Following equation 4.2 we have

$$
\begin{aligned}
& \left(\sigma_{1, i}\right)_{R}=\left(\sigma_{1, i}\right)_{E}+\left(\sigma_{1, i}\right)_{S} . \\
& \left(\sigma_{2, i}\right)_{R}=\left(\sigma_{2, i}\right)_{E}+\left(\sigma_{2, i}\right)_{S} .
\end{aligned}
$$

Area $E$ is used to determine those aspects of the stress field associated with purely elastic continuum response of the rock in $R$. Area $S$ is used to determine aspects of the local stress field associated with crack formation, opening, and interaction.

\subsubsection{Introduction of Microscale Heterogeneity}

\section{Site-Strength Heterogeneity}

Rocks are known to have microscale heterogeneity in strength due to local variations in mineralogy and cementation of grains, degree of weathering and alteration of the matrix, and other factors. Microscale heterogeneity in strength is incorporated in the field-theory model by assigning a tensile strength, $T_{g^{\prime}}$ to each site. The strength value, $T_{g, i}$ for each site $i$ is a stochastic variable and is selected from a prescribed distribution of strength values. These values may or may not have spatial correlation, and in this study both uniform and bimodal distributions with no spatial correlation are used. The site tensile strengths are assigned at the beginning of a trial and do not change. In the terminology of statistical models, this is known as quenched heterogeneity in site strength. 


\section{Geometric Heterogeneity at Sites}

The microgeometry of rocks is also known to be heterogeneous. In particular, variations in the shape and loading geometry of the grains can cause local areas of tension, even in a compressive stress field. This is illustrated in Figure 4.1-4. Microscale heterogeneity in the stress field due to stress concentrations within grains is simulated in the field-theory model by introducing a perturbation to the continuum stress field. This is done by assigning a stress perturbation factor, $\rho$, to each lattice site in $E$. This means that the general expression for the stress at a site $i$ in $E$ in a direction $\theta$ is given by

$$
\left(\sigma_{\theta, i}\right)_{E}^{\prime}=\left[\left(\sigma_{\theta, i}\right)_{E}+\rho_{\theta, i}\right] \text {. }
$$

The concept of a perturbation to the continuum stress field is based on the observation that, while on a macroscopic scale the continuum approximation can be used to approximate the stress field, at the grain scale, grains have a variety of shapes and sizes and the loading of a particular grain depends on its unique geometric relationship to its neighboring grains. The physical nature of $\rho$ can be understood by considering the grains shown in Figure 4.1-4. Figure 4.1-4 a shows a circular grain loaded in diametric compression, similar to the loading used in the Brazilian tensile test. Figure $4.1-4 \mathrm{~b}$ shows a tabular grain that has fractured due to bending. The analytic solution for the circular geometry (setting the disk diameter equal to 1) predicts that at the centroid of this grain a tensile stress with magnitude

$$
\sigma_{t}=\frac{2 \sigma_{1}}{\pi}-\sigma_{2}
$$

is generated perpendicular to the diameter in compression. If this geometry is embedded in an uncracked continuum such as $E$ and site $i$ represents the center of the circular grain, then the perturbed stress, $\sigma$, in the $\sigma_{2}$ direction in $E$ is given by equation 4.5 , which can be written as

$$
\sigma_{t}=\sigma_{2}-\rho_{i}
$$

where $\rho_{i}=2 \sigma_{1} / \pi$ for this particular geometry. This example shows how $\rho$ can be used to represent tension in a circular grain under diametric compression. 


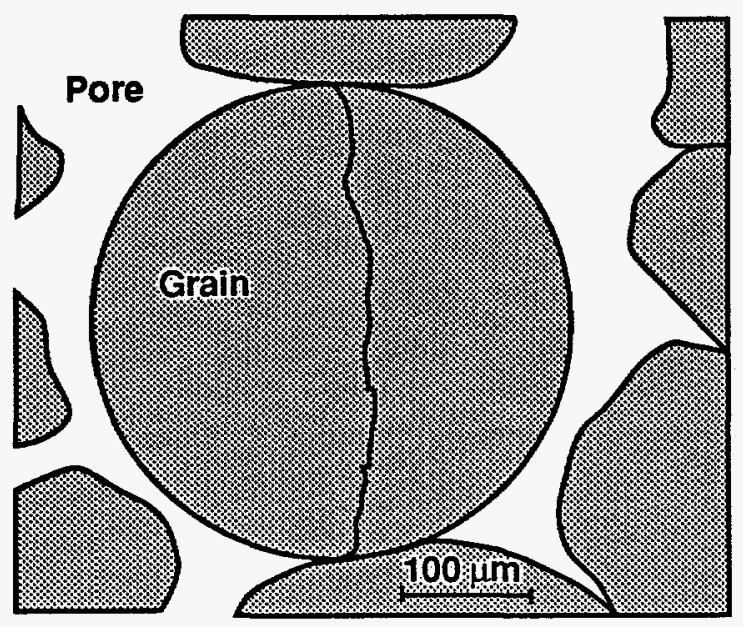

Brazilian loading a

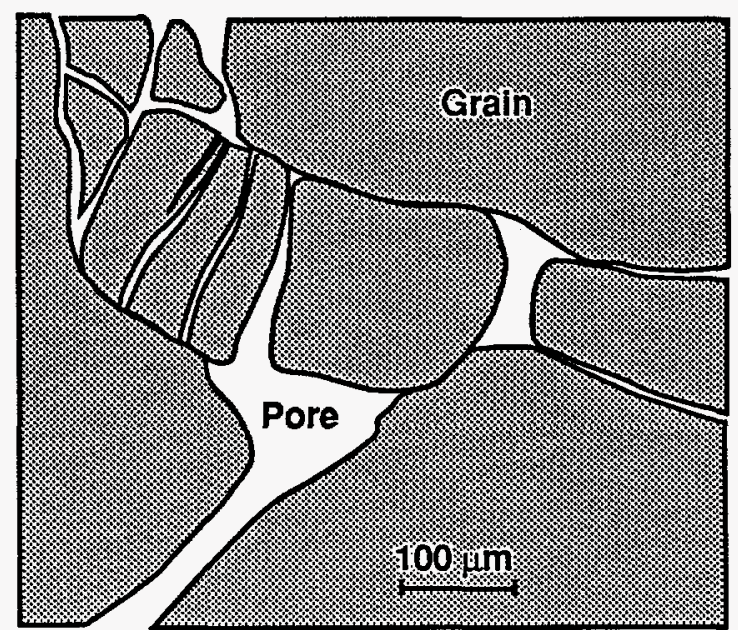

Bending

b

Figure 4.1-4. Examples of localized tensile fracture in a compressive stress field: a) a circular grain In diametric compression, b) a tabular grain loaded in bending (adapted from Zheng, 1989). 
In rocks there is wide variation in grain geometry, packing, cementation, and loading. Some grains may have induced tensions and others induced or amplified compressions. Thus, a general formulation of $\rho$ is required. The following criteria were used to formulate an expression for the stress perturbation factor:

(1) The stress perturbation is defined to act in the continuum component of the stress field.

(2) Stress equilibrium must be preserved (this is a central requirement for any model).

(3) Both local tension and compression must be simulated. (This is to accommodate the different types of grain loadings that may be present in a heterogeneous microstructure.)

(4) The perturbation should be proportional to the continuum stress difference at the site. This is because many fracture mechanics models use the stress difference as a basis for formulating stress intensity factors for extensile cracks in compressive stress fields (Kemeny and Cook, 1991).

(5) The perturbation should act in the direction perpendicular to the maximum local principal continuum stress at the site. (This is required by the crack-formation algorithm discussed in the next section.)

Given these criteria, the perturbation is introduced in the direction of the minimum principal stress in $E$. This direction is given by the angle $\phi$, and the expression for the perturbed stress at site $i$ in $E$ is

$$
\left(\sigma_{\phi, i}\right)_{E}^{\prime}=\left[\left(\sigma_{2, i}\right)_{E}+\rho_{i}\right]
$$

where $\rho$ is given by

$$
\rho_{i}=m_{1}\left[\left(\sigma_{1, i}\right)_{E}-c_{2}\left(\sigma_{2, i}\right)_{E}\right] \text {. }
$$

This formulation includes a stress difference multiplier, $m_{1}$, that is distributed in an interval between $\pm m_{1}$ with mean of zero. $c_{2}$ is a constant determined by the user (generally, $c_{2}=1$ ). The magnitude of $\rho$ at site $i$ is determined by the stress difference at the site and by the magnitude and sign of $m_{1, i}$. The stress perturbation is always applied in the direction of $\left(\sigma_{2, i}\right)_{E}$ which is 
given by angle $\left(\phi_{i}\right)_{E}$ and is perpendicular to the direction of the local maximum principal stress and of any new cracks.

Now the azimuth of the incipient crack at $i$ is determined from the direction of $\left(\sigma_{1, i}\right)_{E}$. For simplicity, only the components of the stress fields at $i$ in $E$ and $S$ that are perpendicular to this direction are used to evaluate the breaking rule at $i$.

Then we have, from equation 4.7 ,

$$
\begin{aligned}
\sigma_{B, i} & =\left(\sigma_{\phi, i}\right)_{E}^{\prime}+\left(\sigma_{\phi, i}\right)_{S} \\
& =\left(\sigma_{2, i}\right)_{E}+\rho_{i}+\left(\sigma_{\phi, i}\right)_{S},
\end{aligned}
$$

where $\sigma_{B, i}$ is the stress used to evaluate the breaking rule.

\section{Choice of Values for $m_{1}$}

The purpose of this section is to provide insight into how the ranges of values of $m_{1}$ used in this study were selected. It is instructive to consider specific cases of grain geometry and loading. One condition that provides insight is the situation where a circular grain (in two-dimensional cross section) is loaded in diametric compression, i.e., the Brazilian loading geometry. The tensile stress at the center of such a grain is given by equation 4.5 above. Then we have

$$
\sigma_{t}=-\left(\frac{2 \sigma_{1}}{\pi}-\sigma_{2}\right)=-\left(0.637 \sigma_{1}-\sigma_{2}\right) \text {. }
$$

This simple analysis shows that for a circular grain loaded in diametric compression a value of $m_{1}=0.637$ is appropriate when $\sigma_{1} \gg \sigma_{2}$.

A similar calculation can be done for the case of a clamped beam. In this geometry we have tensile stress at the end of a beam with unit thickness $T$ equal to

$$
\sigma_{t}=-\left(\frac{w L^{2}}{2 \mathrm{~T}}-\sigma_{2}\right) \text {. }
$$

where $w=$ weight and $L=$ length of the beam.

In this study we set $\omega=\sigma_{1}, L=l_{c}=1=$ lattice constant, and $\mathrm{T}=1$, as above. Thus we find that 


$$
\sigma_{t}=-\left(\frac{\sigma_{1}}{2}-\sigma_{2}\right)=-\left(0.5 \sigma_{1}-\sigma_{2}\right)
$$

and in this case $m_{1}=-0.5$ when $\sigma_{1} \gg \sigma_{2}$.

This evaluation shows that for these geometries values in the range 0.5 to 0.7 are possible for $m_{1}$. It is then assumed that for every grain oriented to produce a tensile stress concentration, there will exist a grain oriented to produce an equivalent compression, and using a value of $m_{1}$ with an opposite sign will account for this grain. Based on this simple analysis and examination of examples presented by Zheng (1989), Hadley (1976), and others, values of $m_{1}$ in the range -1.0 to 1.0 were chosen for this study. This is discussed further in Section 5.2.

This formulation defines a general model where two parameters, $T_{g}$ and $m_{1}$, are used to simulate heterogeneity in the strength and geometry of grains in rock.

\subsubsection{Formation of Cracks}

Formation of cracks is a key aspect of the field-theory model; three topics regarding crack formation are discussed in this section. First, the breaking rule is explained to show how the model decides which sites to break. Second, the superposition analysis given in Section 4.1.1 is extended to simulate the opening of extensile cracks under compressive stress, and the formation of cracks in $R$ using superposition of stresses in $E$ and $S$ is presented. Finally, the need for annealing behavior in the stress perturbation factor is explained, and a technique for annealing is presented.

\section{Breaking Rule}

The purpose of a breaking rule is to decide which sites break and at what conditions and in what order are they broken. In the field-theory model a site $i$ is said to break if the stress in the $\left(\phi_{i}\right)$ direction at that site in $R$ is tensile and is greater in magnitude than the tensile strength assigned to that site. The criterion for breaking is then

$$
\sigma_{B, i} \leq T_{g, i}
$$


where tension is negative and $\sigma_{B, i}$ is given by equation 4.9 . The complete expression is given by

$$
\left[\left(\sigma_{2, i}\right)_{E}+\rho_{i}\right]+\left(\sigma_{\phi, i}\right)_{S} \leq T_{g, i}
$$

where $T_{g, i}<0$.

When stress conditions at site $i$ meet criterion 4.14, the site cracks and a crack is formed in $R$.

If there are no cracks in $S$, the contribution from $S$ is zero and equation 4.14 can be rewritten as

$$
\rho_{i} \leq T_{g, i}+\left(\sigma_{2, i}\right)_{E},
$$

and using the complete expression for $\rho_{i}$ (equation 4.8) gives

$$
m_{1, i}\left(\sigma_{1, i}-c_{2} \sigma_{2, i}\right) \leq T_{g, i}+\left(\sigma_{2, i}\right)_{E}
$$

This shows that during the initial loading, the distributions of $T_{g}$ and $m_{1}$ dominate site cracking. This breaking rule is discussed in more detail in Section 4.4 .

\section{Formation of Cracks Using Superposition}

The analysis presented in Section 4.1.1 shows how superposition can be used to simulate opening a crack in a material under tension. In this section this analysis is extended to opening of a crack in a material under compression by invoking the local stress concentrations caused by heterogeneity in the shape and loading of grains, and employing the stress perturbation factor developed in Section 4.1.3.

Figure 4.1-3 shows the stress decomposition of the region of interest $R$. A crack is formed at a site $i$ in $R$ when $R$ is in compression, by inserting a crack at site $i$ in $S$ and opening the crack with a crack-opening traction $\left(\sigma_{c, i}\right)_{S}$ that acts on the internal surface of the crack and in the direction of $\rho_{i}$. This traction has magnitude

$$
\left|\left(\sigma_{c, i}\right)_{S}\right|=\left|\rho_{i}\right|
$$

Once a site is chosen to be cracked or broken, a crack is introduced as follows. The stressfree surface of the crack at site $i$ in $R$ is formed by superposition of the stresses on a crack inserted at site $i$ in $S$ and the stresses at site $i$ in $E$. 
It is useful to consider a simple application of this method, such as the circular grain loaded in diametric compression. It was shown above that for this geometry a tensile stress is generated along the diameter of compression. This problem can also be analyzed using the superposition technique discussed above. Figure 4.1-5 shows a circular disk under compression. The stress in a $\operatorname{disk} R$ is decomposed into that in an elastic continuum disk, $E$, and a cracked elastic disk, $S$. The stress along the incipient crack surface in $E$ is given by equation 4.6 above, but the crack is held closed by cct's equal in magnitude but opposite in sign to $\sigma_{t}$. S does not contribute to the stress field as all surfaces are unstressed. When $\sigma_{1}$ is raised sufficiently that $\sigma_{t}$ is greater than the tensile strength of the grain, then crack-opening tractions, $\sigma_{c, i}=\rho_{l}$, are inserted into the crack in $S$ and this crack is opened and superposition is used to form the stress-free surface of the crack in R.

A detailed description of the crack-formation algorithm in a more general geometry is presented in Figure 4.1-6. When a site $i$ is broken, identical cracks are inserted at $i$ in both $E$ and $S$. The orientation of these cracks is determined by the orientation of the maximum principal stress at site $i$ in $E$. Note that $\rho$ acts in the direction normal to the maximum principal continuum stress; thus, $\rho$ acts normal to the crack surface. Furthermore, $E$ is an elastic continuum, so the crack in $E$ is not allowed to open, and must be held closed by crack-closing tractions that are equal in magnitude but opposite in direction to the perturbed stress at $i$. In contrast, the crack inserted at $i$ in $S$ is opened, but as the far-field or boundary stresses on $S$ are zero, a traction normal to the crack surface is required to open the crack. The crack in $R$ is then formed by superposition of these two cracks, and because $R$ simulates the physical rock of interest, the surface of the crack in $R$ must be stress-free. To meet this condition, the magnitude of the crackopening traction at $i$ in $S$ is set equal to that of the crack-closing traction in $E$. The effect of the crack-opening traction in $S$ is to simulate the release of the crack-closing tractions in $E$. The opening of the crack in $S$ has the desired effect of dissipating strain energy in $S$.

After the crack is inserted in $S$, the stress fields in $S$ and $R$ are recalculated and the breaking rule is re-evaluated for unbroken sites. It is important to note that the traction applied normal to 


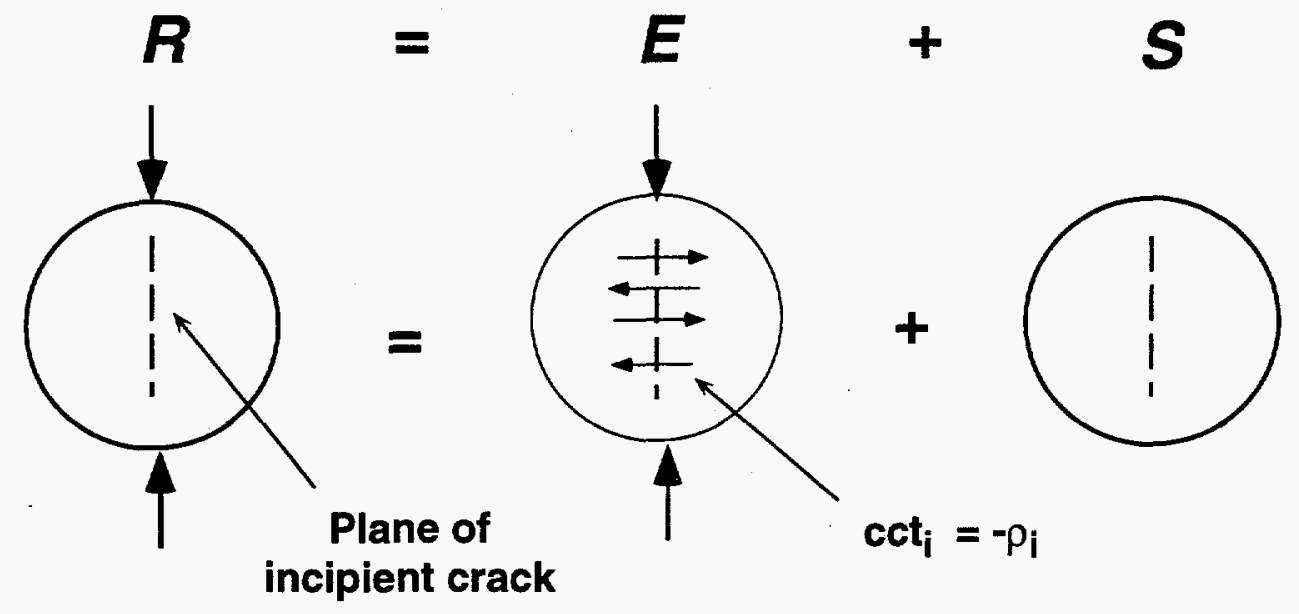

a) stresses on the plane of an incipient crack
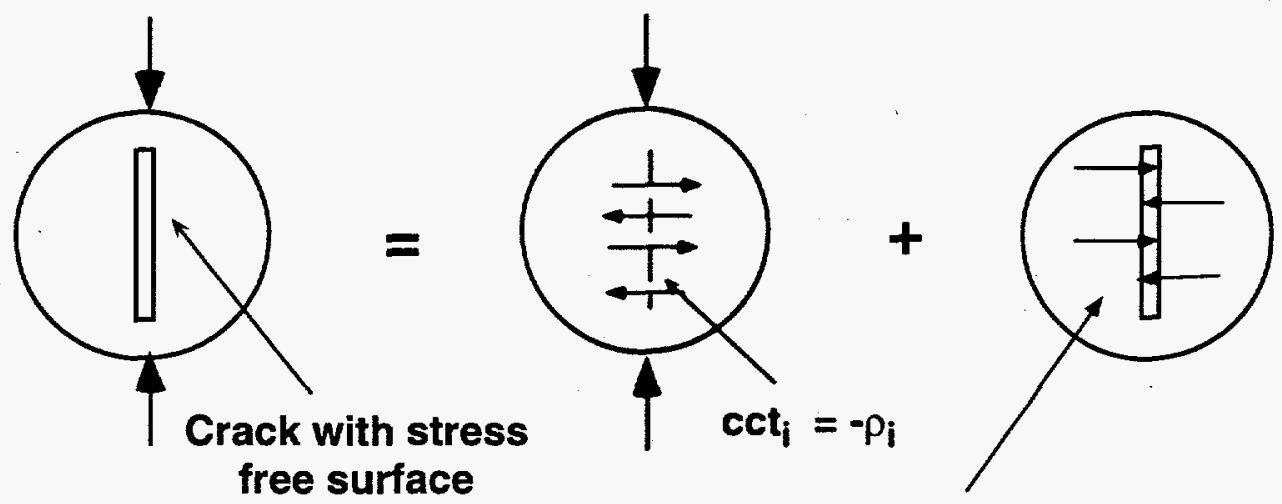

$$
\left(\sigma_{\mathbf{c}, \mathbf{i}}\right)=\rho_{\mathbf{i}}
$$

b) formation of stress free crack surface in $R$ using superposition of stresses in $E$ and $S$.

Figure 4.1-5. Analysis of crack formation in the brazilian geometry using superposition. 
Total stress
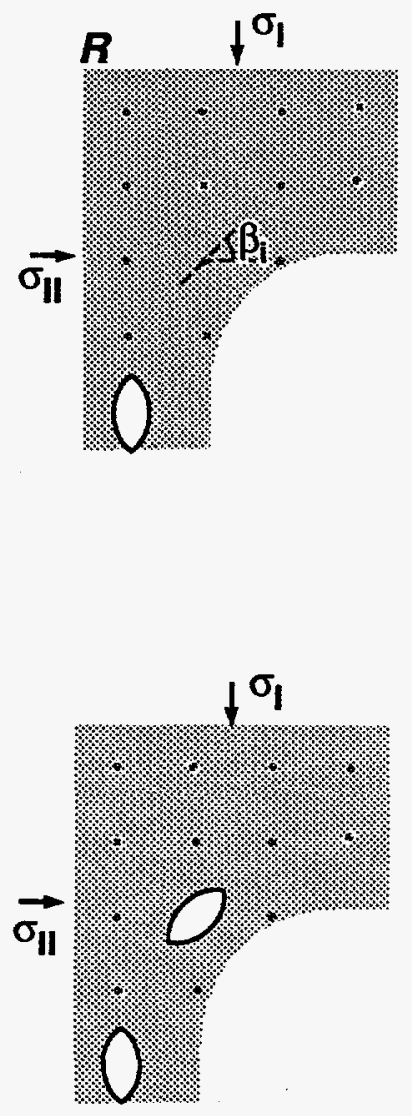

Elastic continuum

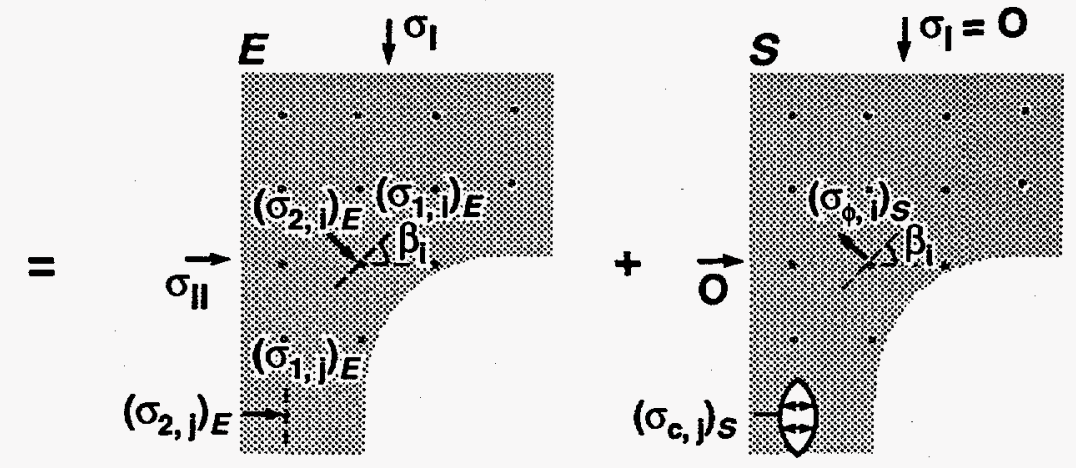

$\left(\sigma_{B, i}\right)_{R}=\left(\sigma_{2, i}\right)_{E}+\rho_{1}+\left(\sigma_{\phi, i}\right)_{S}$

Grain i breaks if $\left(\sigma_{\mathrm{B}, i}\right)_{R} \leq T_{g, i}$

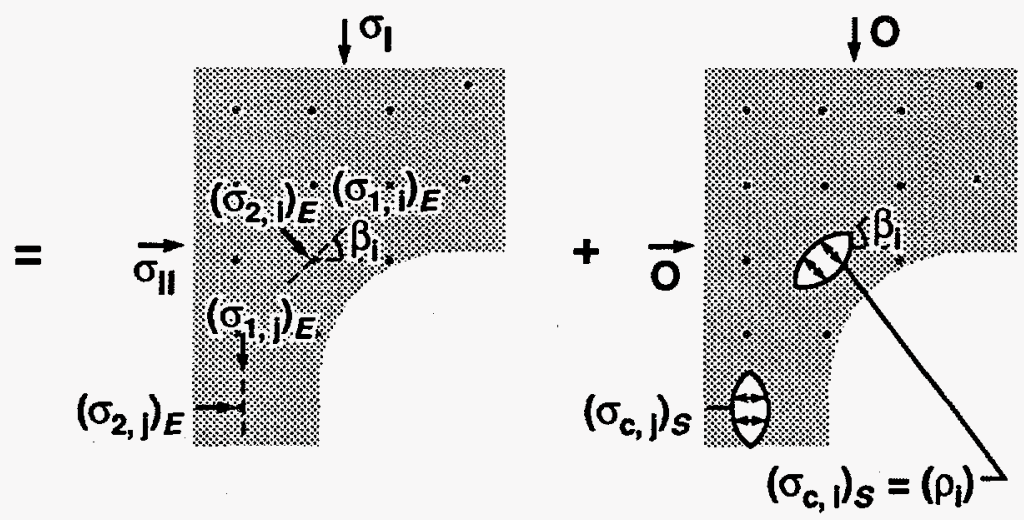

Figure 4.1-6. Detalled description of stress decomposition for crack formation in field-theory model. 
the crack surface at $i$ in $S$ can be a crack-opening traction or a crack-closing traction depending on the sign of $\rho_{i}$. In most cases this is a crack-opening traction, but occasionally the $\left(\sigma_{\phi, i}\right)_{S}$ term in equation 4.14 dominates the equation and a site breaks with $\rho$ acting as a crack-closing traction. In this case, the crack in $S$ still opens due to the stresses generated by other cracks, and $\rho_{i}$ serves to reduce the crack aperture. The numerical code used to implement the algorithm checks the apertures of the cracks and does not allow negative apertures. However, none has been observed.

\section{Annealed Heterogeneity in $\rho$}

The stress perturbation factor, $\rho$, is designed to reflect the microscale heterogeneity in the shape and loading of grains in a rock. Moreover, when cracks are introduced into the rock they may affect these local stress concentrations. That is, introduction of a crack may affect the loading geometry of grains in the vicinity of the crack. For instance, consider two neighboring grains aligned along an azimuth $\theta$. Grain 1 has a compressive loading geometry in the direction perpendicular to $\theta$; if a crack with azimuth $\theta$ is introduced at Grain 2, then Grain 1 will be subjected to tensile stress (stress intensity factor), acting in the direction opposite to the initial compression, that may override the initial compression. This simple example shows how as cracks develop in a material the stress concentrations on a given grain may become more correlated with the stress field associated with the cracks. Because introduction of cracks changes the microstructure, the stress perturbation, $\rho$, is formulated as an annealed parameter, which means that the stress perturbation at a site may change as cracking progresses. This allows the model to reflect the evolution of the microstructure in a material as cracks develop. Annealed behavior of $\rho$ is included in the field-theory model by setting the sign of $m_{1}$ at each site $i$ to be the same as the sign of the stress component $\left(\sigma_{\phi, i}\right)_{S}$ at a site $i$ in $S$. That is, the magnitude of $m_{1}$ for a site is constant throughout a simulation, but the sign may change. When a simulation is started, site values for $m_{1}$ are obtained from a random number generator, with a distribution specified by the user. No changes are made to the $m_{1}$ values while there are no cracks in $S$. However, when 
cracks are introduced into $S$, the stress values in the $\left(\sigma_{\phi, i}\right)_{S}$ direction are computed at all uncracked sites in $S$. The signs of $m_{1, i}$ and $\left(\sigma_{\phi, i}\right)_{S}$ are compared, and if they are different, the sign of $m_{1, i}$ is changed to match the sign of $\left(\sigma_{\phi, i}\right)_{S}$.

Figure 4.1-7 illustrates why annealing of $m_{1}$ is necessary and how it is accomplished.

Figure 4.1-7a shows a uniform stress field in a region of interest before any cracks are introduced. Figure 4.1-7 $\mathrm{b}$ shows the initial values of $m_{1}$ for sample sites $i-o$ on a square lattice. Positive values indicate that the stress perturbation is compressive, and negative values indicate that the perturbation is tensile, so that sites $k, l, m$ are more likely to break and $j$ and $n$ are less likely. Figure 4.1-7 c shows the local stress field that results when site $l$ is cracked. The opening of the crack at $l$ imposes a lateral compression (black) on sites $k$ and $m$, and a lateral tension (white) on sites $j$ and $n$. Examination of the initial values of $m_{1}$ for sites $k$ and $m$ shows that they are more likely to crack than sites $j$ and $n$. This will cause the crack to propagate to the side rather than along its axis. This is clearly unrealistic.

The annealed values of $m_{1}$ are shown in Figure 4.1-7 d. The sign of these values has been changed to be consistent with the local stress field associated with the cracks. Now sites $j$ and $n$ are more likely to crack and the crack is more likely to propagate along its axis than to the side. Note that the magnitude of $m_{1}$ does not change, so if $m_{1, i}$ is close to zero, stress difference will have a small role in cracking that site, no matter what the sign of $m_{1}$ is.

The need for annealed disorder in the stress perturbation term became apparent during trials with narrow distributions of geometric and site strength disorder. In some of these trials, two long converging cracks would fail to unite due to one site remaining uncracked, while sites to the side of the fractures would crack. This indicated a high compressive stress perturbation at the unbroken site and high tensile stresses at the sites adjacent to the fractures. Clearly any initial compressive perturbation at the initial site would have become a tensile perturbation in a real material. 
$\sigma_{1}$

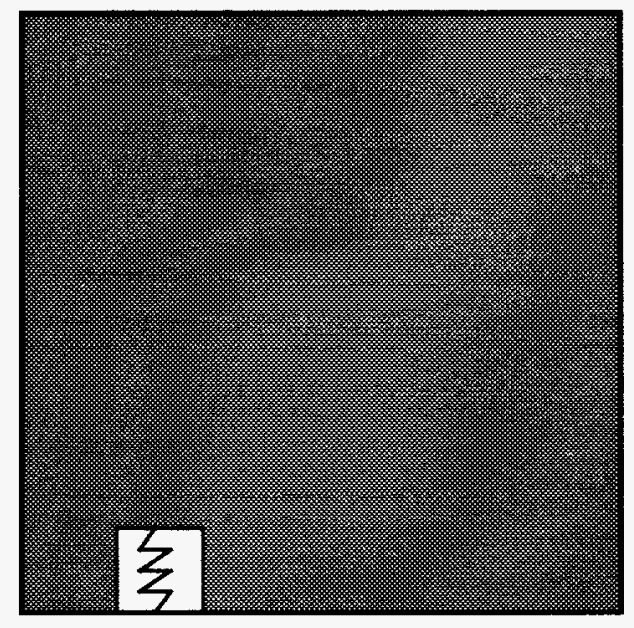

a

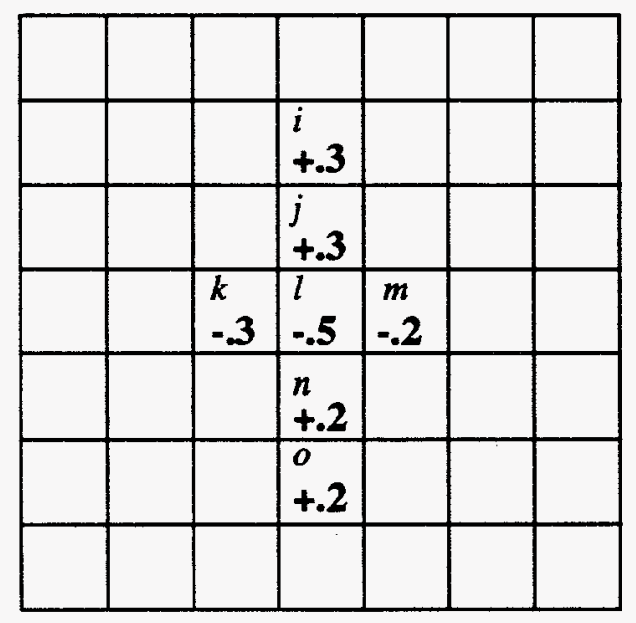

b

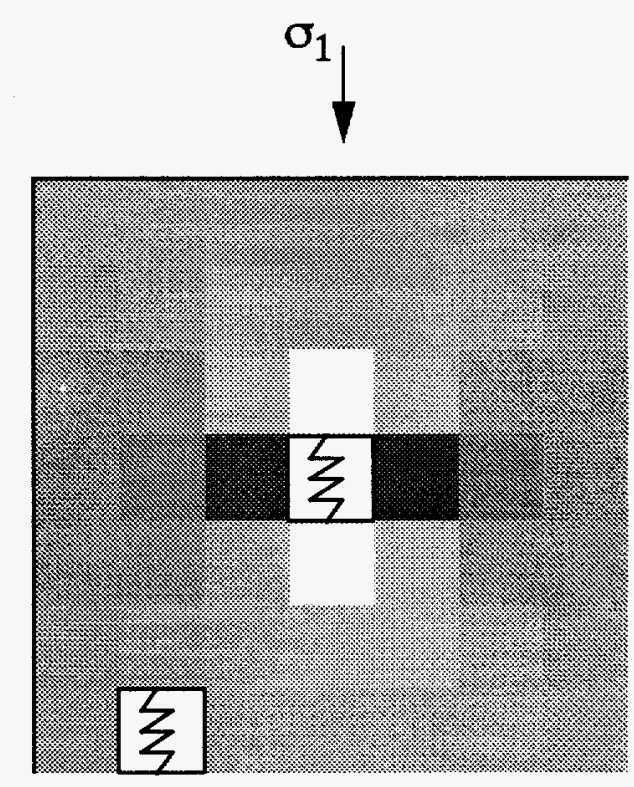

c

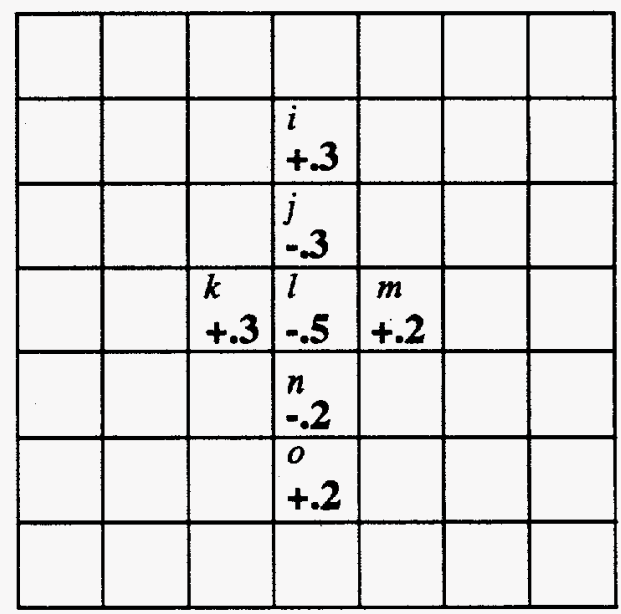

d

Figure 4.1 - 7 Illustration of annealing of $m_{1}$ parameter in field-theory model. (a) initial stress field, (b) initial $m_{1}$ values for selected sites, (c) stress field after a crack is introduced, (d) annealed $m_{1}$ values. 
To ensure that the total contribution of the stress perturbation term, $\rho$, is zero over the elastic array, all terms are summed after each new crack is formed, and each time the external stress conditions are changed. This is done using the expression

$$
\Pi=\sum_{i=1}^{N} \rho_{i},
$$

where $i$ is summed over all sites. This is necessary because the sign of $m_{1}$ at some sites in the array may have changed because of annealing, thus changing $\Pi$. If $\Pi$ is non-zero then it is divided by the number of sites, $N$, and as $\rho$ has approximately equal numbers of positive and negative values, this fraction is added to the stress perturbation at each site and $\Pi$ is then reevaluated. In practice, $\Pi$ is never exactly zero. However, trials done to assess the effect of changing the magnitude of the allowable deviation found that the model results are not sensitive to this parameter, and a total deviation of $0.5 \mathrm{MPa}$ is acceptable.

\subsubsection{Computation of Strain}

Macroscopic strain in $R$ is computed using the strain energy in $E$ and $S$ as follows. Strain energy, $U$, is defined as

$$
U=\frac{1}{2}(F \cdot d)=\frac{1}{2}(\sigma \cdot \varepsilon) .
$$

Then the strain energy for $R$ can be computed as the sum of the strain energies for $E$ and $S$.

That is,

$$
U_{R}=U_{E}+U_{S}
$$

Moreover, these two expressions can be combined to yield an expression for strain in $R$ as

$$
\varepsilon_{R}=\frac{U_{R}}{2 \sigma_{R}}
$$

The motivation for using this method of strain calculation is explained in detail in Section 4.4.1. 


\subsubsection{Changes in Boundary Conditions}

When a site breaks, equations 4.8 and 4.16 are used to determine the internal stress for the crack, and as the stress perturbation is based on the local stress difference at site $i$, the crackopening stress is dependent on the current level of applied stress. When the boundary or farfield stress conditions are changed this must be recomputed to reflect the new conditions. When the applied stress is changed, the changes in stress and deformation in $E$ are straightforward. The stress difference is re-evaluated for each site $i$ based on this new stress field in $E$. The new stress differences for the sites are used to update the crack-opening tractions for existing cracks in array $S$ following equations 4.8 and 4.16 .

The logic for this is that the analysis can always revert to a heterogeneous but purely elastic material by applying those values of crack-closing tractions that are exactly needed to keep cracks closed at any value of applied stress.

\subsubsection{Cluster Analysis}

One purpose of this study is to analyze the coalescence of cracks and the development of fractures. Percolation theory provides tools appropriate to this type of analysis, and in this study clusters of cracked sites are identified based on a nearest-neighbor criterion. This is site percolation. For a triangular lattice this implies each site has six nearest neighbors; for a square lattice, each site has eight nearest neighbors. When favorable geometries are simulated, the cluster structure can be analyzed for formation of a critical cluster (i.e., one that spans that lattice), and this can be associated with rock fracture. Further discussion of critical clusters and rock failure is presented in Section 4.4.4.

\subsubsection{Relationship of Microscale Heterogeneity to Matrix Deformation Modulus}

Simulation of crack formation, extension, and coalescence in $R$ requires that a deformation modulus $E_{m}$ be assigned to the matrix material in $R$. In this model it is assumed that the modulus of the matrix material in $E$ and $S$ has the same value at the matrix material in $R$. Thus, changes in 
the macroscopic modulus are only due to the introduction of cracks. The modulus of the matrix material remains constant throughout the simulation.

For a given simulation $\mathrm{E}_{\mathrm{m}}$ must be chosen to reflect the amount of microscale heterogeneity expected in the matrix of the material before the cracks are introduced. This implies that the effective modulus for the matrix material, $\mathrm{E}_{\mathrm{m}}$, is a function of both site strength and site geometry. This can be expressed as

$$
\mathrm{E}_{\mathrm{m}}=\mathrm{E}_{\mathrm{m}}\left(T_{g^{\prime}}, m_{1}\right)
$$

and may be formulated as

$$
1 / \mathrm{E}_{\mathrm{m}}=\left(1 / \mathrm{E}_{\mathrm{g}}\right)+f\left(T_{g^{\prime}} m_{1}\right)
$$

- where $\mathrm{E}_{\mathrm{g}}$ is the modulus of the grains in the material and $f\left(T_{g^{\prime}} m_{1}\right)$ increases with increasing range of $T_{g}$ and/or $m_{1}$. The exact formulation of $\mathrm{E}_{\mathrm{m}}\left(T_{g^{\prime}} m_{1}\right)$ is beyond the scope of this study.

Moreover, results of the parameter-sensitivity analysis presented in Section 5.1 provide insight into the nature of the functional dependence.

The concept of the dependence of $\mathrm{E}_{\mathrm{m}}$ on microstructural heterogeneity is illustrated by considering application of the field-theory model to three very different rock types. First, consider simulation of strong, uncracked rock with low porosity and a homogeneous grain structure, such as a fused quartzite. This rock type has very uniform, well-cemented grains with little variation in composition or geometry. For this rock type, values of $E_{m}=140 \mathrm{MPa}, T_{g}=50 \mathrm{MPa}$, and $m_{1}$ uniform over \pm 0.05 are appropriate (Table 4.1-1). Thus, very high differential stresses are required to introduce cracks.

Table 4.1-1. Dependence of deformation modulus on heterogeneity in the matrix.

\begin{tabular}{lccc}
\hline Rock type & $m_{1}$ & $\begin{array}{c}T_{g} \\
(\mathrm{MPa})\end{array}$ & $\begin{array}{c}E_{\mathrm{m}} \\
(\mathrm{MPa})\end{array}$ \\
\hline Fused quartzite & \pm 0.05 & $50 \pm 0.0$ & 140 \\
Cracked quartzite & \pm 0.3 & $45 \pm 10$ & 100 \\
Sandstone & \pm 0.7 & $20 \pm 19.9$ & 50 \\
\hline
\end{tabular}


Next, consider a cracked quartzite. This type of rock also has a strong, well-cemented matrix with low porosity and homogeneous grain structure, with a few initial cracks. For this rock type, values of $E_{m}=100 \mathrm{MPa}, T_{g}=45 \pm 10 \mathrm{MPa}$, and $m_{1}$ of \pm 0.5 may be appropriate. Finally, consider a poorly sorted but well-cemented sandstone with moderate porosity. For this rock type, values of $\mathrm{E}_{\mathrm{m}}=50 \mathrm{MPa}, T_{g}=20 \pm 19.9 \mathrm{MPa}$, and $m_{1}$ of \pm 0.7 are appropriate.

The field-theory model was formulated to investigate how different types of microscale heterogeneity affect macroscopic behavior, and specifically the model allows evaluation of the effect of introducing cracks on the behavior. In this study the model is not used to simulate rocks with low heterogeneity, as these approximate an elastic continuum and are better studied using a continuum model. The field-theory model can be configured to simulate these materials by reducing the amount of heterogeneity incorporated to near zero. Note that the parametersensitivity analysis presented in Section 5 was conducted with $E_{m}$ held constant at $70 \mathrm{MPa}$ for all trials.

\subsection{Model Implementation Using Field Theory and the Boundary Element Method}

The model has been implemented using a field-theory approach, where the behavior of a site on the lattice is determined using rule-based criteria in concert with a stress field computed using the boundary element method (BEM). The boundary element method was chosen because it lends itself naturally to the superposition approach discussed above, and superposition is an essential part of the BEM formulation. The BEM method is elastic and thus equilibrium is preserved and solutions are unique. Moreover, proven codes are available for both static and dynamic analysis. In addition, using BEM it is extremely easy to input cracks and complex geometric boundaries, and changing boundaries such as those found underground.

This section provides detail on how this implementation was developed. First it is important to discuss where different parts of the model reside. The decomposition of stress in the model is shown in Figures 4.1-3 and 4.1-6. In the actual implementation of the model, region $R$ exists in 
field theory while regions $E$ and $S$ are formulated using boundary elements. This means that the parameters for region $R$ are stored in a variety of arrays that provide site strength $T_{g^{\prime}} m_{1}$, stress perturbation $\rho$, information on what sites are broken, angle and length of cracks at sites, stress and displacement components, etc. Moreover, while Figure 4.1-6 also shows that cracks are inserted in both $E$ and $S$, in practice cracks are only inserted in $S$.

The BEM formulation used in the model is a direct adaptation of the displacement discontinuity method (TWODD) given by Crouch and Starfield (1990). A description of the different codes that comprise the model is presented in Section 4.5 .

\subsubsection{Type and Number of Elements per Crack}

The approach outlined in the previous sections requires that the method of modeling cracks has the capability of inserting a crack at a site and opening the crack with a specified crack traction. The displacement discontinuity technique of BEM is well suited for this application. However, use of this technique in problems of cracking and crack propagation requires that the number and specific type of displacement discontinuity elements used be considered carefully. This is because the displacement discontinuity element has a fundamental limitation, as follows. The solution for the stresses and displacements in the body due to the displacement discontinuity element is only valid at points in the body located in the area outside a circle centered at the center of the element and with radius greater than one element length. This is illustrated in Figure 4.2-1. This figure shows that in order to estimate stress and displacement at sites neighboring a cracked site $i$, the crack at site $i$ must be composed of more than one element.

Standard displacement discontinuity elements are used in the field-theory model to form both boundaries and internal cracks at lattice sites. This element type was chosen because of it's simplicity and has recently been shown to provide the most accurate approximation to the behavior observed for cracks introduced in PMMA (Germanovich, 1994). Cracks at lattice sites are composed of three elements. The number of elements per crack and the type of elements relate directly to the accuracy of the boundary element approximation of the stress field; the decision to use three elements was based on preliminary evaluation of several options. These 


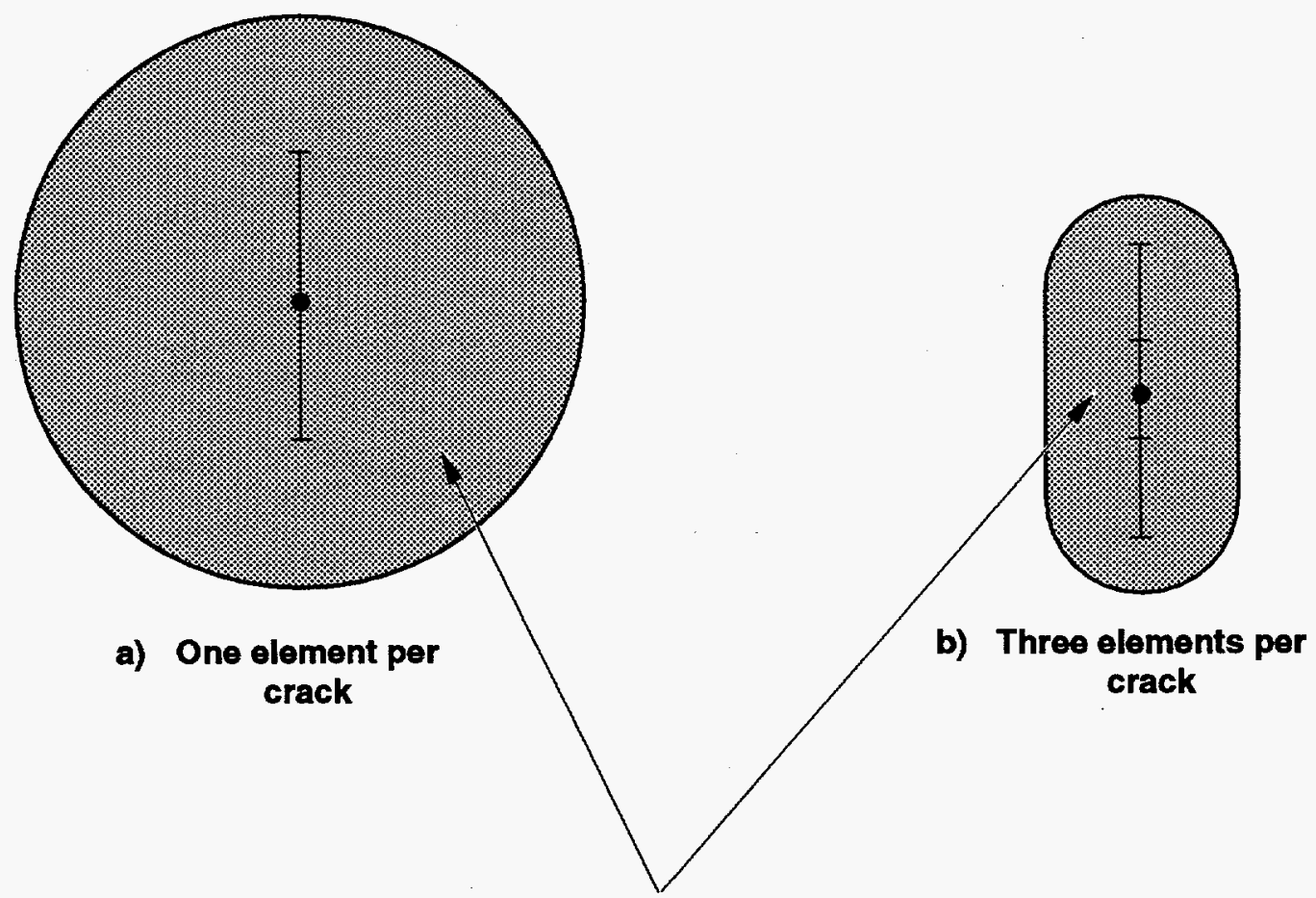

Region where BEM solutions are inaccurate

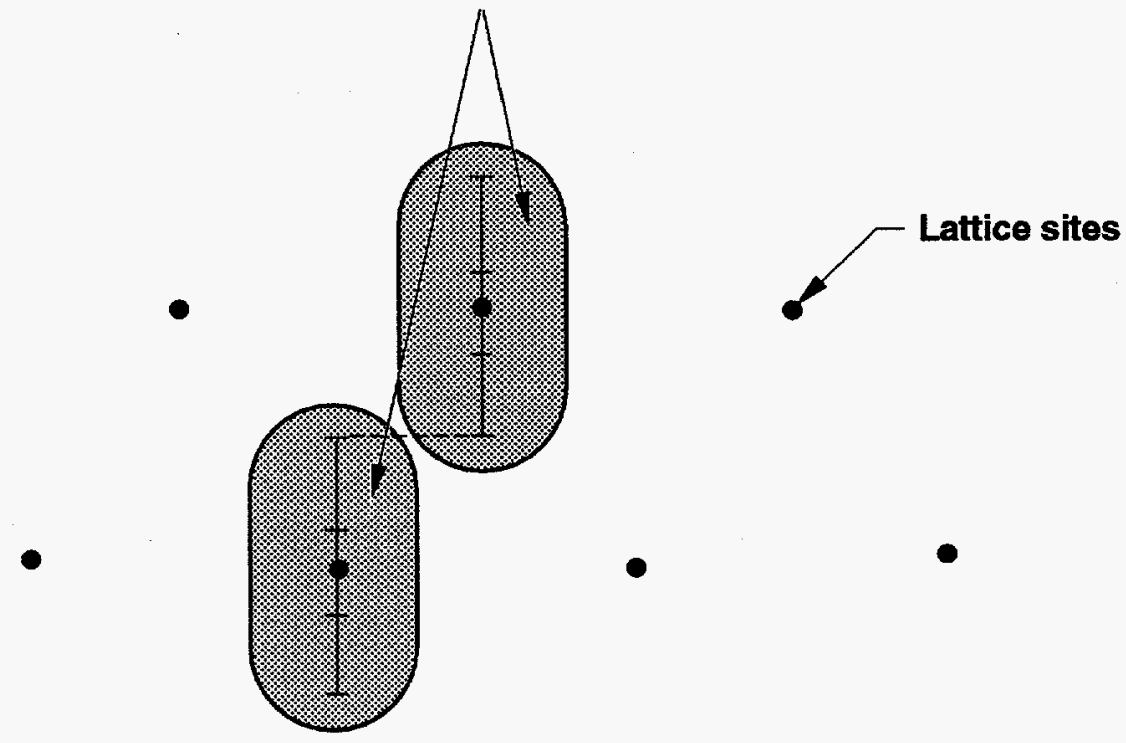

c) Three elements per crack

on a triangular lattice

Figure 4.2-1. Region where BEM solutions are inaccurate for three configurations. 
include the use of more than three elements per crack, use of crack tip elements, and use of higher-order elements such as linear or quadratic elements, thereby reducing the number of elements required, but increasing the complexity of the numerical code. Crouch and Starfield (1990) have evaluated crack tip elements by computing strain energy vs number of elements per crack. Their analysis shows that the savings due to the crack tip elements are realized when only a few elements are used, e.g., $<10$ elements, and that if 15 elements or more are used per crack, the two types of elements produce very similar results. For the application developed here, several elements are often linked together (see Section 4.3.2). Thus, for many of the element configurations, the performance of the code is not significantly enhanced by using the crack tip elements because a large number of elements are already used.

The main advantage of crack tip elements would be for isolated cracks at individual sites. A series of trials was conducted to evaluate the performance of the code using different numbers of elements per crack, and results for a $30 \times 60$ array that contained 50 cracks are shown in Figure 4.2-2. This figure shows the maximum compressive and tensile stresses observed in the array for cracks with between one and eight elements per crack; increasing the number of elements per crack beyond three has diminishing returns.

Another technique for improving the accuracy of the computation is to use higher-order elements, such as those developed by Crawford and Curran (1982). These linear or quadratic elements have the advantage that they are more stable, and provide a more reliable estimate of the stress field close to the element. However, use of these boundary elements would require a major effort in reprogramming and code verification. Moreover, it is important to recall that the purpose of the BEM computation in this code is to provide estimates of continuum stresses in complex geometries, and estimates of the stresses due to crack interaction, in addition to providing overall stress equilibrium. While attention must be paid to the limitations of the BEM, the purpose of this studyris to integrate continuum analysis with the concept of disorder in the material and not to get an exact estimate of stress in a cracked continuum. It is important to note that for fracture of rock, the absolute value of stress at a point in the rock is not a valid concept, 


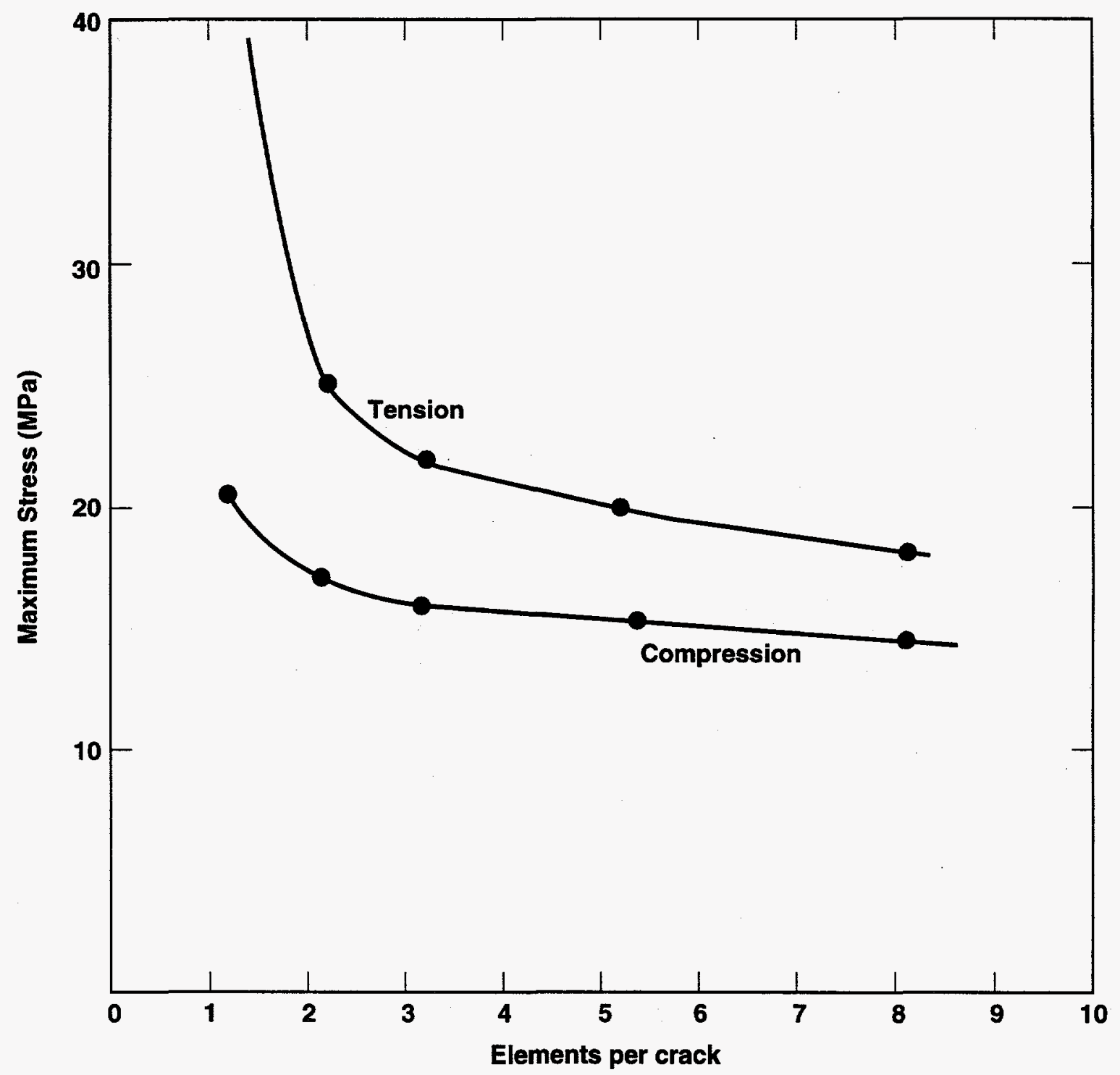

Figure 4.2-2. Maximum local tensile and compressive stresses computed for a simulated compression test vs. number of elements used per cracked site. 
due to the heterogeneity discussed earlier. In particular, the magnitude of the stress perturbation, $\rho$, introduced at any site is often greater than any error in stress approximation provided by the BEM. Thus, for the purposes of this study, great precision of the stress field is not required, and three elements per crack was judged to produce an acceptable and useful approximation to the stress field.

Conversations with Carl Renshaw (1993) reaffirmed the view that for the purposes of this study, the extensive amount of code development required to implement either the crack tip or higher-order elements was not justified, as it would not lead to significant improvement in the performance of the code.

\subsubsection{Crack Orientation}

The convention used in the BEM makes it important to keep track of element orientation when inserting cracks into the model. The boundary elements inserted into the field-theory model are oriented parallel to the local principal elastic stress component with the + side of the displacement discontinuity element on the right with respect to the far field or boundary stresses, as shown in Figure 4.2-3. For ease of computation the principal stresses were usually aligned along the $\mathrm{x}$ and $\mathrm{y}$ axes. However. due to a "zero- divide" condition, the numerical implementation of the BEM method as coded into the field-theory model is numerically unstable if boundary elements are oriented exactly parallel to the $x$ or $y$ axes. To avoid the numerical instability a small offset was introduced into the crack element orientation if the element was aligned parallel to an axis. Moreover, it is important to note that in real heterogeneous materials cracks are not exactly aligned with the stress field because of the microscale heterogeneity present in the material. The effect of small changes in crack orientation was evaluated as follows. For simulated uniaxial tests an offset was introduced into crack orientation for cracks precisely aligned along the $\mathrm{x}$ or $\mathrm{y}$ directions.

Two different algorithms for offset were evaluated. One was to offset the cracks by a constant amount of rotation, say a one-degree clockwise rotation. This is a straightforward approach and it was tested using $10 \times 30$ arrays in simulating laboratory compression tests, as 


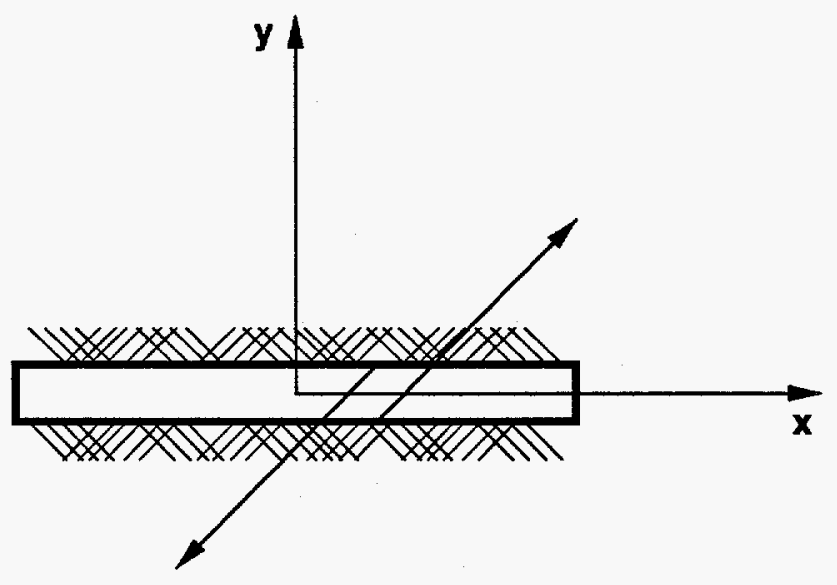

Figure 4.2-3 Stresses applied to crack surface. (after Crouch and Starfield, 1990) 
described in Section 4.4. Crack patterns for ten trials computed using such an algorithm are shown in Figure 4.2-4 a. This algorithm produces tight, well-organized clusters of cracks. However, the patterns all trend to the lower left / upper right. This implementation shows promising behavior, but the consistent stepping is unrealistic.

Another approach was to randomly perturb the orientation of the inserted crack within set limits. For instance, if the angle, $\beta$, of the principal elastic stress at site $i$ is such that $90+0.05<\beta$ $<90-0.05$, then a random number $\varepsilon$ can be chosen in the interval $(-1,1)$. If $\varepsilon>0$, the angle for site $i$ can be set to $90+0.05$. If $\varepsilon<0$, the angle at site $i$ can be set to $90-0.05$. Results of trials with this algorithm implemented are shown in Figure 4.2-4 b. These trials were computed using the same $m_{1}$ and site strength as the trials shown in Figure 4.2-4 a. This algorithm produced higher percolation thresholds (more sites broken when a critical cluster was formed) and higher values of deformation modulus (i.e., stiffer stress-strain behavior) than the trials with all cracks oriented in the same way. The crack patterns for the randomized trials are more realistic, and the consistent stepping to the lower left is not present, so this formulation is used in the subsequent analysis. However, this result shows how systematic rotational offset might be used to build anisotropy, cleavage, or bedding into a simulation.

\subsubsection{Computation of Strain Energy and Strain}

The method for computation of strain energy is presented in this section. This parameter is used in the field-theory model to describe the energy used in formation of cracks and to compute axial strain in simulated compression tests. One of the purposes of decomposing the stress field of $R$ into elastic continuum and crack interaction components is to provide a method for assessing the role of cracks and crack interaction in the processes that control macroscopic behavior and fracture. Strain energy is a parameter that can aid in understanding the processes of fracture. In particular, it can be used to provide information as to how damage is accumulating at a given stress condition and a given number of cracks. In addition, it can be used along with the applied stress conditions to estimate strain. In this section strain energy, $U$, is defined as 

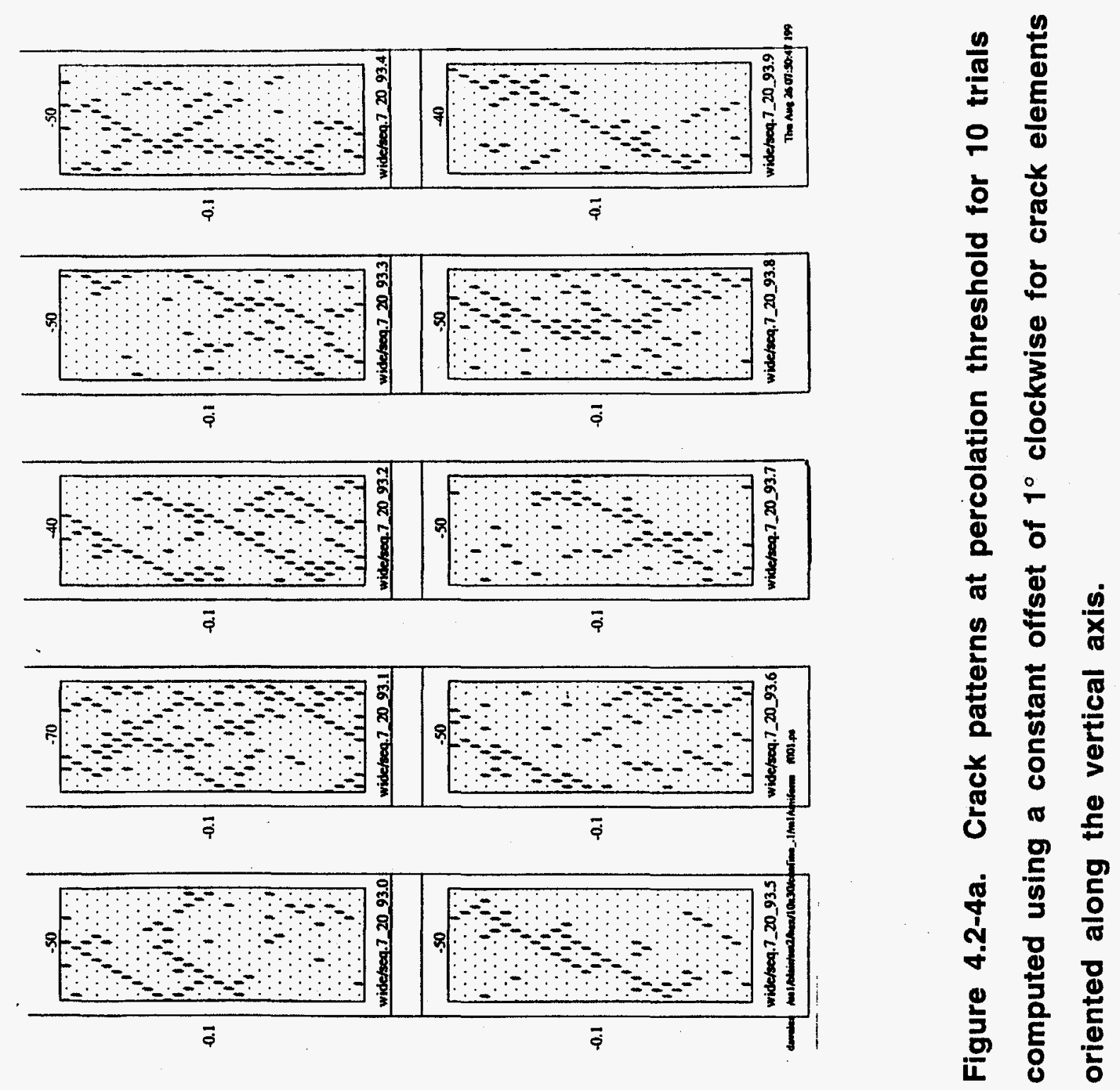

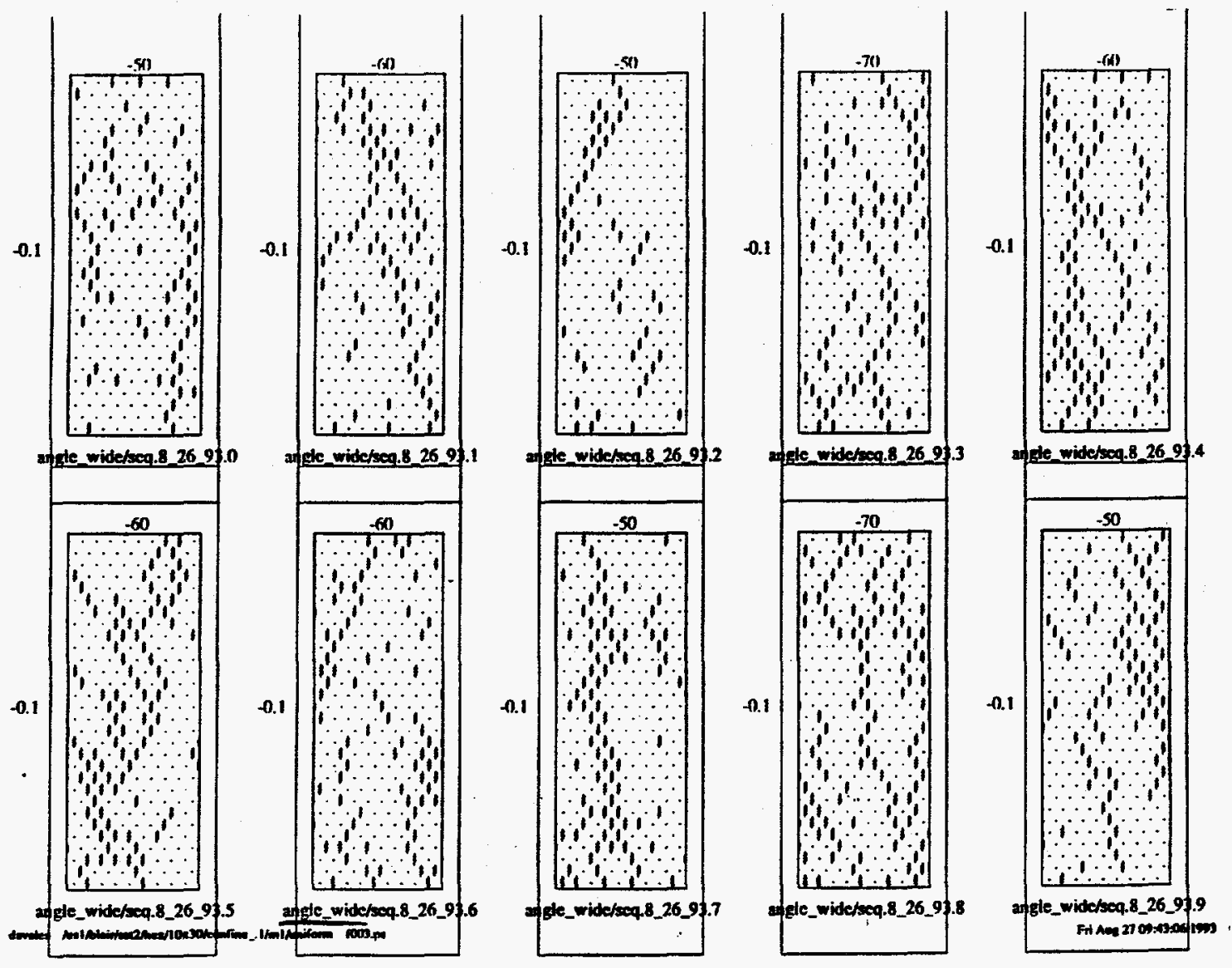

Figure 4.2-4b. Crack patterns at percolation threshold for 10 trials computed using a stochastic offset algorithm. See text for details. 


$$
U=\frac{1}{2}(\sigma \cdot \varepsilon)
$$

In this analysis the strain energy in region $R$ is decomposed into the strain energy components due to the elastic continuum, $E$, and the cracked elastic body, $S$, defined in Section 4.1. Then

$$
u_{R}=U_{E}+u_{S}
$$

$U_{E}$ can be computed analytically for a given stress field as

$$
U_{E}=\frac{1}{2}\left(\sigma_{1} \varepsilon_{1}-\sigma_{2} \varepsilon_{2}\right)
$$

Now the effective modulus $E_{m}$ is defined as

$$
\mathrm{E}_{\mathrm{m}}=\frac{\sigma_{1}}{\varepsilon_{1}}
$$

so $U_{E}$ can be written as

$$
U_{E}=\frac{\sigma_{1}^{2}}{2 \mathrm{E}}-\frac{\sigma_{2} \varepsilon_{2}}{2}
$$

For the cracked elastic body, $S$, the strain energy is due to the opening of cracks, and for a crack at site $i$ is given by

$$
\left(U_{i}\right)_{S}=\frac{1}{2}\left(\rho_{i} \varepsilon_{i}\right)_{S}
$$

where $\rho_{i}$ (the stress perturbation factor) is the crack-opening traction, and $\varepsilon_{i}$ is the strain in $S$ due to the crack at $i$.

$\rho_{i}$ is known, and the strain $\left(\varepsilon_{i}\right)_{S}$ is computed as follows. The crack at $i$ has a length $l_{i}$ and opens to an aperture $\delta_{i}$, creating additional area $\Delta A_{i}$, where

$$
\Delta A_{i}=4 \xi
$$

The total strain due to the opening of the crack at $i$ is then

$$
\left(\varepsilon_{i}\right)_{S}=\frac{\Delta A_{i}}{A}
$$

where $A$ is the area of the array.

Substituting 4.25 and 4.24 into 4.22 , the strain energy due to the crack at $i$ is given by

$$
\left(U_{i}\right)_{S}=\frac{1}{2}\left(\rho_{i} \varepsilon_{i}\right)=\frac{1}{2 A}\left(\rho_{i} l_{i} \delta_{i}\right) .
$$


The total strain energy in $S$ due to $n$ cracks is given by

$$
U_{S}=\left(\frac{1}{2} A\right) \sum_{i=1}^{n} \rho_{i} l_{i} \delta_{i} .
$$

As discussed in Section 4.1.4, crack traction, $-\sigma_{c, i}$ given by equation 4.16 , can sometimes be a crack-closing traction rather than a crack-opening traction. In this case equations 4.31 and 4.32 are inappropriate, as the traction does not operate through the opening displacement $\delta$, but instead serves to reduce $\delta$. Computation of the exact strain energy using equations 4.31 and 4.32 corrected for this effect is beyond the scope of this study. Moreover, a conservative estimate of $U_{S}$ can be obtained using only cracks where $\rho$ is crack-opening and neglecting cracks with crackclosing tractions. Because $U_{S}$ is only an indicator function, this revised estimate is adequate for the purposes of this study.

The expression in equation 4.32 provides a measure of crack strain energy at a particular level of applied stress, and as discussed in Section 4.1.4, $\rho$ is dependent on the applied stress and the stress difference at the site. A more useful quantity is the normalized crack strain energy $\bar{U}_{S}$. To normalize $U_{S}$, it is divided by the total strain energy in the system. Thus

$$
\bar{u}_{S}=\frac{U_{S}}{U_{R}}=\frac{U_{S}}{U_{E}+U_{S}} .
$$

This provides a measure of the partitioning of energy between the elastic continuum and the cracked body components of the strain energy in the system.

\subsection{Model Implementation on Lattices}

In this study simulations were conducted with sites located on both triangular and square lattices. In addition, perturbed or irregular versions of these basic lattices were also used. A typical triangular lattice is shown in Figure 4.3-1 a. The triangular lattice has the advantage of being isotropic and has been used for most of the trials discussed in this thesis. An example of a perturbed triangular lattice is shown in Figure 4.3-1 b. Similarly, examples of square and perturbed square lattices are shown in Figures 4.3-1 $\mathrm{c}$ and $\mathrm{d}$. These different lattices were used to determine the effect of heterogeneity in site location on the macroscopic behavior. 


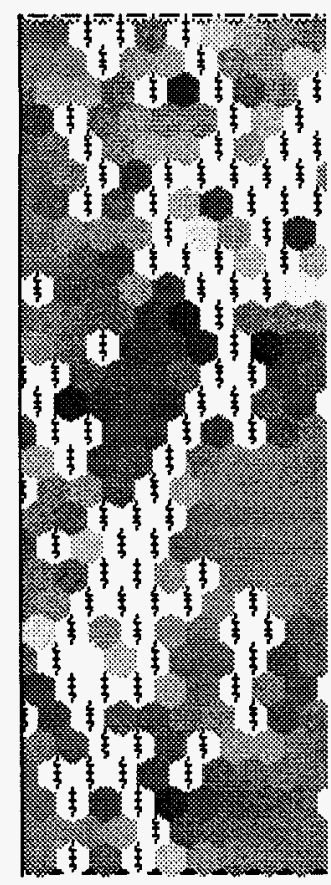

(a) triangular

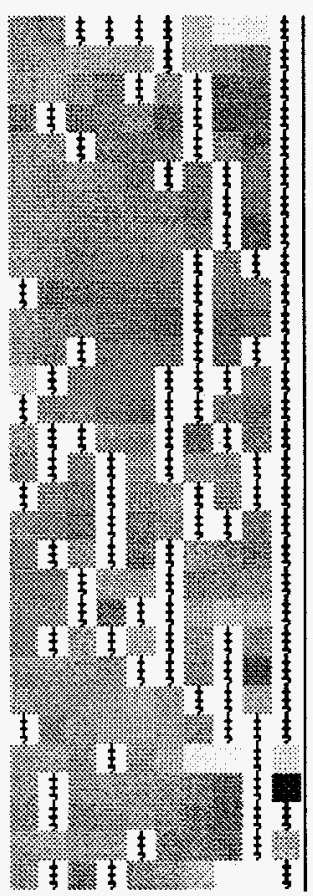

(c) square

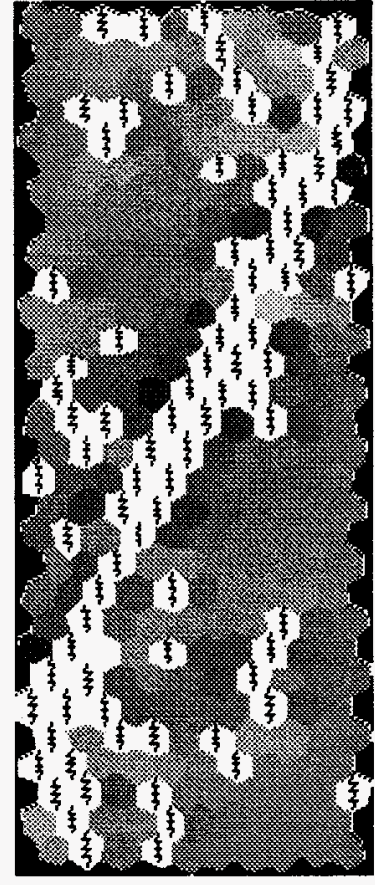

(b) perturbed triangular

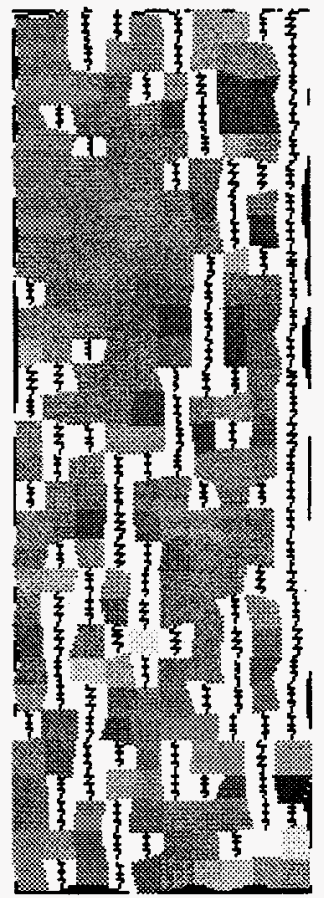

(d) perturbed square

Figure 4.3 - 1 Predicted crack patterns for regular and perturbed lattices. 
In the field-theory model, sites are assigned to the region to be analyzed and a portion of the area of the region is associated with each site so that the entire area of the region is covered. This is illustrated in Figures 4.3-1 a-d, which show that any point in the discretized region is associated with a lattice site. This is because in the field-theory model porosity is not incorporated explicitly; rather it is implicit in the assumptions made about the material. In particular, a pore is assumed to be a site with strength that is zero, or near zero. When regular lattices are used this concept is simple to implement. If the desired lattice is square, then a square grid is overlaid on the region and the centroids of the grid blocks become the lattice sites (see Figure 4.3-2 a). The square area associated with the site is then used as a convenient vehicle for displaying stress levels at the site. This can be done by coloring the square based on the stress level. If a triangular lattice is desired, then a hexagonal or honeycomb grid is overlaid on the region and the centroids of this grid become the lattice points or sites. With either of these schemes, the entire area is covered and any point located in the region can be associated with a centroid and thus with a crack site. This is important, because if a location cannot be associated with a lattice site then that location is defined as linear elastic and unbreakable. This criterion must also hold for the irregular lattices. Irregular lattices that meet this criterion were produced from the square and triangular lattices by perturbing the lattice sites and then constructing areas or domains associated with each site such that all points in the study area are assigned to a lattice site.

The derivation of an irregular quadrilateral lattice from a regular square lattice is illustrated in Figures 4.3-2 a and b. First a regular square lattice is produced with lattice constant $l_{c}$. Site $i$ is perturbed by moving it to a new location specified by a distance $r_{p, i}$ and an azimuth $\theta_{i}$. Values for $r_{p, i}$ and $\theta_{i}$ are chosen randomly from uniform distributions over the ranges $0 \leq r_{p, i} \leq l_{c} / 2$ and $0 \leq \theta_{i} \leq 2 \pi$. The perturbed sites then become the vertices of irregular quadrilaterals that cover the region of interest. The centroids of the quadrilaterals then become the new sites of the irregular lattice. The crack length for each site is then determined as follows. The orientation of the crack at a given site is taken to be parallel with the local maximum principal continuum stress at that site (angle $\beta_{1}$ ), as discussed in Section 4.1.4. A line with this orientation is drawn through the 


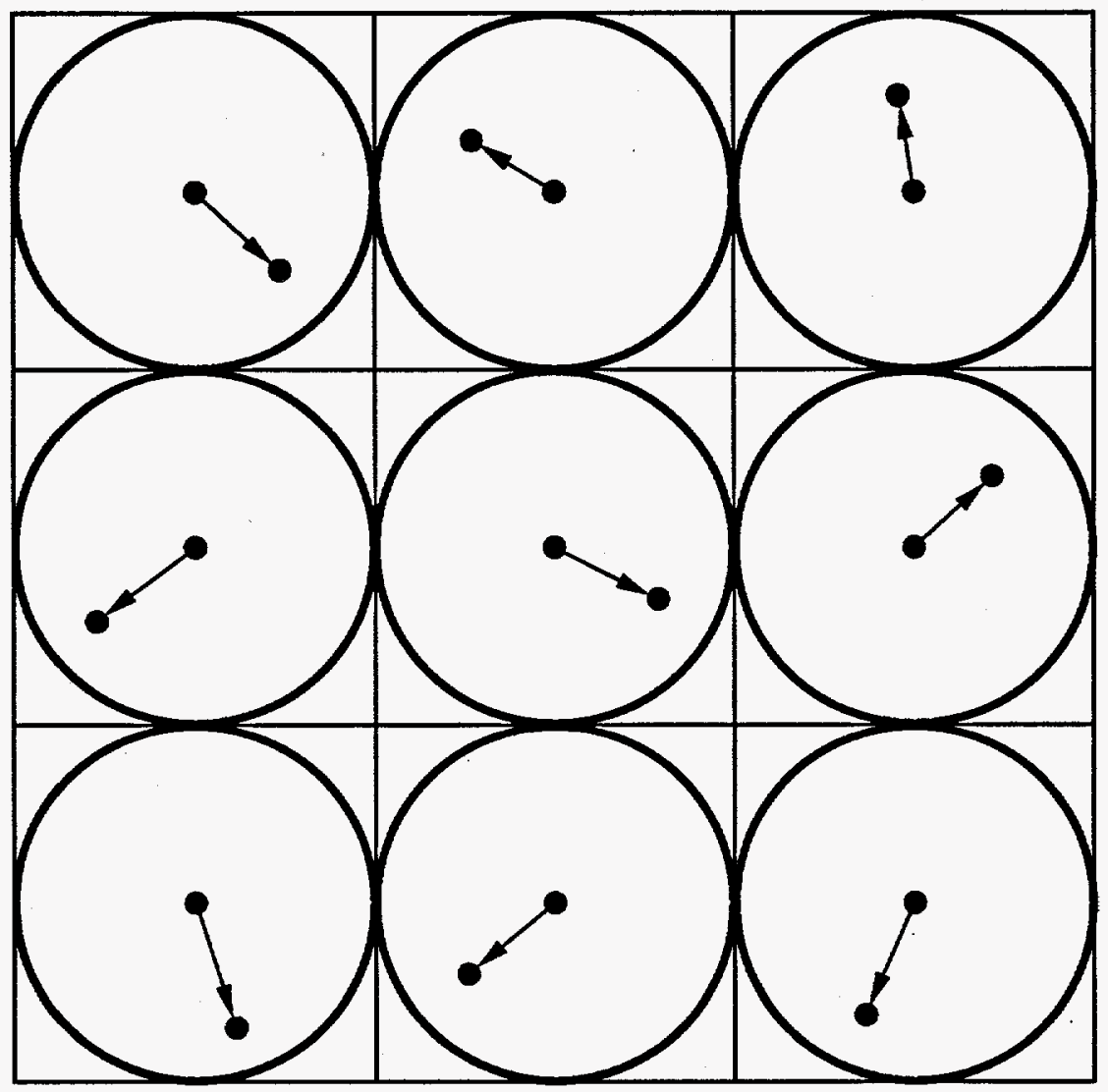

Figure 4.3-2. Production of perturbed square lattices

a) Original sites are relocated to new coordinates. 


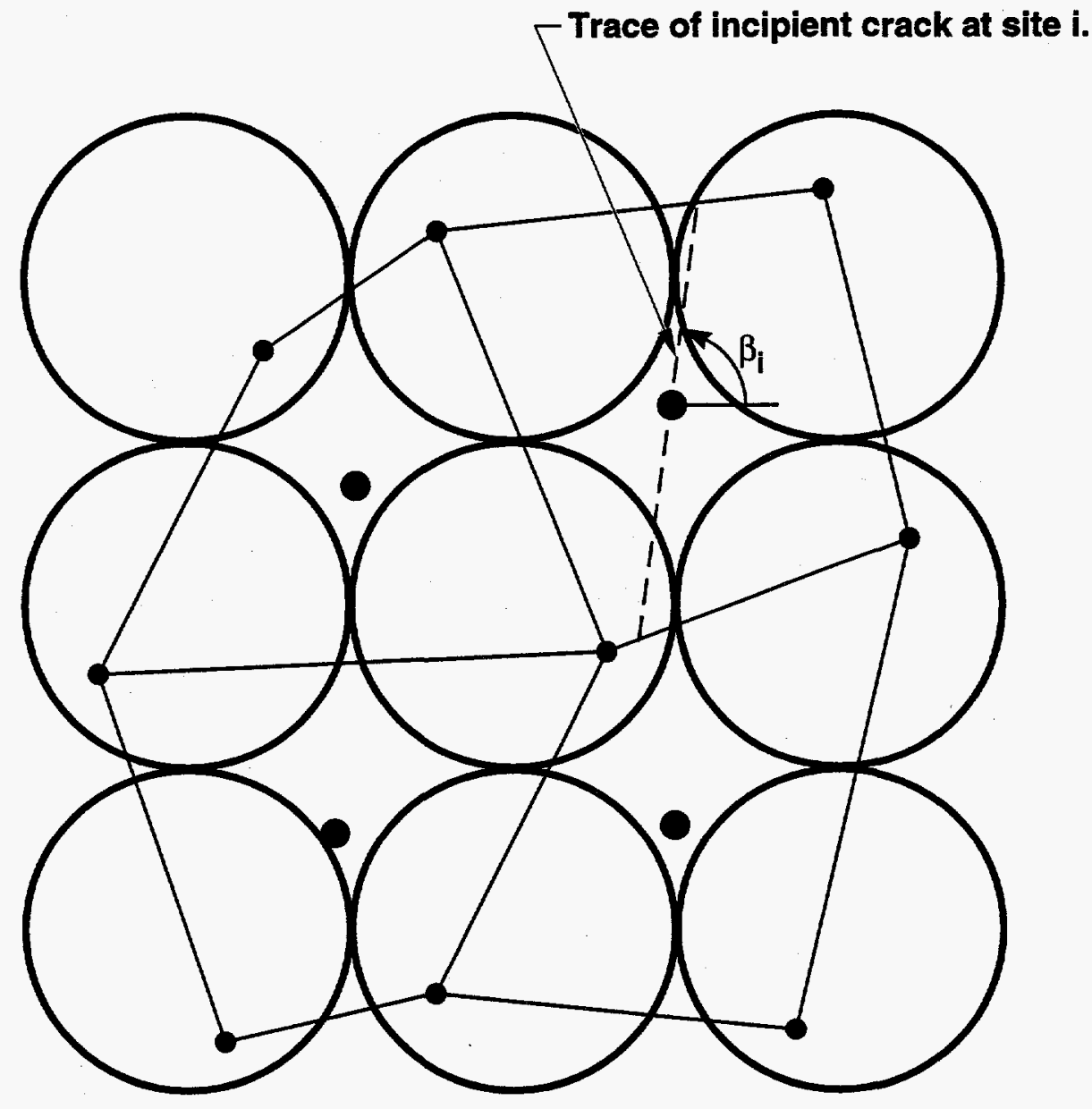

Figure 4.3-2b. Sites at new coordinates become vertices of irregular quadrilaterals. Center of mass of quadrilaterals become sites on the perturbed lattice. 
new site. The crack length is then determined by the intersection of this line with the edges of the quadrilateral. This is illustrated in Figure 4.3-2 b.

A perturbed triangular lattice can be formed using a method similar to that described for the square lattice and the technique is illustrated in Figures 4.3-3 a, b, and c. First a regular triangular lattice is produced. Each site, $i$, of this lattice is perturbed by an amount $r_{p, i}, \theta_{i}$ as discussed above and shown in Figure 4.3-3 a. The perturbed sites are then connected to form a mosaic of irregular triangles and the centroids of these triangles are found as shown in Figure 4.3-3 b. These new centroids become the vertices of a mosaic of irregular hexagons that cover the region as shown in Figure 4.3-3 c. The centroids of these hexagons then become the new sites. Once again, crack orientation is determined from the local principal continuum stress at the new site and a line with this orientation is drawn through the new site. The intersection of this line with the edges of the perturbed hexagon determines the crack length.

\subsubsection{Site Density}

Both the crack length and crack density depend on the lattice type. This is due to the difference in site density. For a given lattice constant $l_{c}$, triangular and square lattices have much different values of crack density. The difference in crack density of these lattices affects the number of cracks. Maximum crack densities for square and triangular lattices are 1 and 1.15, respectively. Crack lengths for unperturbed triangular and square lattices were $0.8 l_{c}$ and $0.9 l_{c^{\prime}}$ respectively. Crack lengths for sites cracked on irregular lattices were determined on a site-bysite basis as discussed in the previous section.

\subsubsection{Crack Linking}

The concept of the field-theory model is to simulate cracking by inserting displacement discontinuity elements at cracked sites and orient the elements with respect to the principal continuum stress at the site (Section 4.1.4). However, the treatment of cracks at neighboring sites, especially those ahead of the crack tips, must be considered. In a preliminary evaluation, displacement discontinuity elements were inserted as independent entities with no explicit 


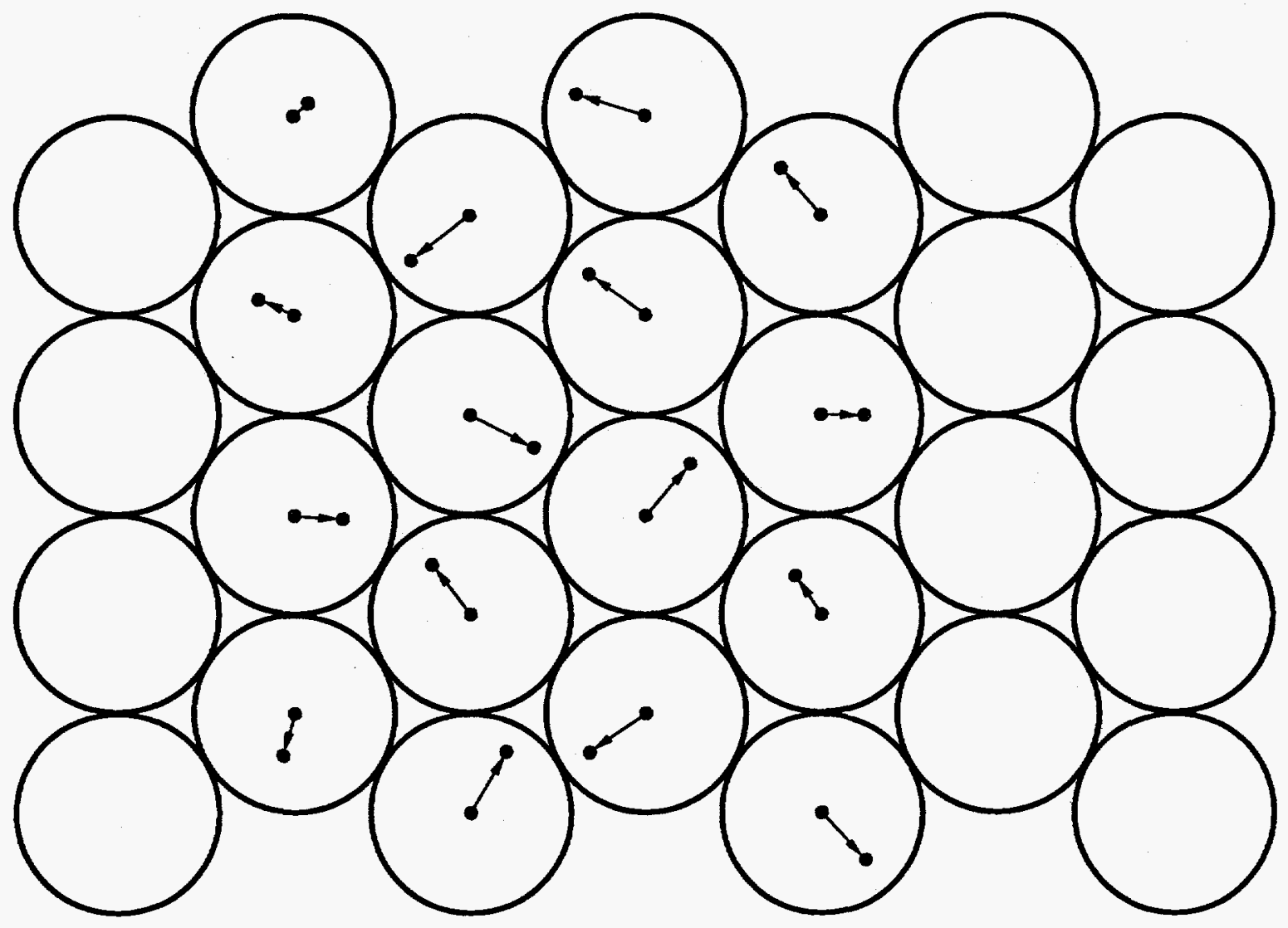

Figure 4.3-3. Production of perturbed triangular lattices: a) original sites are relocated to new coordinates. 


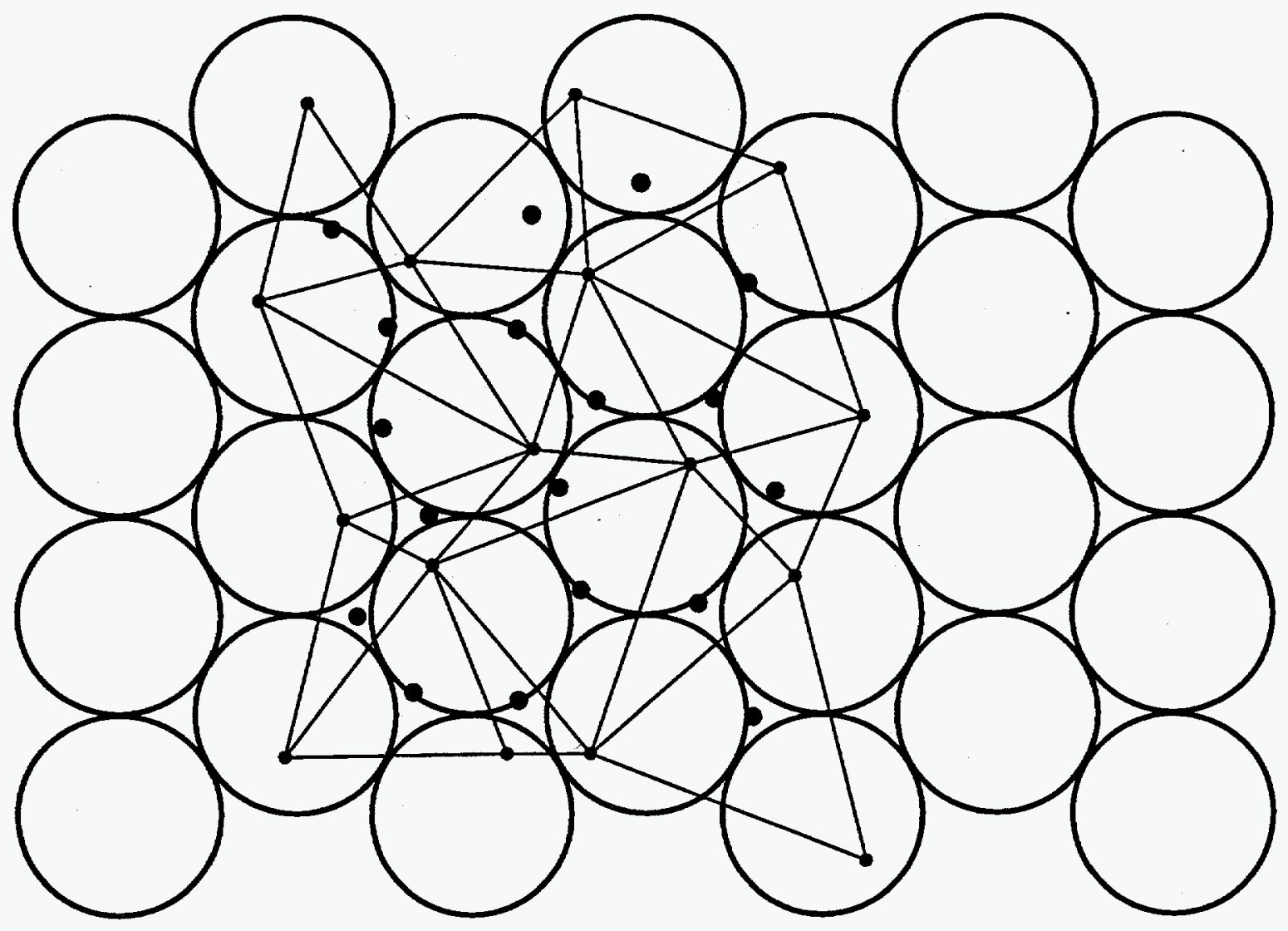

Figure 4.3-3b. Sites at new coordinates are connected to form a new triangular grid. Center points of triangles on the new grid are located. 


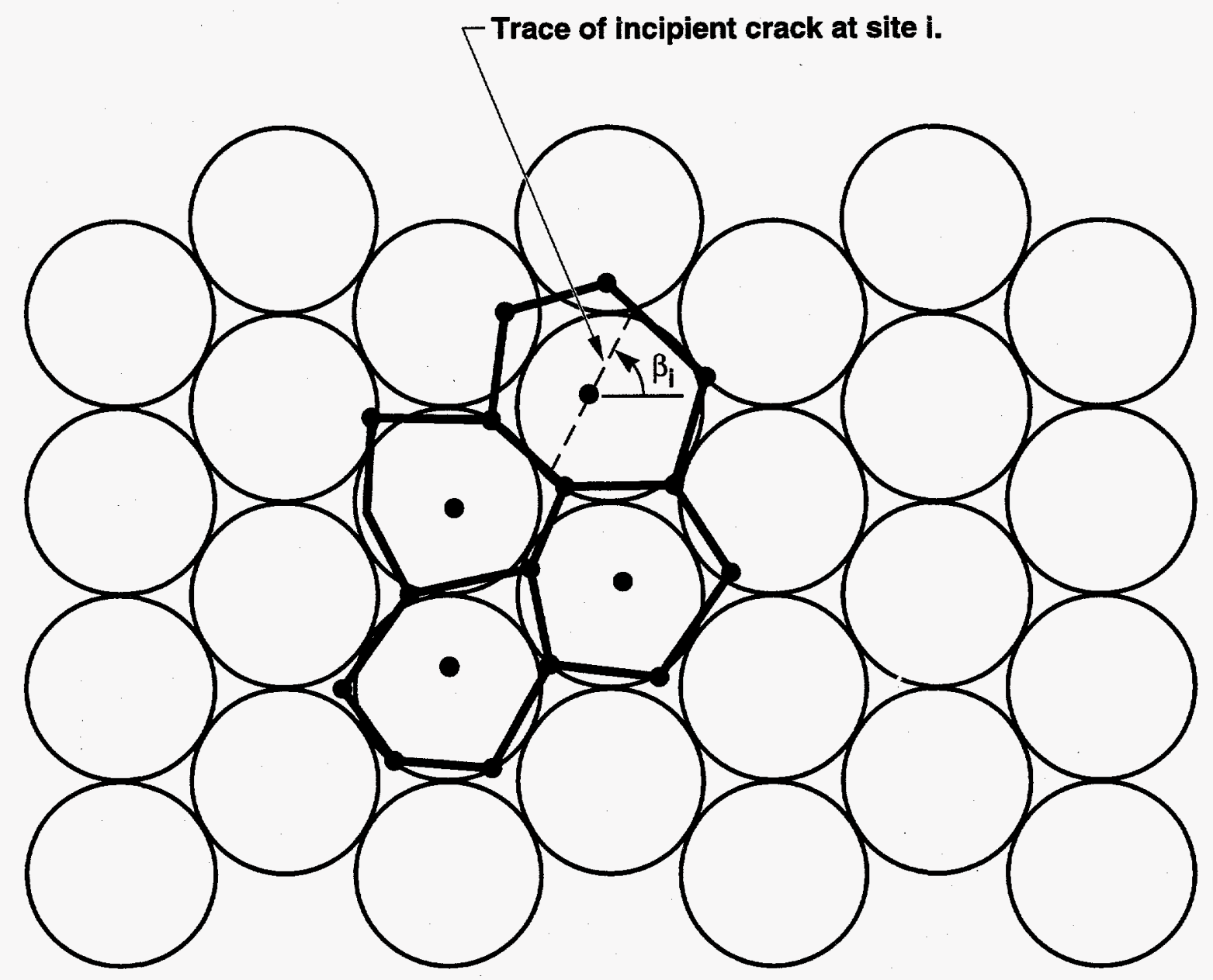

Figure 4.3-3c. Center points are connected to form irregular hexagons. The center of mass of each new hexagon becomes a site on the perturbed lattice. 
linking between elements forming neighboring cracks. The results of this implementation were unsatisfactory, as the breaking of several neighboring sites often caused numerical instability in the model associated with the stress singularity that occurs at each crack tip. This method was also physically unrealistic given extensive experimental and field evidence for crack linking. In real materials en-echelon cracks coalesce if formed in close proximity, because of the high stress concentrations at the tips.

Consequently, a linking methodology was developed to allow boundary elements at neighboring sites to connect if certain criteria are met. A crack is formed at a site $i$ by insertion of three boundary elements, as discussed in Section 4.1.4. The crack is assigned an orientation index based on the value of $\beta$, as shown in Table 4.3-1. The crack at $i$ is connected to a crack at a neighboring site $j$ if the crack at $j$ also has the same orientation index. This algorithm is illustrated in Figure 4.3-4. The internal tractions for the boundary elements forming the connecting crack are set to zero, making this a stress-free surface, to preserve equilibrium. The insertion of the connecting crack does not affect the percolation behavior as the connecting crack is considered as part of the crack at the new site.

This completes the discussion of techniques used to implement the model in a numerical code. Code was written in $\mathrm{C}$ and components are described in Section 4.5. Details of percolation algorithms and other algorithms are given by Daveler and Blair (1994). The next section describes how the code was configured to simulate lab tests.

Table 4.3-1. Crack linking.

\begin{tabular}{cc}
\hline Crack orientation & Linking index \\
\hline$-\pi / 6 \leq \beta<\pi / 6$ & 1 \\
$5 \pi / 6 \leq \beta<7 \pi / 6$ & \\
$\pi / 6 \leq \beta<\pi / 2$ & 2 \\
$7 \pi / 6 \leq \beta<3 \pi / 2$ & \\
$\pi / 2 \leq \beta<5 \pi / 6$ & 3 \\
$3 \pi / 2 \leq \beta<11 \pi / 6$ & \\
\hline
\end{tabular}




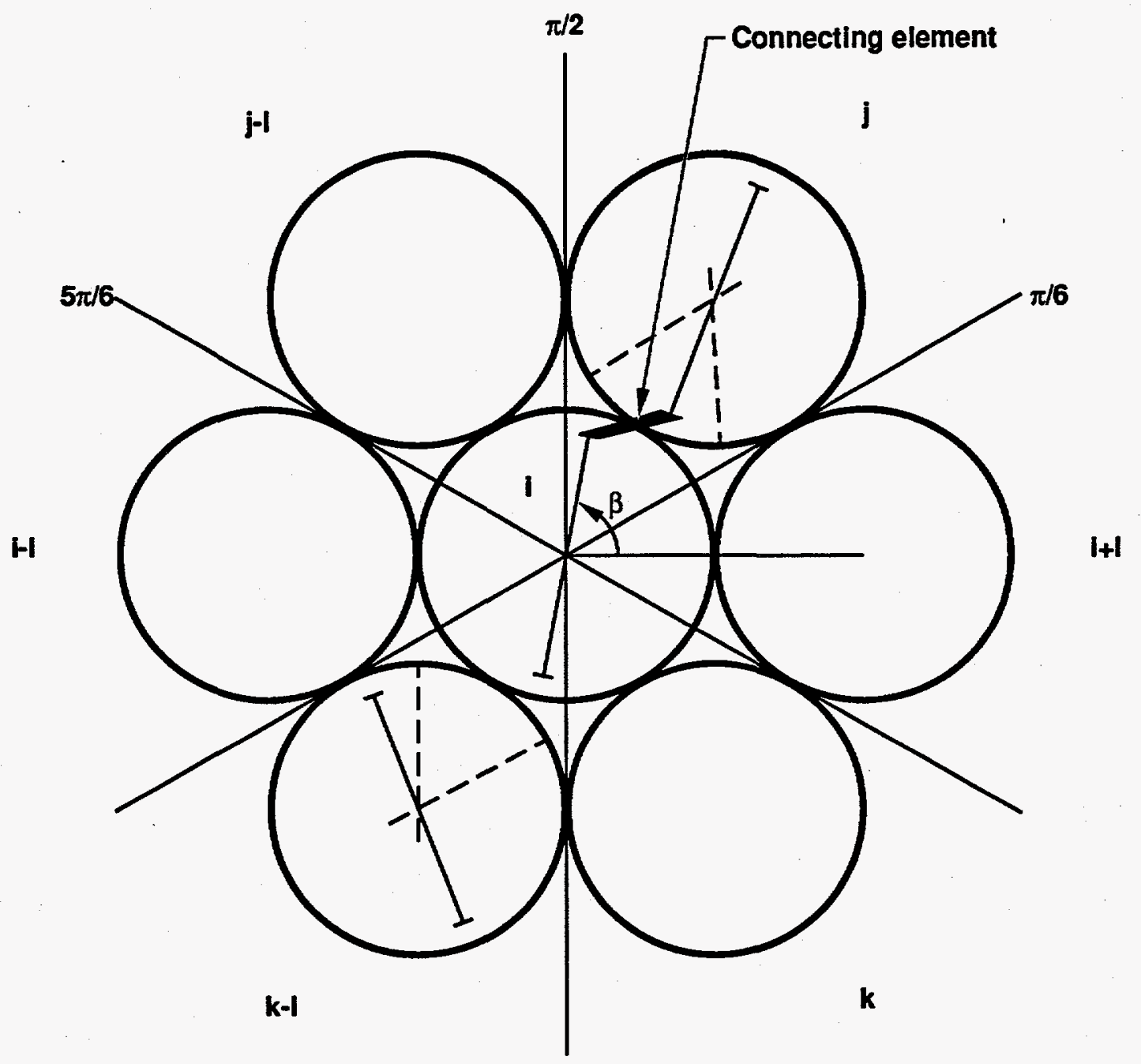

Figure 4.3-4. Crack linking on a triangular lattice. 


\subsection{Simulation of Compression Tests}

To demonstrate the behavior of the field-theory model and to assess how it simulates the deformation of rock in compression, the model was configured to simulate laboratory uniaxial and triaxial compression tests in two dimensions. These tests were chosen because they are widely used in the rock mechanics community to characterize rock behavior, and standard procedures have been developed (American Society for Testing and Materials). Moreover, numerous detailed studies have been done using this geometry, including the classic studies of Wawersik and Fairhurst (1970) and many others. More recently Lockner et al. (1991) have experimentally mapped crack propagation by locating acoustic emissions during compression tests. Consequently, there are extensive data that can be compared with the prediction of the model.

It is important to note that in the field-theory simulations no attempt is made to incorporate kinematic stability of the simulated sample. Moreover, laboratory studies have shown that much of the post-peak strain-softening observed in laboratory compression tests is due to kinematic behavior, such as slabbing of the sample and sliding along inclined fractures. The stress-strain behavior predicted by the field-theory model is also affected by other assumptions made in the model and by the way the model is operated to simulate a test. Details of the stress-strain simulations are discussed below.

As discussed previously, the concept of the model is to discretize the region of interest and use BEM to handle complex geometries and regions of continuum stresses outside of the discretized region. A test geometry as shown in Figure 4.4-1 was used to simulate uniaxial compression tests. This geometry satisfies many of the criteria for a triaxial test, as given in the ASTM procedure. In particular the length-to-width ratio of the discretized region is 3 to 1 . The ASTM procedure recommends that samples be tested with length-to-diameter of 3 to 1 and with a minimum of 2.5 to 1 . Array height is given by ISZ; array width is given by JSZ. 


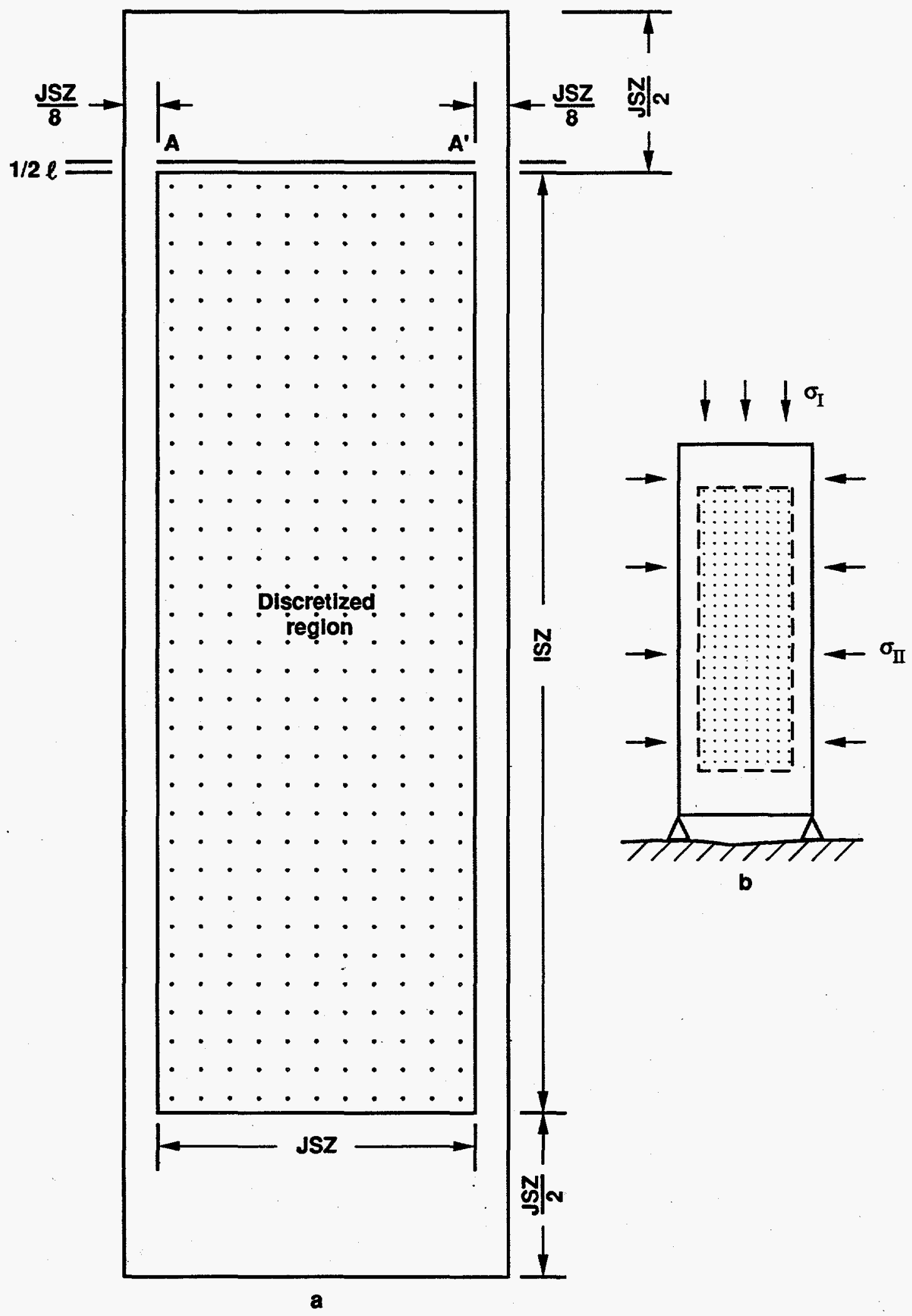

Figure 4.4-1. Schematic of geometry used in simulated compression tests. a) relative array dimensions b) boundary conditions 


\subsubsection{Computation of Macroscopic Stress-Strain Behavior}

This section describes how macroscopic stress-strain curves are determined for the simulated laboratory compression tests. The axial stress for the region $R$ in simulated compression tests is computed along the line $\mathrm{A}-\mathrm{A}^{\prime}$ in Figure 4.4-1. This location was chosen to provide a measure of the stress in the region where cracking can occur, and to reflect the decrease in stress in that region that occurs when cracks form.

Axial stress along line A-A' in $R$ is computed as follows. The stress in $R$ at a series of sites in the $y$ direction along $A-A^{\prime}$ is computed via superposition of stress in the $y$ direction for the equivalent sites in $E$ and $S$. The axial stress is then computed as the average stress along this line in the $y$ direction using the expression

$$
\left(\sigma_{1}\right)_{R}=\frac{1}{m} \sum_{i=1}^{m}\left(\sigma_{y y, i}\right)_{E}+\left(\sigma_{y y, i}\right)_{S}
$$

summing over $m$ sites along A-A'.

The axial strain in $R$ is determined using the strain energy for $E$ and $S$ as discussed in Section 4.1 .8 , i.e.,

$$
\varepsilon_{R}=\frac{2 U_{R}}{\sigma_{1_{R}}}
$$

and

$$
U_{R}=U_{E}+U_{S}
$$

The component of strain energy due to the elastic response of $E$ is computed as

$$
U_{E}=\frac{\left(\sigma_{1 E}\right)^{2}}{2 E_{m}}-\frac{\sigma_{2} \varepsilon_{2}}{2},
$$

where

$$
\sigma_{1_{E}}=\frac{1}{m} \sum\left(\sigma_{y y, i}\right)_{E} .
$$

Strain energy for $S$ is calculated as

$$
U_{S}=\frac{1}{2} A \sum \rho_{i} l_{i} \delta_{i},
$$


where $A$ is the area of the simulated sample. Note that this assumes that all strain energy due to crack opening is put into axial strain. This conservative calculation is used because the simple displacement discontinuity element used in the model has no mechanism for producing an axial strain when a crack is opened in the lateral direction. Recall that all cracks are oriented parallel to the maximum local principal stress and are opened in the direction of the minimum local principal stress; thus in a uniaxial compression test crack opening will not produce axial strain. Other more complicated models have been developed for extensile cracks in compression, such as the sliding crack, that do provide for axial shortening. However, these require that more assumptions be made about the material behavior. Incorporating more assumptions would then require that the effects of these assumptions be identified, and separated from the effects of the heterogeneity that are the real focus of this study.

\subsubsection{Borders}

To simplify the calculation, cracks or fractures (clusters of cracks) are not allowed to connect to the external surface. Consequently, the discretized region to be studied is surrounded by a border of uncracked elastic material, and this constrains the model to be kinematically stable. In addition, the properties of this border region do not vary as cracks are added to the model. Ideally the border would be located as close as possible to the discretized region, to minimize the area of uncracked elastic material. However, in practice it was determined that both the numerical BEM method and the capability of computing resources limited the dimensions of the border region as follows.

\section{Top and Bottom}

The dimensions of the top and bottom borders are one-half of the width of the discretized array (see Figure 4.4-1). This relatively wide border is needed because the formulation of BEM used in the model does not allow propagation of a crack or fracture close to a free surface. Testing of the model configured to represent an isolated body showed that locating a long crack both close to and perpendicular to a free surface causes numerical instability in the BEM code. In 
this application of the BEM it was determined by trial and error that a top and bottom border of $\mathrm{JSZ} / 2$ was sufficient to keep the solution stable for long cracks in the solid region, where JSZ is the number of rows of sites on the lattice.

The thickness of the top and bottom borders was not considered a limitation in this study, because the material in this region is under direct compression and simply transfers force. Moreover, it is common practice in laboratory tests to use endcaps that are matched to the sample stiffness. The top and bottom border regions are analogous to matched endcaps.

\section{Side}

The width of the side borders is important because the material in each of the side borders remains uncracked and acts as an elastic pillar placed in parallel with the discretized region where cracks can occur. As the discretized region becomes more and more cracked, more and more stress is transferred to these pillars. The side borders also serve to confine the simulated sample. To minimize these effects, the side borders should be made as narrow as possible, and ideally the side borders would have zero width, support none of the stress, and provide no confinement. Moreover, if computational resources were infinite, the ideal situation could be approached by making the length of elements forming the cracks and the borders arbitrarily small. This would work as long as the cracks inserted in the discretized region were more or less parallel to the border. Given the computational resources available for this study, it was necessary to determine an acceptable width for the side borders. This served to reduce the total number of boundary elements to a number that could be computed on available workstations and to shorten the time necessary to complete a trial.

Several border widths were evaluated. The narrowest width considered was JSZ/16 and a few trials were attempted with this side border arrangement, and for array sizes of $10 \times 30$ the computational time per trial was more than 24 hours. For array sizes of $20 \times 60$ or greater, trials using this narrow border ran for more than one week and did not finish due to memory limitations. 
The border width of JSZ/ 8 was also evaluated. This required fewer elements and reduced both the memory requirements and the run times. Stress-strain behavior for $20 \times 60$ arrays computed using these two side border conditions are compared in Figure 4.4-2. This figure shows that for most of the stress-strain curve, results are identical. The behavior is different only near the peak. The peak stress for the smaller side elements case is $\sim 1 \mathrm{MPa}$ lower than for the case with longer elements, and less strain is observed. Moreover, the behavior is very similar and because the trials with border width of JSZ/8 (2.5 elements for a $20 \times 60$ array) ran considerably faster, this width was chosen for the side border. The use of a wider boundary does increase the predicted stress at a given strain, but the effect is small.

\subsubsection{Boundary Conditions and Loading Path}

To simulate laboratory compression tests, the boundary conditions shown in Table 4.4-1 and Figure 4.4-1 were used.

Table 4.4-1. Boundary conditions for simulation of compression tests.

\begin{tabular}{llll}
\hline Location & Type & Normal & Shear \\
\hline Top & Mixed & Stress: $\sigma_{\mathrm{n}}=\sigma_{\mathrm{I}}$ & Displacement: $u_{\mathrm{s}}=0$ \\
Bottom & Displacement & Displacement: $u_{\mathrm{n}}=0$ & Displacement: $u_{\mathrm{s}}=0$ \\
Sides & Stress & Stress: $\sigma_{\mathrm{n}}=\sigma_{\mathrm{II}}$ & Stress: $\sigma_{\mathrm{s}}=0$ \\
\hline
\end{tabular}

Axial stress was applied in steps, and sites were broken sequentially as described in the following section. This loading path is similar to using a soft loading machine. That is, a stress level is applied and is maintained until cracking stops or a percolating cluster is formed. This loading path is useful for examining how different types of grain-scale heterogeneities affect the evolution of crack systems and, until kinematic instability or unstable crack growth occurs, the loading is similar to that done in a standard test where only stable conditions are imposed. Moreover, recently Tang (1994) has argued that fracture of rocks in the natural environment, such as during earthquakes and volcanic eruptions, occurs under unstable loading conditions and the 


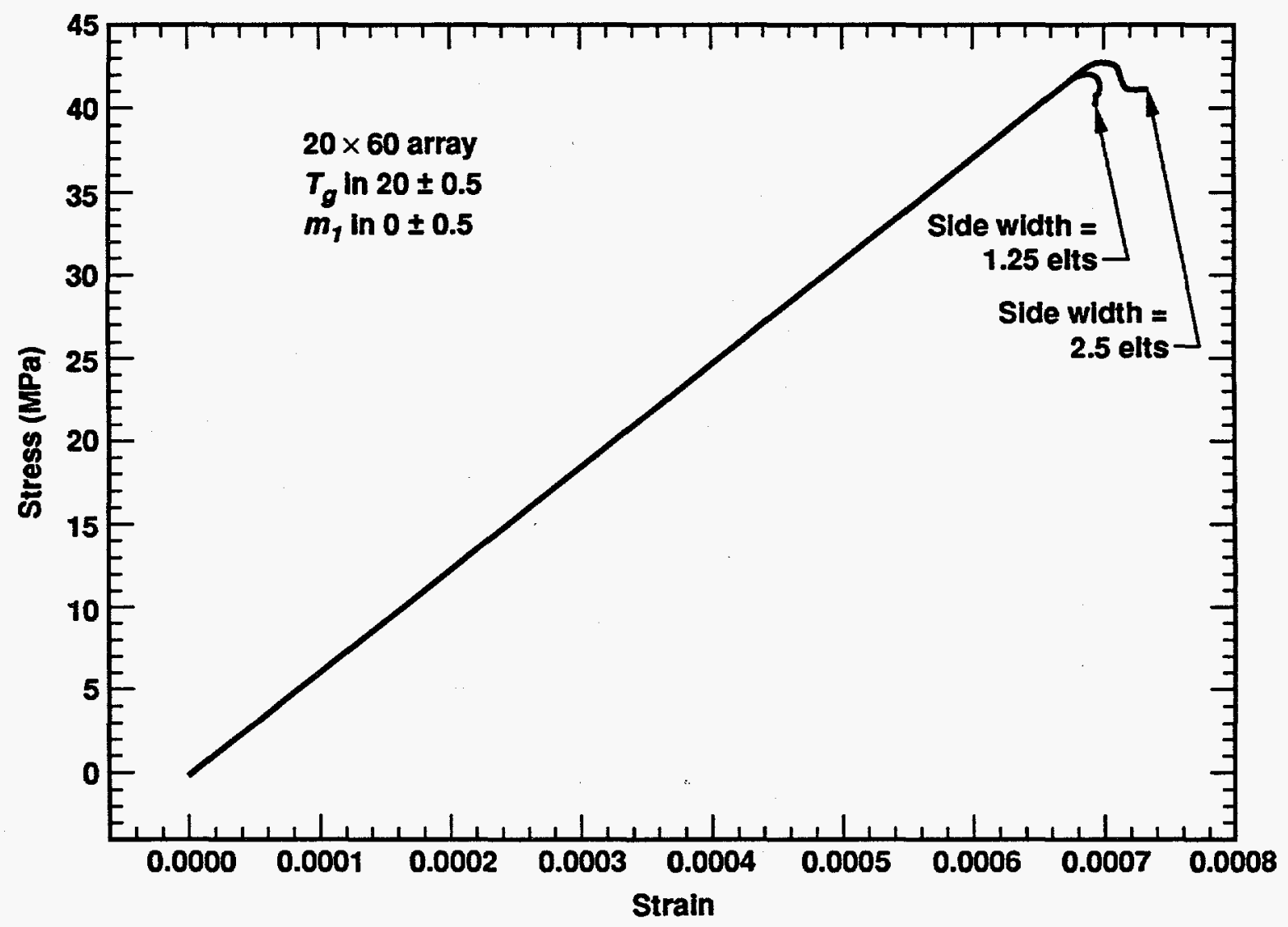

Figure 4.4-2. Effect of side border width on stress-straln behavior. 
failure process under these conditions may be different from that observed in servo-controlled tests. The field-theory model can be used to simulated a variety of loading paths, but this involves additional programming and testing, and is left for future work as discussed in Chapter 7.

\subsubsection{Failure Criterion}

\section{Implementation of Breaking Rule}

To simulate laboratory compression tests, an increment in axial stress was applied and the stress fields in $E$ and $S$ were computed. $\sigma_{B, i}$ was computed following equation 4.9 and compared to $T_{g, i}$ for all sites. A site qualified to break if the condition expressed in equation 4.13 was met. If no sites qualified to break, another stress increment was applied. If more than one site qualified then the one with the highest overstress ratio, 1

$$
Q_{i}=\frac{\sigma_{B, i}}{T_{g, i}}
$$

was broken and a crack was inserted at that site. The array was then checked for the existence of a percolating cluster, and if such a cluster was found, the stress field was recomputed to reflect the final state of stress, and the simulation was stopped (a percolating or critical cluster is one that spans the lattice from top to bottom). If a percolating cluster was not found, the stress field was recomputed and the breaking rule was evaluated again. This cycle continued at a given level of applied stress until no more sites broke or a percolating cluster of cracks was formed. If no more sites broke and a percolating cluster had not formed, another stress increment was applied. The simulation stopped when percolation occurred. This method provided the capability for crack growth at a constant stress level.

1 de Arcangelis (1990) studied cracking in a lattice beam model with random site strength distributions and used the overstress ratio plot to describe three regimes of fracture: an initial regime where cracks form randomly and their locations are controlled by the disorder in site strength, a regime of stable crack growth where the stress field controls breaking, and a catastrophic regime of unstable crack propagation where the system fails and overstress values can fluctuate widely. 
The peak strength for a trial is determined by the way the model is operated. For simulating laboratory compression tests, the axial stress is increased a step at a time, and if a site breaks a crack is inserted, stresses and strains are recalculated, and clusters of cracks are identified and tested to determine if a critical cluster has formed. If a critical cluster was present, the array was said to fail and the trial was stopped at that point. If no cluster spanned the lattice, the loop continued until no more sites broke, then the axial stress was increased. As explained earlier, the model was formulated with a narrow strip of elastic material along the sides of the array, and insertion of a crack generally caused stress to be transferred from the discretized region into these elastic strips, with a corresponding decrease in the stress calculated along line A-A' in Figure 4.4-1. An increase in stress level applied at the boundary caused an initial increase in the stress at A-A', but as cracks formed stress was transferred to the uncracked material in the side borders, decreasing the stress and increasing strain in the cracked region. Consequently, the stress-strain curves consist of a series of peaks followed by strain softening.

Using the percolation criterion as formulated in the nearest-neighbor model (Figure 3.1-3), a critical cluster was formed and the trial was terminated when a cluster met one of the seven failure criteria. In practice, it was determined that these criteria were not satisfactory. This is because tracing of the failure path shows that for many of the trials, failure occurred along a path from the top to the side (e.g., paths A-C, A-E, D-B, D-F in Figure 3.1-3). Simulations done using the field-theory model showed that these crack patterns often form a long narrow chip or slab that is analogous to slabbing, which is often observed in laboratory tests. While prediction of this type of slabbing is a validation that the model simulates behavior often observed in rock, it is important to note that in laboratory tests, these slabs generally form before the peak stress is reached. Given the failure criteria for Figure 3.1-3 the trial would be stopped and the peak strength would be more indicative of the strength at which chips formed.

Additional insight on the failure criteria used in Chapter 3 can also be gained by inspection of Figure 4.4-3. In this figure the critical cluster meets one of the criteria for fracture, as it spans sides B-D. However, the path is quite tortuous, and a rock with a connected set of cracks in this 


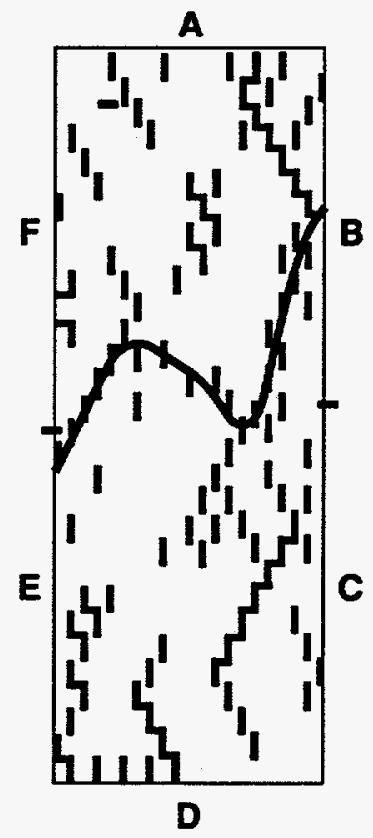

Figure 4.4-3. A cluster spans the lattice from $B$ to $E$, but does not satisfy kinematic failure criterion. 
geometry would retain considerable compressive strength. In fact, a crack with this geometry would have a very small effect on the rock compressive strength.

Upon review of the above results, it became apparent that the percolation criteria used in Chapter 3 are inadequate. The results from several preliminary studies showed that using a failure criterion of top-to-bottom percolation gave the most consistent measure of model behavior. Consequently, a top-to-bottom percolation criterion was adapted as the failure criterion in the work presented subsequently.

\subsubsection{Discussion}

The stress-strain curves predicted in this study reflect several assumptions made in the fieldtheory model and the way that the model is operated. The intent of simulating stress-strain behavior is to study the effect of the formation of microcracks and crack interaction on macroscopic behavior in a system with microscale heterogeneity. No attempt is made to incorporate kinematic failure and the loading path is that typical for a soft loading machine. Moreover, all strain energy from crack opening is converted to axial strain, and the calculation stops when a cluster of cracked sites percolates from the top to the bottom of the array. Given these assumptions and methods, it is expected that the stress-strain curves will overestimate strain at elevated stress levels in the post-peak region of the curve, as the material is not allowed to fall away and the axial stress is held constant. The stress-strain curves do provide insight into the importance of microscale heterogeneity, formation of damage, and crack interaction in the deformation and failure processes that occur when rock is subjected to compressive stress.

This model can easily be used to study the fracture of materials under a variety of load paths and variation of load path by varying boundary conditions is generally straightforward. However, a load path that was not tried was servo-controlled loading and/or unloading. To simulate this type of loading the axial load for a compression test would be increased until one site cracked. A crack would be inserted at that site and the axial load would be reduced below the level at which any site breaks and then incrementally increased until the next site breaks. 
This would be useful for simulating the servo-controlled compression tests used by Lockner et al. (1991) to study stable crack propagation (see Section 6.2).

\section{Strain Rates}

The effect of loading rate is important in many engineering applications such as hydrofracture, delay blasting, and loads due to impact. Rate implies a time dependence and the field-theory model is currently static, i.e., has no time dependence. One of the assumptions often made about material behavior is that loading is slow in comparison with the stress redistribution of stress in the material and, as discussed earlier, the calculations performed for this thesis make this assumption. While this assumption is valid for many situations, there are several applications where loading rates fall outside the range where it is valid such as impact loads. In the case of intermediate strain rates the loading rate is roughly the same as the rate of stress redistribution. In this case the stress does not have time to become redistributed. The model can easily be adapted to simulate more rapid loading. To do this the "one at a time" breaking rule would be altered so that all sites with overstress ratio $>1$ are broken simultaneously, and a crack is inserted at each broken site. This would result in an immediate population of small cracks. The stress field for the new crack distribution would then be recomputed and the breaking rule evaluated and again, all sites that qualify would be broken simultaneously. This type of breaking rule can easily be implemented in this model and may produce results that are quite different from those reported in this thesis. A simple variation on this would be to break only a fraction of the sites that qualify (say, 0.1 or 0.5 of the qualifying sites).

The model could also be adapted to simulate very slow processes such as subcritical crack growth that occur at rates much lower than usually considered. Subcritical crack growth refers to extension of a crack when the stress intensity factor is less than the critical stress intensity factor necessary for crack extension. This could be incorporated into the model by assigning timers to sites in high stress regions and incrementally decreasing the strength at these sites. This could be useful for the study of mine pillar design and/or well bore stability. 


\subsection{Numerical Implementation}

The theoretical development of the field-theory model presented in this chapter has been implemented in a suite of numerical computer codes or programs written in the $C$ language. Each of these programs is in turn composed of a series of subroutines. The model is named FRACROCK; the system of codes comprising the model is listed in Table 4.5-1 and illustrated in Figure 4.5-1. The code development has been controlled using the source code control system (sccs) that is available on most UNIX operating systems. These codes use the $\mathrm{X}$-windows display system and interactive graphics. Values for the stochastic arrays are produced by the Splus (Becker et al., 1988) language. This section briefly discusses the individual programs that make up FRACROCK, and is intended to document the style in which the component programs are designed and used. The model is documented in a report by Daveler and Blair (1994).

The primary code in the model is called astool. Two versions of this code have been produced. astool is an interactive code that is used to set up individual problems and to demonstrate or evaluate the code in different configurations. astool_batch is identical to astool but has the interactive graphics and display capabilities disabled. This code is used to run a sequence of trials at a particular condition. Other auxiliary codes that are used to display and reduce the output of astool are described below.

\subsubsection{Programs astool and astool_batch}

Function. This is the primary computational tool in the FRACROCK model. This program performs the following functions.

Setup of problem. This program is used to generate the input for the problem to be computed. This includes definition of the size, shape and type of grid; specification of the stochastic distributions for site strength and site shape factor; definition of excavations in the region, specification of loading path and all boundary conditions; and selection of stress component to be recorded for later viewing using the movie program (see below). 
Table 4.5 - 1 Codes and functions used in Fracrock model

\begin{tabular}{|llll|}
\hline Code Name & Function & Input files & Output files \\
\hline Fracrock & Model & set-parameters.dat & $\begin{array}{l}\text { sequence.crk } \\
\text { movie files }\end{array}$ \\
Fracrock_batch & Model & set-parameters.dat & $\begin{array}{l}\text { sequence.crk } \\
\text { movie files }\end{array}$ \\
Fractool & Analysis & sequence.crk & PostScript files \\
Moviool & Analysis & sequence.crk & PostScript files \\
& Analysis & movie file & $\begin{array}{l}\text { PostScript files } \\
\text { HDF image files }\end{array}$ \\
\hline
\end{tabular}

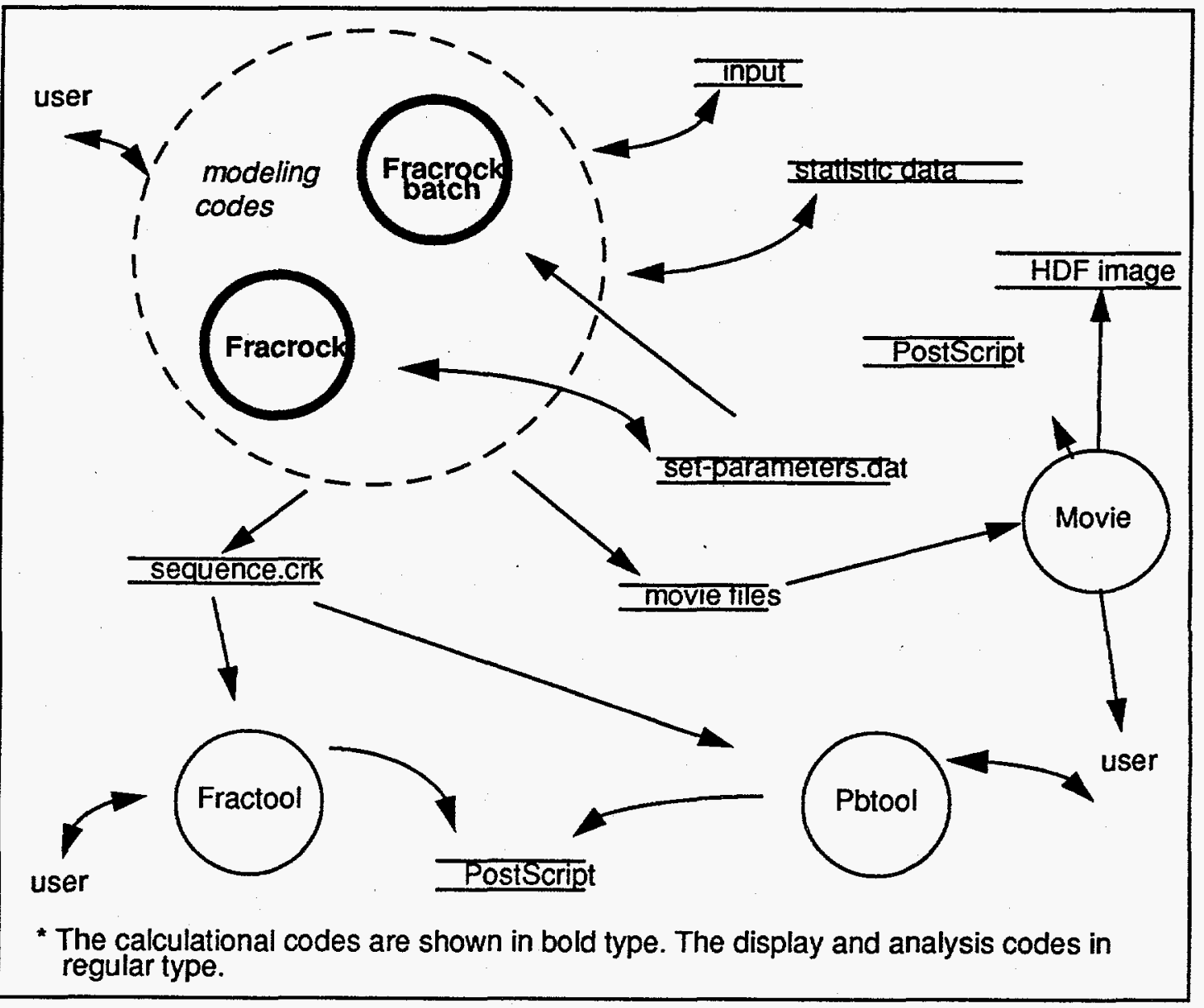

Figure 4.5 - 1 Fracrock system of codes and files 
Execution of field theory logic. astool also contains the boundary element method routines for the displacement discontinuity method as presented in TWODD (Crouch and Starfield, 1990) for computation of the stress and deformation fields, routines for evaluation of the breaking rule, insertion of cracks, computation of strain, and evaluation of fracture criterion. The logic of the calculation is shown in Figure 4.5-2.

Creation of primary data files. This program produces a variety of data files that are used by this and other programs. These output files include:

- set-parameters.dat, which contains all information needed to describe the problem setup,

- sequence.crk, which records the sequence of cracking including the applied stress levels, index of cracked sites, and stress and strain values computed after each cracking event, and

- movie output file (optional), which contains an index of all broken sites and stress levels of all unbroken sites at each stage of the cracking process.

astool also has a sophisticated graphics display capability that provides a user-friendly interface for setup of the problem, and high-quality display of selected stress and displacement fields, as well as location of broken sites as the problem progresses.

astool_batch is identical to astool, but is configured to execute repeated trials in batch mode. Thus in astool_batch the graphics display capabilities have been removed. astool_batch requires input file set-parameters.dat and (optionally) files for site strength and stress diffenence multiplier $\left(m_{1}\right)$ distributions.

\subsection{2 fractool}

Function. This is a data analysis and display program that implements analytical routines that operate on the output files from astool and astool_batch.

Input. Sequence.crk file described above.

Output. This is the main plotting program for such output as stress-strain, broken sites at fracture, overstress vs number of sites broken, strain energy vs number broken. This program also produces a table that gives percolation threshold, ultimate stress, strain at ultimate stress, 
Initialize program variables. Read the file set-parameters.dat. Get statistical data

MAIN LOOP

Increment step counter. If step counter is greater than the number of steps to model

END TRIAL

Otherwise

Increment vertical stress

Compute stresses for elastic lattice using boundary element method

INNER LOOP

Calculate stresses for crack interaction lattice using boundary element method

Compute local tension using elastic and crack interaction stresses and $m_{1}$ values

Anneal $\mathbf{m}_{1}$ disorder

Compare tension at each site with tensile strength.

If tensile strength of all sites is greater than the associated tension (i.e. no sites break)

Go to the MAINLOOP

Otherwise

Find the index of the site with the largest difference between tensile strength and tension, where tension is greater than tensile strength.

Mark site as cracked, and update the lattice clusters (all adjoining sites form a cluster)

If any cluster connects the top of the lattice to the bottom

LATTICE IS BROKEN, END TRIAL

Otherwise

Add boundary elements, which describe the new crack, to the input file for the crack interaction lattice.

Go to the beginning of the INNER LOOP

END INNER LOOP

END MAIN LOOP

Figure 4.5 - 2. Fracrock program outline for rock mechanics calculations 
and stress at percolation. Many of the plots used in this thesis were produced using this program, and data contained in the tables were extracted from the sequence files for trials using this program. This program allows side-by-side comparison and/or averaging of results from several trials.

\subsection{3 movie}

function. This program provides a facility for graphical playback of the computed stress fields and sequence of breaking of a given trial. It also provides for simultaneous plotting of stress-strain behavior and cumulative strain energy due to cracking, while the breaking sequence and stress fields are being displayed. The program provides high-quality color display of the stress field and is useful for qualitative analysis of the evolution of the fracture, and for troubleshooting of the algorithms incorporated in the model. This program provides a very effective display of the progressive fracture predicted by the field-theory model.

input. $\mathrm{msig}^{* * *}$ file from astool or astool_batch, sequence.crk.

output. Dynamic onscreen display of stress fields and broken sites, single images of stress field and broken sites in a format compatible with transfer to video tape.

\subsection{4 pbtool}

function. To recompute stress fields at any point in a trial previously computed by astool or astool_batch. This program allows any stress or displacement component to be displayed at any point in a trial that has been completed. This program is useful for creating graphic display files and for detailed analysis of a particular trial.

input. Sequence.crk

output. Images of stress fields and location of broken cells, plot of number of clusters vs cluster size. 


\subsection{Results of Parameter-Sensitivity Analysis: Effect of microscale heterogeneity on macroscopic deformation}

The goals of this thesis are to explore the effect of microscale heterogeneity on macroscopic behavior in rocks, and to evaluate the role of crack interaction in rock deformation and fracture. This chapter presents results of a parameter-sensitivity analysis conducted to address the first of these goals. The purpose of the analysis is to determine how variation of several independent microscale parameters affects behavior in simulated compression tests. Independent parameters that were explored include: heterogeneity in local stress, site strength, type and size of lattice, and perturbation of lattice site location. The dependence of model behavior on these parameters is evaluated in terms of macroscopic stress-strain behavior, ultimate strength derived from the stress-strain curve, crack patterns, percolation threshold (i.e., crack density when a cluster spans the lattice), crack strain energy, and other related parameters.

One of the first tasks of the parameter-sensitivity analysis was to conduct a series of exploratory simulations to select a set of baseline conditions. Then the effect of each parameter on behavior was evaluated by holding all parameters at the baseline value and then varying the one of interest. The full range of variables and the baseline condition selected for each one are presented in Table 5.0-1. Most of the computations were carried out using a triangular lattice. This lattice was chosen because it is isotropic and commonly used in the statistical modeling community. Other input parameters that were not varied include Young's modulus and Poisson's ratio. These were held constant at values of $70 \mathrm{MPa}$ and 0.25 , respectively, for all trials as described in Section 4.1.7. Complete data describing all sets of trials computed for this analysis are presented in the Appendix. Most of the sets of trials were conducted on arrays with size of 10 columns by 30 rows (10×30), and generally 10 trials per set were conducted. Larger arrays, especially the $30 \times 90$ arrays, had fewer trials because of computational constraints on time and the size of computer available. 
Table 5.0-1. Summary of parameters used in sensitivity analysis.

\begin{tabular}{lll}
\hline \multicolumn{1}{c}{ Parameter } & \multicolumn{1}{c}{ Values } & \multicolumn{1}{c}{ Baseline } \\
\hline Site strength $\left(T_{g}\right)$ & Uniform over $20 \pm 0.2,20 \pm 19.9,26 \pm 0.26$, & Uniform over 20 \pm 19.9 \\
& $\begin{array}{l}35 \pm 34,50 \pm 0.5,50 \pm 49.5, \text { bimodal 2(25\%), } \\
26(75 \%)\end{array}$ & \\
& Uniform over $\pm 0.3, \pm 0.5, \pm 0.7, \pm 1.0$ & Uniform over \pm 0.7 \\
Local stress & & \\
heterogeneity & $10 \times 30,15 \times 45,20 \times 60,27 \times 81,30 \times 90^{\mathrm{a}}$ & 10×30 \\
Lattice size & Triangular, square, perturbed triangular, & Triangular \\
Lattice type & perturbed square & \\
\hline
\end{tabular}

${ }^{a}$ Number of columns $\times$ number of rows.

The baseline size of $10 \times 30$ was chosen after reviewing results of preliminary trials that showed that this size provided values that were good indicators for the behavior, and because a set of 10 trials of this size could be computed in approximately 24 hours. The effect of size is discussed in detail in Section 5.1. Sets of 20 to 30 trials did not noticeably improve the statistics. A loading path with $\Delta \sigma_{1}=2 \mathrm{MPa}$ was chosen because it is consistent with assumptions on rate effects (see Sections 4.4 .4 and 7.1.3) and consistent with procedures in laboratory compressive tests. Finally, uniaxial compression tests were simulated in this study so a confining pressure of $0.1 \mathrm{MPa}$ (atmospheric or unconfined) was used. The rationale for the choice of baseline conditions for the local stress heterogeneity (i.e., $m_{1}$, the stress difference multiplier) is presented in Section 4.1.3, and that for the site strength parameter $T_{g}$ is presented in Section 5.3. In the following sections the results of the parameter-sensitivity study are systematically discussed.

This chapter is organized as follows. Section 5.1 presents the results of studies of how lattice size affects macroscopic behavior. Sections 5.2 and 5.3 present results of studies of how heterogeneity in distributions of local stress heterogeneity and site strength, respectively, affects macroscopic behavior for trials computed using triangular lattices. Section 5.4 examines 
the effect on macroscopic behavior of perturbing lattice site locations on a triangular lattice. Sections 5.5 and 5.6 present results of studies of how heterogeneity in distributions of site strength $\left(T_{g}\right)$ and site geometry $\left(m_{1}\right)$, respectively, affects macroscopic behavior for trials computed using square lattices. Section 5.7 examines the effect on macroscopic behavior of perturbing lattice site locations on a square lattice. Section 5.8 presents a summary and conclusions for the parameter-sensitivity analysis.

\subsection{Effect of Lattice Size}

The purpose of this section is to show how lattice size affects the macroscopic behavior predicted by the field-theory model. To study the effect of lattice size on macroscopic behavior, four different lattice sizes were used: $10 \times 30,20 \times 60,27 \times 81$, and $30 \times 90$. These correspond to lattices with $300,1200,2187$, and 2700 sites, respectively. Trials used for assessment of lattice size were computed with strength and geometric parameters set at baseline conditions $T_{g} 20 \pm 19.9 \mathrm{MPa}$ and $m_{1}$ in \pm 0.7 , respectively. These ranges were chosen because they represent the properties of a sandstone with a moderate amount of heterogeneity, such as Berea sandstone.

The range of lattice sizes spans approximately one order of magnitude and, while it was desirable to use larger arrays, the structure of the numerical code is such that large amounts of random access memory (RAM) are required for the boundary element calculations. The $30 \times 90$ size was the largest size that would run to completion on a computer with 256 megabytes (MB) of RAM without restructuring the code, and this was the largest computer capability available at reasonable cost for the work.

The general effect of variation in lattice size is presented in Section 5.1.1, which discusses the behavior of four single trials, one computed at each of the four sizes listed above. Summary data for sets of several trials are presented in Section 5.1.2. These data describe the dependence of average properties such as mean ultimate strength and mean percolation threshold on the lattice size. 


\subsubsection{Single Trials}

This section presents results of four single trials, one computed at each of the four sizes, $10 \times 30,20 \times 60,27 \times 81$, and $30 \times 90$. Summary data for these trials are presented in Table 5.1-1.

Table 5.1-1. Summary data for single trials.

\begin{tabular}{ccccc}
\hline Trial & Lattice & Size & $\sigma_{\text {pk }}$ & $p_{c}$ \\
\hline seq10.8_26_94.9 & $10 \times 30$ & 300 & 29.0 & 0.26 \\
seq20.8_26_94.9 & $20 \times 60$ & 1200 & 27.4 & 0.29 \\
seq27.4_19_94.3 & $27 \times 81$ & 2187 & 23.3 & 0.23 \\
seq30.8.x1 & $30 \times 90$ & 2700 & 16.9 & 0.18 \\
\hline
\end{tabular}

Results for these trials are presented in terms of stress-strain, normalized crack strain energy, and crack patterns.

\section{Stress-Strain}

Stress-strain curves for each of the four trials are shown in Figure 5.1-1. It is important to note that the values of stress and strain shown are those computed after each cracking event and are plotted sequentially. ${ }^{1}$ For these trials we observe that nonlinearity begins at applied $\sigma_{\mathrm{I}}$ values as low as $7 \mathrm{MPa}$, and significant nonlinearity occurs at axial stresses greater than $15 \mathrm{MPa}$. Nonlinear behavior is shown after the peak stress and most of the trials show class I behavior with decreasing axial stress in the discretized region. Strain-softening indicates that as cracks are produced, stress is transferred from the region covered with the lattice where cracking can occur to the uncracked region of the side borders.

1 These stress-strain curves reflect several assumptions incorporated in the field-theory model and the way the model was operated to simulate stress-stain behavior. In particular, no attempt is made to simulate or evaluate kinematic instability, and the loading rate is that of a soft loading machine. These and other relevant assumptions and methods are discussed in detail in Section 4.4. 
This figure shows that for these trials, peak strength consistently decreases as array size increases. This is also shown in Table 5.1-1. Stress-strain behavior for the $10 \times 30$ array shows nonlinear behavior at applied stresses of 16,22 , and $26 \mathrm{MPa}$ with the most strain-softening at $26 \mathrm{MPa}$, which also leads to failure. The stress-strain curve for the $20 \times 60$ array is similar to that for the $10 \times 30$ array, with strain-softening at an applied stress of $22 \mathrm{MPa}$, then a major strain-softening event at $24 \mathrm{MPa}$ which leads to failure. This trial also has the greatest amount of strain at failure of any of these trials. Stress-strain behavior for the $27 \times 81$ array follows the same deviation from linear as the smaller arrays, but a major episode of strainsoftening at $20 \mathrm{MPa}$ leads to failure. Finally, the stress-strain curve for the $30 \times 90$ array is similar to that for the $27 \times 81$ array, and follows the same deviation from linear behavior with increasing axial stress up to $16 \mathrm{MPa}$; then the first major episode of strain-softening leads to failure of the array. In summary, this figure shows that the larger arrays failed at lower levels of applied stress, and had more crack strain energy. Figure 5.1-1 also shows that the two smallest arrays show at least two significant intervals of strain-softening before the critical cluster is formed, while the first significant strain-softening interval for the two larger arrays leads to formation of the critical cluster and failure. It is interesting to note that for both the $10 \times 30$ and $20 \times 60$ arrays, significant strain-softening occurs at a stress of $26 \mathrm{MPa}$, but these do not cause failure at this stress level and additional stress is required to form the percolating cluster. This is discussed further in Chapter 6 and the strain-softening is shown to be caused by crack extension. Nonlinear behavior for all trials starts at about 6 to $12 \mathrm{MPa}$. The deviation from linear behavior with increasing axial stress is also consistent for all sizes. It is also interesting to note that the stress-strain behavior of the larger arrays is similar to the behavior of the homogeneous site strength arrays discussed in Section 5.1. This may imply that at sizes of approximately 2000 or more sites, heterogeneity in site strength may not affect stress-strain behavior. 


\section{Applied Stress vs Crack Strain Energy}

The partitioning of strain energy throughout the simulation can be used to gain insight into the process of fracture; applied stress vs normalized crack strain energy for these four trials is shown in Figure 5.1-2. This figure shows that normalized crack strain energy at failure increases as the array size increases. This means that as the array size increases a larger proportion of strain energy in the system at failure is due to cracks. All trials show a large increase in crack strain energy at the highest applied stress. The behavior shows fundamental changes as array size is changed. In particular, the behavior for the larger arrays shows that initially there is a small and nearly linear accumulation of crack strain energy with increasing applied stress, followed by a massive increase in crack strain energy at the applied stress that causes failure. In contrast, the smaller arrays show more crack strain energy at lower stress levels, and more significant gains in crack strain energy with applied stress as the stress is increased. Thus, the overall proportion of strain energy in cracks at failure increases with array size, and most of the accumulation occurs at one stress level. Increasing the array size causes less of the crack strain energy to be accumulated during the incremental loading and a higher proportion of the strain energy in the system at failure to be due to cracks. This may indicate that as the array size is increased, the onset of the growth of a critical cluster of cracked sites occurs more quickly and at lower levels of applied stress, and may mean that as array size is increased, crack interaction begins to dominate the behavior at lower stress levels. This may occur because in larger arrays cracked zones have more ways to interact, and a propagating crack will have more possibilities for extending and will find the weakest path through a material.

\section{Number of Cracks vs Crack Strain Energy}

Figure 5.1-3 shows the fraction of cracked sites vs the normalized crack strain energy for each of the four trials. Initially crack strain energy increases slowly and linearly as sites are cracked. This is consistent with the stress-strain behavior and indicates that the first few 
percent of cracks do not contribute to the overall macroscopic behavior. ${ }^{1}$ This stage represents cracking of randomly located sites. When approximately $10 \%$ of the sites are cracked the slope of the curves change dramatically, with much larger gains in crack strain energy occurring with each cracked site. Increasing gains in strain energy with increasing number of cracks indicates crack interaction and crack extension, while increasing the number of cracked sites without increasing normalized crack strain energy indicates crack arrest and cracking of randomly located sites. Data for all trials show that when normalized crack strain energy reaches values in the range 0.14 to 0.24 , individual cracked sites start contributing substantial amounts of crack strain energy and normalized crack strain energy increases dramatically. It is interesting to note that this transition occurs when approximately 13 to $16 \%$ of the sites are broken. This may indicate that crack interaction starts to dominate the behavior at this number of cracks. The relation between crack interaction and the strain energy plots is discussed in detail in Chapter 6.

\section{Crack Patterns}

Crack patterns at the percolation threshold (i.e., fracture) for the four trials are shown in Figure 5.1-4. This figure shows that while the crack patterns for the larger arrays are more realistic, all patterns have similar characteristics, with a population of dispersed cracked sites, a few small-to-medium clusters, and one large cluster containing en-echelon patterns of cracked sites. Moreover, in all of the trials macroscopic surfaces are formed that are similar to the cross sections of fractures formed by shear localization in real rocks under compression. The development of crack patterns for the $10 \times 30$ and $30 \times 90$ trials is discussed in more detail in Sections 5.3.2 and Chapter 6, respectively.

\footnotetext{
1 Note: this is for a heterogeneous site strength distribution.
} 


\subsubsection{Multiple Trials-Ultimate Stress and Percolation Threshold}

Summary data for the sets of trials used in this analysis are presented in Table 5.1-2.

Detailed data for the individual trials comprising the sets are presented in the Appendix.

Data for ultimate stress are plotted as a function of array size in Figure 5.1-5 and show that as indicated in Figures 5.1-1 and 5.1-2 the ultimate stress decreases with increasing array size. Increasing the array size also reduces its standard deviation, as expected. Both a power law and a linear fit have been used to describe the dependence of ultimate strength on array size, because even though the data appear to decline in an approximately linear manner, the linear fit indicates that at some array size the ultimate strength would decrease to zero, and this is not physically plausible in compression. It is important to note that the power-law exponent is low $(<0.2)$, and that the two larger sizes have similar ultimate strengths, and this may indicate that the mean ultimate strength is independent of size, especially at sizes greater than a few thousand sites.

Figure 5.1-5 shows that the field-theory model exhibits an implicit size effect and it is important to note that no assumption of flaw size is needed to generate this effect, and the effect is associated with a decrease with increasing size in the stress level required to create crack-interaction. The relation of this size effect to that observed in measurements on rock is discussed in Section 5.1.3.

Percolation thresholds were also examined as a function of size, and values for the four array sizes are given in Table 5.1-2 and are plotted in Figure 5.1-6. This figure shows that percolation thresholds decrease with increasing array size, with the largest size having a mean threshold of 0.18 . Moreover, the values are much lower than the theoretical value of 0.59 for random percolation on a triangular lattice. This indicates that the cracking is not a random process, but is localized and that crack interaction plays a major role that increases with increasing size. Again, power law and linear fits were used to describe the dependence of percolation threshold on array size. The power-law relation is more reasonable because a lower bound of the threshold does exist. Moreover, the exponent is small, indicating a weak 
dependence on size as size increases. The low percolation thresholds are consistent with the analysis of Bebbington et al. (1990), who conclude that variations on percolation theory may be appropriate for analysis of rock fracture.

Table 5.1-2. Ultimate strength and percolation thresholds for simulations with different lattice sizes. All trials were at baseline conditions with $p_{c}=0.1, m_{1}$ in \pm 0.7 .

\begin{tabular}{ccccc}
\hline $\begin{array}{c}\text { Array } \\
\text { dimensions }\end{array}$ & $\begin{array}{c}\text { Number of } \\
\text { sites }\end{array}$ & $\begin{array}{c}\text { Number of } \\
\text { trials }\end{array}$ & $\begin{array}{c}\text { Ultimate stress } \\
(\mathrm{MPa})\end{array}$ & $\begin{array}{c}\text { Percolation } \\
\text { threshold }\end{array}$ \\
\hline $10 \times 30$ & 300 & 10 & $34.1 \pm 3.3$ & $0.33 \pm 0.04$ \\
$20 \times 60$ & 1200 & 10 & $31.1 \pm 1.3$ & $0.25 \pm 0.05$ \\
$27 \times 81$ & 2175 & 7 & $24.6 \pm 1.6^{\mathrm{a}}$ & $0.22 \pm 0.02^{\mathrm{b}}$ \\
$30 \times 90$ & 2700 & 8 & $22.6 \pm 3.1^{\mathrm{a}}$ & $0.18 \pm 0.003^{\mathrm{c}}$ \\
\hline
\end{tabular}

${ }^{a}$ All trials did not form critical cluster. ${ }^{b}$ Based on 5 values. ${ }^{c}$ Based on 3 values.

Table 5.1-2 also shows that for the $30 \times 90$ and $27 \times 81$ sizes computation of mean values was based on results of three and seven trials, respectively. Ten trials were attempted for each of these sizes, but due to the limitations on the computational facilities all trials did not run to completion. This is because of the limit on the available RAM memory of the computers used (256 MB). Thus, the trials that did complete are the ones that have ultimate strengths and percolation thresholds that are lower than would be predicted if all trials had completed. However, these results show trends that are useful in describing the effect of lattice size.

If the model is assumed to represent a sandstone with grains that have average diameter of 100 microns, then the $30 \times 90$ size represents a piece of the sandstone that is $0.3 \mathrm{~cm}$ by $0.9 \mathrm{~cm}$ in size. Specimens of sandstone used in common laboratory compression tests have many tens of thousands of grains.

\subsubsection{Discussion of Lattice Size Effect}

The relationship of sample size to observed strength for rock is important in rock engineering, because while most rock strength data are from laboratory measurements on intact 
core samples, the primary use of these data is to assess the behavior of large volumes of rock in field applications such as estimating mine pillar strength and excavation support requirements. Several authors have reviewed work on the effect of size on compressive strength (Bieniawski and Van Heerden, 1975; Jaeger and Cook, 1976; Hoek and Brown, 1980; and Goodman, 1980) and find that the strength of rock samples tested in compression generally decreases with the size of the sample tested, following an inverse power law. The power-law relation

$$
\sigma_{p k}=c D^{-\vartheta}
$$

relating peak strength to sample diameter (or edge length), $D$, where $c=$ a constant, was first proposed by Evans and Pomeroy (1958) and Evans et al. (1961). Other studies have shown that the values of the exponent $\vartheta$ range from 0.17 for coal (Evans and Pomeroy, 1958) to 0.8 for welded tuff (Price, 1986).

Hoek and Brown (1980) reviewed several studies of the size effect on sample strength and formulated an alternative power law

$$
\sigma_{\mathrm{pk}}=\sigma_{\mathrm{pk}_{50}}\left(\frac{50}{D}\right)^{0.18}
$$

where $\sigma_{\mathrm{pk}_{50}}$ is the strength of samples with diameter of $50 \mathrm{~mm}$. Figure 5.1-5 shows that the field-theory model predicts an inverse power-law dependence of strength on size with an exponent of approximately 0.18 , which is in good agreement with that observed on real rock in the studies discussed above. Moreover, the size effect is implicit in the model as no assumption is made regarding flaw size.

The decrease in strength with increasing size may be due to the increase in number and type of interactions between cracks and crack clusters that is provided by the larger population of cracked sites in the bigger arrays, so that bigger arrays offer more ways to form a weak path through the array. It is interesting to note that the percolation threshold also decreases with increasing size. These effects are a consequence of crack interaction and indicate that in larger arrays crack interaction starts to dominate the behavior at lower levels of applied stress, and at a lower fraction of sites broken. 


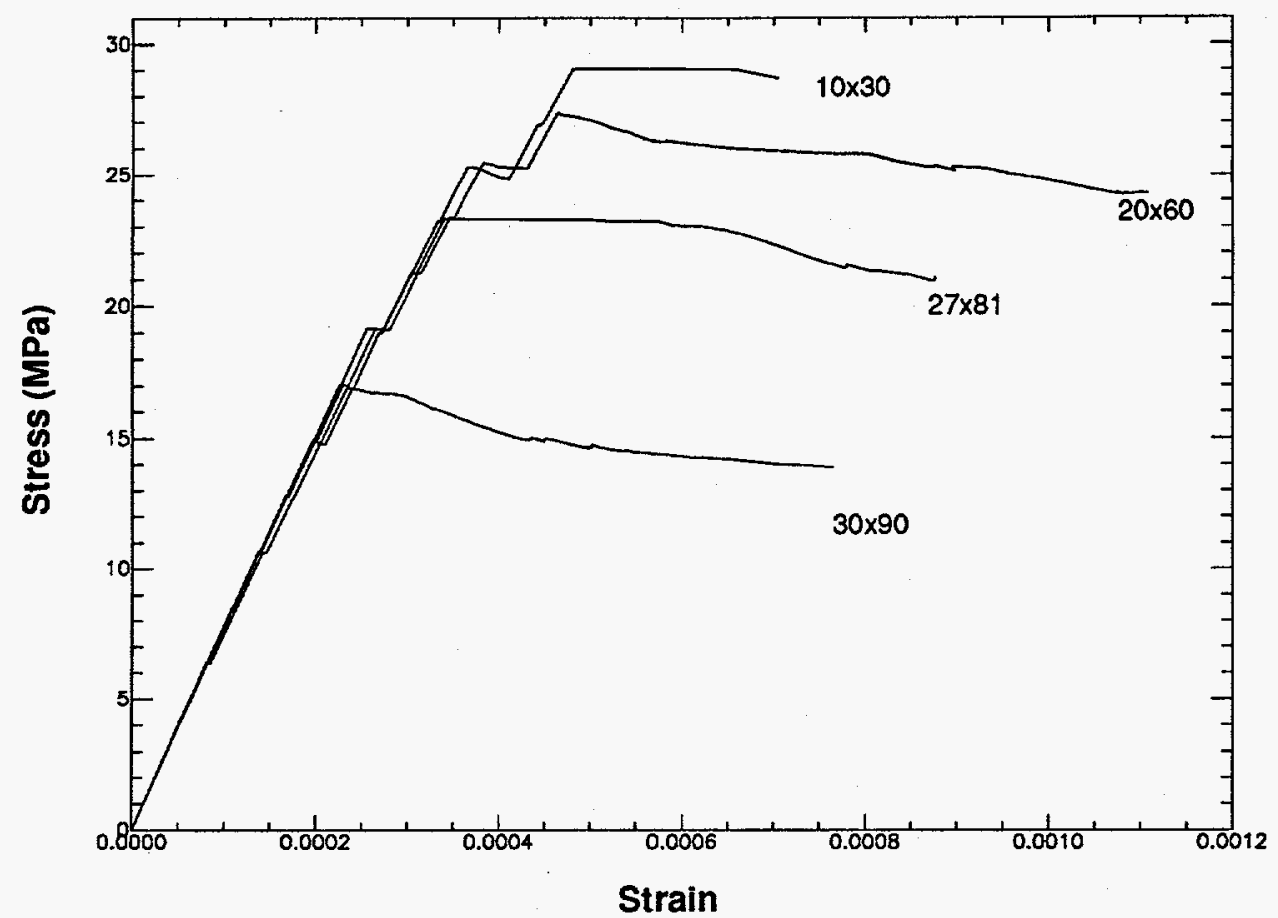

Figure 5.1 - 1 Effect of lattice size on stress-strain behavior for triangular lattices

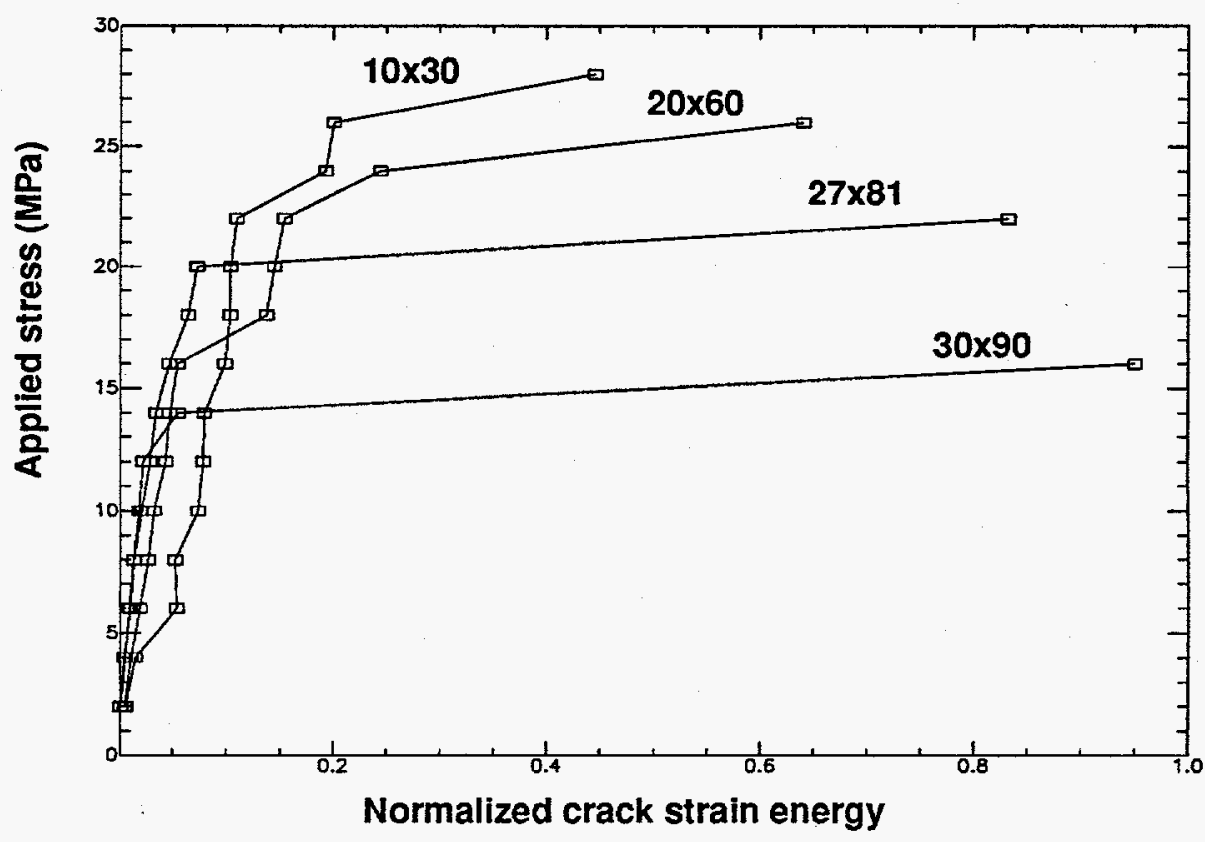

Figure 5.1 - 2 Effect of lattice size on relation of applied stress to normalized crack strain energy for triangular lattices 


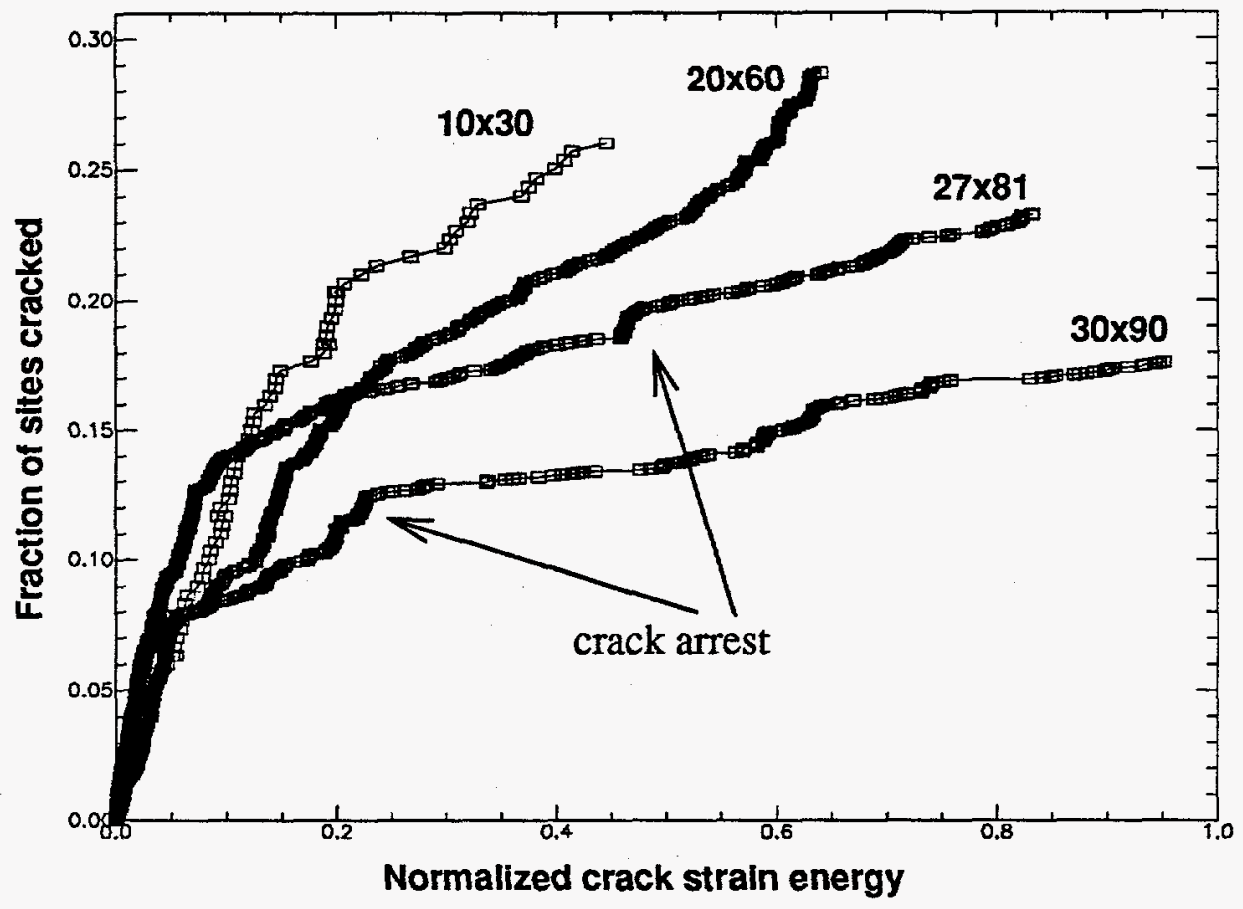

Figure 5.1 - 3 Effect of lattice size on relation of fraction of cracked sites to crack strain energy for triangular lattices. 


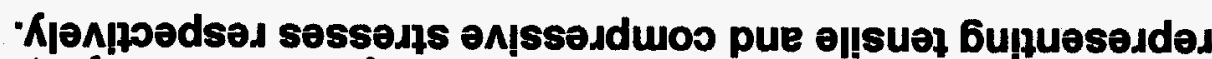

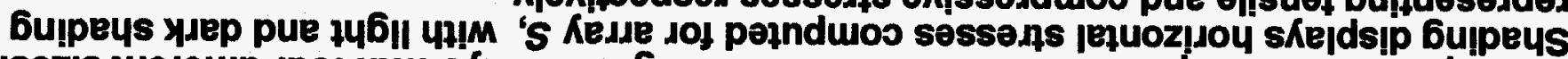

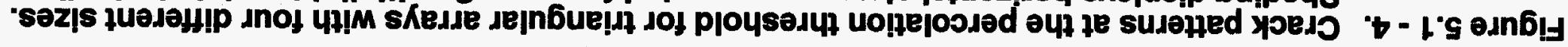

$06 \times 0 \varepsilon(p$

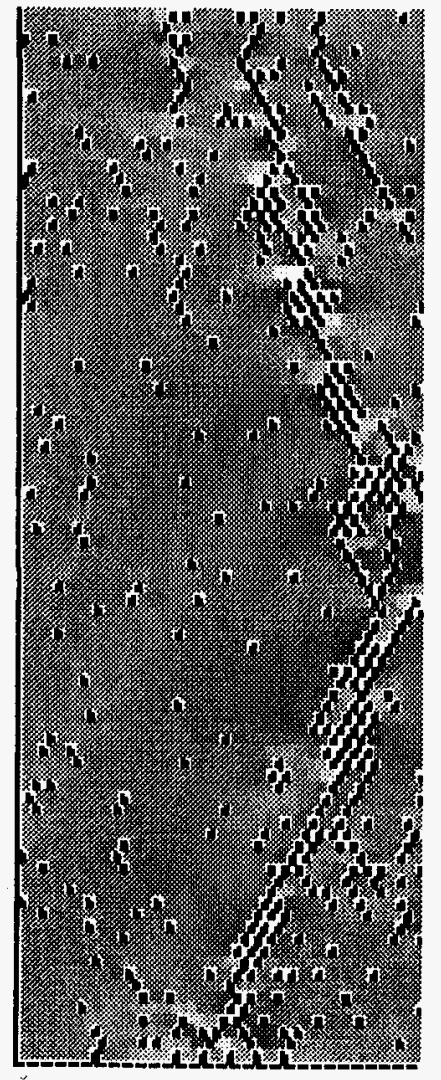

9l-
$18 \times 22(0$

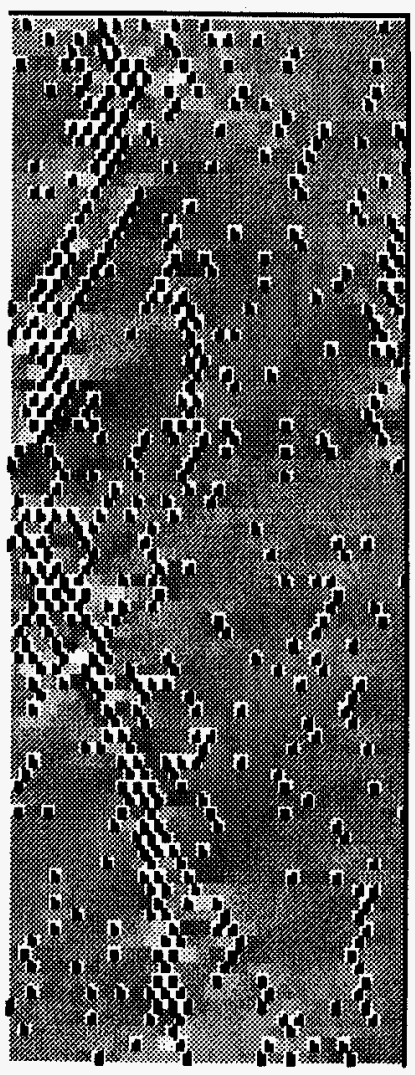

ZZ-
$09 \times 02$ (q

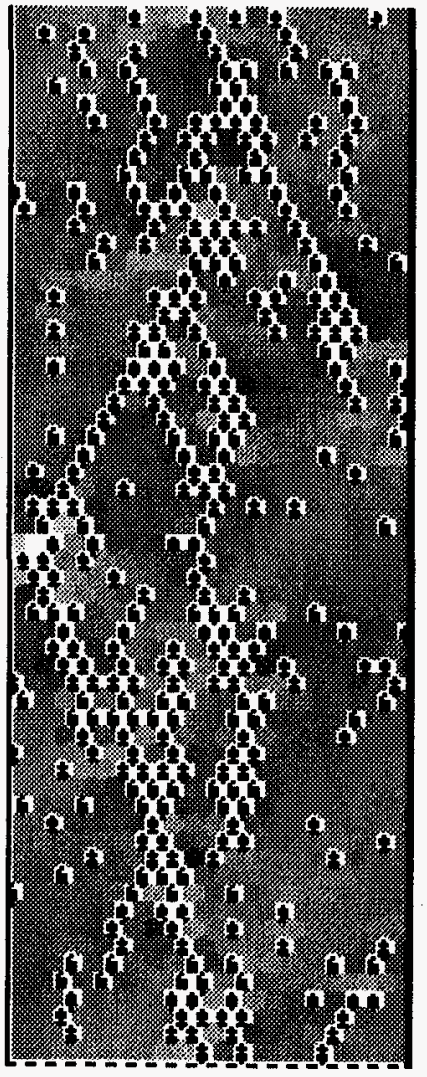

92-
OExol (e

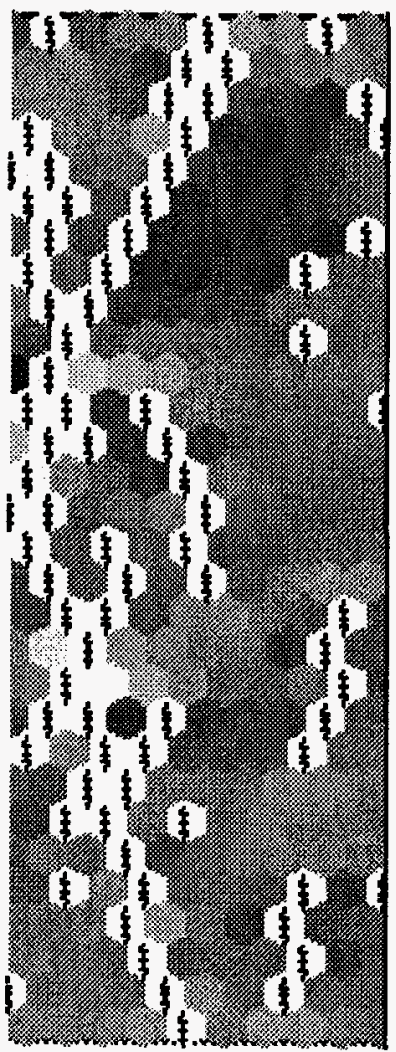




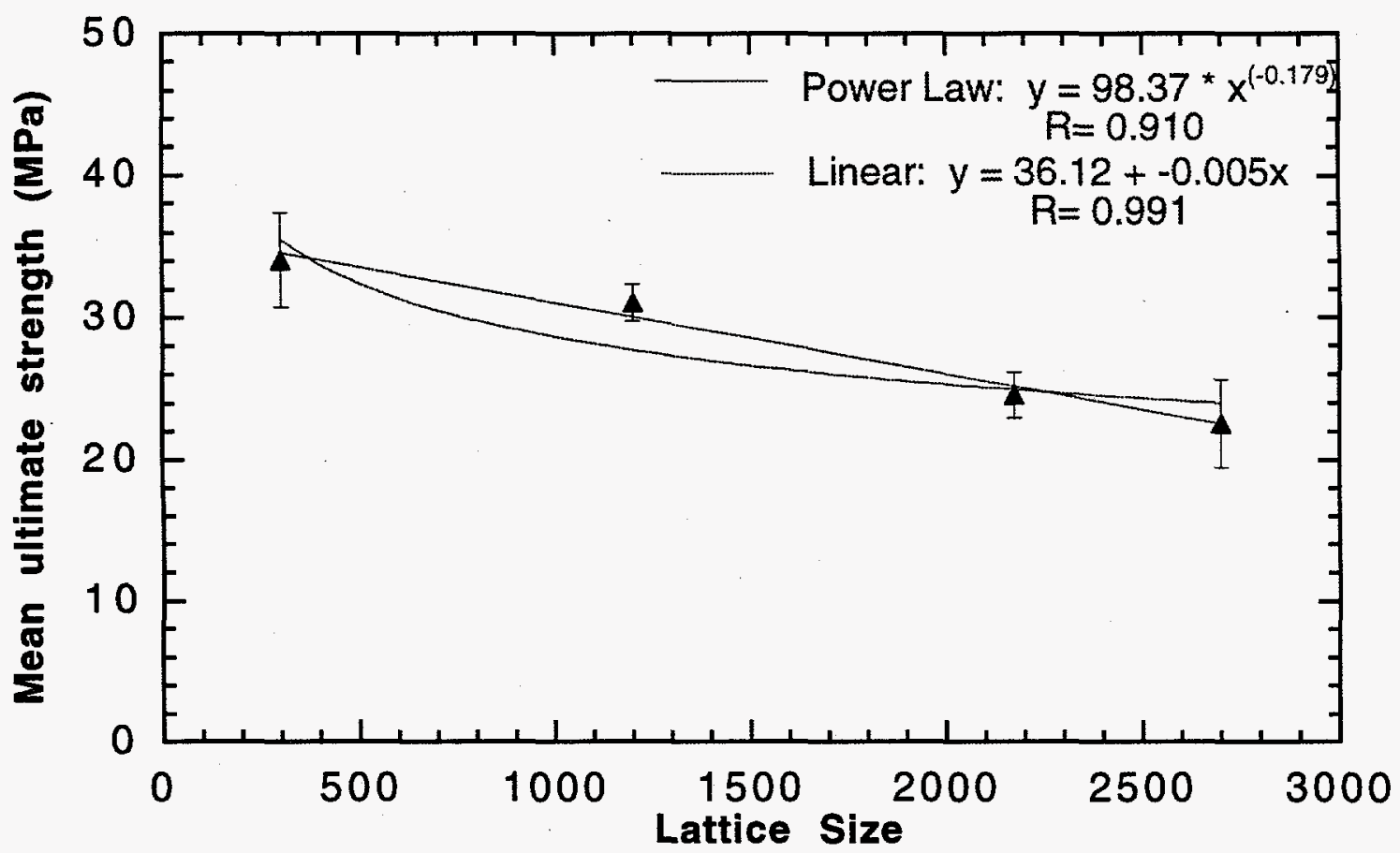

Figure 5.1-5. Dependence of ultimate strength on lattice size for triangular lattices.

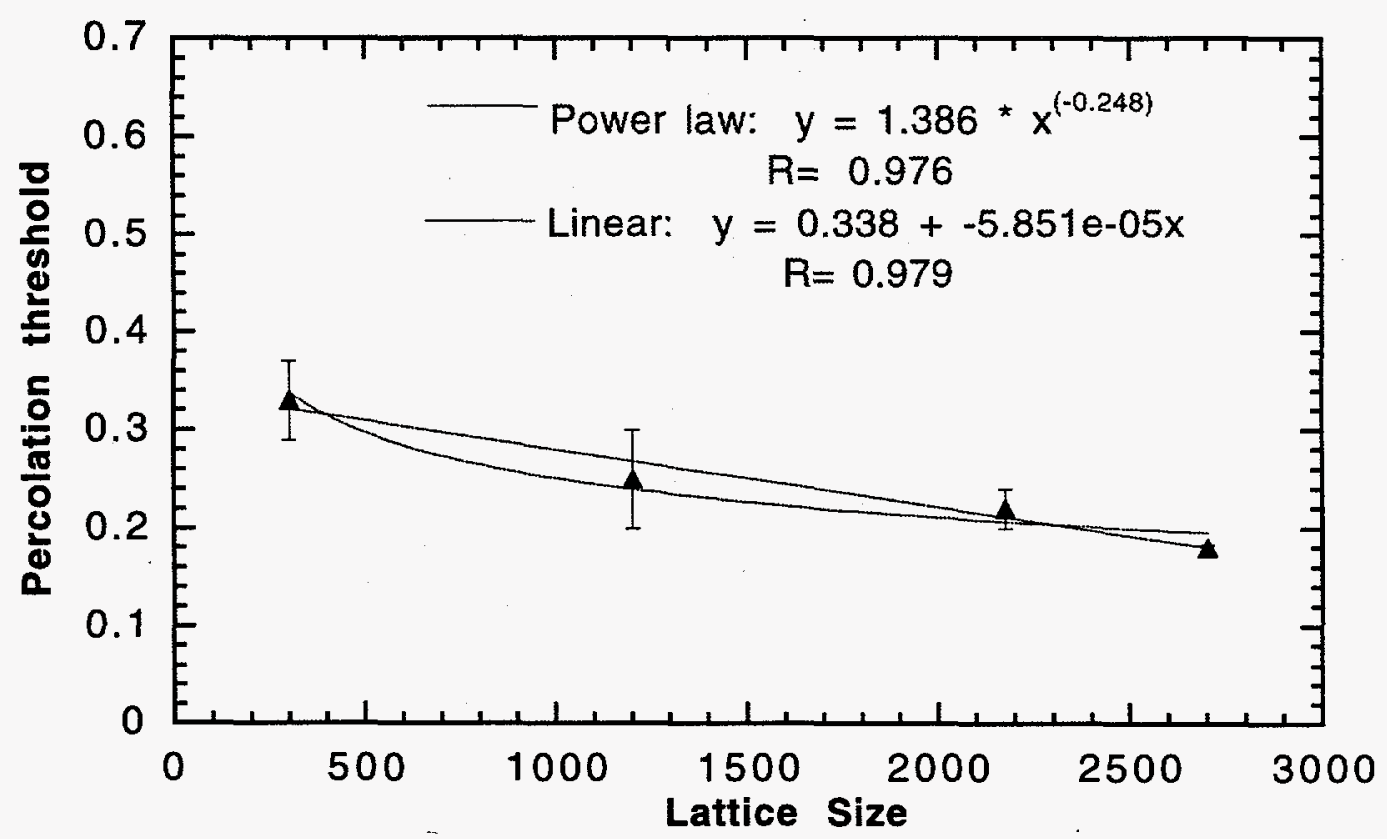

Figure 5.1-6. Dependence of percolation threshold on lattice size for triangular lattices. 


\subsection{Effect of Microscale Stress Heterogeneity: Triangular Lattice}

The purpose of this section is to investigate how the microscale stress heterogeneity affects the macroscopic behavior. The stress perturbation parameter, $\rho$, is designed to incorporate variation in the shape and loading of grains into the field-theory model. Heterogeneity in this parameter is determined by the distribution of the stress difference multiplier $m_{1}$, and changing the range of $m_{1}$ simulates changing the heterogeneity in the microgeometry of grains. This is because increasing the range of $m_{1}$ increases the range of stress perturbation at a given level of applied stress, making more of the stress difference available for production of cracks. This is similar to introducing grains with a wider variety of shapes and loading geometries. To investigate the effect of local stress heterogeneity, sets of 10 uniaxial trials were conducted with $m_{1}$ in the ranges $\pm 0.3, \pm 0.5, \pm 0.7$ for two different site strength distributions (called homogeneous and heterogeneous site strengths). ${ }^{1}$ Ten trials were also computed with $m_{1}$ in the range \pm 1.0 using a heterogeneous strength distribution. Results of these trials are analyzed in two ways. First, the general effect of local stress heterogeneity is illustrated by discussing the behavior of four single trials, each computed using a different range of $m_{1}$ values, but with equivalent heterogeneous site strength distributions of $T_{g}$ in $20 \pm 19.9 \mathrm{MPa}$. Then, data from the sets of trials are presented. These data describe the dependence of average properties such as mean ultimate strength and mean percolation threshold on the range of $m_{1}$.

\subsubsection{Single Trials}

Results for four single trials are presented in this section, one from each of the sets conducted using heterogeneous site strength distribution and $m_{1}$ in the ranges $\pm 0.3, \pm 0.5, \pm 0.7$, and \pm 1.0 . A heterogeneous site strength distribution was used for these trials with a mean site strength of $20 \mathrm{MPa}$ and a distribution width of $\pm 19.9 \mathrm{MPa}$. The structure of this subsection is as follows: first, stress-strain curves are presented, followed by applied stress vs normalized

\footnotetext{
${ }^{1}$ Detailed discussion of site strength distributions is presented in Section 5.3.
} 
crack strain energy, number of cracked sites vs normalized crack strain energy, and finally patterns of cracked sites. Summary data for these trials are presented in Table 5.2-1.

Table 5.2-1. Data for single trials for different ranges of $m_{1}$.

\begin{tabular}{|c|c|c|c|c|c|c|c|c|c|c|c|}
\hline Date & Lat. $^{\mathbf{a}}$ & JSZ & ISZ & $\begin{array}{c}\text { Init. } \\
\sigma_{\mathrm{I}}\end{array}$ & $\Delta \sigma_{\mathrm{I}}$ & $T_{g}$ & $m_{1}$ & $\mathbf{v}^{\mathbf{b}}$ & seq & $\begin{array}{c}\sigma_{\mathrm{pk}} \\
(\mathbf{M P a})\end{array}$ & $p_{c}$ \\
\hline 15-Jun-94 & tri & 10 & 30 & 10 & 2 & $20 \pm 19.9$ & .3 & 35.1 & 2 & 73.0 & 0.28 \\
\hline 20-May-94 & tri & 10 & 30 & 10 & 2 & $20 \pm 19.9$ & .5 & 35.7 & 8 & 42.9 & 0.29 \\
\hline 29-Jun-94 & tri & 10 & 30 & 10 & 2 & $20 \pm 19.9$ & .7 & 35.7 & 1 & 31.9 & 0.31 \\
\hline 15-Jun-94 & tri & 10 & 30 & 10 & 2 & $20 \pm 19.9$ & 1.0 & 35.10 & 4 & 17.0 & 0.29 \\
\hline
\end{tabular}

a Lattice type. $b$ Version of program.

\section{Stress-Strain}

The effect of increasing the range of $m_{1}$ on stress-strain behavior is illustrated in Figure 5.2-1. This figure shows that altering the range of $m_{1}$ has a large and significant effect on the stress-strain behavior. In particular, while strain at failure is similar for three of the four trials, there is a large variation in peak strength. The trial with the smallest range in $m_{1}$ $\left(m_{1}\right.$ in \pm 0.3$)$ has the highest peak strength $(73 \mathrm{MPa})$, and the trial with the largest range of $m_{1}$ ( $m_{1}$ in \pm 1.0 ) has the lowest peak strength (17 MPa). Also, peak strength appears to decrease monotonically with increasing range of $m_{1}$. Thus, relatively small changes in the range of $m_{1}$ have a large effect on the macroscopic behavior. Moreover, the nature of the stress-strain curves change as the range of $m_{1}$ is changed. All of the curves show sequences of strainsoftening/strain-hardening/strain-softening, but the amount of deviation from linear elastic increases with increasing range of $m_{1}$, as does the stress at which the curve deviates from linear elastic. The curve for $m_{1}$ in \pm 0.3 shows linear elastic deformation up to an applied axial stress of nearly $50 \mathrm{MPa}$, and substantial nonlinearity does not begin until applied stress is greater than $60 \mathrm{MPa}$. The stress-strain curve for the trial with $m_{1}$ in \pm 0.5 shows a peak stress of 
approximately $43 \mathrm{MPa}$ and shows significant nonlinear behavior at stresses above about $25 \mathrm{MPa}$. The stress-strain curve for the trial with $m_{1}$ in \pm 0.7 shows nonlinear behavior starting at about $10 \mathrm{MPa}$ and substantial strain-softening at an applied stress of approximately $28 \mathrm{MPa}$. This was followed by some strain-hardening before the critical cluster formed, causing failure. The total strain computed for this trial was similar to that computed for the trial with $m_{1}$ in \pm 0.3 , but the level of applied stress is less than half that for the \pm 0.30 trial. The stress-strain curve for the trial with $m_{1}$ in \pm 01.0 shows behavior similar to that for the \pm 0.7 trial, but the ultimate stress is lower and substantial strain-softening occurs at an applied stress of $10 \mathrm{MPa}$.

\section{Applied Stress vs Normalized Crack Strain Energy}

Figure 5.2-2 shows the applied stress plotted vs the normalized crack strain energy for these four trials. This plot clearly illustrates that increasing the range of $m_{1}$ reduces the peak stress and increases the percent of strain energy contained in cracks. This lends insight into difference in the stress-strain curves shown in Figure 5.2-1, which shows very different behavior for trials with $m_{1} \pm 0.3$ and $m_{1} \pm 1.0$, but similar amounts of strain at failure. For the $m_{1} \pm 0.3$ case, most of the strain is from the linear elastic portion of the curve and a smaller component is from the nonlinear behavior, while for the $m_{1} \pm 1.0$ case, very little of the total strain is from the linear portion of the curve and most of the strain is due to deviation from linear behavior. Thus for the $m_{1} \pm 0.3$ case, the elastic strain energy is large and dominates the strain energy due to cracked sites, and the normalized value of crack strain energy is low. For the $m_{1} \pm 1.0$ case the opposite is true, as the elastic strain energy is small and most of the strain energy is due to site cracking, so the normalized crack strain energy is quite high.

Thus this indicates that the geometry of grains may have a large effect on the way in which energy is partitioned in the fracturing process. That is, increasing the range of $m_{1}$ increases the magnitude of stress perturbations, and increases the amount of crack strain energy relative to the elastic strain energy at a given stress level. Put another way, this shows that for the wider ranges of $m_{1}$ (more heterogeneity in local stress) a higher proportion of strain 
energy is contained in cracks. The implication of this is that heterogeneity in the local stress field due to variations in the shape and loading of grains may provide a mechanism for more of the applied stress to be used to break up a rock. This is because, with larger values of $m_{1}$, cracking occurs at lower stress levels (see equation 4.15).

\section{Number of Cracked Sites vs Normalized Crack Strain Energy}

The number of cracks vs normalized crack strain energy is plotted in Figure 5.2-3. This figure shows a similar number of cracked sites for each of the four trials, and this is reflected in the percolation thresholds listed in Table 5.2-1. The number of cracked sites at the percolation threshold computed for the \pm 0.3 and \pm 0.5 trials were similar, as were the values for the trials with $m_{1}$ in \pm 0.7 and \pm 1.0 . In addition, for the \pm 0.7 and \pm 1.0 trials, crack interaction became significant when approximately 35 sites $(10 \%)$ were broken, as is evidenced by the change in slope of these curves at that number of cracks, while crack interaction did not become significant for the \pm 0.3 and \pm 0.5 cases until over 70 sites (20\%) were broken. (It is important to note that

other results for $m_{1}$ in \pm 0.5 show crack interaction at lower numbers of cracks, e.g., Figure 5.3-12.) This figure also illustrates that for trials with the same proportion of cracked sites, trials with a wider range of $m_{1}$ had more normalized crack strain energy. This may imply that increasing the local stress heterogeneity enhances crack interaction.

\section{Crack Patterns}

Crack patterns at failure for the same four trials are shown in Figure 5.2-4. These patterns are similar for all four trials and show no clear dependence on the range of $m_{1}$. However, more pronounced en-echelon cracking is shown for the trials with wider $m_{1}$ distributions, but more study is necessary to confirm this observation. The progressive development of crack patterns for trials 4 and 10 is presented in Figures 5.3-12 $a$ and $b$ in Section 5.3.2.

\subsubsection{Multiple Trials}

In addition to the single trials discussed above, sets of 10 trials were computed with values of $m_{1}$ in several ranges for both homogeneous and heterogeneous site strength 


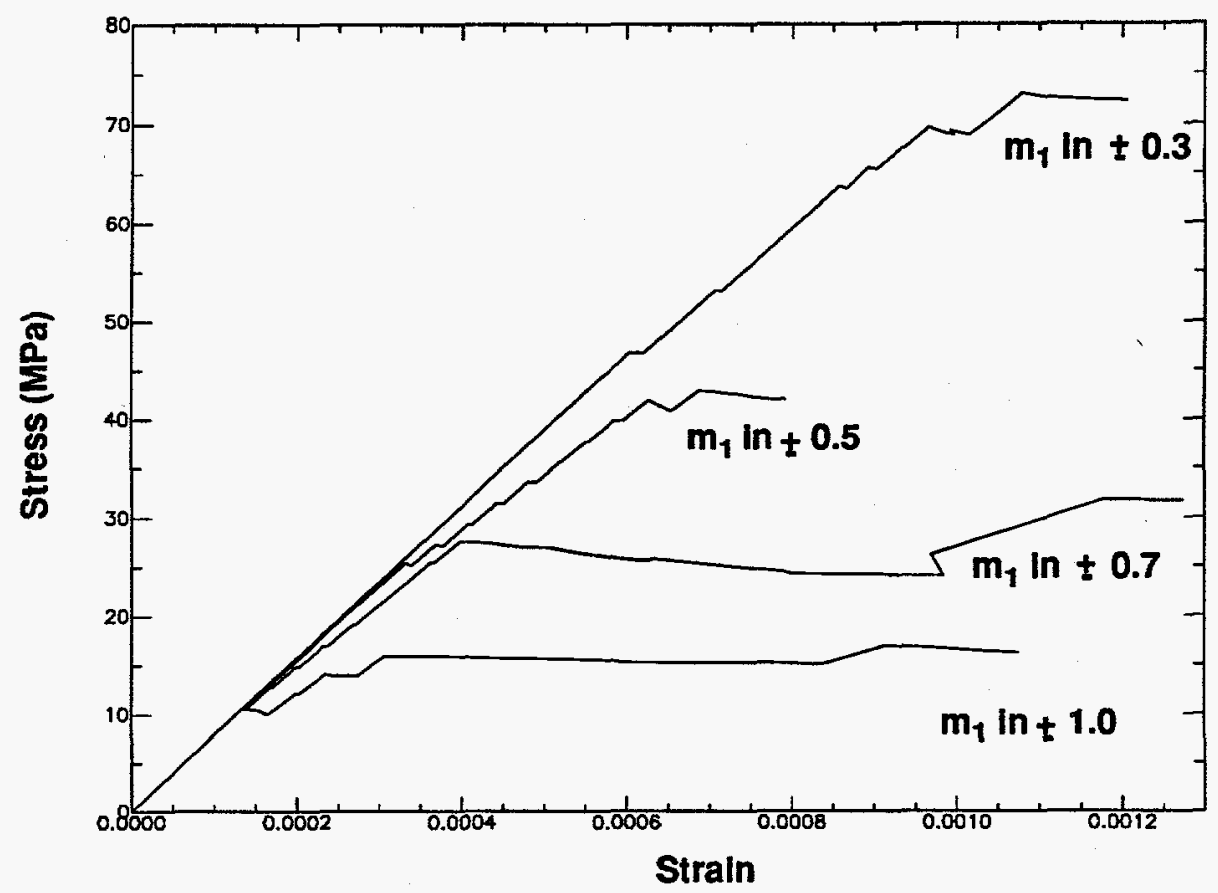

Figure 5.2 - 1 Effect of changing the range of the stress difference multiplier on stress-strain behavior

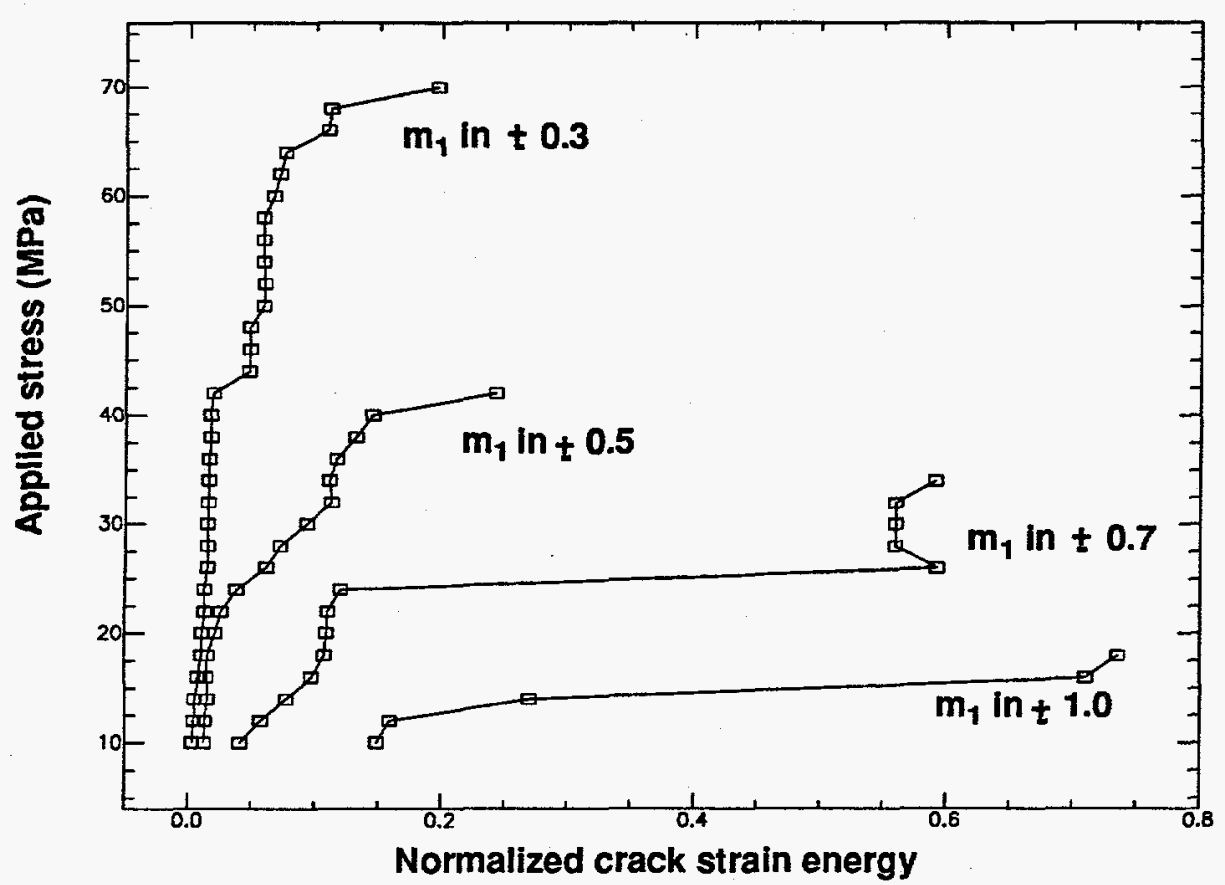

Figure 5.2-2 Effect of changing the range of the stress difference multiplier on the relation of applied stress to normalized crack strain energy. 


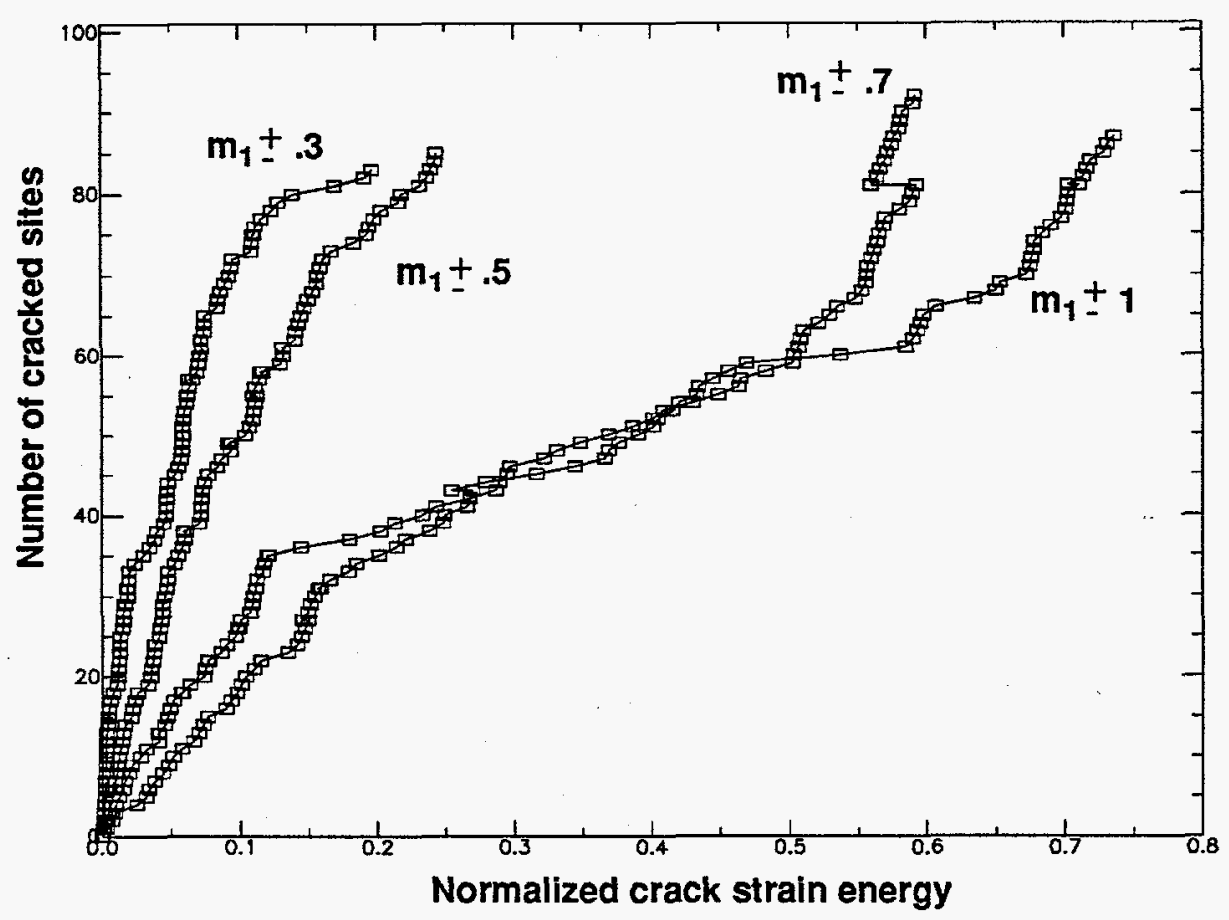

Figure 5.2 - 3 Effect of changing the range of the stress difference multiplier on the relation of number of cracked sites to normalized crack strain energy. 


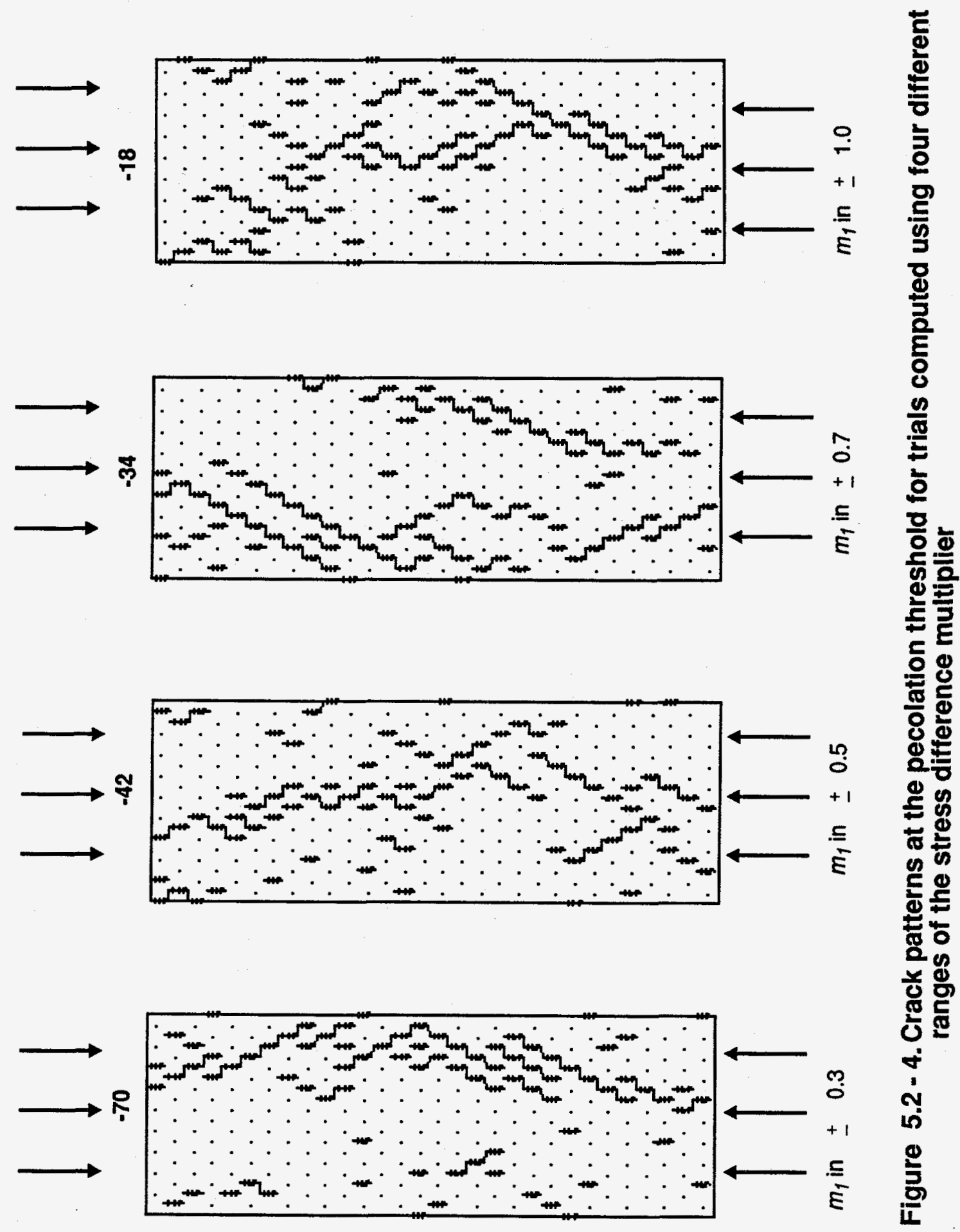


distributions. ${ }^{1}$ In this section, results of these sets of trials are discussed to show the dependence of the mean values of ultimate strength and percolation threshold on the range of $m_{1}$. Data for mean ultimate strength and percolation threshold for the sets of trials are tabulated in Table 5.2-2. Data for individual trials can be found in the Appendix.

Results for trials with a mean strength of $20 \mathrm{MPa}$ are plotted in Figure 5.2-5. This figure shows that for uniaxial simulations: (1) the average ultimate strength has a strong inverse dependence on the range of $m_{1}$, with ultimate strength decreasing as the range in $m_{1}$ increases. The width of the error bars also decreases with increasing $m_{1}$; (2) site strength distribution has a relatively small effect on the peak strength over this range of $m_{1}$ values; (3) the dominant effect of $m_{1}$ can be attributed in part to the fact that it is a direct multiplier of the stress difference, and reducing the range of $m_{1}$ (about a mean of zero) reduces the range of values that the stress perturbation term can acquire at any given stress condition (see equation 4.8).

Table 5.2-2. Summary data for $m_{1}$ trials.

\begin{tabular}{lccccc}
\hline $\begin{array}{c}\text { Site strength } \\
\text { distribution }\end{array}$ & $\begin{array}{c}\text { Mean site } \\
\text { strength (MPa) }\end{array}$ & $m_{1}$ & $\begin{array}{c}\Delta \sigma_{1} \\
(\mathrm{MPa})\end{array}$ & $\begin{array}{c}\sigma_{\mathrm{pk}} \\
(\mathrm{MPa})\end{array}$ & $p_{c}$ \\
\hline $20 \pm 0.2$ & 20 & .3 & 10 & $74.8 \pm 0.0$ & $0.22 \pm 0.03$ \\
$20 \pm 19.9$ & 20 & .3 & 2 & $68.9 \pm 14.2$ & $0.30 \pm 0.06$ \\
$20 \pm 0.2$ & 20 & .5 & 2 & $42.4 \pm 2.5$ & $0.18 \pm 0.03^{\mathrm{a}}$ \\
$20 \pm 19.9$ & 20 & .5 & 2 & $41.4 \pm 2.8$ & $0.29 \pm 0.03^{b}$ \\
$20 \pm 0.2$ & 20 & .7 & 10 & $31.4 \pm 0.0$ & $0.22 \pm 0.05$ \\
$20 \pm 19.9$ & 20 & .7 & 10 & $34.1 \pm 3.3$ & $0.31 \pm 0.03$ \\
$20 \pm 19.9$ & 20 & 1.0 & 1 & $22.2 \pm 7.3$ & $0.33 \pm 0.07$ \\
\hline
\end{tabular}

a 8 values used. $b 9$ values used.

1 Site strength distributions are discussed in detail in Section 5.3. 


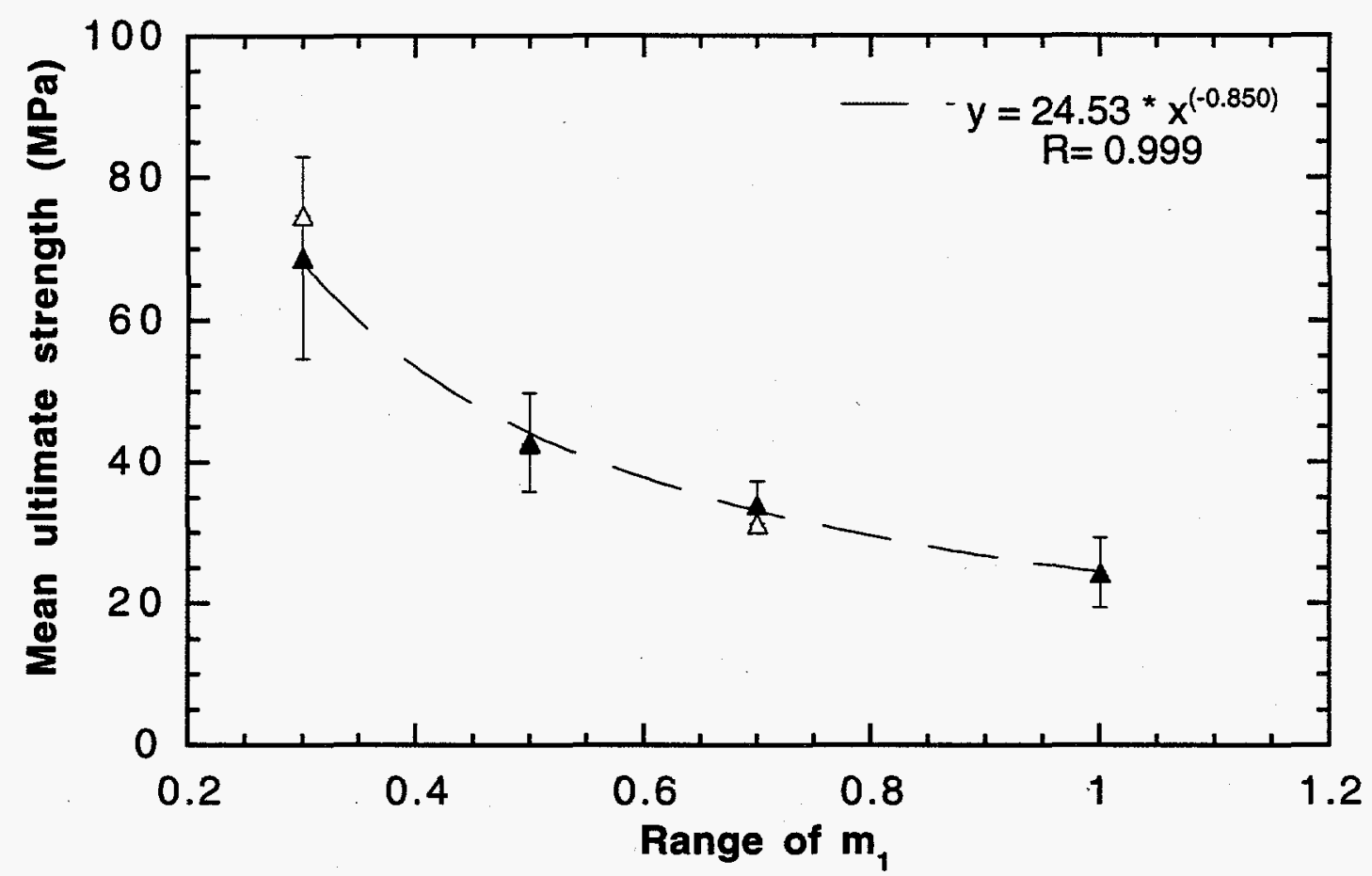

$\Delta \quad$ Homogeneous site strength $\longrightarrow$ - Heterogeneous site strength

Figure 5.2-5. Effect of changing the range of the stress difference multiplier, $m_{1}$, on mean ultimate strength. 
Accordingly, for narrower ranges of $m_{1}$, higher stress differences are required to overcome a given tensile strength. Moreover, for homogeneous site strength distributions, when all sites have similar tensile strengths, decreasing the range of $m_{1}$ means that higher stresses are required to initiate cracking. As the range in $m_{1}$ is increased, lower values of stress difference are required to produce the needed stress perturbation to fail a site. However, results for the heterogeneous site strength distributions yield ultimate strengths very similar to the homogeneous site strength trials for each of the three ranges of $m_{1}$ where both site strength distributions were computed. This is consistent with the results reported in Section 5.3, and is indication that for a given range of $m_{1}$ values, the ultimate stress may be determined by the mean site strength, at least over this range of $m_{1}$ values. This result is consistent for all of the site strength and geometric distributions used, even though for heterogeneous site strength distributions more cracks are formed at low values of applied stress.

The ultimate strength displays an inverse power-law dependence on the range of $m_{1}$, and this implies an inverse power-law dependence on the amount of heterogeneity in local stress. Moreover, the inverse dependence means that larger amounts of heterogeneity have less effect. This is consistent with the observation that small amounts of geometric heterogeneity are important in pure materials. Also, this plot shows that the ultimate strength is quite sensitive to $m_{1}$ in the range of distributions that are expected in rocks (see discussion in Section 4.1.3).

This has implications for anisotropy in strength, because when grains are preferentially oriented in one direction the local stress concentrations may become spatially correlated, and the rock may be much weaker or stronger in a particular direction.

\subsubsection{Summary of Stress Perturbation Effect}

Results reported in this section indicate that heterogeneity in local stress perturbation due to grain shape and loading has a large effect on macroscopic properties. In particular:

(1) A first-order result is that increasing heterogeneity in local stress lowers mean ultimate strength; this follows an inverse power law. 
(2) Increasing the heterogeneity in local stress makes the stress-strain curves more realistic. This may be because changing the amount of heterogeneity in local stress also changes how energy is partitioned; increasing local stress heterogeneity causes crack interaction to become dominant at much lower levels of applied stress, and a larger proportion of energy to go into crack formation.

(3) Increasing local stress heterogeneity had no significant effect on percolation threshold. Implications for real rocks and other materials are that for rocks with different grain shapes and loading distributions and the same matrix modulus, such as clean well sorted sandstones, the resulting heterogeneity in local stress actually homogenizes the behavior, as cracks are stopped and then must restart. More sites are available for restarting and it is easier to start another crack. Moreover, more cracks are allowed to form at lower stresses, which reduces the modulus. More energy goes into crack formation, and crack interaction occurs at lower stresses. Thus increased heterogeneity in local stress reduces both the mean peak strength and the effective modulus.

This result may provide an approach for improved use of petrographic analysis of rock in assessing rock behavior, and also has implications for design of materials in that this analysis indicates that the angularity and aspect ratio of particles in a material have a first-order effect on material strength.

The preliminary results reported here indicate that the local stress heterogeneity associated with grain shape and/or loading is much more important than the microscale heterogeneity associated with strength of sites, or site location. This has implications for researchers in the statistical modeling community who often use site strength as a parameter for introducing local scale heterogeneity into an analysis, as this result indicates that site strength may not be the appropriate parameter to use. However, only one distribution of the stress difference multiplier, $m_{1}$, was used (i.e., uniform distribution over narrow and wide ranges). Other distributions of $m_{1}$ should be evaluated, including multimodal distributions where the peak values are chosen based on observed shapes and loading geometries of grain in 
rock. In addition, only one formulation for stress perturbation at a site was used. Other formulations have been proposed, including the Kemeny and Cook (1987) expression used in Chapter 3 , in which the stress difference term is given by $m_{1}\left(\sigma_{1}-c_{2} \sigma_{2}\right)$. Using this expression, hydrostatic loading can cause a stress difference and local cracking. In the field-theory model (Chapter 4$), c_{2}=1$ was used.

\subsection{Effect of Heterogeneity in Site Strength: Triangular Lattice}

This section presents results that show how heterogeneity in site strength affects macroscopic behavior. Three different types of site strength distributions are examined, including: uniform distribution with narrow width about a mean, called the homogeneous site strength distribution; uniform distribution with wide width about a mean, called heterogeneous site strength distribution; and bimodal distribution. In addition, these distributions were used with different mean strengths. Typical examples of several of the site strength distributions listed in Table 5.0-1 are presented in Figure 5.3-1. In all cases the strengths for lattice sites were picked randomly from the appropriate distribution. Uniform and bimodal distributions were chosen after preliminary trials using these and normal (gaussian) distributions showed that these distributions were bounding cases, and that trials run using normal distributions did not show any behaviors not found using uniform distributions.

To show how heterogeneity in site strength distribution affects the macroscopic behavior in the simulated compression tests, it is useful to first examine the behavior when there is very little site strength heterogeneity and then introduce heterogeneity of different types and amounts. Thus, discussion in this section begins with results for trials with homogeneous site strength distribution, then progresses to heterogeneous site strength distribution, follows with bimodal site strength distribution, and finally gives results showing how variation in mean site strength affects behavior. 
a)

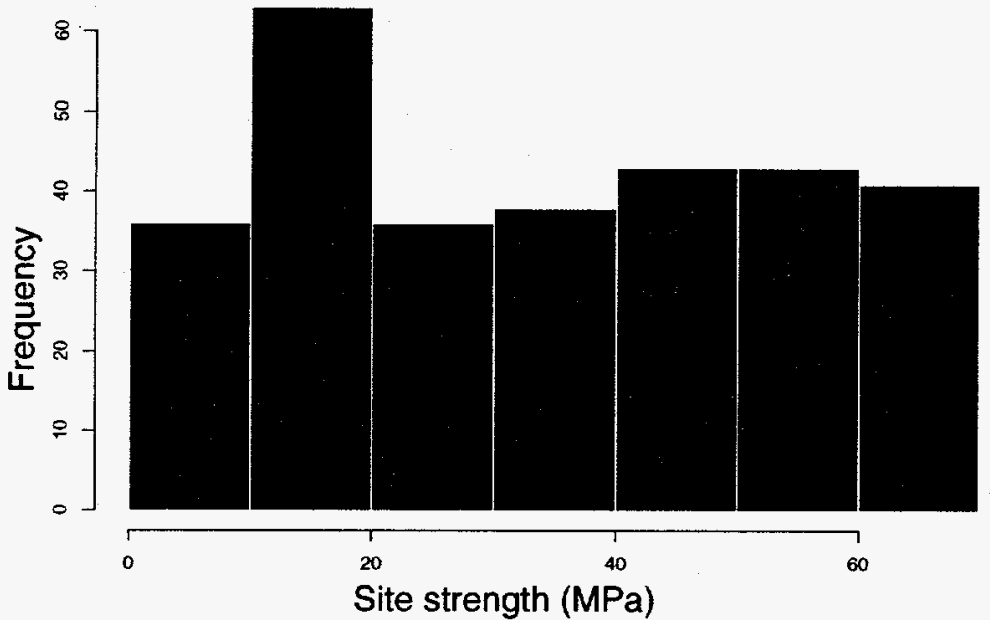

b)

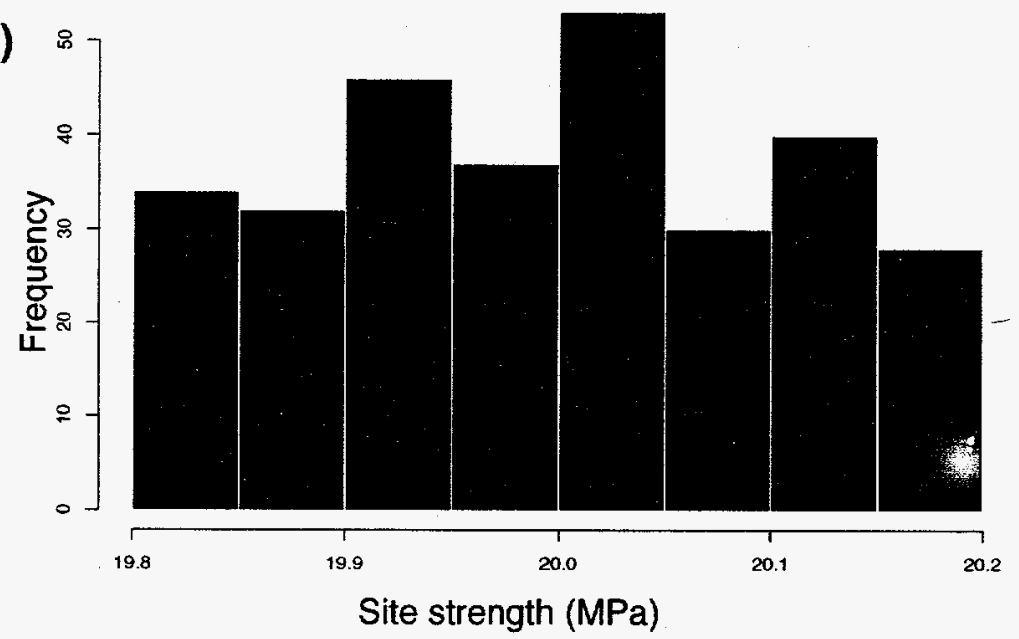

c)

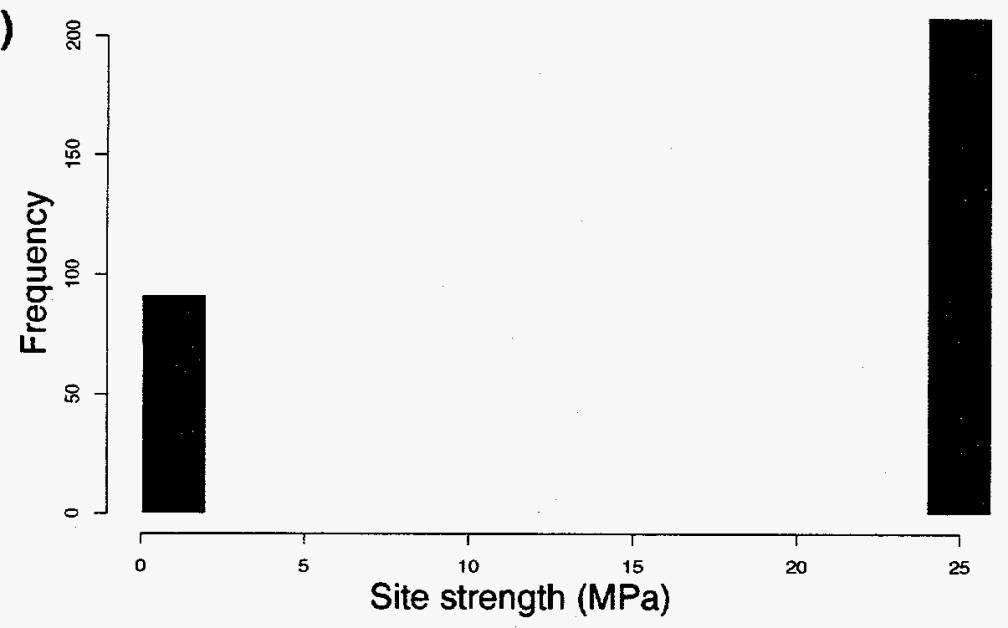

Figure $5.3-1$. Examples of different site strength distributions used in this study. a) $\mathrm{Tg}, \mathrm{i}$ in $35 \pm 34.5 \mathrm{MPa}, \mathrm{b}) \mathrm{Tg}, \mathrm{i}$ in $20 \pm 0.2 \mathrm{MPa}$ and c) Tg,i $2 \pm 0.2 \mathrm{MPa}$ or $26 \pm 0.26 \mathrm{MPa}$ 


\subsubsection{Homogeneous Site Strength}

Results for 10 trials computed with a homogeneous site strength distribution are presented in this section. A mean site strength of $20 \mathrm{MPa}$ was chosen because it is representative of the tensile strength of typical sandstones. Site strength values were picked randomly from a uniform distribution over $20 \pm 0.2 \mathrm{MPa} . m_{1}$ for these trials was in the range \pm 0.5 . Summary data for the 10 trials are presented in the Appendix. This set of values represents a material with uniform grain composition and cementation with some cracks and pores, which could be thought of as a well-sorted and well-cemented clean quartz arenite.

\section{Stress-Strain}

Stress-strain curves for these trials are shown in Figure 5.3-2. It is important to note that the values of stress and strain shown are those computed after each cracking event and are plotted sequentially. This figure shows nearly linear behavior up to a peak stress (or ultimate strength) for most trials, followed by strain-softening. This reflects the fact that as axial stress $\left(\sigma_{\mathrm{I}}\right)$ was applied, no cracks were formed until a critical stress level of approximately $40 \mathrm{MPa}$ was reached. Nonlinear behavior is shown after the peak stress and most of the trials show class I behavior with decreasing axial stress in the discretized region. Strain softening indicates that as cracks are produced, stress is transferred from the region covered with the lattice where cracking can occur, to the uncracked region of the side borders. For some of the trials stress remained constant while strain increased; for others stress decreased with strain, indicating strain-softening. Step increases for curves labeled 6 and 9 indicate that clusters of cracked sites that simulate cracks may form and extend, and then stop before forming a percolating or infinite cluster and failing the sample.

\section{Applied Stress vs Normalized Crack Strain Energy}

Applied stress vs normalized crack strain energy is shown in Figure 5.3-3. This plot shows that for these trials the normalized crack strain energy was in the range 0.35 to 0.65 when infinite or percolating clusters were formed and caused failure. This plot also shows that as 
stress is applied no crack strain energy develops until a critical stress level is reached; then for many of the trials all normalized crack strain energy is accumulated at one stress level. This indicates that for these trials, all sites break in rapid succession at one stress level. This is reasonable because all sites have similar strength and once an appropriate stress level is reached, all sites are near their breaking stress. When the weakest site breaks, stress is concentrated ahead of the crack tips, and as sites located ahead of the crack tips are already stressed to near their site strength, the increase due to the first cracked sites is sufficient to cause the cluster to propagate to the end of the array and form an infinite cluster.

\section{Overstress Ratio}

The overstress ratio defined in equation 4.40 can also be used to characterize the cracking of sites in the model. When a site $i$ satisfies the breaking rule given in equation 4.13 , the overstress ratio, $Q_{i}$, for the site can be plotted vs the number of sites that have previously broken. This technique has been used by de Arcangelis (1990) to study cracking in a lattice beam model with random site strength distributions. de Arcangelis (1990) used the overstress ratio plot to describe three regimes of fracture: an initial regime where cracks form randomly and their locations are controlled by the disorder in site strength, a regime of stable crack growth where the stress field controls breaking, and a catastrophic regime of unstable crack propagation where the system fails and overstress values can fluctuate widely.

The overstress ratio vs number of cracked sites for these trials is shown graphically in Figure 5.3-4. This figure shows the average overstress ratio (averaged over all 10 trials) for the first 45 cracked sites at $\sigma_{\mathrm{I}}=38 \mathrm{MPa}$. This plot shows that once one site breaks, the associated stress concentrations due to the crack create a higher overstress ratios at neighboring sites. The positive slope of this plot for the first 45 cracked sites in these trials shows that the clusters tend to propagate, and that the mean value of the overstress ratio for sites broken in these trials stays in a relatively narrow range of values between 1.0 and 2.2. This trend indicates that for these trials the crack growth is stable and controlled by the stress field in the simulation. 


\section{Number of Sites Broken vs Normalized Crack Strain Energy}

Another parameter that lends insight into the behavior is the normalized crack strain energy; the number of cracks vs normalized crack strain energy for these trials is plotted in Figure 5.3-5. This plot shows a dramatic change in slope after approximately 10 cracks are formed. This may indicate that crack interaction starts to dominate the behavior at this number of cracks. The relation between crack interaction and the strain energy plots is discussed in detail in Chapter 6. In general, the relationship of number of cracks and crack strain energy is quite linear. Large increases in strain energy with increasing number of cracks indicate crack extension, while increasing the number of cracked sites without increasing normalized crack strain energy indicates crack arrest. These concepts are discussed in more detail in Chapter 6.

\section{Crack Patterns}

Typical sequences showing the development of a crack in two of these trials are shown in Figures 5.3-6 $\mathrm{a}$ and $\mathrm{b}$. These figures show locations of cracked sites, and values of $\left(\sigma_{\phi, i}\right)_{S}$ at uncracked sites. The applied stress level is also shown. These figures show that no cracks form as axial stress, $\sigma_{\mathrm{I}}$, is applied until a critical stress level is reached and an initial crack is formed that propagates to each end of the array, forming a fracture. This is consistent with the stress-strain and applied stress vs normalized crack strain energy plots discussed above. This also shows that crack extension is in the direction parallel to the maximum principal stress.

Crack patterns at the percolation threshold are shown for all 10 trials in Figure 5.3-7. These plots show that most trials failed by the creation of one vertical or sub-vertical clusters of cracked sites. Moreover, for many of the trials the cracked sites form a thin zone that is often only one site wide. Other trials show a zone of cracked sites that may be a few sites wide. These patterns also show that most of the cracked sites are part of a single cluster, so that the cracked zone is surrounded by regions of uncracked sites. These macroscopic fracture surfaces are similar to those produced in tests on real rocks. This is analogous to a narrow crack or fracture zone splitting a rock sample. 


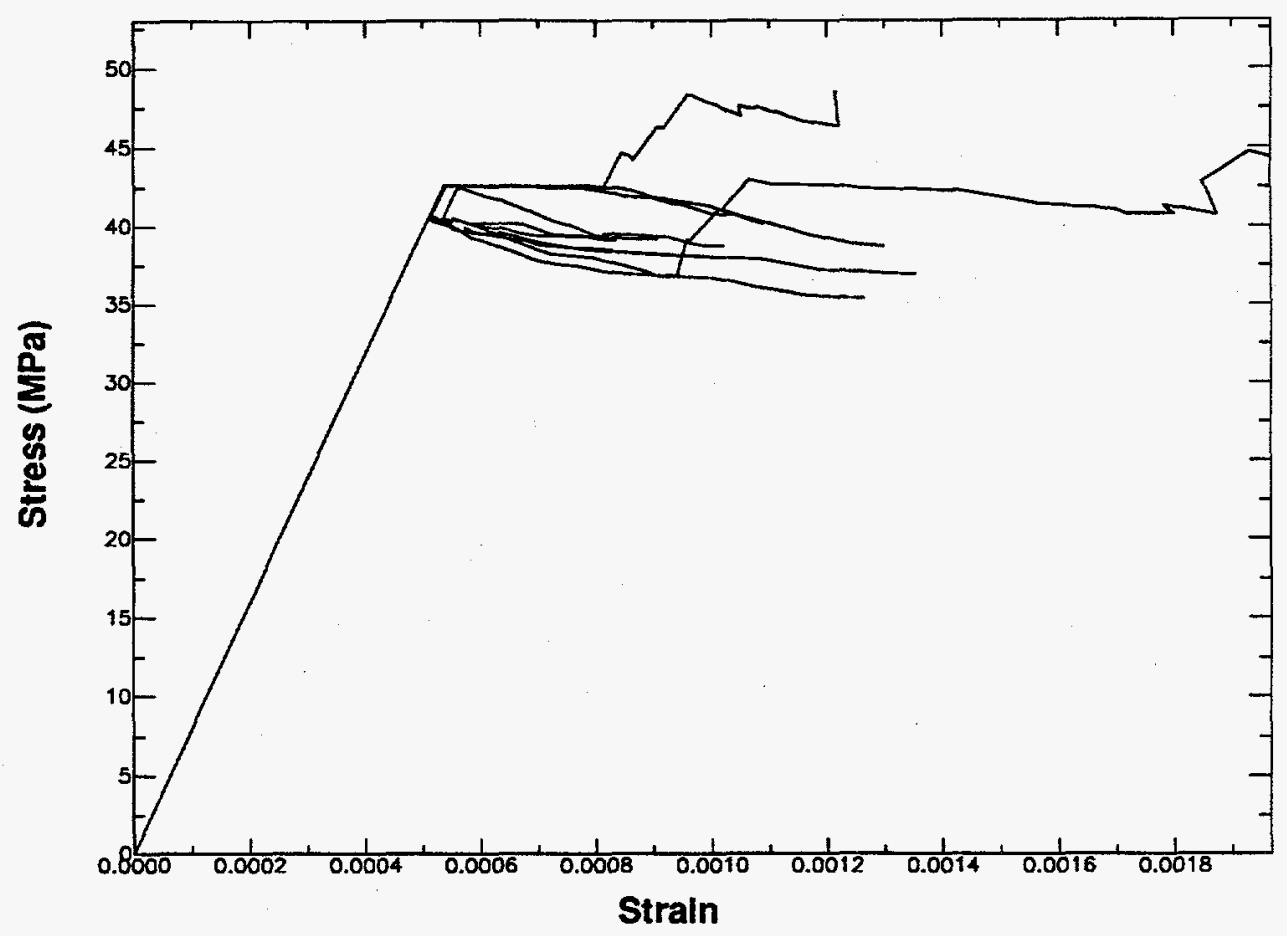

Figure 5.3 - 2 Stress strain curves for 10 trials computed using homogeneous site strength distributions.

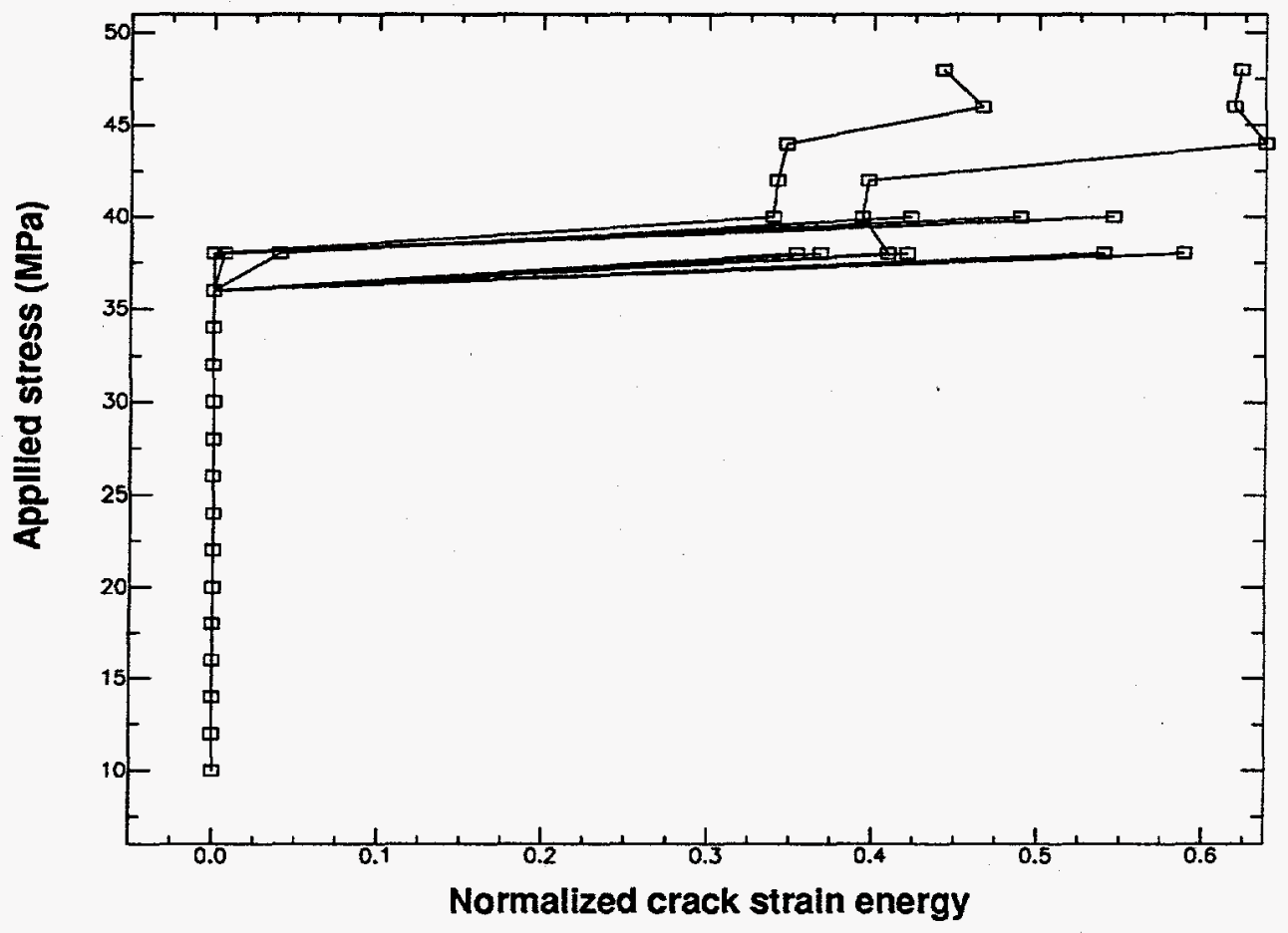

Figure 5.3 - 3 Relation of applied stress to normalized crack strain energy for 10 trials computed using homogeneous site strength distributions 


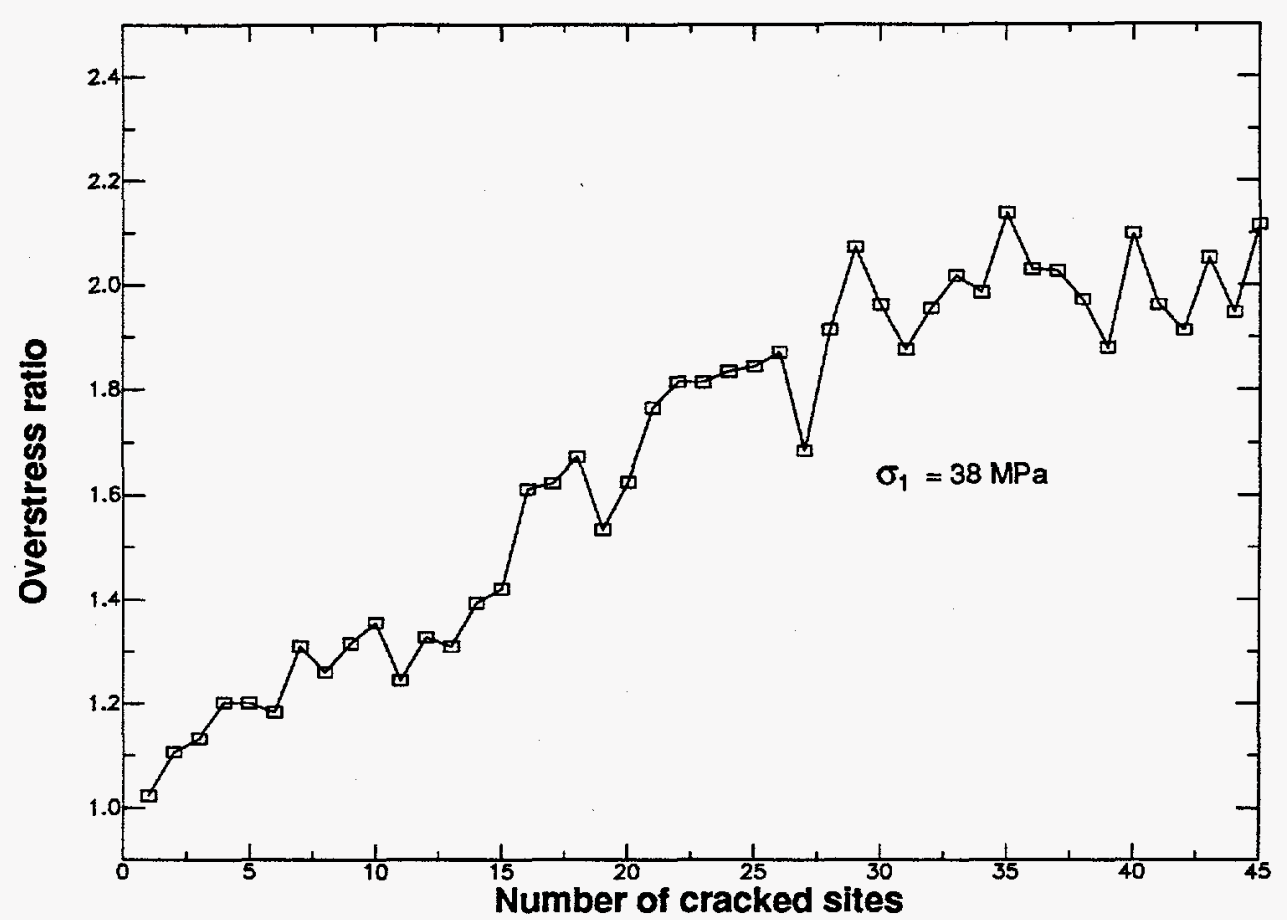

Figure 5.3 - 4 Overstress ratio versus number of cracked sites at an applied stress of $38 \mathrm{MPa}$ for homogeneous site strength distribution. Values represent averages taken over 10 trials.

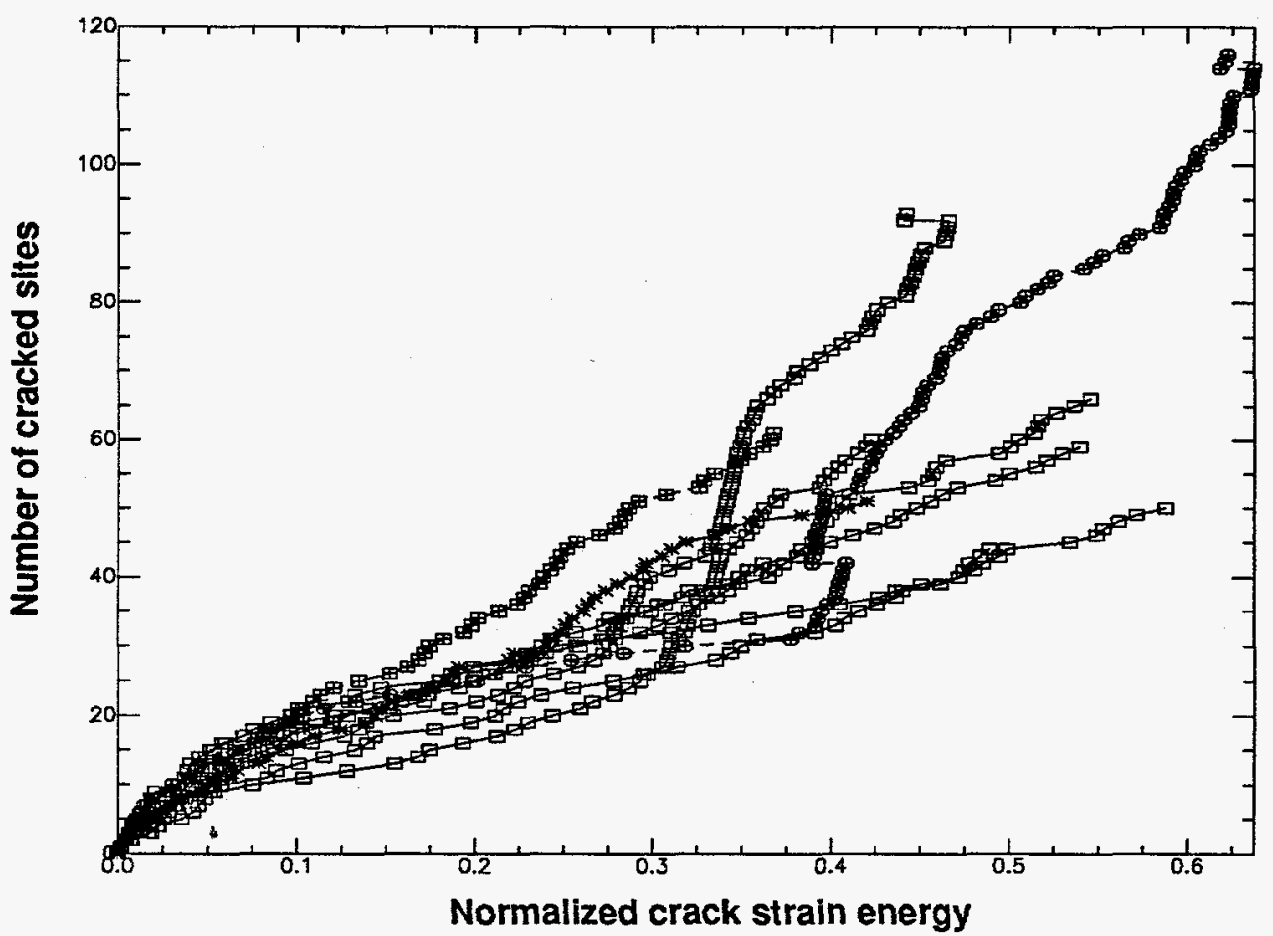

Figure 5.3 - 5 Relation of number of cracked sites to normalized crack strain energy for 10 trials computed using homogeneous site strength distributions. 

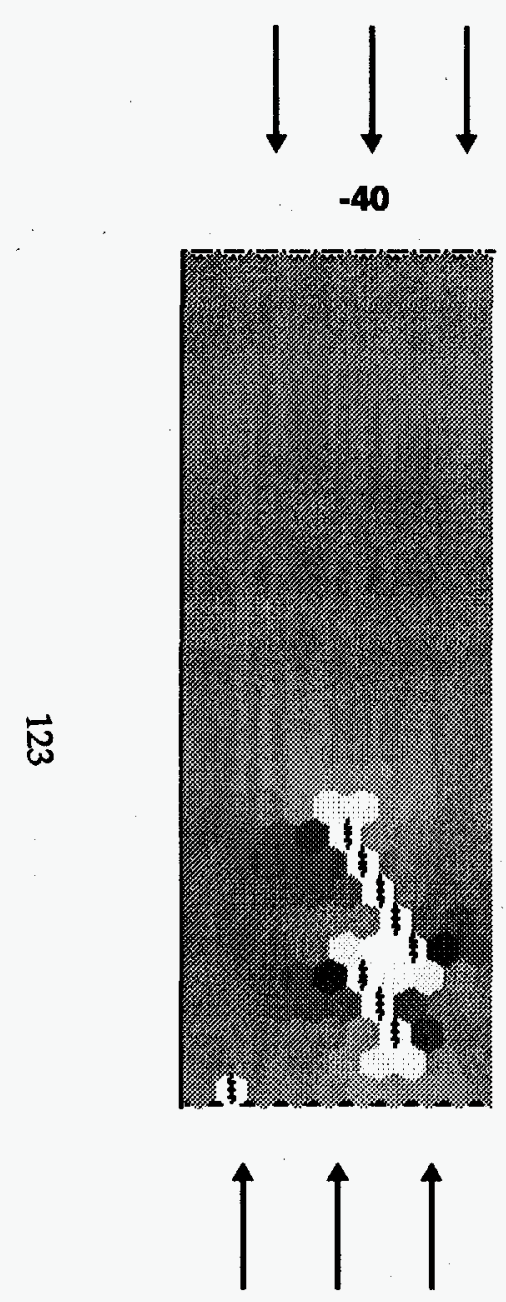
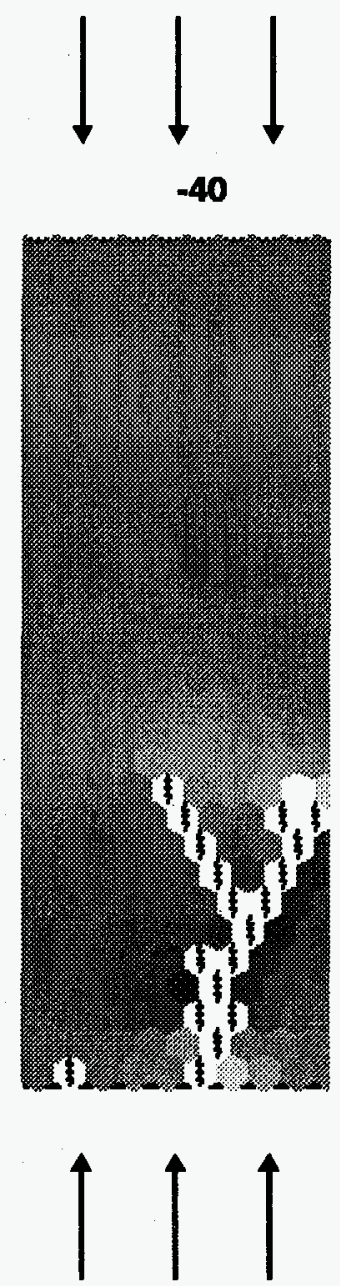
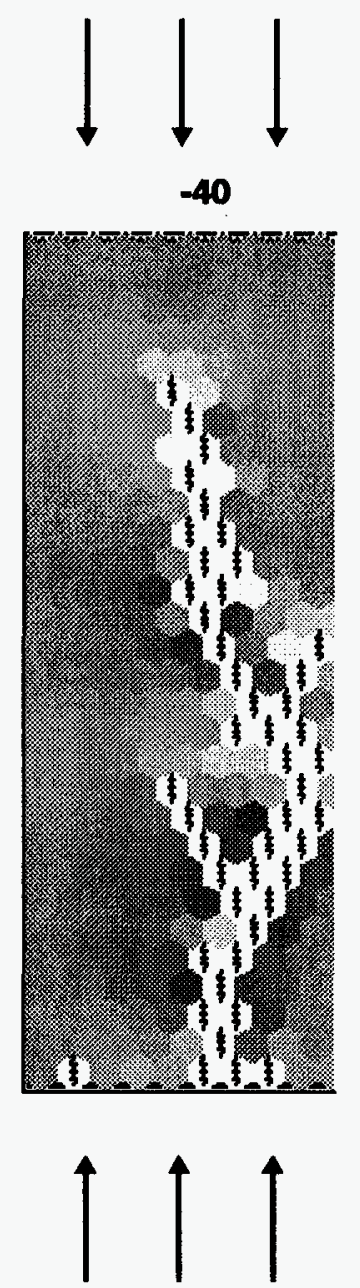
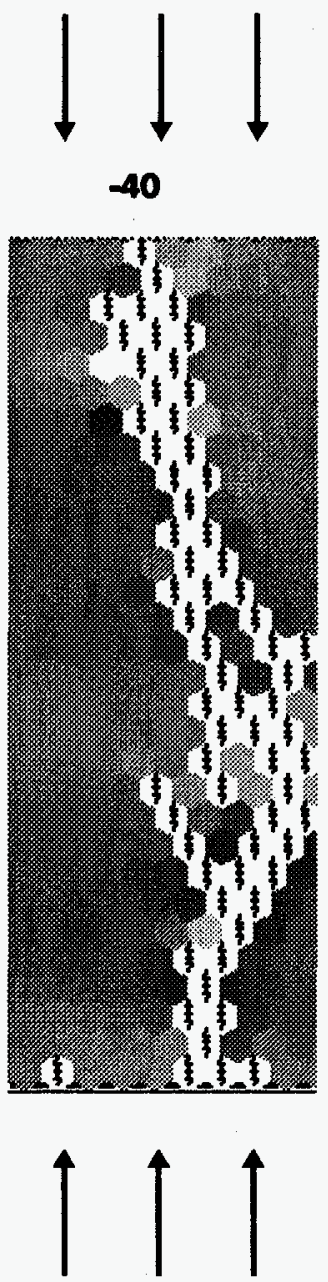

Tenslon

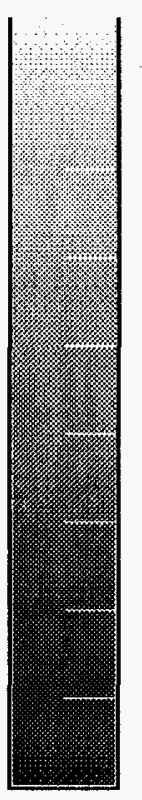

Compression

Figure 5.3-6. Crack pattern development for 2 trials with homogeneous site strength distributions. Shading shows horizontal stress in $S$ array. a) crack pattern for trial \#4 

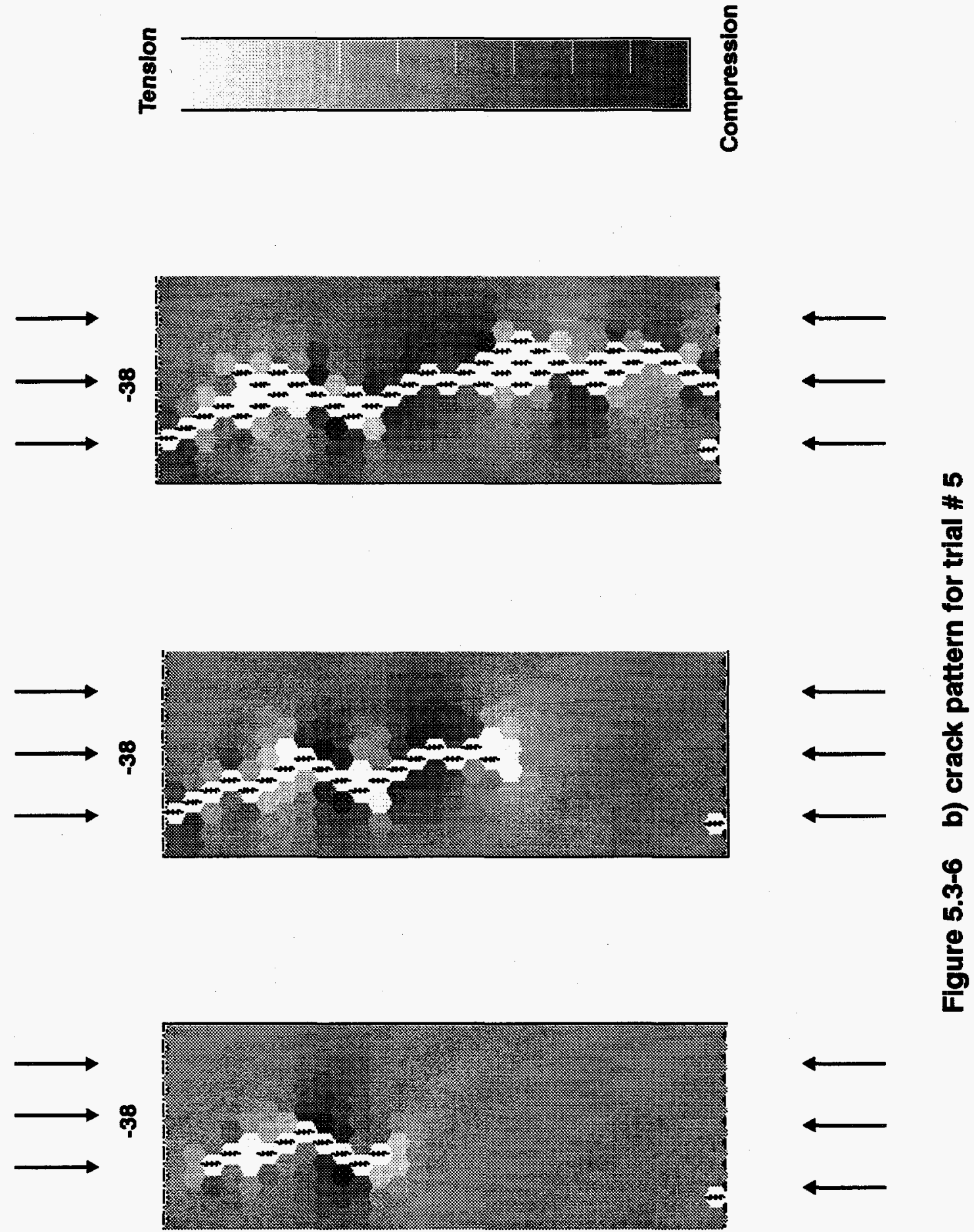

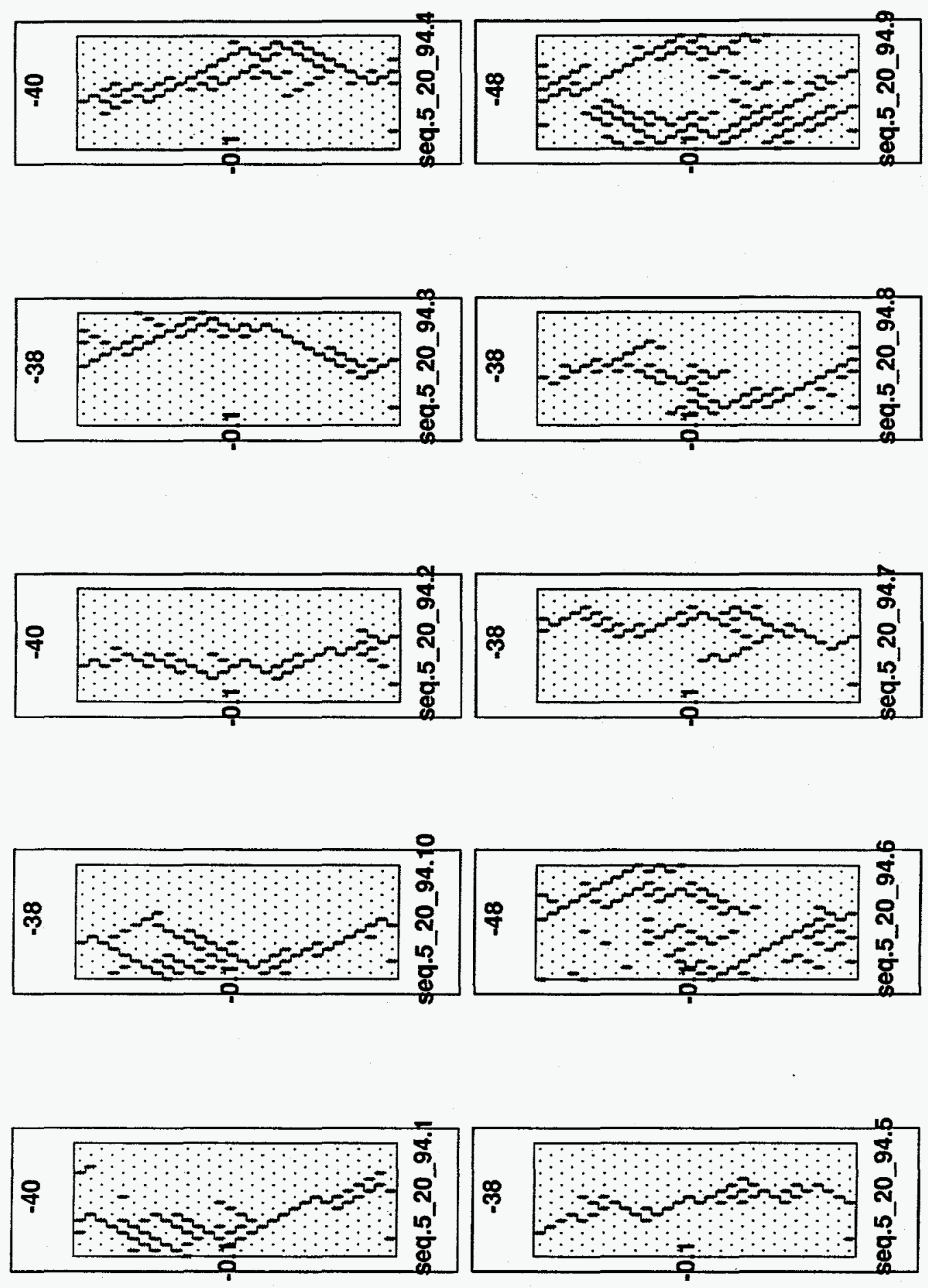

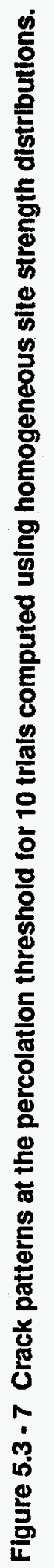




\section{Ultimate Strength and Percolation Threshold}

Mean ultimate strength (or peak strength) for these trials was $42.4 \pm 2.45 \mathrm{MPa}$, and the average percolation threshold for these trials was $p_{c}=0.18 \pm 0.03$, which is very low when compared with many percolation studies. However, these are small arrays with only 300 sites; thresholds for arrays with larger sizes are discussed in Section 5.3. The mean peak strength and percolation thresholds are discussed further in Section 5.3.4.

\subsubsection{Heterogeneous Site Strength}

Next, the case of uniaxial compression with $m_{1}$ in \pm 0.5 , but with heterogeneous site strength distribution, is considered. For these trials the mean site strength was $20 \mathrm{MPa}$, but the distribution was uniform over $20 \pm 19.9 \mathrm{MPa}$. For these trials the relative geometry of strong and weak sites and their spatial correlation with the $m_{1}$ values was the same as that used in the homogeneous site strength trials discussed above. ${ }^{1}$ The same data set for $m_{1}$ was used for each of these trials. Summary data for each of the trials is plotted in the Appendix.

\section{Stress-Strain}

Stress-strain curves for these trials are shown in Figure 5.3-8. All curves show nonlinearity at stress levels above $20 \mathrm{MPa}$, and the overall behavior is similar to that observed in rock. For these trials we observe that nonlinearity begins at applied $\sigma_{\mathrm{I}}$ values as low as $10 \mathrm{MPa}$, and significant nonlinearity occurs at axial stresses greater than $25 \mathrm{MPa}$. Several trials show sequences of strain-softening/strain-hardening/strain-softening as is sometimes observed in

\footnotetext{
1 This was done by using the same seed to produce both the homogeneous and heterogeneous site strength distributions for a given distribution of $m_{1}$ values. A site strength indicator, $T_{g, i}^{\prime}$, was defined for each site with values between \pm 1 . Ten data sets for $T_{g, i}^{\prime}$ were produced using a random number generator. Then, for the homogeneous site strength distribution the strengths were computed as $T_{g, i}=20+\left(0.2 * T_{g, i}^{\prime}\right)$. For the corresponding heterogeneous distribution trial $T_{g, i}$ was computed as $T_{g, i}=20+\left(19.9 * T_{g, i}^{\prime}\right)$.
} 
laboratory tests of rock samples, and strain-softening behavior is shown for all trials. They also show more variation in behavior than the curves for the homogeneous site strength distributions. These curves show the trend of strain at constant stress, but more strain-softening is observed, especially when $\sigma_{\mathrm{I}}$ is in the range $20-40 \mathrm{MPa}$. The overall amount of strain computed at the point when the critical cluster forms is similar to that for the homogeneous site strength distribution case. Moreover, the peak strength values are in the same range as for the homogeneous trial; this point is discussed further in Section 5.3.4. This figure shows that heterogeneous site strength distribution trials show a more gradual onset of deformation, starting at lower levels of applied stress.

\section{Applied Stress vs Normalized Crack Strain Energy}

Figure 5.3-9 plots the applied stress as a function of the normalized crack strain energy for these trials. This figure shows that crack strain energy is accumulated at all levels of applied stress and that at applied stresses of $24 \mathrm{MPa}$ and above, crack strain energy increases rapidly. This figure also shows that overall the values of normalized crack strain energy computed at formation of the infinite cluster (failure) were in the range 0.2 to 0.6 , which is similar to the range of this parameter for the trials with homogeneous site strength distribution. However, while the homogeneous site strength distribution trials showed that most of the crack strain energy was accumulated at one stress level (i.e., the failure stress), results for these trials show that a significant portion of the crack strain energy at failure may be accumulated at stress levels less than those associated with failure. Further, this figure shows that for this set of trials, cracks that form at stress levels less than $24 \mathrm{MPa}$ do not contribute significantly to the overall crack strain energy, and up to $50 \%$ of the crack strain energy observed at failure may be gained at stress levels above $50 \%$ of the failure stress.

\section{Overstress Ratio}

The behavior of the heterogeneous site strength distribution is further illustrated in Figure 5.3-10, which presents the overstress ratio vs number of broken sites at three different 
stress levels-10, 24, and $38 \mathrm{MPa}$. This figure shows behavior quite different than that for the homogeneous site strength distribution (see Figure 5.3-4). First, the range of values is much greater for the heterogeneous case and overstress ratios as high as 27 are observed. Second, the trends are much different, both between each of the stress levels and in comparison with the homogeneous site strength trials.

The values computed for $10 \mathrm{MPa}$ show the overstress ratio to decrease with increasing number of broken sites. The decreasing values reflect the fact that for this site strength distribution and at this stress level, heterogeneity in site strength controls the behavior. This is because at the onset of cracking the applied stress is low and the crack interaction forces are weak, so that the stress levels are similar at all of the sites and the weakest site breaks first, giving the highest possible overstress ratio. The next site to break must have a higher site strength, meaning the overstress ratio for that site must decrease.

At $24 \mathrm{MPa}$ the overstress values are lower and nearly constant, and this behavior is similar to that observed for the homogeneous site strength, except that the trend in these data is constant rather than increasing overstress with the number of cracks. In this regime the cracking of sites is determined by the applied stress field. Finally, the overstress ratio values for an applied stress of $38 \mathrm{MPa}$ show two types of behavior. Overstress values for the first 8 sites cracked at this stress level appear similar to those for stable crack growth, but for the next 12 sites the values fluctuate wildly over one order of magnitude from values of 2 to $26 \mathrm{MPa}$ before returning to a relatively constant value of near 2 for the last 16 sites to crack in this interval.

The general overstress behavior is decidedly different from that for the homogeneous site strength distribution, which shows one regime only. Moreover, this behavior is similar to that observed for the first stage of cracking in a lattice beam model with random site strength distributions by de Arcangelis (1990). 


\section{Number of Cracked Sites vs Normalized Crack Strain Energy}

The number of sites cracked is plotted vs normalized crack strain energy in Figure 5.3-11. This plot shows that for these trials the first 30 broken sites contribute very little to the crack strain energy. These sites crack at relatively low levels of applied stress and reflect the distribution of the weakest sites and/or the largest values of $m_{1}$. After approximately $10 \%$ of the sites are broken the slope of the curve in Figure 5.3-11 changes and may reflect increased crack interaction and increased loads. When this plot is viewed in conjunction with the plot of applied stress vs crack strain energy (Figure 5.3-9), it can be seen that the increase in normalized crack strain energy associated with stress levels between 25 and $30 \mathrm{MPa}$ is also associated with the accumulation of approximately 40 cracked sites. This may provide insight into how many cracks are necessary for crack interaction to dominate other parameters such as site strength distribution in the cracking behavior. This plot also shows sub-vertical segments where the number of cracks increases with little change in strain energy for some trials. This behavior may correspond to crack arrest and is discussed in detail in Chapter 6.

This shows much different behavior from the crack strain energy plot for the trials with homogeneous site strength distribution discussed earlier.

\section{Crack Patterns}

Figures 5.3-12 a and $\mathrm{b}$ show patterns of cracked sites for trials 4 and 10 at different stages of the cluster development, and illustrate how the cluster structure developed for typical trials of this set. These figures show that at first cracked sites are randomly distributed. Subsequent to this clusters form and then link to form an infinite cluster and cause failure. This is fundamentally different from the behavior observed in the homogeneous case and will be discussed in more detail in Chapter 6.

Patterns of cracked sites for the 10 trials at the percolation threshold are shown in Figure 5.3-13. This figure shows that for these trials the patterns are generally similar to those for the narrow site strength distribution but that more en-echelon patterns are observed 


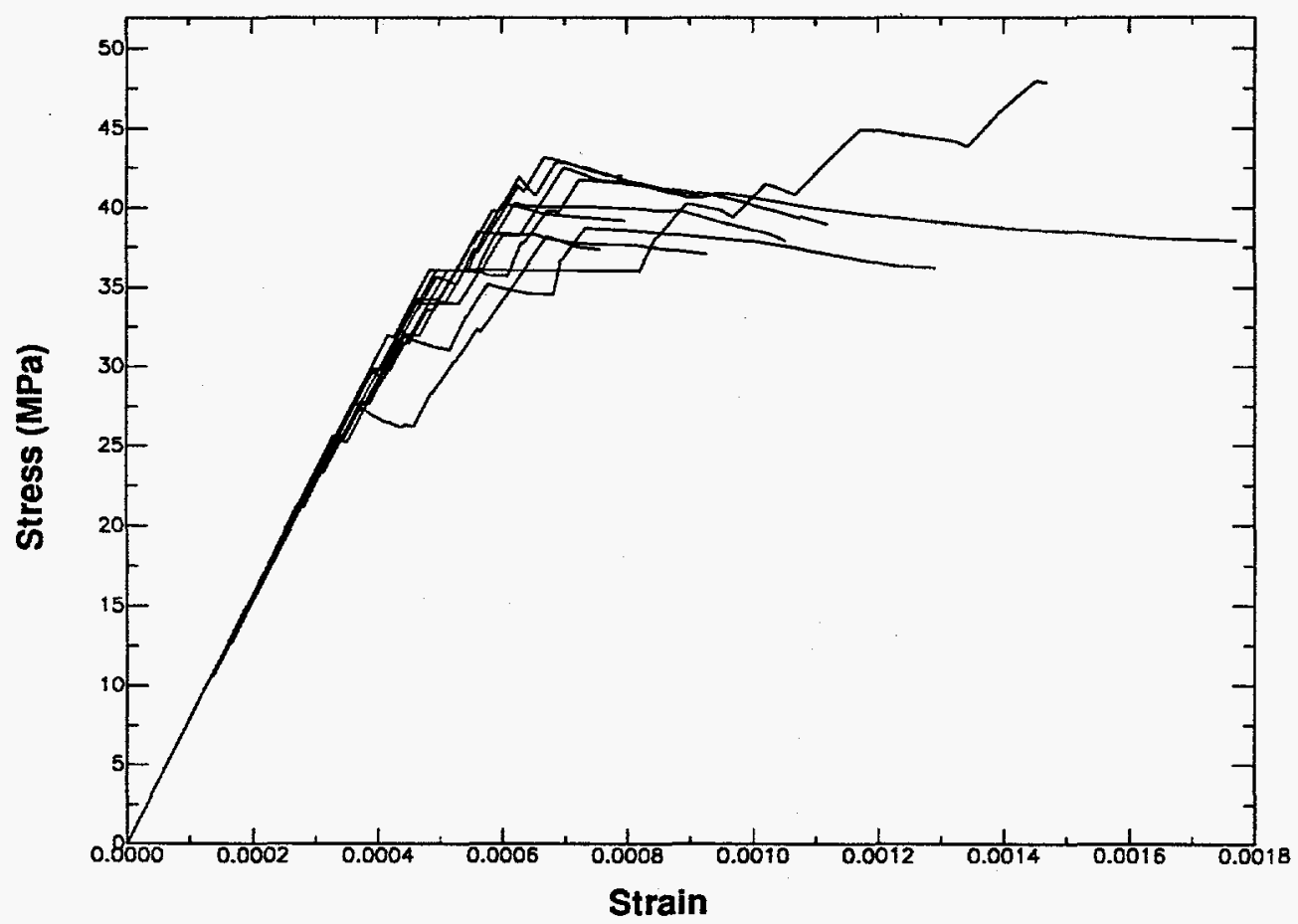

Figure 5.3 - 8 Stress-strain curves for 10 trials computed using heterogeneous site strength distributions.

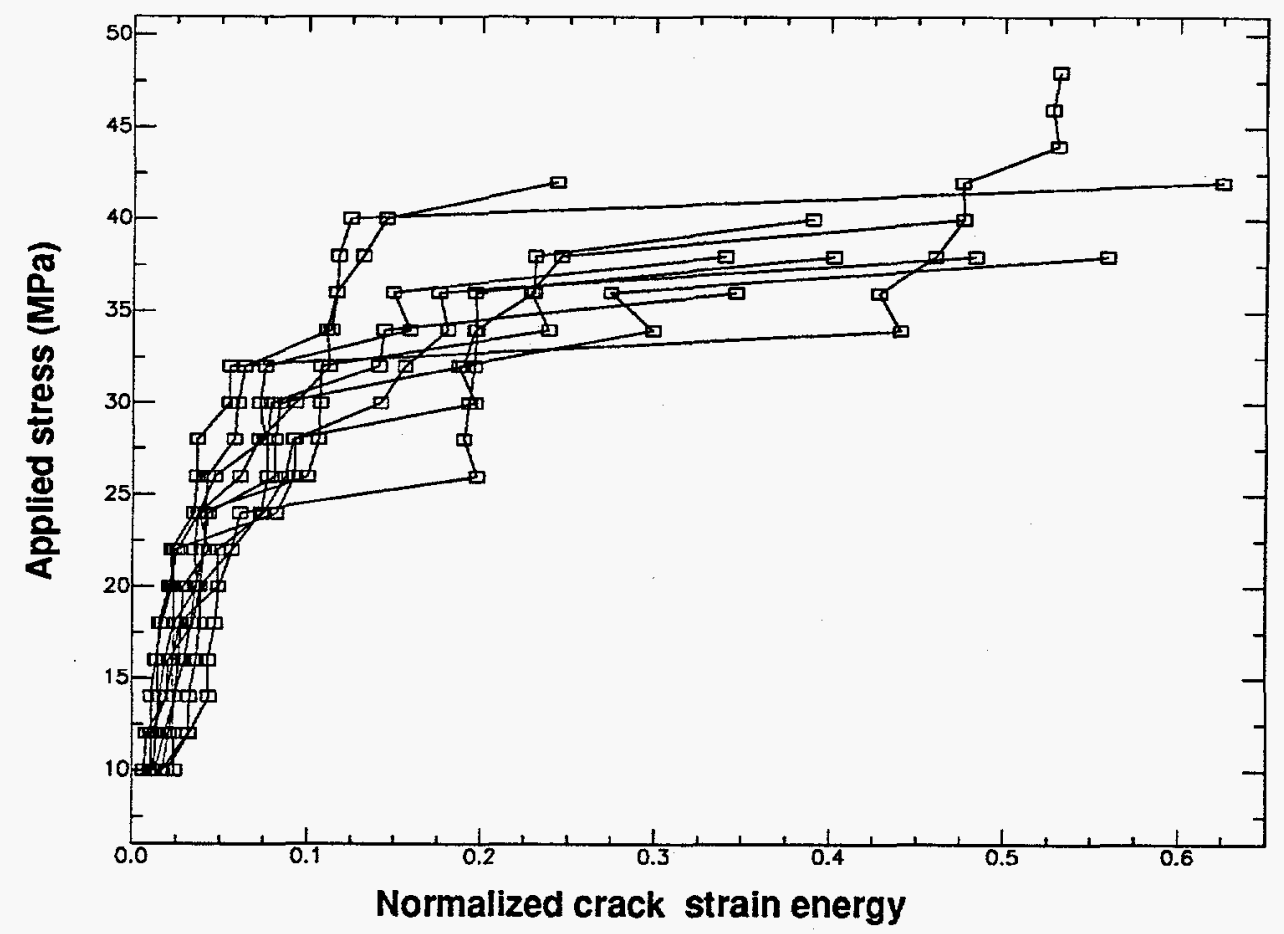

Figure 5.3 - 9 Relation of applied stress to normalized crack strain energy for 10 trials computed using heterogeneous site strength distributions. 


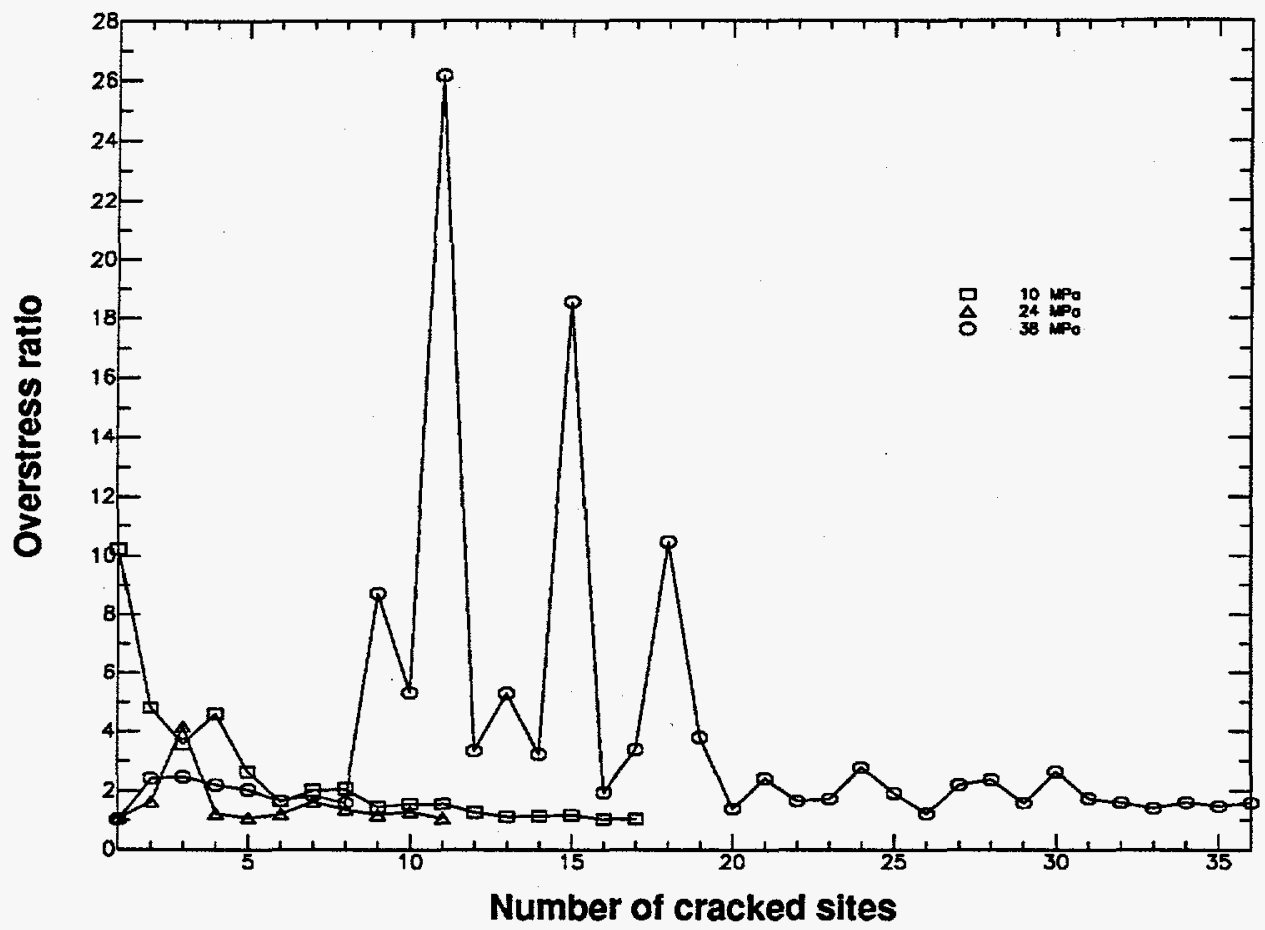

Figure 5.3 - 10 Relation of overstress ratio to number of cracked sites at three different stress levels, for heterogeneous site strength distributions. Values are averaged over 10 trials.

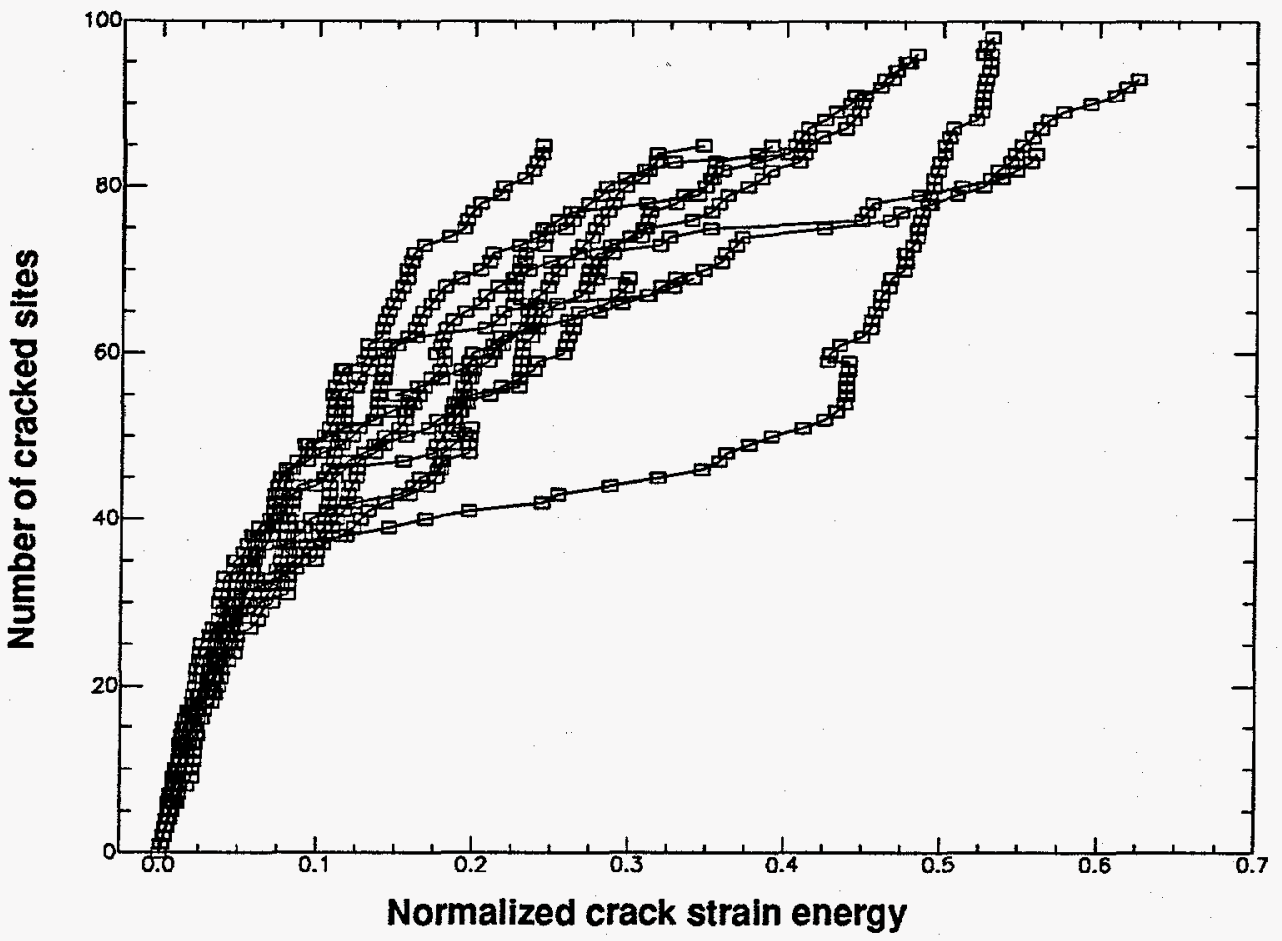

Figure 5.3 - 11 Relation of number of cracked sites to normalized crack strain energy for 10 trials computed using heterogeneous site strength distributions. 


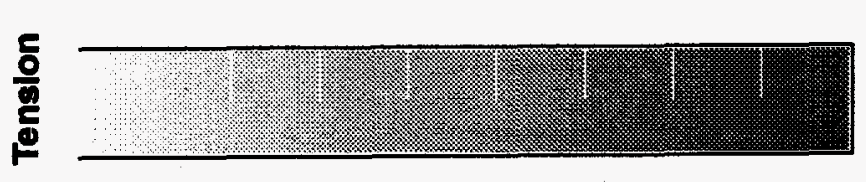

$\frac{5}{0}$
$\frac{8}{8}$
응
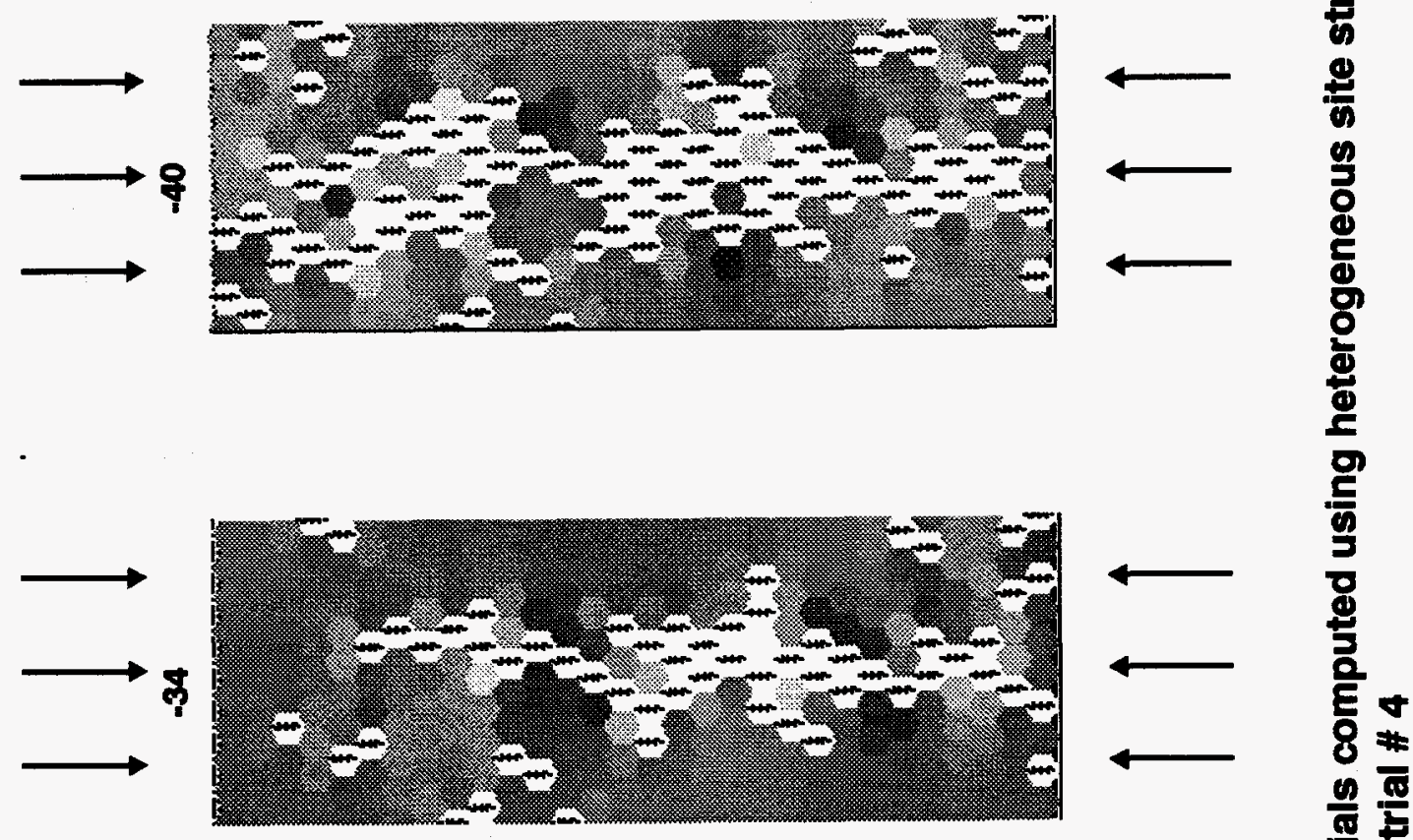

돈

เ

흔
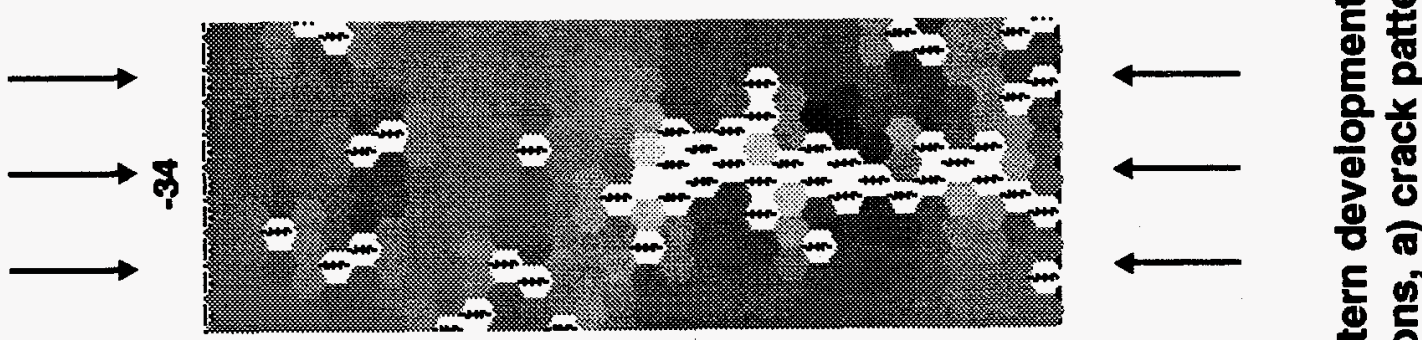

응 홍

$\leftarrow$

ชิ

동 옹
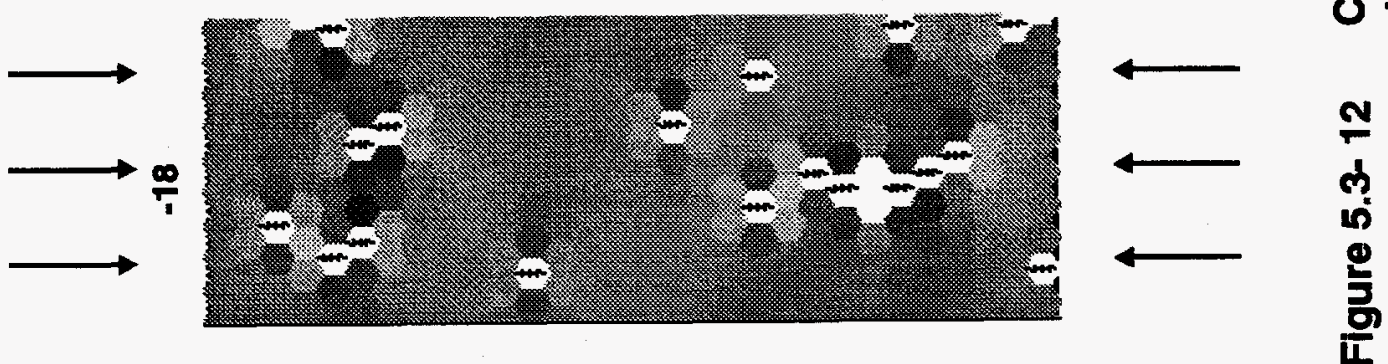

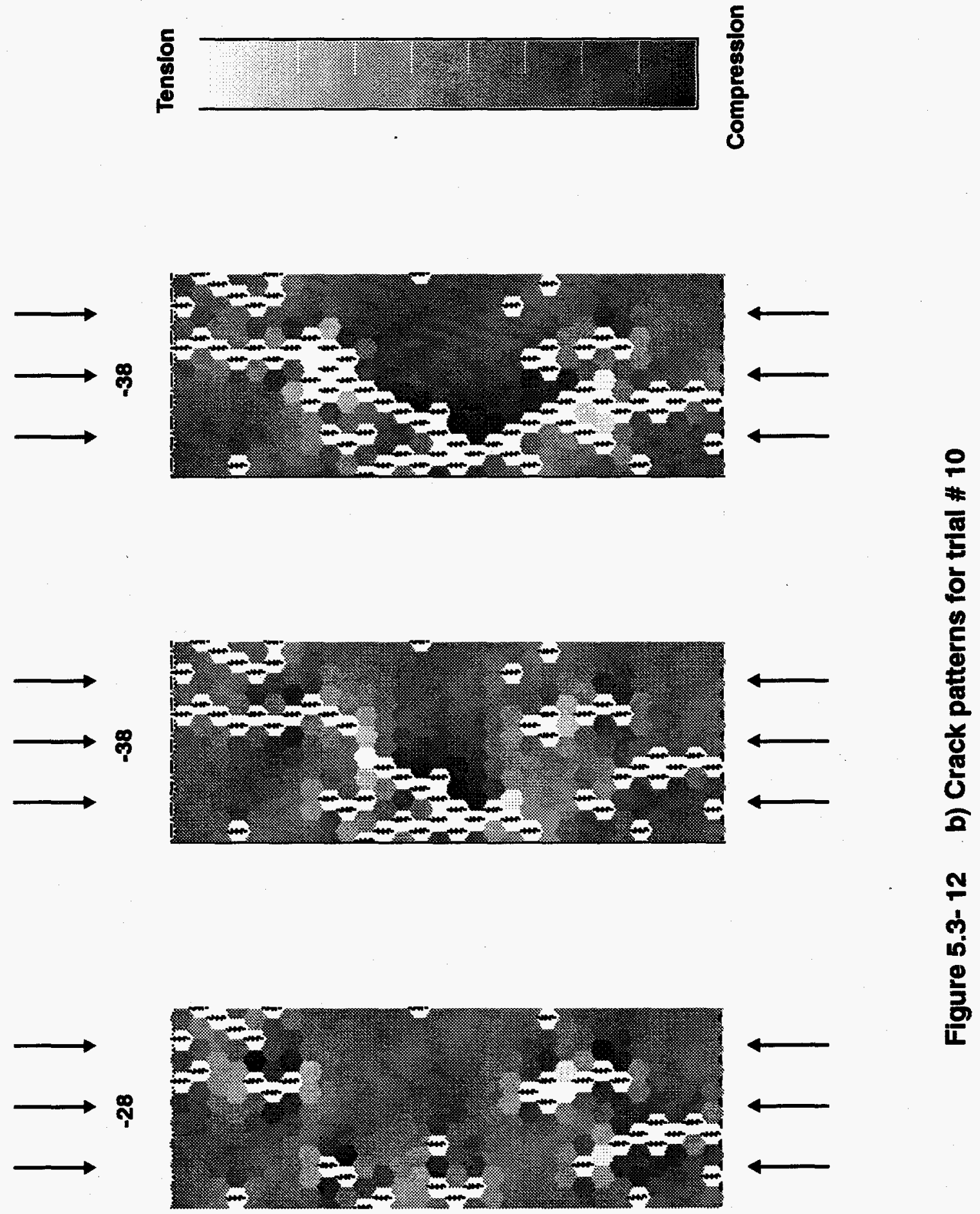

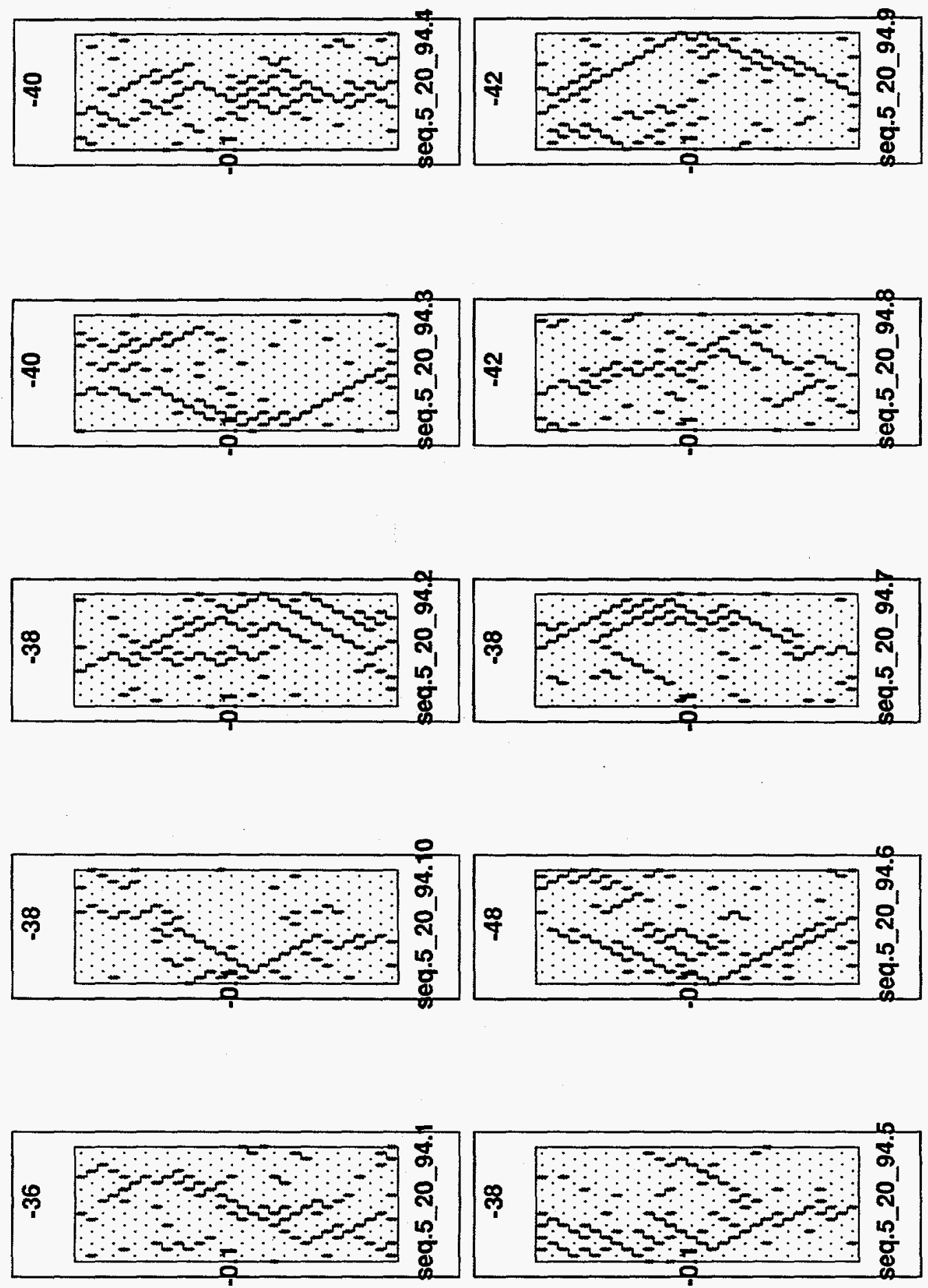
and the cracked sites are more widely distributed. Again, the macroscopic surfaces formed by the major clusters of cracked sites are similar in cross section to fractures formed in rock specimens tested in compression. Moreover, there is more stepping of clusters to the side and there are more small clusters of cracked sites that are unconnected to the percolating cluster. These cracked sites in the material away from the main clusters serve to reduce the effective modulus of the material, as is reflected in the stress-strain behavior. Although the trials have the same relative geometry for strong and weak sites as the corresponding homogeneous site strength trial, and identical spatial distributions of the $m_{1}$ parameter, the patterns of cracked sites for the homogeneous and heterogeneous site strength distributions are similar for only some of the trials (e.g., 10, 9, 6, 4). In some cases the percolating cluster is narrower than for the corresponding trial in the homogeneous site strength trials (e.g., trials 10, 1, 6, 7, 8), while in other trials the percolating cluster is wider (e.g., trials 2,3 ).

\section{Ultimate Stress and Percolation Threshold}

The average value of the peak or ultimate strength for the heterogeneous trials discussed above is $41.4 \pm 2.78 \mathrm{MPa}$ (see Appendix). This is similar to the $42.4 \mathrm{MPa}$ observed for the trials with homogeneous site strength distribution, and may indicate that when site strength is uniformly distributed over an interval, the mean site strength controls the peak strength. This is discussed further in Section 5.3.5. Percolation thresholds for these trials had a mean of $0.29 \pm$ 0.03 , which is higher than for the homogeneous site strength trials. For heterogeneous site strength distributions more cracks form at lower stress.

In summary, the heterogeneous site strength distribution trials show more strain-softening, similar peak strength, higher percolation threshold, and more realistic stress-strain behavior than the homogeneous site strength trials. Cracking of sites occurs at much lower levels of applied stress, but these sites do not interact until approximately $10 \%$ of the total number of sites are broken and do not affect the predicted peak strength. 


\subsubsection{Bimodal Site Strength Distribution}

This section presents results of a set of trials computed using a bimodal distribution of site strengths. The purpose of using a bimodal site strength distribution was to determine if the presence of a population of very weak sites would influence the macroscopic behavior. The bimodal distribution of site strengths used had $25 \%$ of the sites assigned a tensile strength of $2 \pm 0.2 \mathrm{MPa}$ and $75 \%$ of the sites assigned a tensile strength of $26 \pm 0.26 \mathrm{MPa}$. This distribution is illustrated in Figure 5.3-1 $\mathrm{f}$. The mean value of site strength for the array was $20 \mathrm{MPa}$. A series of 10 uniaxial trials were conducted with $m_{1}$ in \pm 0.5 . Summary data for these 10 trials are presented in the Appendix.

\section{Stress-Strain}

Stress-strain curves for these 10 trials are shown in Figure 5.3-14. This plot shows that the behavior of all of the 10 trials was similar except for one trial that required a much higher stress to reach fracture. The values of ultimate strength and the stress-strain behavior are similar to those observed for the homogeneous and heterogeneous site strength cases discussed previously. Some of the individual curves show stress-strain behavior similar to that observed for the heterogeneous site strength case, and nonlinearity is observed at axial stresses above $10 \mathrm{MPa}$. However, the general behavior is more like that observed for the homogeneous site strength trials, as the strain-softening/strain-hardening/strain-softening behavior is often absent or is less pronounced. The one trial that shows much higher peak stress and strain behaved similar to the other trials, but may have been subject to crack arrest.

\section{Applied Stress vs Normalized Crack Strain Energy}

Applied stress is plotted vs normalized crack strain energy in Figure 5.3-15. This plot shows that the overall range of normalized crack strain energy is similar to that observed for the heterogeneous and homogeneous site strength cases, that applying the initial stress of $10 \mathrm{MPa}$ created initial crack strain energy in all of the trials, and that as applied stress was raised from $10 \mathrm{MPa}$ to $30 \mathrm{MPa}$ very little new crack strain energy formed in any of the trials. At 
applied stress levels above $30 \mathrm{MPa}$ behavior changed dramatically; many of the trials reached a critical stress level at which the clusters of cracked sites propagated and formed infinite or

percolating clusters. Additional insight into the behavior can be gained from Figure 5.3-16, which shows the number of cracks vs the applied stress for the 10 trials. This figure shows that most of the population of weak sites cracked at stresses below $20 \mathrm{MPa}$ and that very few sites were cracked at stress levels between 20 and $30 \mathrm{MPa}$. This indicates that although the population of weak sites did break, the stress levels were inadequate to drive the crack interaction forces, and these cracked sites had little effect on the behavior. Moreover, breaking the weak sites left a material with homogeneous site strength distribution, but with a randomly located population of cracked sites. When the applied stress was increased to a sufficient level, crack interaction stresses due to the these cracked sites began to dominate the site strengths, and some small, favorably oriented clusters propagated as if in a material with homogeneous site strength distribution. In addition, due to the different geometries of the clusters, this propagation occurred at different stress levels, and produced a wide range of strengths. This explains the observation that the overall character of the stress-strain and applied stress vs crack strain energy curves is similar to that for the homogeneous site strength case, while the range of peak stresses is much broader.

\section{Overstress Ratio}

Average overstress values for these trials at applied stresses of 10 and $38 \mathrm{MPa}$ are shown in Figure 5.3-17. The values for cracks formed at $10 \mathrm{MPa}$ show a gradual decline in overstress, with maximum value less than 3 . This reflects that the disorder in the site strength is controlling the cracking of sites, and the smooth nature of the decline is due to the narrow width of the site strength distribution for the weak sites. In contrast, the overstress ratios for an applied stress of $38 \mathrm{MPa}$ are indicative of catastrophic behavior, with wildly varying values that range as high as 19 . The values for $38 \mathrm{MPa}$ are similar to those observed for the heterogeneous site strength case at this stress level. 


\section{Crack Patterns}

Figures 5.3-18 a and $b$ show the sequence of site cracking for trials 1 and 6 . These illustrate many of the points discussed above. In particular, there is a population of randomly distributed cracks that have formed at an applied stress of $10 \mathrm{MPa}$ and represent the weakest sites. Then as stress is increased, a stress level sufficient to form a critical cluster is reached and one cluster runs away.

Patterns of cracked sites at failure for the 10 trials are shown in Figure 5.3-19. In general, these patterns are very similar to those observed for the heterogeneous site strength trials. However, in three of the trials the percolating cluster includes two branches that connect to either the top or the bottom. This is shown clearly in trials 3 and 5, but also occurs in trial 8 . This phenomenon was not observed in the homogeneous or heterogeneous trials.

\section{Ultimate Strength and Percolation Threshold}

The average ultimate stress for these trials was $48.5 \mathrm{MPa}$ (Table 5.3-3), which is higher than for the trials with $T_{g} 20 \pm .2$ and $T_{g} 20 \pm 19.9$, and was lower than strength for the $T_{g} 26 \pm .26$. This indicates that while many of the weak sites broke at low levels of applied stress, the overall strength was increased due to the majority $(75 \%)$ of the sites having mean strength of $26 \mathrm{MPa}$, which is higher than the mean of $20 \mathrm{MPa}$ for the entire array. However, the weak sites in the bimodal distribution had some effect, as the peak strength of the bimodal trials was lower than the peak strength for trials with strength distribution $T_{g} 26 \pm .26$ (see Section 5.3.4). Values computed without highest trial are peak strength $=42.9 \pm 4.25 \mathrm{MPa}$ and percolation threshold $=0.29 \pm 0.03$.

The bimodal distribution provides a certain percentage of weak/strong sites. One question is: how many weak/strong sites need to be included to alter the macroscopic behavior? This may depend on the radius of influence of the sites cracked. At low stresses the radius of influence for each cracked site is small; thus the fraction of weak sites necessary to cause fracture may be near the value of the percolation threshold for random processes. Note that 


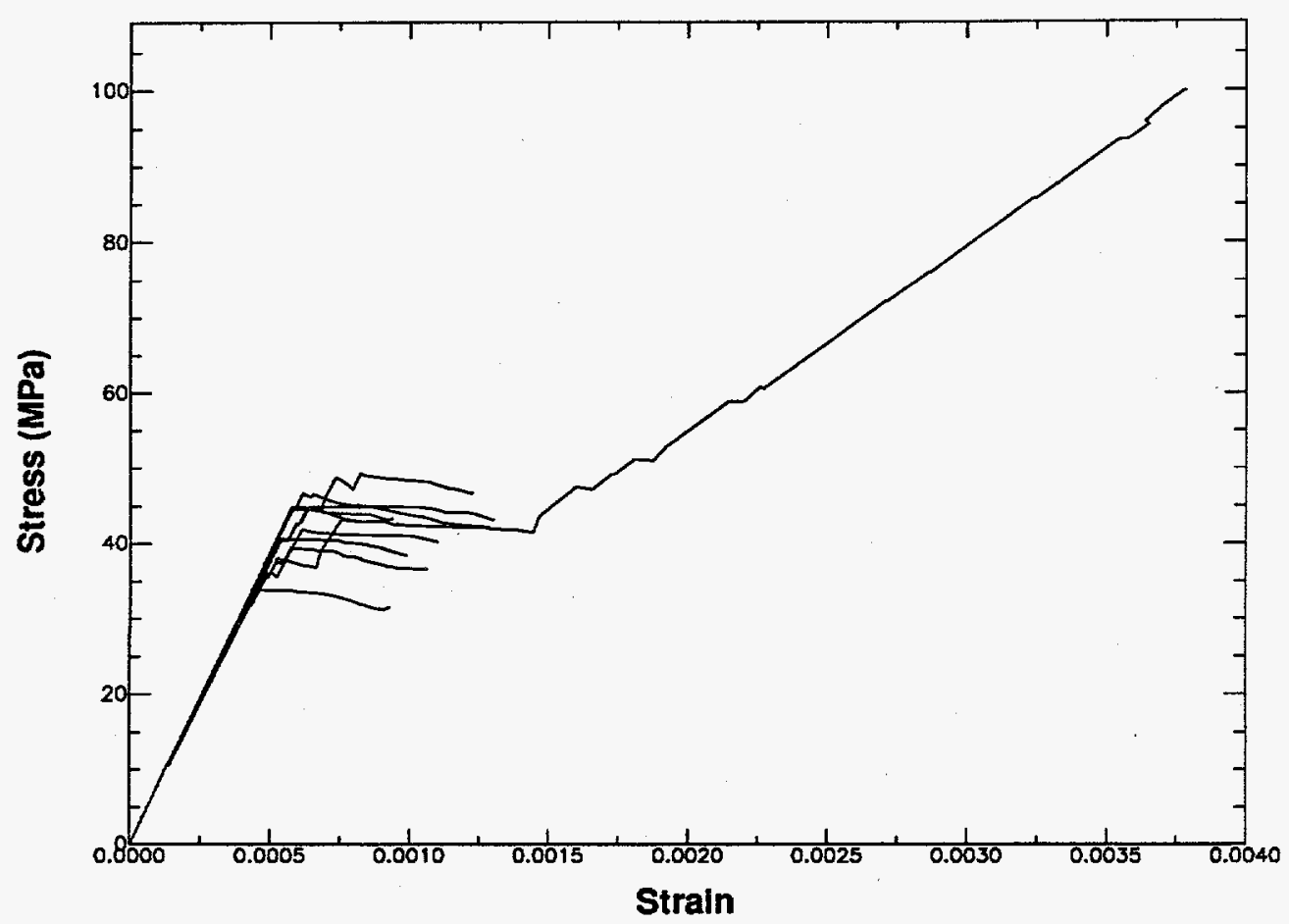

Figure 5.3 - 14 Stress-strain curves for 10 trials computed using bimodal site strength distributions.

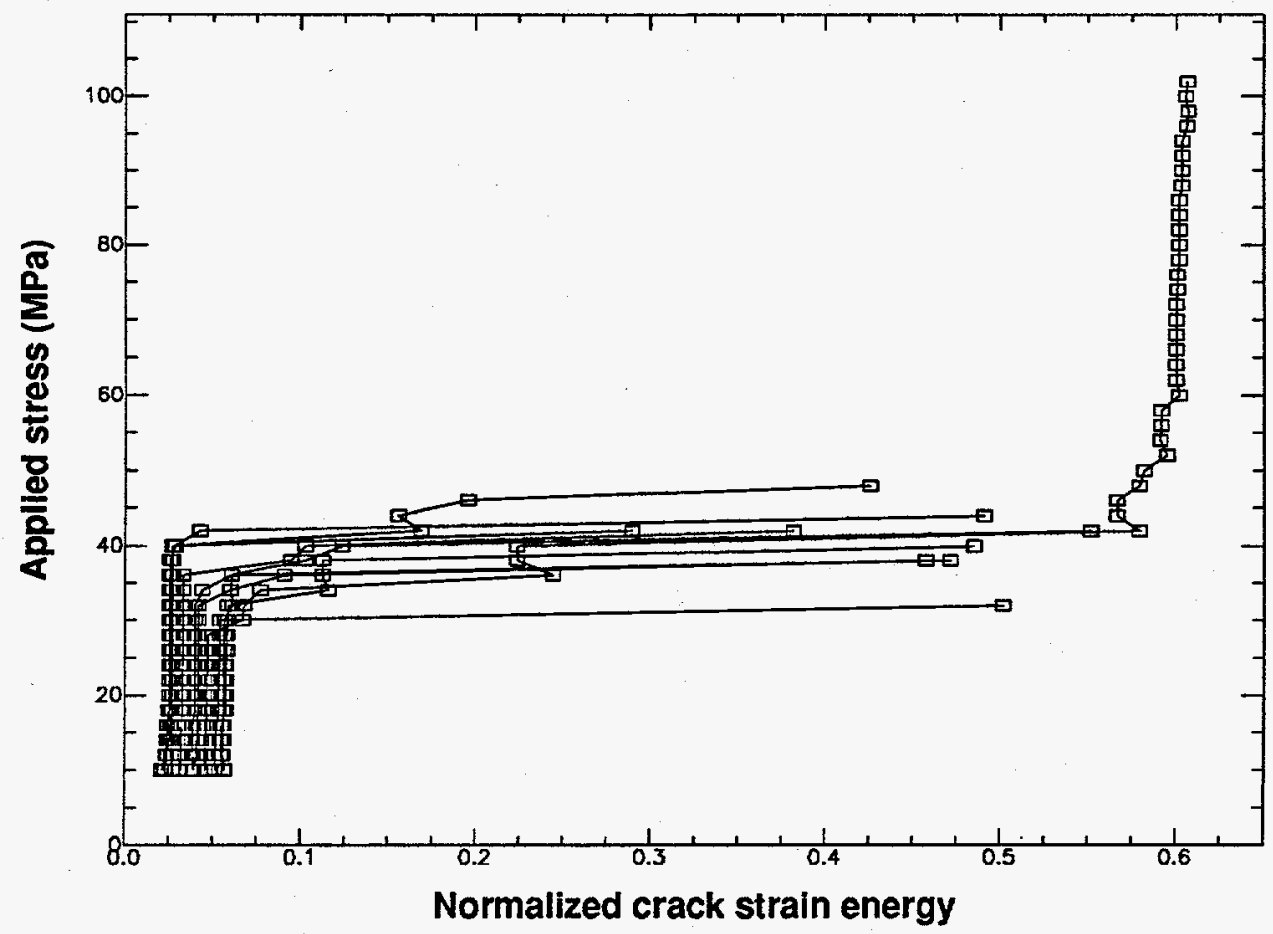

Figure 5.3 - 15 Relation of applied stress to normalized crack strain energy for 10 trials computed using bimodal site strength distributions. 


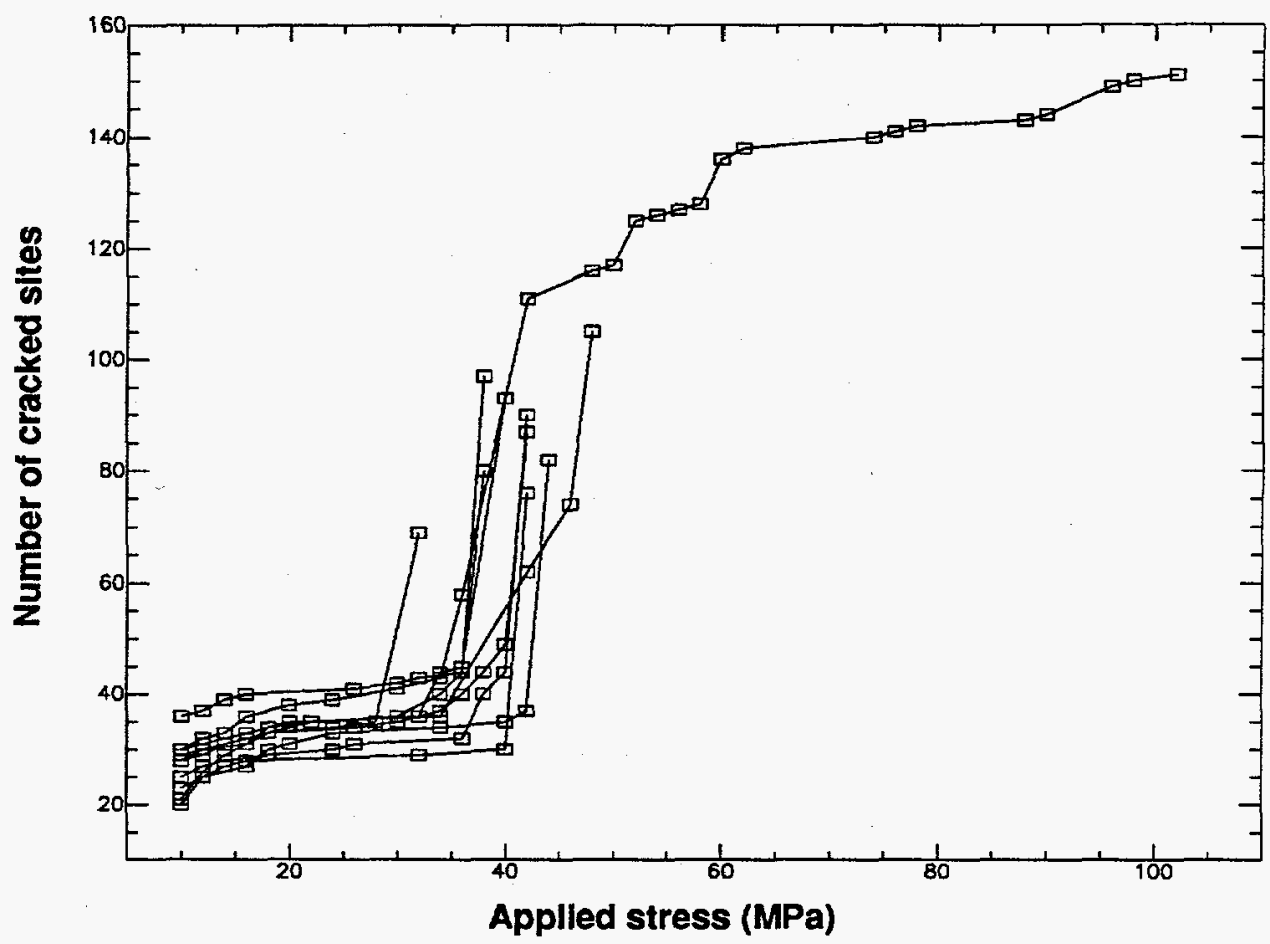

Figure 5.3 - 16 Relation of number of cracked sites to applied stress for 10 trials computed using bimodal site strength distributions.

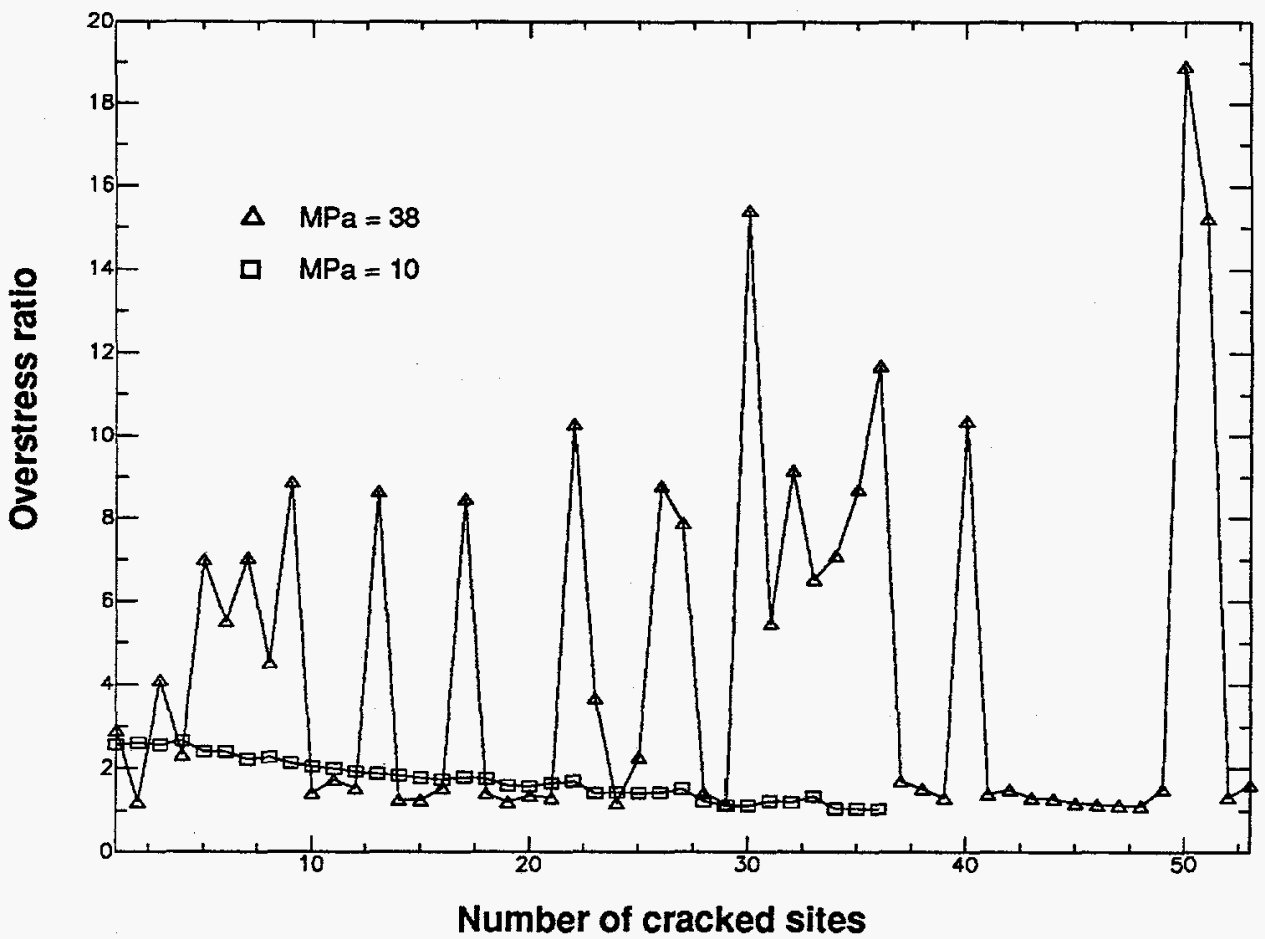

Figure 5.3 -17 Relation of overstress ratio to number of cracked sites at 2 levels of applied stress, for trials computed using bimodal site strength distributions. Values are averaged over 10 trials. 

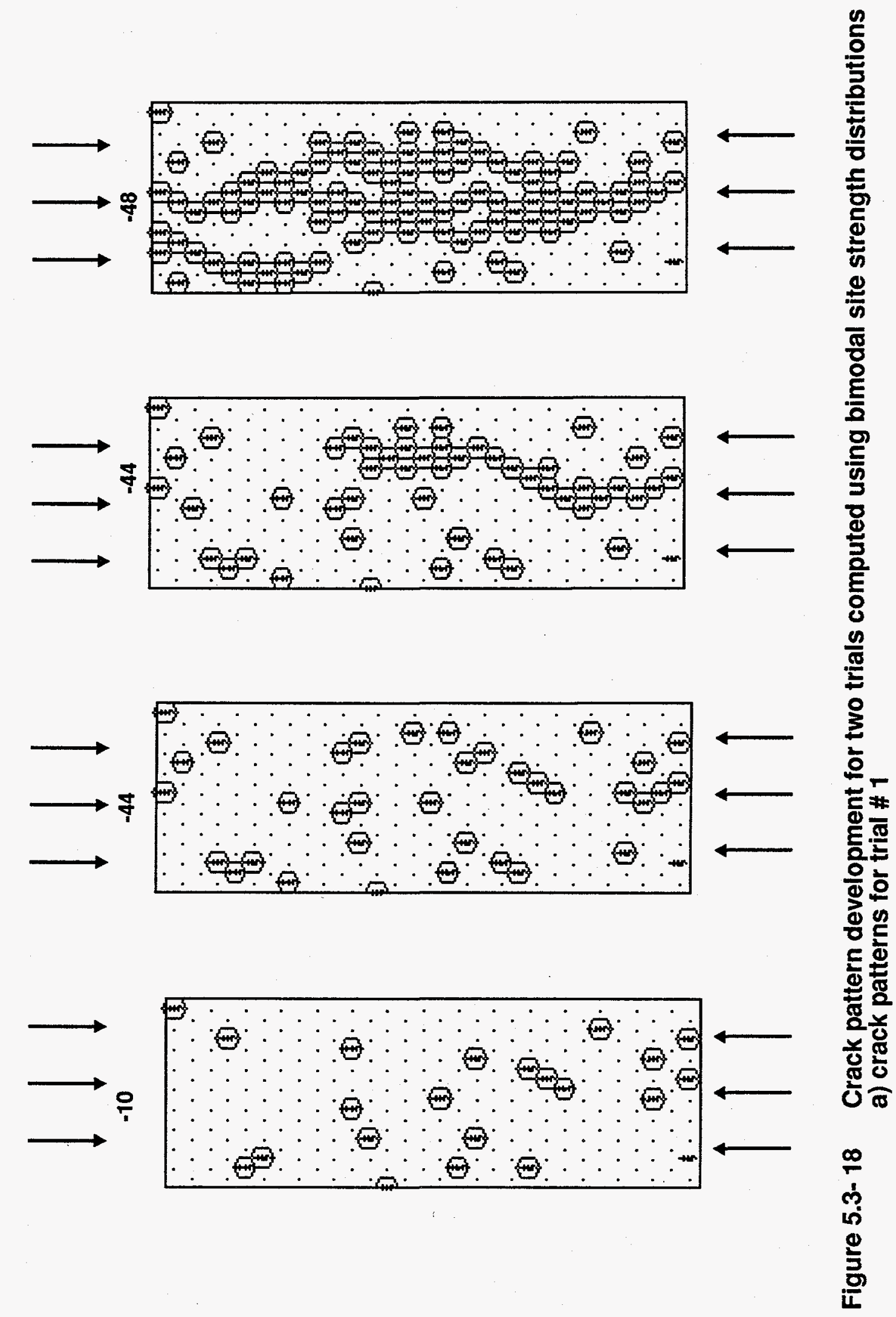

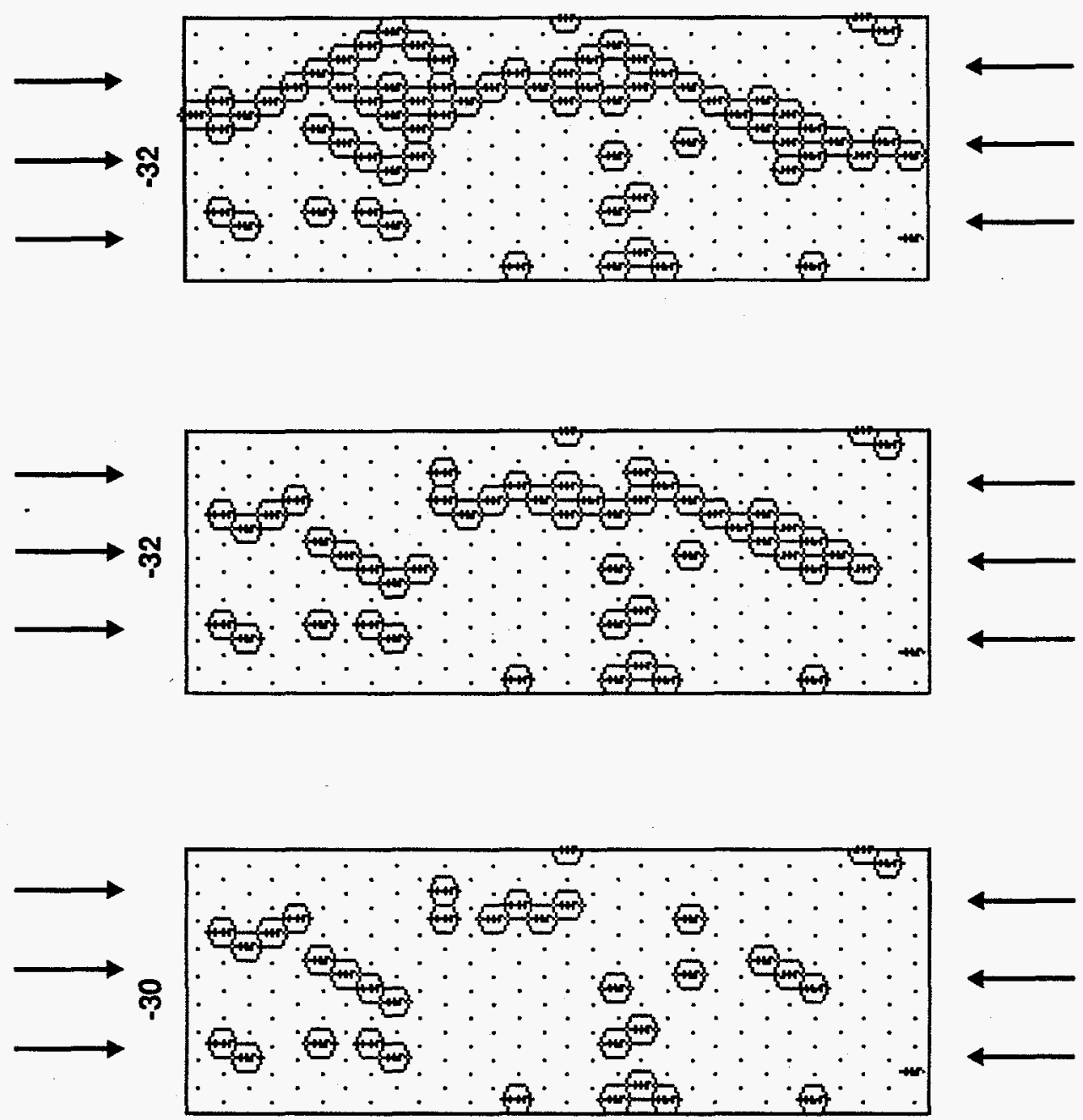

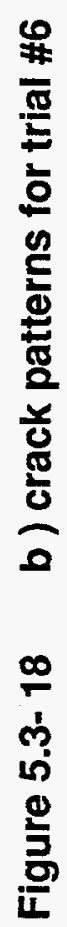

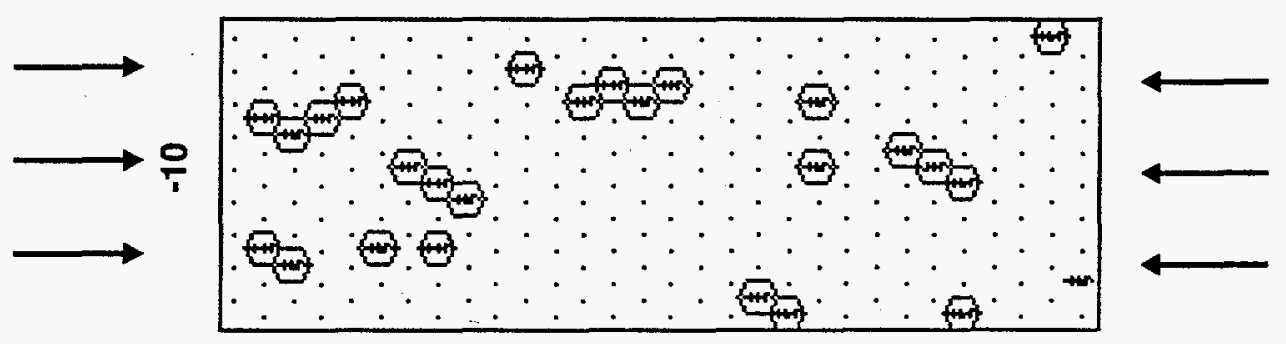



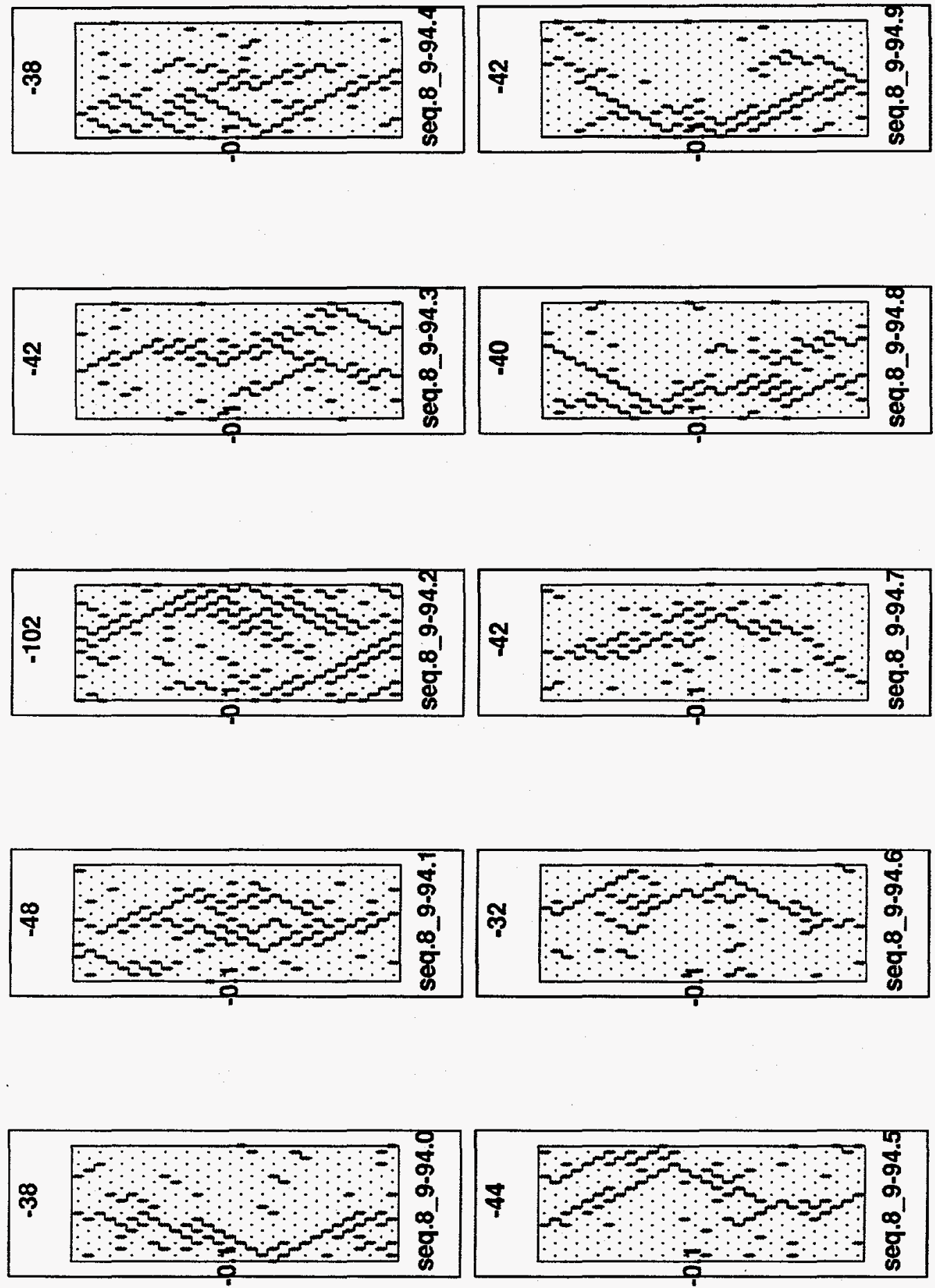
the percolation thresholds for the homogeneous and heterogeneous trials discussed above are in the range 0.22 to 0.34 , and the bimodal trials had $25 \%$ of the sites with strength of $2 \mathrm{MPa}$, but approximately half of these sites have positive values for $m_{1}$, indicating that only one-eighth of the sites were easy to break, and that the weak sites had little effect on the behavior.

Investigation of this effect is beyond the scope of this thesis and will be left for future work. In summary, the inclusion of $25 \%$ very weak sites did not dramatically change the macroscopic behavior.

\subsubsection{Effect of Mean Site Strength}

The dependence of mean peak strength on mean site strength was also evaluated for several different mean site strengths using a $10 \times 30$ triangular lattice, with $p_{c}=0.1 \mathrm{MPa}$ and $m_{1}$ in \pm 0.5 and \pm 0.7 . Table 5.3-1 summarizes the sets of trials conducted to evaluate the effect of changing mean site strength and presents the results in terms of mean peak strength and mean percolation threshold. Four different values of mean site strength and two ranges of $m_{1}$ were used. Data for several of the sets with mean strength of $20 \mathrm{MPa}$ have been discussed in earlier sections. Summary data for the trials at other mean site strengths are presented in the Appendix. Results presented in Table 5.3-1 show that changing the mean site strength had no significant effect on the percolation threshold.

Figure 5.3-20 shows mean ultimate strength vs mean site strength for sets of trials with $m_{1}$ in \pm 0.5 and \pm 0.7 . This figure shows that for both ranges of $m_{1}$ there is a linear dependence of mean ultimate strength on mean site strength. Moreover, this figure shows that the increase in ultimate strength with mean site strength is lowered by increasing the range of $m_{1}$, which indicates that increasing the local stress heterogeneity has a noticeable effect on the relation of ultimate strength and site strength. For the higher ranges of $m_{1}$ more energy is available for cracking of sites so crack interaction may become important at lower levels of stress. This result is preliminary and is for uniform distributions of $m_{1}$ and $T_{g}$ only. More work needs to be done to assess this effect. Possible avenues for investigation of this result are presented in Chapter 7, 
Table 5.3-1. Dependence of ultimate strength on mean site strength for uniaxial simulations using $10 \times 30$ arrays.

\begin{tabular}{lccccc}
\hline $\begin{array}{c}\text { Site strength } \\
\text { distribution }\end{array}$ & $\begin{array}{c}\text { Mean site } \\
\text { strength }(\mathrm{MPa})\end{array}$ & $m_{1}$ & $\begin{array}{c}\Delta \sigma_{1} \\
(\mathrm{MPa})\end{array}$ & $\begin{array}{c}\sigma_{\mathrm{pk}} \\
(\mathrm{MPa})\end{array}$ & $p_{c}$ \\
\hline $20 \pm .2$ & 20 & 0.5 & 2 & $42.4 \pm 2.5$ & $0.18 \pm 0.03$ \\
$20 \pm 19.9$ & 20 & 0.5 & 2 & $41.4 \pm 2.8$ & $0.29 \pm 0.03$ \\
bimodal & 20 & 0.5 & 2 & $48.6 \pm 17.6$ & $0.31 \pm 0.07$ \\
$26 \pm .26$ & 26 & 0.5 & 10 & $55.0 \pm 3.40$ & $0.22 \pm 0.05$ \\
$35 \pm 34$ & 35 & 0.5 & 2 & $67.8 \pm 8.5$ & $0.31 \pm 0.04$ \\
$50 \pm 49.5$ & 50 & 0.5 & 2 & $109.2 \pm 20.70$ & $0.33 \pm 0.09$ \\
& & & & & \\
$20 \pm 19.9$ & 20 & 0.7 & 1 & $32.3 \pm 6.20$ & $0.34 \pm 0.06$ \\
$35 \pm 34$ & 35 & 0.7 & 2 & $50.4 \pm 7.0$ & $0.32 \pm 0.05$ \\
$50 \pm 49.5$ & 50 & 0.7 & 2 & $76.0 \pm 12.6$ & $0.33 \pm 0.05$ \\
\hline
\end{tabular}

which describes future work. It is also important to note that the zero intercept for the linear fit is approximately zero. This indicates that the model is providing sensible results.

It is important to note that when heterogeneous site strength distributions are used the peak strength for any given trial may be considerably different from the mean, as the error bars show. Two important results are shown in this figure. First, for trials with mean site strength of $20 \mathrm{MPa}$, neither increasing the width of the site strength distribution, nor changing the type of distribution from uniform to bimodal, had a significant effect on the mean ultimate strength. This indicates that peak strength may be more dependent on mean site strength than on the distribution. Second, mean ultimate strength increases linearly with mean site strength, indicating that the field-theory model is behaving in a predictable manner. 


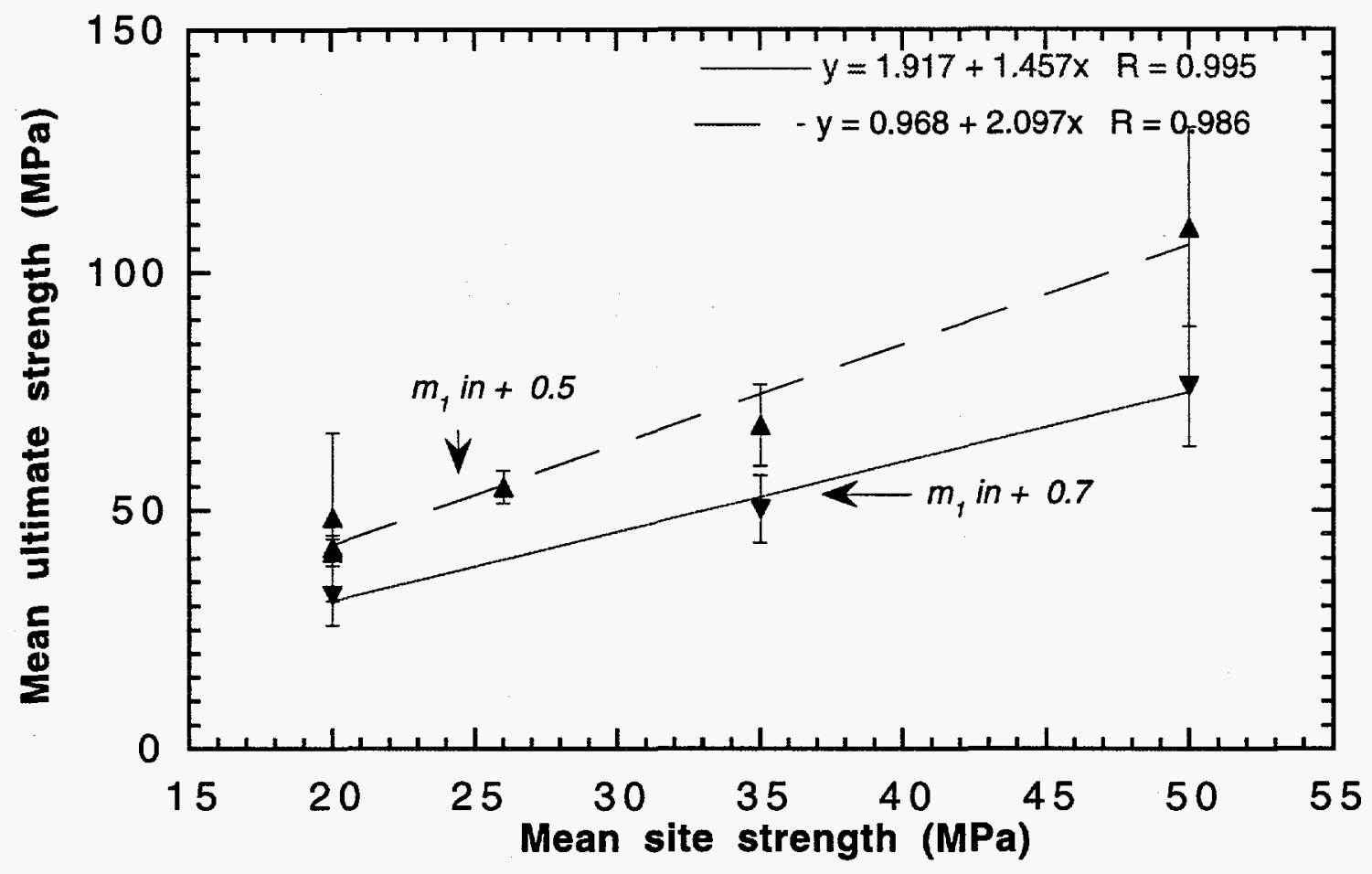

Figure 5.3-20. Dependence of mean ultimate strength on mean site strength for trials computed using $m_{1}$ in two different ranges. 


\subsubsection{Summary and Discussion of Site Strength Study on Triangular Lattice}

The analysis of the effect of site strength distribution provides the following results.

(1) Increasing the heterogeneity in the site strength distribution does not affect mean peak strength, but does cause increased variability in the macroscopic stress-strain behavior, and an increase in the number of sites cracked at fracture (i.e., an increase in percolation threshold).

(2) The sequence of cracking is much different for homogeneous and heterogeneous site strength distributions. For homogeneous site strength, no site cracking is observed as applied stress is increased until an initial site is cracked, and this leads to formation of a cluster that spans the lattice. Heterogeneous site strength distribution causes a population of randomly distributed cracks to form at low stress levels.

(3) Arrays with heterogeneous site strength show more reduction in deformation modulus, and the normalized crack strain energy at failure is lower for heterogeneous site strength distributions than for homogeneous site strength distributions. This means that the contribution of crack strain energy for the heterogeneous site strengths is a smaller percentage of the total strain energy. Thus, the damage caused by the randomly located cracks at low stress has an effect, requiring less energy to fracture the material. At failure, the contribution of crack strain energy in heterogeneous site strength distributions is a lower percentage of the total than in homogeneous site strength distributions. This relates to crack interaction.

(4) Similar mean peak strengths are predicted for both homogeneous and heterogeneous uniform site strength distributions. This suggests that the mean site strength controls the mean peak strength. This is consistent with the analysis of Ambegaokar et al. (1971) for continuum percolation in broad distributions. These authors show that for a broad distribution the peak in a parameter can be related to the value that the parameter has when the percolation threshold is reached. Moreover, the model shows a linear dependence of mean peak strength on the mean value of site strength. 
(5) Bimodal site strength distributions show stress-strain behavior that is similar to that for the homogeneous site strength distributions, but with a wider range of mean strengths. This has implications for material design, as it aids in characterizing the behavior of types of composite materials. However, it is important to note that the effect of the bimodal site strength distribution is not well understood and future work is needed to determine what percentage of weak or strong sites must be included to decrease/increase the mean peak strength. This may also be explained by the analysis of Ambegaokar et al. (1971) mentioned above.

Even though the model indicates that the mean peak strength depends more strongly on mean site strength than on site strength distribution, it is important to note that except for one case, only uniform strength distributions were used. It is apparent from the results that more work needs to be done to understand how bimodal or multimodal distributions of site strength affect macroscopic behavior, and a study could be conducted where incrementally altered bimodal distributions are used to determine the proportion of weak/strong sites needed to affect the macroscopic properties. Weibull and power-law distributions also need to be examined.

\subsection{Effect of Lattice Geometry-Perturbed Triangular Lattice}

The purpose of this section is to show how perturbation of the site locations of triangular lattices affects the behavior predicted by the field-theory model. In particular, one of the purposes of this section is to evaluate whether the perturbation of site location would introduce stepping of cracked sites and produce en-echelon crack patterns. The algorithms for perturbing the location of sites on triangular lattices are discussed in Section 4.3, along with discussion of the techniques used to determine crack length and connecting cracks for the perturbed lattice. The perturbed lattices all used the same number of sites in essentially the same area as the unperturbed lattices, so that the macroscopic site density is constant over the array, but is locally heterogeneous. 
To study the effect of lattice site perturbation, simulations were conducted as follows. First, a trial was computed on a regular triangular lattice; this lattice was then perturbed by allowing each point to be moved to a location within a maximum perturbation radius, $r_{\mathrm{p}_{\max }}$ of the original point, and the amount of perturbation introduced into the array can be characterized using this radius. For a regular lattice the maximum perturbation radius is zero. To study how the amount of perturbation affects behavior predicted by the model it is useful to compare lattices produced with $r$ specified in different ranges. For this study the lattices were perturbed using maximum perturbation radii of $0.0,0.04 l_{c}$, and $0.4 l_{c}$, where $l_{c}$ is the lattice constant. Trials were computed using the perturbed lattice sites but the same input files of site strength $T_{g}$ and geometric parameter $m_{1}$. In this manner the effect of increasing the amount of heterogeneity in site location was determined.

The effect of lattice site perturbation was examined for conditions of homogeneous and heterogeneous site strength with $m_{1}$ in the range \pm 0.7 for unconfined simulations on $10 \times 30$ arrays. Following the structure used in the previous sections of this thesis, the case of homogeneous site strength is discussed first.

\subsubsection{Homogeneous Site Strength Distribution}

In this section single trials are presented that have been computed with perturbation radii in the ranges $r=0.00 l_{c}, 0 \leq r \leq 0.04 l_{c^{\prime}}$ and $0 \leq r \leq 0.40 l_{c}$. These will be denoted as trials r.00, r.04 and r.40, respectively, in the following discussion.

\section{Stress-Strain}

Stress-strain curves for three trials are shown in Figure 5.4-1. These curves are very similar to each other and show behavior similar to the stress-strain curves for other trials computed for homogeneous site strength distributions. Stress-strain for the unperturbed trial (r.00) shows some strain-softening at an earlier stage than observed for the two perturbed trials, and the percolating cluster is formed at a lower value of strain for the unperturbed trial. Thus, in this 
set of trials, perturbing the lattice increased the strain at which strain-softening and percolation occurred.

\section{Applied Stress vs Normalized Crack Strain Energy}

The plot of applied stress vs normalized crack strain energy is shown in Figure 5.4-2. This figure shows similar behavior for all three trials and illustrates that all sites cracked at the same applied stress. However, the unperturbed trial shows the least amount of normalized crack strain energy.

\section{Number of Cracks vs Crack Strain Energy}

The number of cracks is plotted as a function of crack strain energy in Figure 5.4-3. This figure shows a large increase in crack strain energy as the number of cracks increase, as is typical of homogeneous site strength simulations. The trend of each data set is nearly linear with a distinct upward trend at the highest number of cracks. The curves for each of the three trials are parallel up to a normalized crack strain energy of 0.3 ; then the curve for the unperturbed trial shows an accumulation of cracks with minimal gain in crack strain energy.

\section{Crack Patterns}

The effect of lattice perturbation on the development of crack pattern is shown in Figures 5.4-4 a-1. These figures show the locations of cracked sites for each of the trials when 4,15 , and 30 sites are cracked, and at the percolation threshold. Figures 5.4-4 a-c show that the effect of lattice perturbation is immediately evident after only four sites have broken. The crack pattern for the unperturbed trial (r.00, Figure 5.4-4 a) shows two clusters, marked A and B. A is a cluster of three sites in the lower central region of the figure, and B is a singular cluster slightly below A. Figure 5.4-4 b shows the crack pattern after four sites have broken for trial r.04. These crack patterns show one cluster (A) of four sites in the lower central region, and three of the sites are coincident with those broken in cluster A of r.00. The crack patterns after four sites have broken in r.40 are shown in Figure 5.4-4 c. This figure shows the formation of 
three clusters: A, B, and C. A is a cluster of one that is coincident with a site broken in r.00 and r.04. Clusters $B$ and $C$ are not coincident with sites broken in r.00 or r.04.

Figures 5.4-4 d-f show cluster structure for r.00, r.04, and r.40 after 15 sites have cracked. For r.00 (Figure 5.4-4 d) only one cluster is shown. This cluster, A, includes both clusters A and B of Figure 5.4-4 a and shows that the two clusters A and B have linked and extended to the upper left of the old A and downward beyond the old B, but not as far as the bottom of the array. Cracked sites for trial r.04 (Figure 5.4-4 e) also form one cluster, A, which contains some sites that are common to $\mathrm{A}$ in $\mathrm{r} .00$, but this cluster trends to the upper right and has also extended to the bottom of the array. The crack pattern for trial r.40 shown in Figure 5.4-4 $\mathrm{f}$ is similar to that for trial r.04 as cluster A has grown in much the same way as for r.04, trending to the upper left, and extending to the bottom of the array. Clusters B and C in Figure 5.4-4 f are the same as those as in Figure 5.4-4 c, but a new single cluster $D$ has formed near the bottom of the array.

Crack patterns for the three trials after 30 sites have cracked are shown in Figures $5.4-4 \mathrm{~g}-$ i. The pattern for trial r.00 (Figure 5.4-4 g) shows a single cluster A that has continued to extend in the vertical direction parallel to the maximum principal stress. Figure 5.4-4 h shows that broken sites for r.04 also form one cluster, A, that extends to the upper right with three small branches extending to the upper left. The maximum length of these branches is three sites. The pattern of cracked sites for r.40 shown in Figure 5.4-4 i shows the existence of four clusters, with one cluster, A, dominating. Cluster r.40-A is similar to r.04-A, while clusters r.40-B, C, and D all remain unchanged. Cluster r.40-A also shows branching similar to that shown for r.04-A, with one branch four sites in length.

Crack patterns at the percolation threshold for these trials are shown in Figures 5.4-4 $j-1$. These figures show that the pattern formed for the unperturbed trial r.00 (Figure 5.4-4 $\mathrm{j}$ ) is nearly vertical and bisects the array just to the left of the center line. The patterns for the perturbed trials are quite different and show en-echelon patterns of cracks that step across the array from the lower left to the upper right. The trials r.00 and r.04 show no cracks outside of the percolating cluster, while the trial r.40 shows five cracks that are outside of the critical 


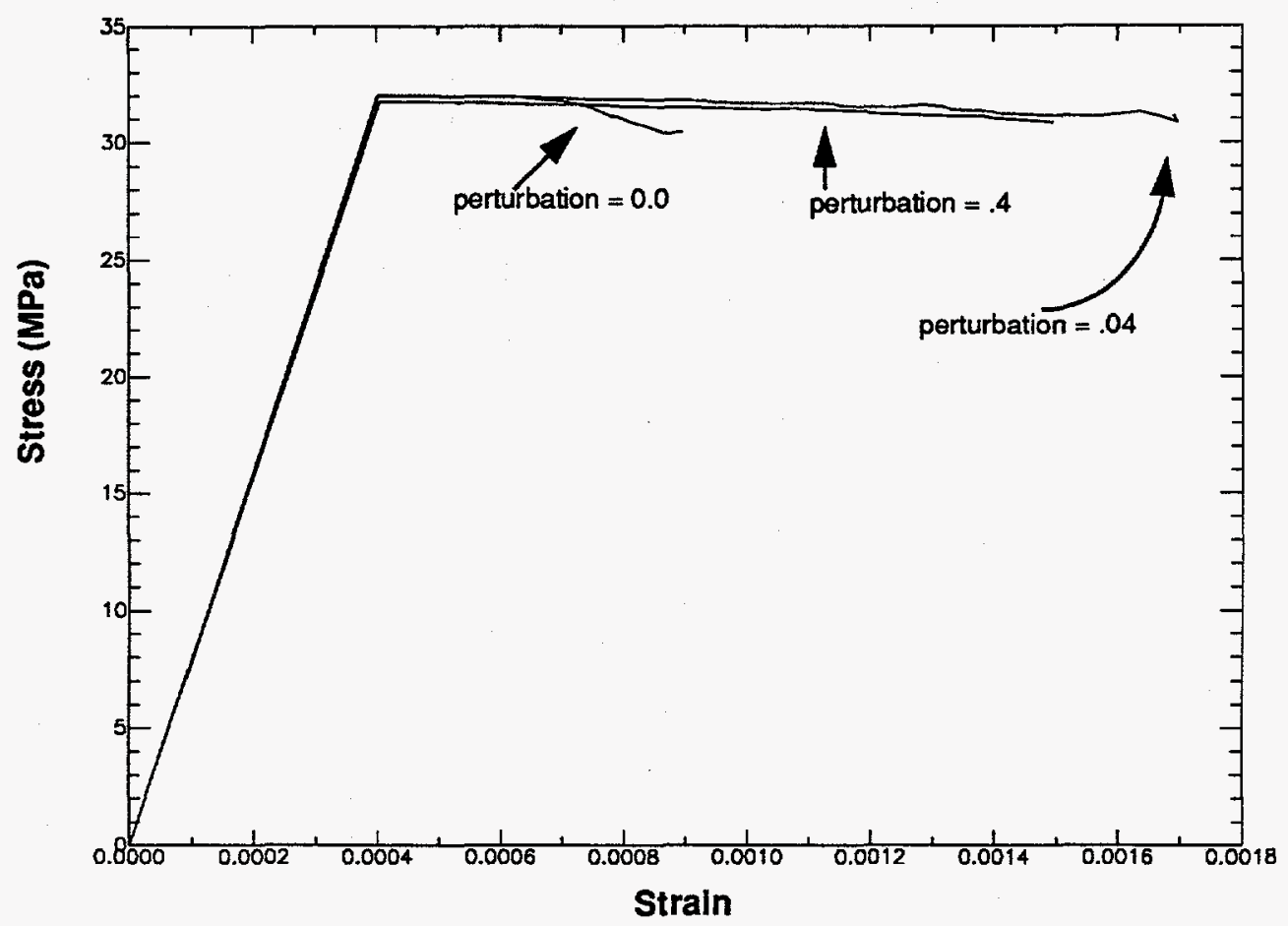

Figure 5.4-1 Effect of lattice perturbation on stress-strain curves for triangular lattice with homogeneous site strength distribution.

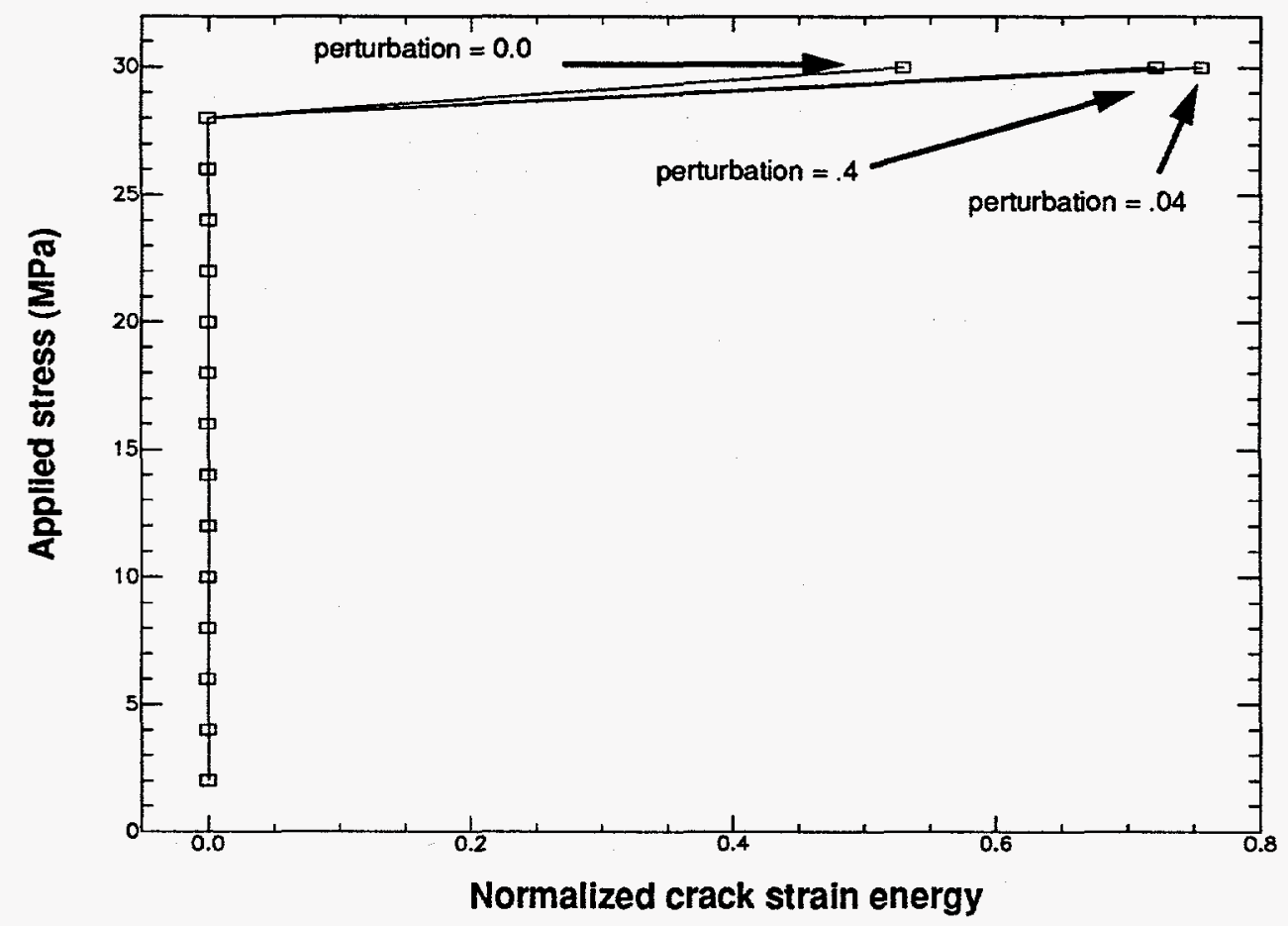

Figure 5.4 - 2 Effect of lattice perturbation on relation of applied stress to normalized crack strain energy for triangular lattice with homogeneous site strength distribution. 


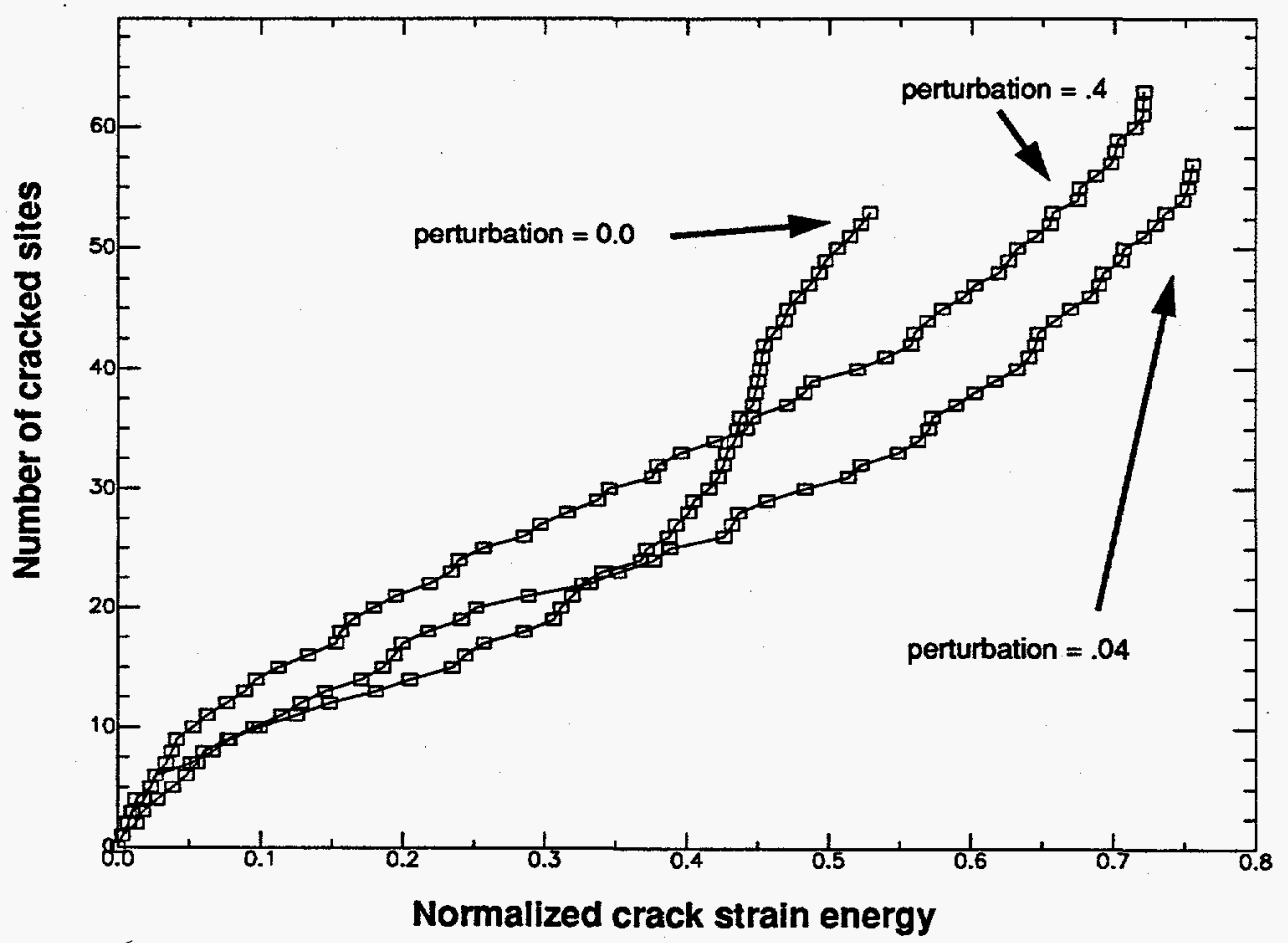

Figure 5.4-3 Effect of lattice perturbation on relation of number of cracked sites to normalized crack strain energy for triangular lattice with homogeneous site strength distribution. 


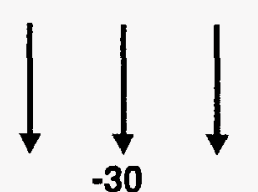

宾
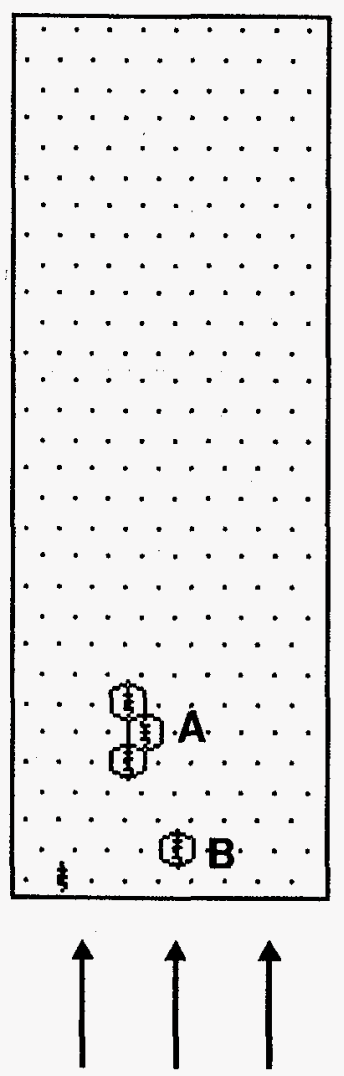

a) $r_{\text {pmax }}=0.0$ $\downarrow \downarrow$
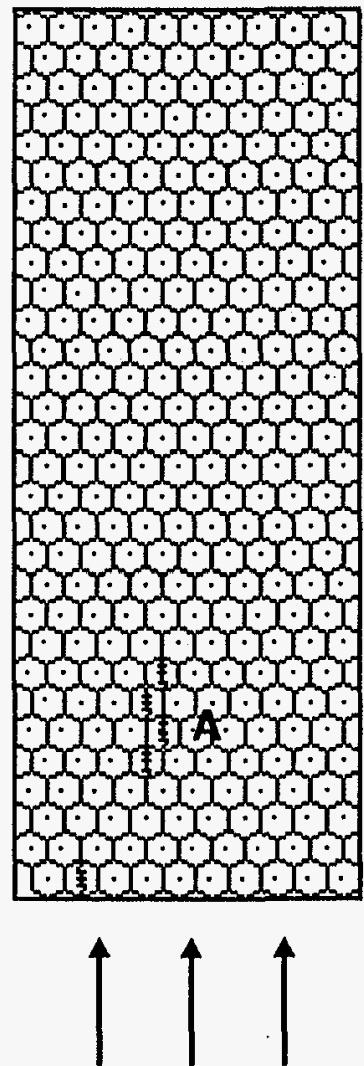

b) $r_{p \max }=0.04$
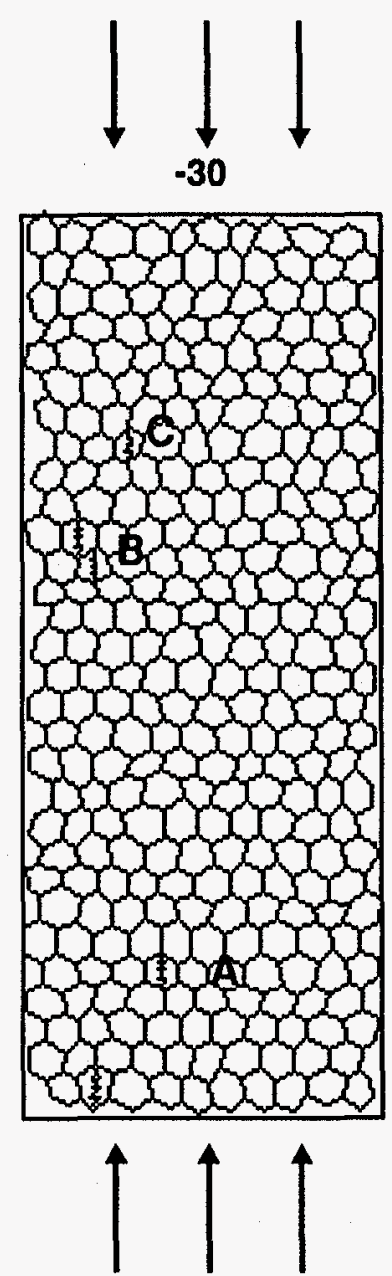

c) $r_{\text {max }}=0.40$

Figure 5.4 - 4. Effect of lattice perturbation on crack pattern development for triangular lattice with homogeneous site strength distribution. Crack patterns shown for one regular and two perturbed trials, when 4,15 , and 30 sites are cracked, and at the percolation threshold. See text for detailed discussion. $a, b, c)$ crack patterns when 4 sites are cracked. 


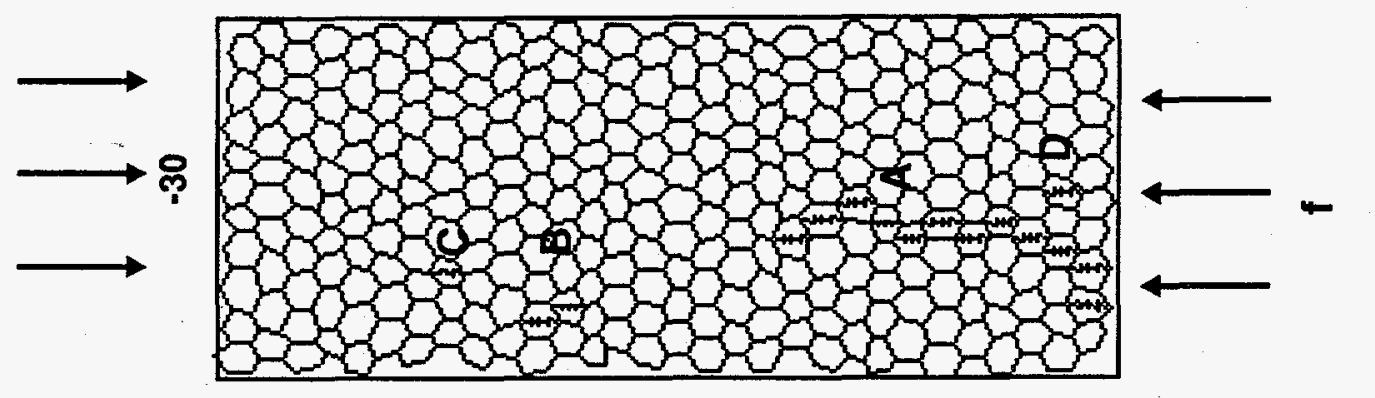

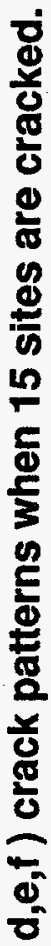
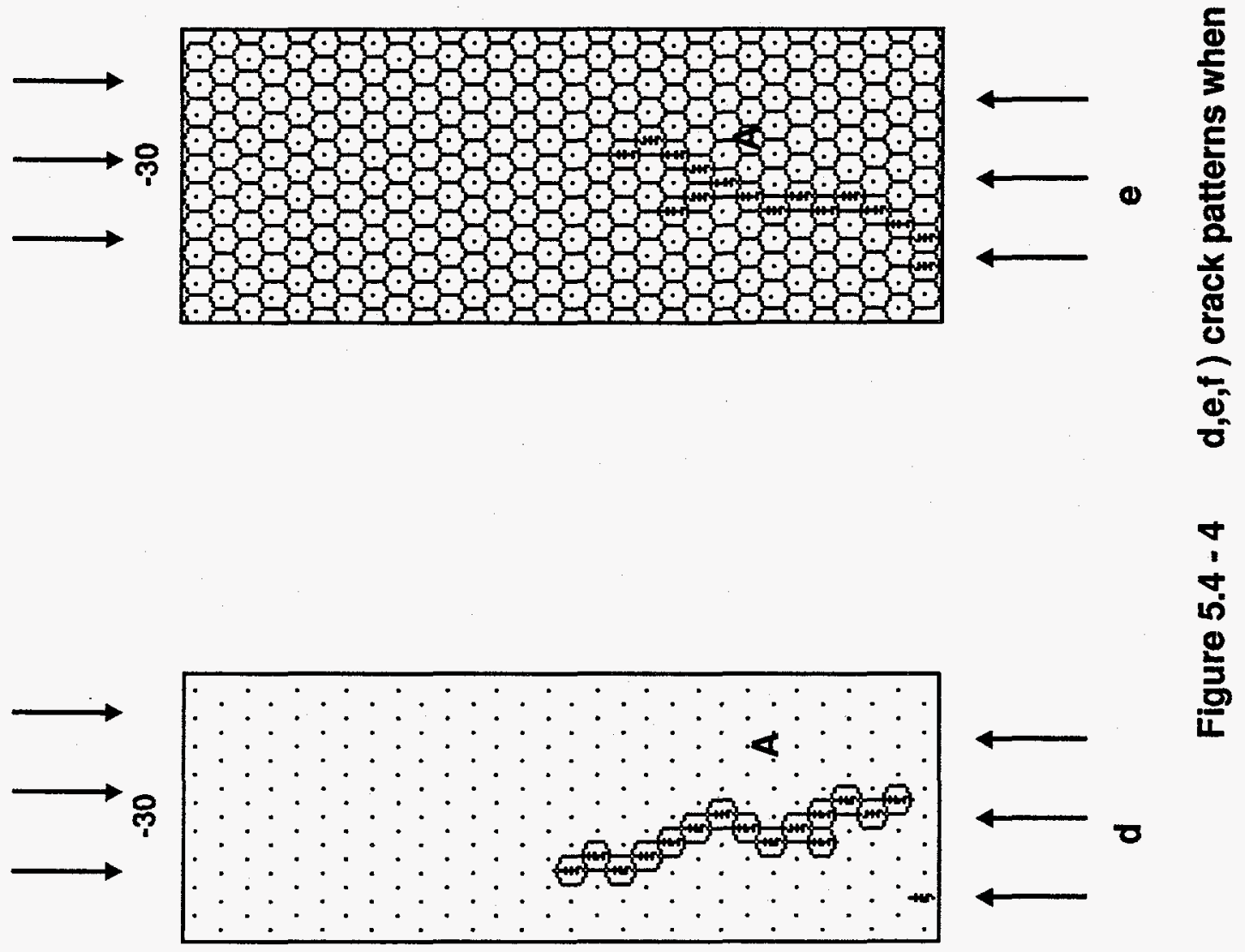

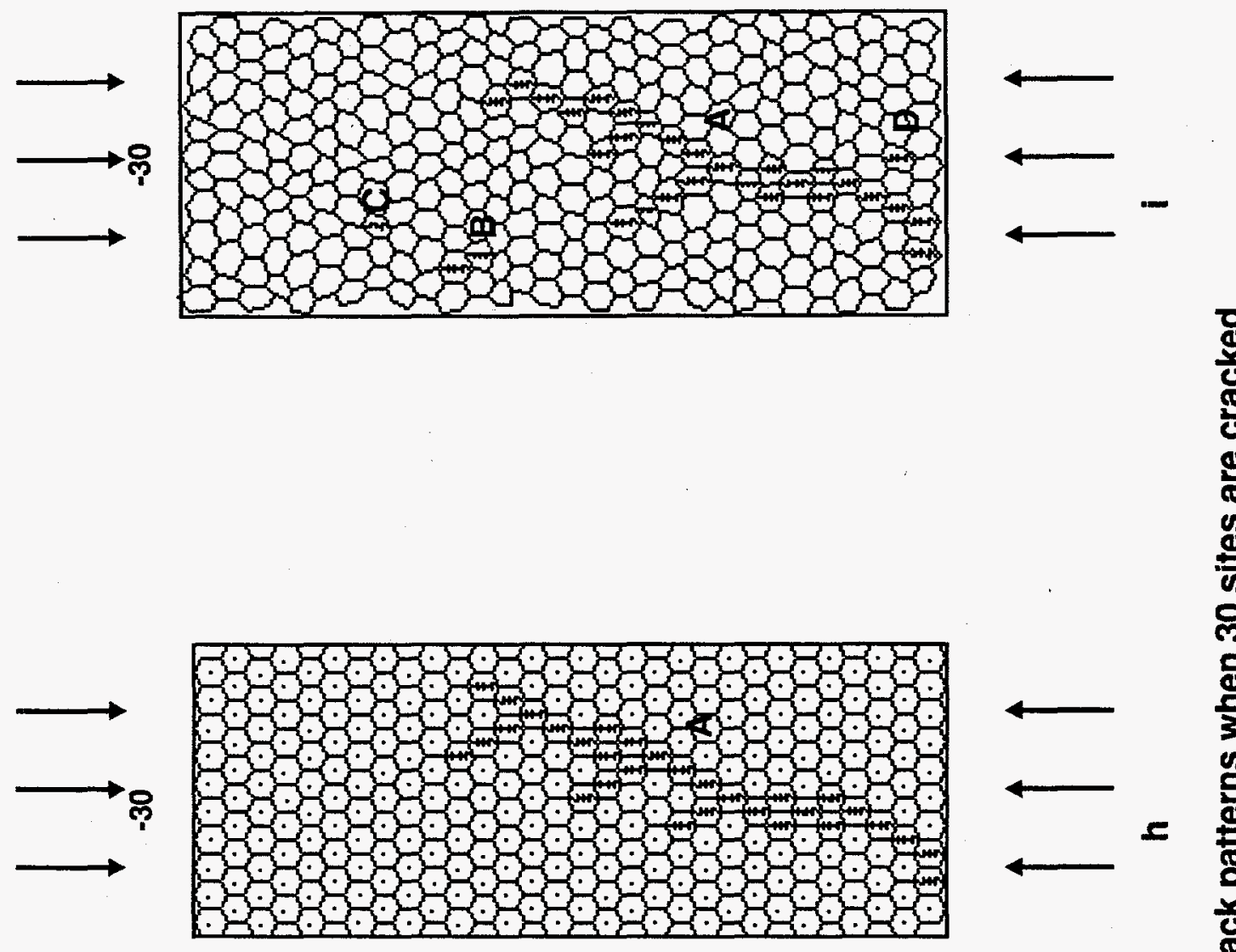

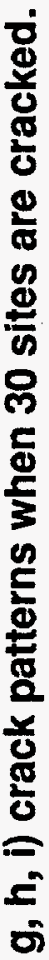

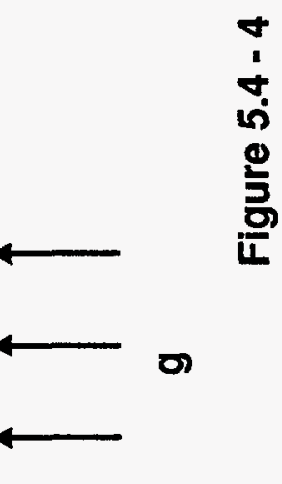



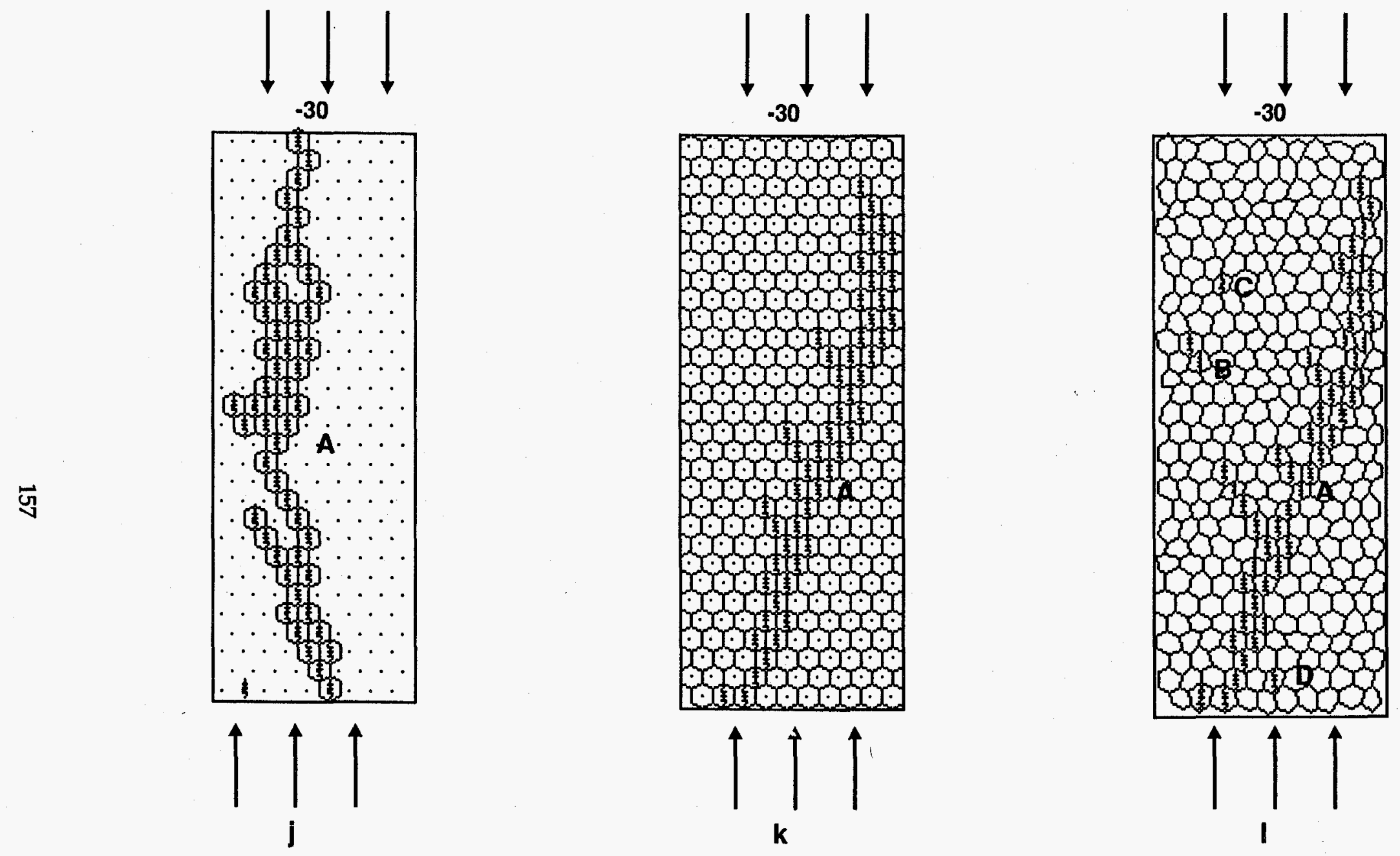

Figure 5.4 - $4 \quad j, k, l)$ crack patterns at or near the percolation threshold. 
cluster. The pattern for r. 00 shows one branching crack while r.04 shows two branching cracks with length of 2 sites. The pattern for r.40 shows three branching cracks, one of which has a length of four sites. This may imply that perturbation of the lattice site location has increased the percolation threshold and changed the scale of localization of the site cracking.

\section{Discussion}

Results show that for these trials, stress-strain behavior for perturbed and unperturbed trials was similar, as were crack patterns. However, while overall patterns were similar, the crack patterns for the perturbed trials showed crack stepping, and differences in crack patterns for perturbed and unperturbed trials were observed immediately (after four cracks). Increasing heterogeneity in the lattice site locations decreased crack localization and decreased normalized crack strain energy. This is consistent with the postulate that systems with increasing disorder require more energy to break. During the initial phase of cracking (less than $10 \%$ of the sites cracked), the perturbed lattices displayed progressively less strain energy at a given number of cracks. Moreover, for the perturbed lattices, at the percolation threshold a larger proportion of the total strain energy was due to cracks. Data for percolation threshold and peak strength for these trials are discussed in Section 5.4.3.

\subsubsection{Heterogeneous Site Strength Distribution}

In this section results are presented for a set of three trials computed with heterogeneous site strength distribution and lattice perturbations identical to those described in the previous section.

\section{Stress-Strain}

Stress-strain curves for three trials r.00, r.04, and r.40 are shown in Figure 5.4-5. These curves show that all trials follow the same behavior up to an axial stress of approximately 26 MPa. At this stress level the unperturbed trial (r.00) shows strain-softening that leads to failure, while trial r.04 shows strain-hardening to approximately $32 \mathrm{MPa}$ and then strainsoftening to failure. Trial r.40 is similar to r.04, but shows a further step of strain-hardening to 
$33 \mathrm{MPa}$ before strain-softening to failure. Thus, for these trials, increasing the range of the perturbation radius caused an increase in peak strength.

\section{Applied Stress vs Normalized Crack Strain Energy}

Plots showing applied stress vs normalized crack strain energy for these trials are shown in Figure 5.4-6. The data for each of the trials are very similar and show that normalized crack strain energy increases slowly and linearly with applied stress up to approximately $20 \mathrm{MPa}$, and then increases sharply as cracks are formed. This plot shows the same trend as observed in the stress-strain curve, i.e., that behavior for the three trials is quite similar up to $24 \mathrm{MPa}$. Also, increasing the range of the perturbation radius increases the level of applied stress

- required to produce a given level of normalized crack strain energy. This implies that for the perturbed lattice, more energy is required to produce the same amount of damage. This is consistent with the results for the homogeneous site strength distribution.

\section{Number of Cracks vs Normalized Crack Strain Energy}

The number of cracks vs normalized crack strain energy for these trials is plotted in Figure 5.4-7. This plot shows similar behavior for the three trials up to a normalized crack strain energy value of about 0.3 . After this point the data show that for the perturbed lattices, more cracks are required to produce the same levels of normalized crack strain energy. On this figure the normalized crack strain energy at 25,45 , and 65 cracks is marked. These values show that the normalized crack strain energy values are similar for 25 and 45 broken sites. At 65 sites broken, the normalized crack strain energy value for $\mathrm{r} .00$ is much different from that for $\mathrm{r} .04$ and r.40. This is consistent with the observed crack patterns discussed in the next section.

\section{Crack Patterns}

The crack patterns for these trials are discussed by showing the locations of cracked sites when 25,45 , and 65 sites were cracked and when the percolation threshold was reached (see Figures 5.4-8 a-1). Locations of cracked sites for the first 25 cracks in each of the three trials are shown in Figure 5.4-8 a, b, and c. As expected, the cracks are widely distributed in contrast to 


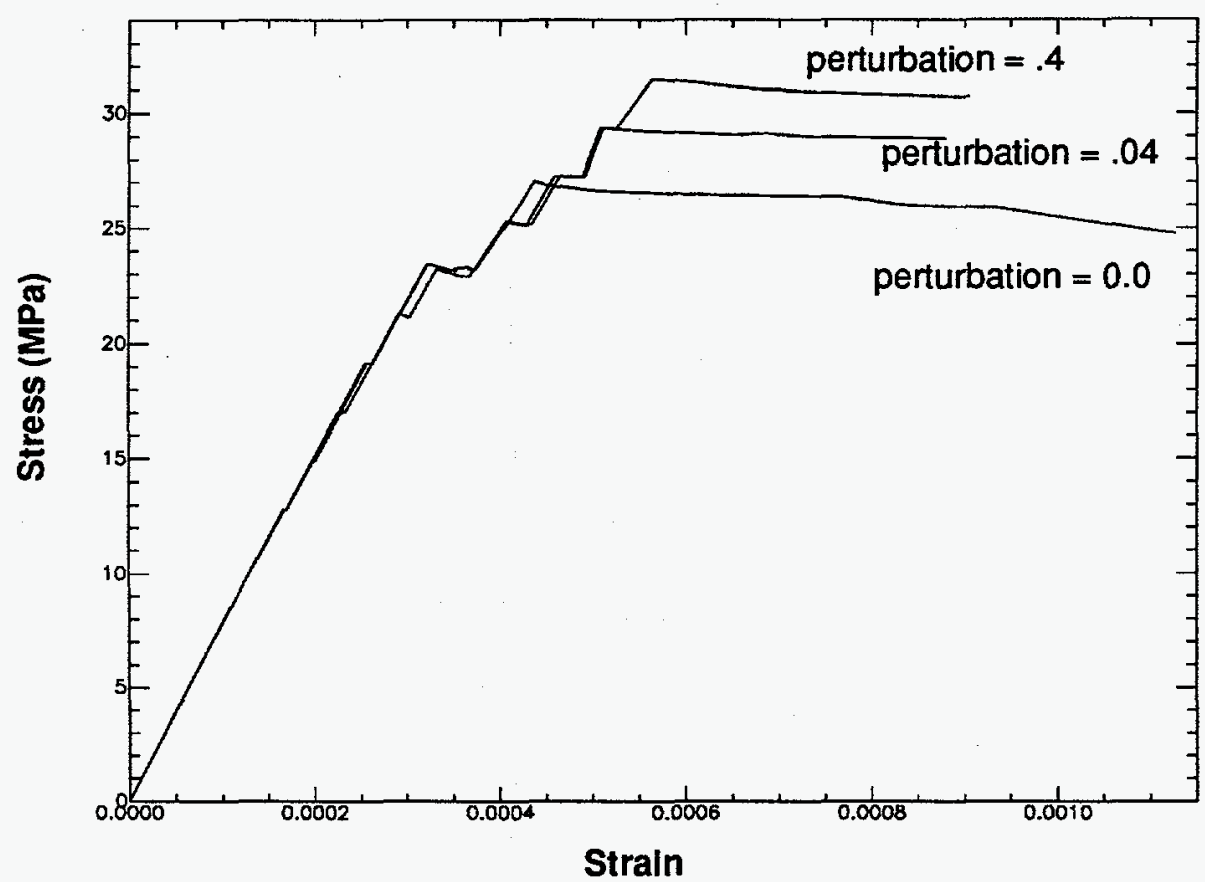

Figure 5.4-5 Effect of lattice perturbation on stress-strain curves for triangular lattice with heterogeneous site strength distribution.

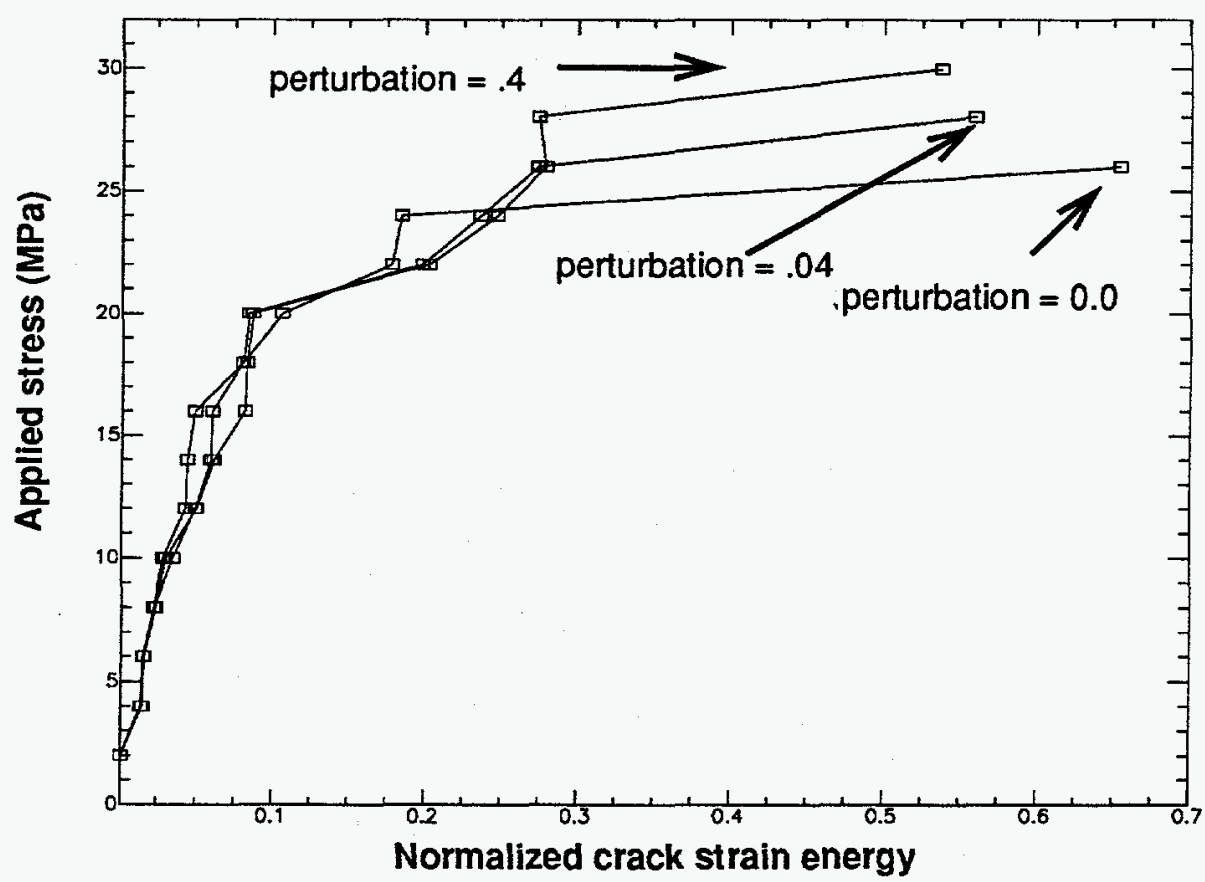

Figure 5.4 - 6 Effect of lattice perturbation on relation of applied stress to normalized crack strain energy for a triangular lattice with heterogeneous site strength distribution. 


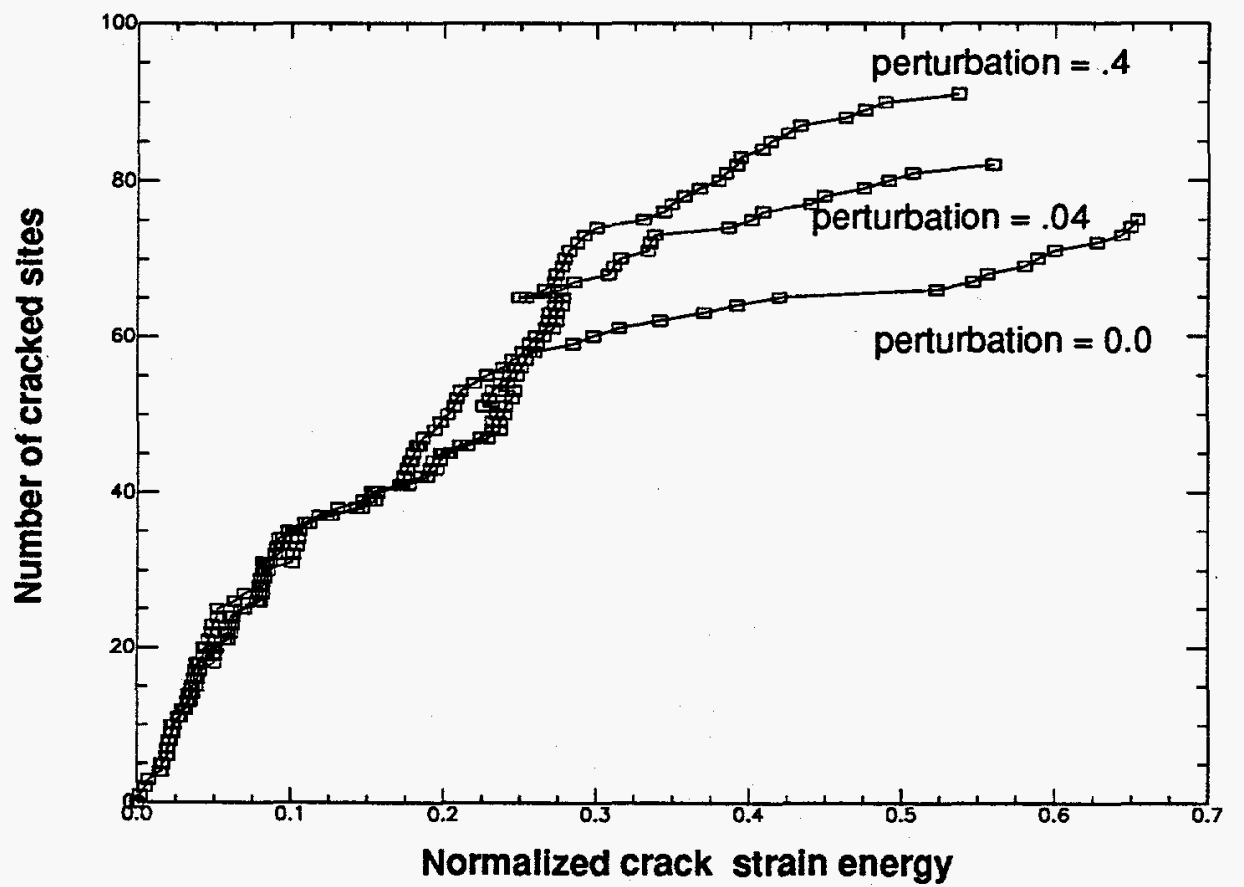

Figure 5.4 - 7 Effect of lattice perturbation on relation of number of cracked sites to normalized crack strain energy for triangular lattice with heterogeneous site strength distribution. 


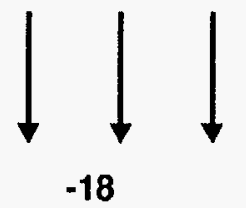

호

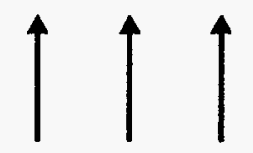

a) $r_{\text {pmax }}=0.0$
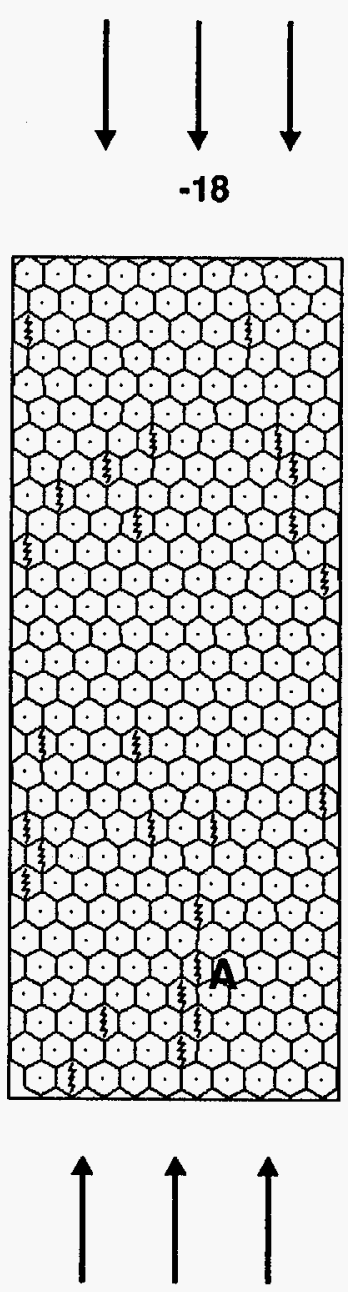

b) $r_{\text {pmax }}=0.04$
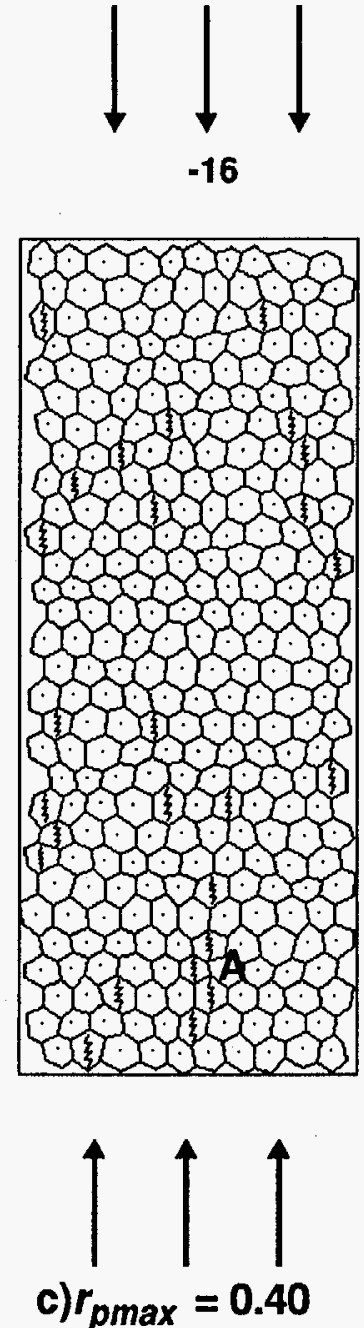

Figure 5.4 - 8. Effect of perturbation of triangular lattice on crack patterns for trials computed using a heterogeneous site strength distribution. a,b,c) crack patterns for 25 cracked sites. 

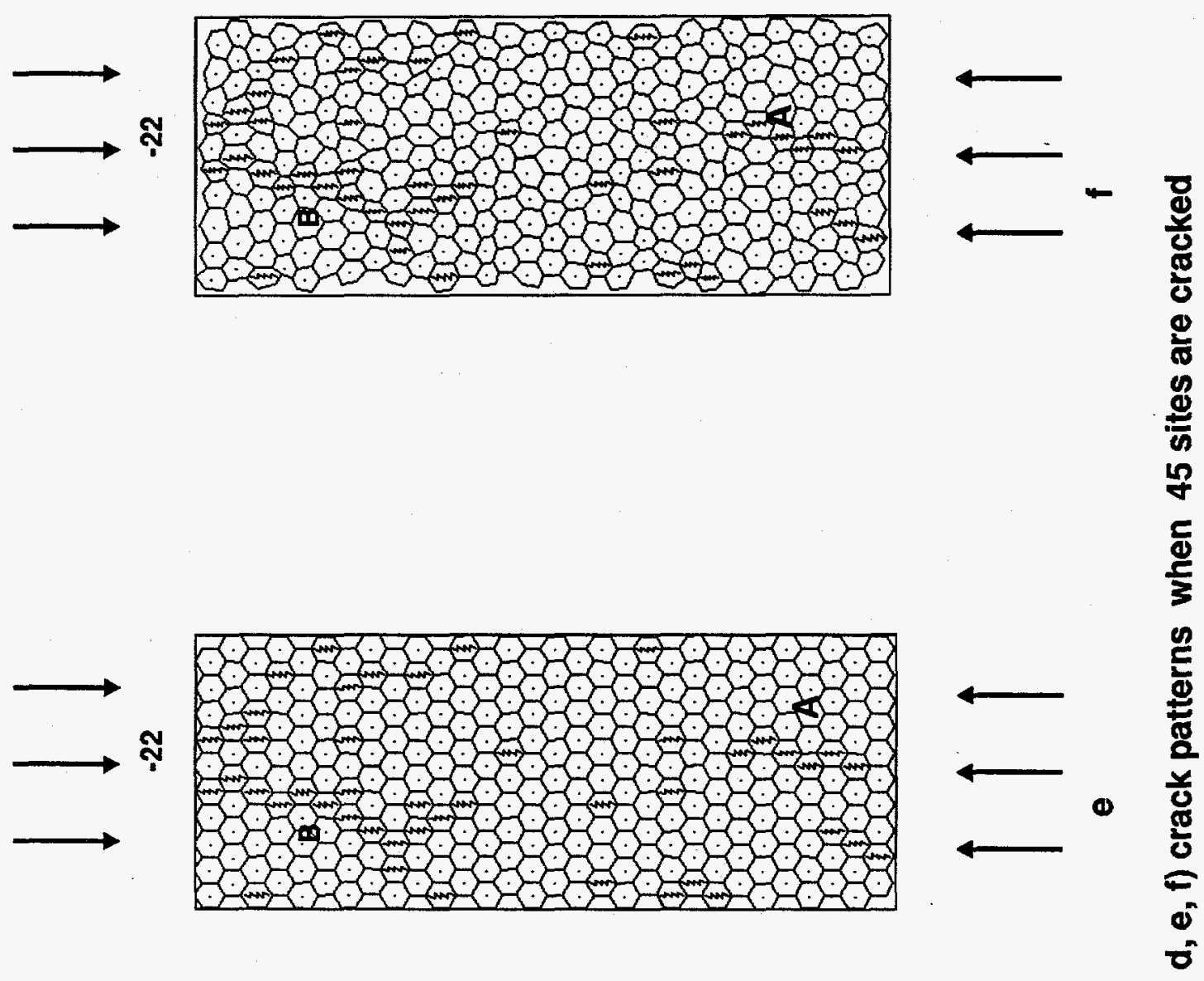

$\infty$
0
0
$\frac{9}{6}$
$\frac{9}{4}$
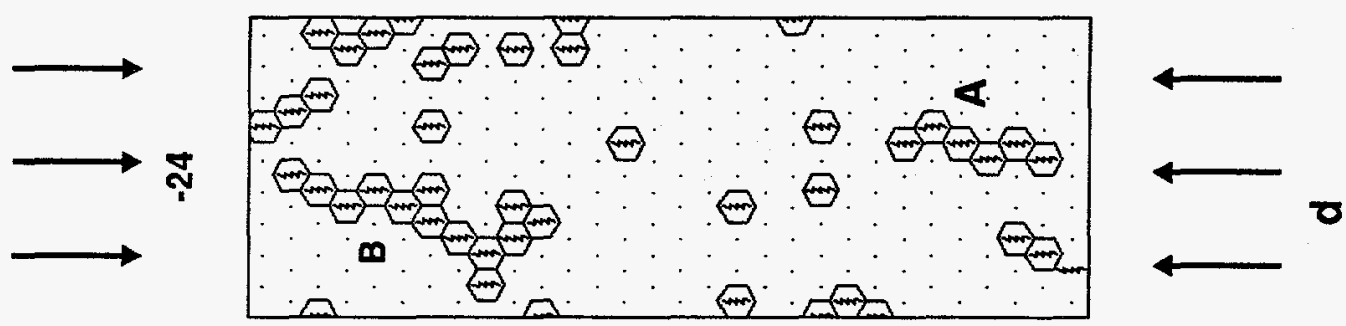

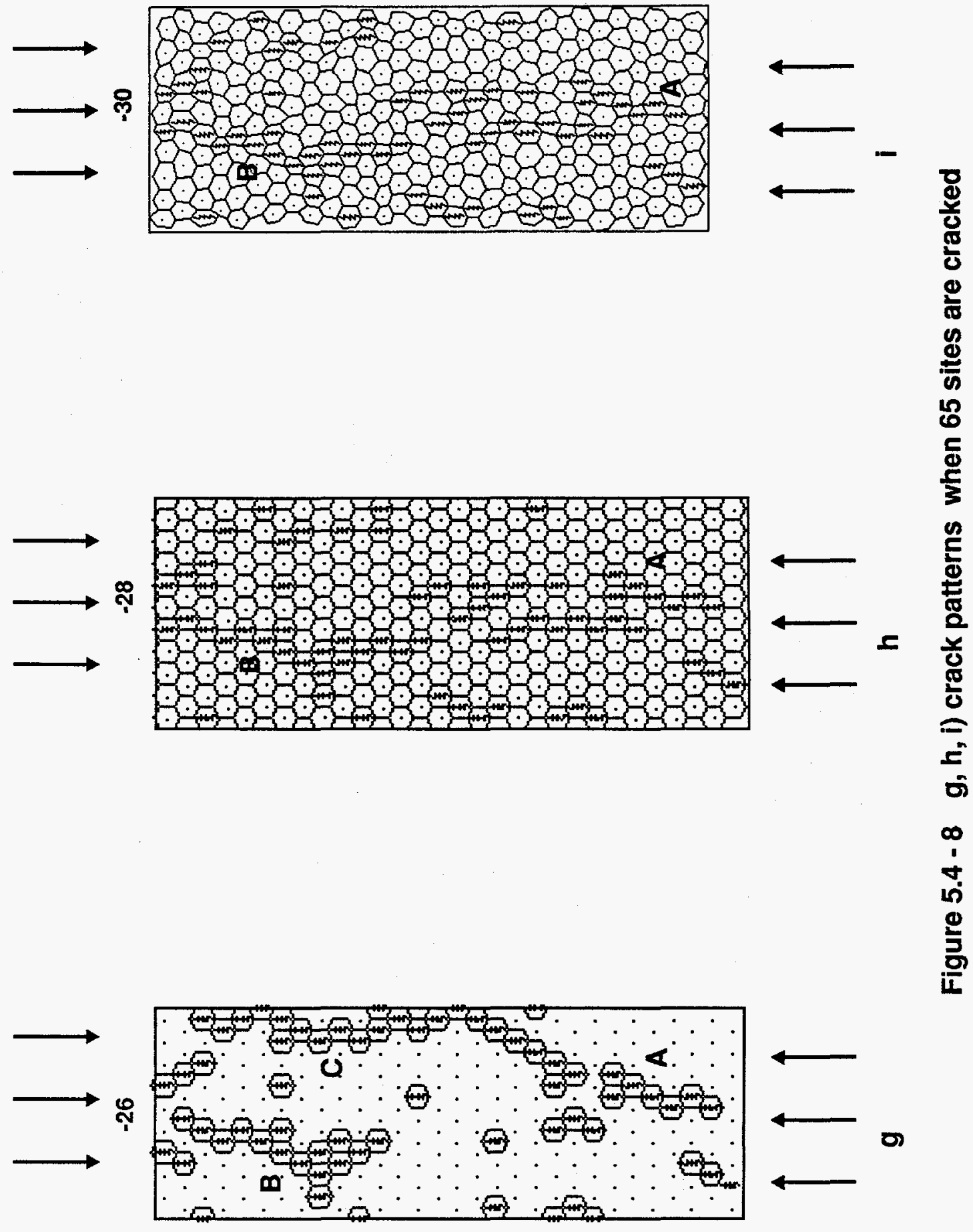

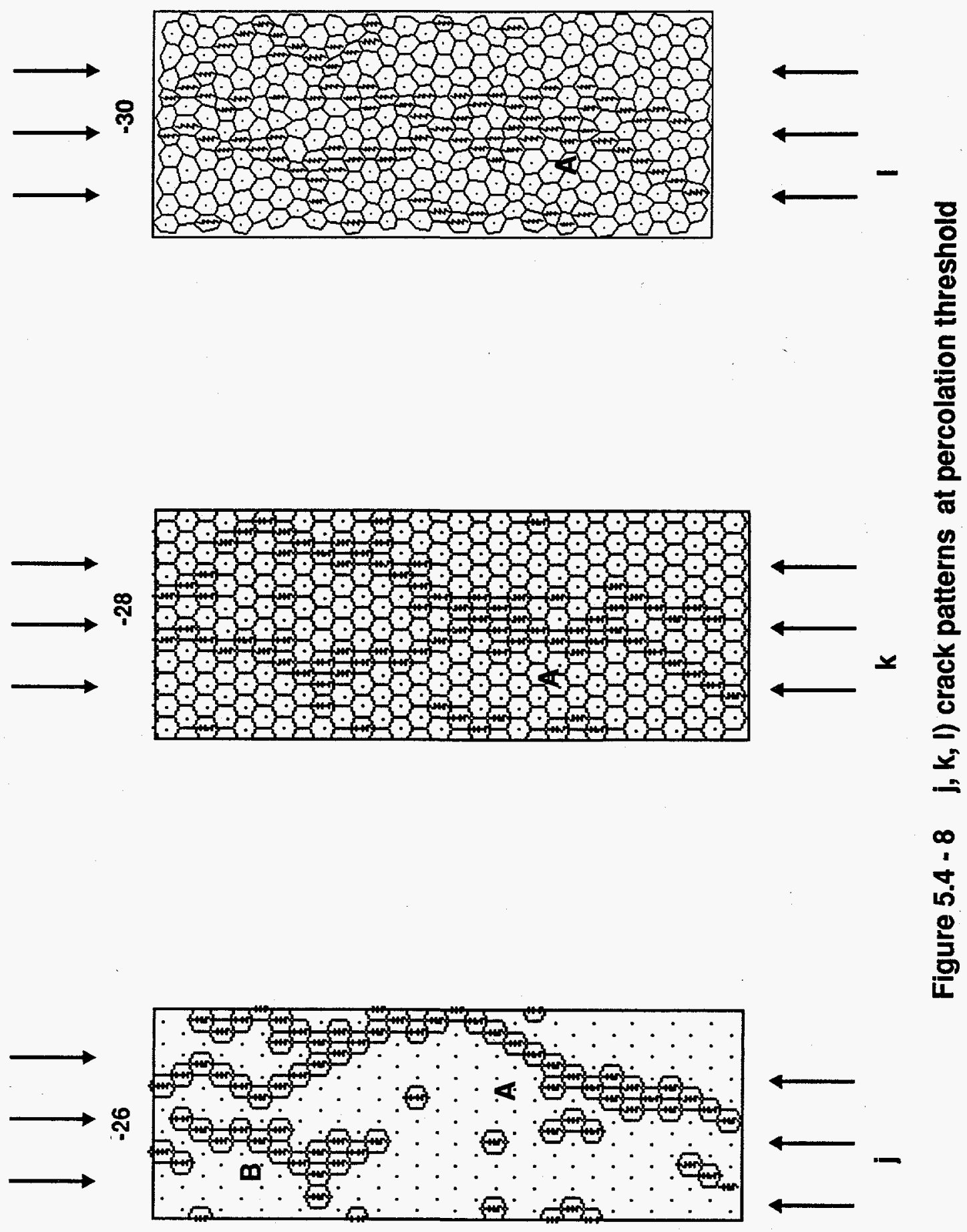
the pattern for the homogeneous site strength case. Patterns are very similar for all three trials and clusters contain one or two sites; each perturbed trial (r.04 and r.40) has a cluster of four sites shown at A. The unperturbed trial shows a cluster of two sites at this location (A). This is in contrast to the homogeneous site strength case where a difference in patterns was noticed immediately.

The crack patterns for these trials when 45 sites are broken are shown in Figures 5.4-8 d, e, and f. Again the patterns are similar for the three trials, with the cluster at A having extended vertically and a larger cluster having formed in the upper left center at B. Thus at $15 \%$ sites broken, no divergence of the pattern is shown.

The pattern of cracks for 65 broken sites is shown in Figures $5.4-8 \mathrm{~g}, \mathrm{~h}$, and $\mathrm{i}$. For the unperturbed trial (r.00, Figure 5.4-8 g) a new and dominant cluster, $\mathrm{C}$, has formed along the right side of the array, extending from the upper right corner to the top of cluster A. Moreover, clusters $A$ and $B$ show very little change from that observed at 45 cracks for this trial. The trials r.04 and r.40 show a much different pattern of cracking in this interval. For these trials, clusters $A$ and $B$ have extended toward each other and no cluster equivalent to $C$ in trial r.00 has formed.

Crack patterns at the percolation threshold for these trials are shown in Figures 5.4-8 $j, k$, and 1. These show more distributed cracks as expected for the heterogeneous site strength distribution.

This brief analysis of Figure 5.4-8 indicates that for this trial, divergence in crack patterns occurs after approximately $15 \%$ of the sites are cracked. Moreover, it is important to notice that the trials with perturbed lattices have similar crack patterns and that these patterns are significantly different from the pattern for the unperturbed lattice. This is consistent with the results from the trials with homogeneous site strength. However, the patterns for the perturbed lattices are more like patterns for the unperturbed trial than was noticed for the homogeneous site strength trials discussed above. The perturbed trials show more en-echelon behavior as they step nearly across the array while the unperturbed trials only step one half of 
the way across. The crack patterns are also consistent with the plots for number of cracks vs normalized crack strain energy. This can be observed as follows. The values of normalized crack strain energy at 25, 45, and 65 cracks for each of these trials are marked on Figure 5.4-7. These values show that the normalized crack strain energy values are similar for all three trials when 25 and 45 sites are broken, as is expected from the crack patterns. However, when 65 sites are broken, the normalized crack strain energy value for $r .00$ is much different from that for r.04 and r.40. This is consistent with the divergence in crack patterns at this number of cracked sites.

\subsubsection{Summary and Discussion for Perturbed Triangular Lattice}

Summary data for the perturbed triangular trials in given in Table 5.4-1. This table presents percolation thresholds and peak stresses for the homogeneous and heterogeneous site strength trials discussed above, and shows that for these trials increasing the range of the perturbation radius caused an increase in the percolation threshold for both the homogeneous and heterogeneous site strength cases. Moreover, the homogeneous site strength trials had lower percolation thresholds as expected. The peak strength values for the homogeneous trials were all very similar, while the increase in lattice perturbation caused an increase in peak strength for the heterogeneous site strength trials. These results are preliminary and reflect only one set of trials; more extensive study is required to verify the findings.

These results may be explained as follows. When a site location is perturbed, it is moved in regard to the stress field and if there is a stress gradient it will be relocated either up gradient, down gradient, or along the gradient. Then, compared to the unperturbed lattice, sites on the perturbed lattice will be more likely to break if they are moved up gradient, and less likely to break if they are moved down gradient. Sites moved along the gradient will have the same conditions as those on the unperturbed lattice. Thus, differences in sequence of cracking between the perturbed and unperturbed lattices will occur only when a perturbed site has been moved up gradient. That is because the sites that are moved into a region of higher stress will have a larger stress difference and thus have a greater stress perturbation $\rho$. These sites will 
Table 5.4-1. Data for single trials for perturbed triangular lattice.

\begin{tabular}{ccccccccccccc}
\hline Date & Lat. $^{\mathrm{a}}$ & JSZ & ISZ & $r_{\text {pmax }}$ & $\sigma_{\mathrm{I}}$ & $\Delta \sigma_{\mathrm{I}}$ & $T_{g}$ & $m_{1}$ & Vb & seq & (MPa) & $p_{\mathcal{c}}$ \\
\hline 29-Jun-94 & ptri & 10 & 30 & 0 & 2 & 2 & $20 \pm 0.2$ & .7 & 35.7 & 1 & 32.0 & 0.18 \\
29-Jun-94 & ptri & 10 & 30 & 0.04 & 2 & 2 & $20 \pm 0.2$ & .7 & 35.7 & 1 & 32.0 & 0.19 \\
29-Jun-94 & ptri & 10 & 30 & 0.40 & 2 & 2 & $20 \pm 0.2$ & .7 & 35.7 & 1 & 31.8 & 0.21 \\
& & & & & & & & & & & & \\
27-Jun-94 & ptri & 10 & 30 & 0 & 2 & 2 & $20 \pm 19.9$ & .7 & 35.7 & 0 & 27.1 & 0.25 \\
27-Jun-94 & ptri & 10 & 30 & 0.04 & 2 & 2 & $20 \pm 19.9$ & .7 & 35.7 & 0 & 29.3 & 0.28 \\
27-Jun-94 & ptri & 10 & 30 & 0.40 & 2 & 2 & $20 \pm 19.9$ & .7 & 35.7 & 0 & 31.5 & 0.31 \\
\hline
\end{tabular}

a Lattice type. $b$ Version of program.

have a higher crack strain energy associated with them due to the increase in the magnitude of the crack opening stress. This means that the perturbed lattices will have higher crack strain energy than the unperturbed lattices. This result is confirmed by thermodynamics, because the perturbed lattice has higher entropy and is more stable. Consequently, more strain energy is required to fracture it. This is another manifestation of the observation that higher degrees of randomization require more strain energy to break.

Other implications of this study of lattice perturbation for the triangular lattice are that increasing the heterogeneity in lattice site location causes an increase in the percolation threshold for both homogeneous and heterogeneous site strength distributions and an increase in peak or ultimate strength for heterogeneous site strength distributions. Moreover, increased crack stepping is observed with increased lattice heterogeneity, and for homogeneous site strength, perturbing the lattice geometry affects crack locations immediately, after as few as four sites are cracked, while for the heterogeneous site strength distribution, approximately $15 \%$ of the sites are cracked before perturbation of the lattice geometry begins to affect location of cracked sites. This may indicate the crack density at which geometry begins to dominate site strength in the cracking process. 
The results are preliminary and require further study. However, they do have implications for the design of materials and for analysis of rock and rocklike materials. More detailed analysis is needed to understand exactly when and why crack interaction and geometric effects begin to dominate site strength effects, but this analysis indicates that for materials with homogeneous site strength distributions, lattice geometry begins to dominate immediately.

\subsection{Effect of Heterogeneity in Site Strength: Square Lattice}

In this section results are presented for a study to determine the effect of heterogeneity in site strength for trials computed using a square lattice. This study is similar to that presented for triangular lattices in Section 5.1. Several sets of trials were computed using a square lattice at the same conditions and often with the same input files for site strength and geometry $\left(T_{g^{\prime}}\right.$ $m_{1}$ ) as for the trials on the triangular lattice. Table 5.1-2 summarizes the conditions for which sets of $\mathbf{1 0}$ trials using square lattices were computed. Output parameters for the square lattice trials were the same as for the triangular lattice, including stress-strain, ultimate strength, percolation threshold, normalized crack strain energy, and crack patterns.

\subsubsection{Homogeneous Site Strength}

\section{Stress-Strain}

Stress-strain behavior computed for $10 \times 30$ lattice with confining pressure set at $0.1 \mathrm{MPa}$, $T_{g} 20 \pm .2$, and $m_{1}$ in \pm 0.5 are plotted in Figures 5.5-1 a and $b$. The stress-strain behavior is similar to that observed for the triangular arrays at these conditions. That is, cracking starts at one or two stress levels and continues until a percolating cluster is formed. Figure 5.5-1 a shows that for most of the trials peak stress was followed by strain-softening behavior, and that one trial (\#9) produced much larger strain and stress drop than the others. This is because of the development in this trial of a single, vertical crack that spanned the lattice (see 
discussion in the following section). The behavior of trials $0-8$ is shown in Figure $5.5-1 \mathrm{~b}$ and shows behavior similar to that observed for the triangular lattices as discussed in Section 5.1.1.

\section{Applied Stress vs Normalized Crack Strain Energy}

Figure 5.5-2 presents applied stress vs normalized crack strain energy for this set of trials, and shows that, as expected, cracking of sites for all trials initiated at one or two levels of applied stress. The data for this parameter look very similar to those for the triangular arrays. The values for normalized crack strain energy range form 0.1 to 1.0 , which is a wider range than observed for the triangular lattices.

\section{Number of Cracks vs Normalized Crack Strain Energy}

Data for this parameter are shown in Figure 5.5-3. This plot shows that all trials were similar for only five to seven sites cracked, and as more sites are cracked, values start to diverge and show much wider range of values than for any triangular arrays. The trial with the smallest normalized crack strain energy in trial 1, and examination of the crack patterns shows little crack interaction. The largest normalized crack strain energy is for trial 9. This shows a rapid increase in crack strain energy after 15 to 19 sites are cracked. This may be associated with increased crack interaction.

\section{Crack Patterns}

The development of cracked sites for these trials is illustrated in Figures 5.5-4 a-c, which show locations of broken sites for trial \#9 when 4 and 17 sites are cracked and when the percolation threshold is reached. This shows formation of a cluster of four cracked sites in line (Figure 5.5-4 a), followed by a few dispersed cracked sites (Figure 5.5-4 b) , and then extension of one cluster from top to bottom of the array (Figure 5.5-4 c). The cracked sites break the simulated sample into two columns of material and the stress is transferred to the side columns. This is similar to the behavior observed for triangular lattices in which single linear clusters formed and split the array into two uncracked columns (Section 5.1.1). 
Insight into this behavior can also be gained by observing the crack patterns at failure for all 10 trials, shown in Figure 5.5-5. These crack patterns show that long straight clusters of cracks are formed that are less physically realistic in appearance that those for the triangular lattice. This is due to the precise alignment of the sites on the square lattice. Fracture mechanics analysis shows that the tension in a material is highest directly in front of a crack tip, and for this lattice type, sites are aligned in columns along the direction of maximum principal stress. The cracks link in straight lines, breaking the simulated sample into columns of material. The stress is transferred to the side columns. The loss of stress with small change in strain is due to slabbing. This behavior is also observed in the larger triangular arrays and is discussed further in Section 5.1. It is also interesting to note that as discussed in the preceding section the trial with the smallest normalized crack strain energy is trial 1, and examination of the crack patterns shows low spatial correlation of crack locations, which indicates weak crack interaction. The largest normalized crack strain energy is for trial 9. Crack patterns for this trial show much more spatial correlation and crack interaction after 15 to 19 sites are cracked. Moreover, comparing the crack patterns with the normalized strain energy plots in Figures 5.5-2 and 5.5-3 shows that trials with the straightest cracks produced the highest normalized crack strain energy.

\section{Ultimate Strength and Percolation Threshold}

The mean ultimate strength for these trials was $41.6 \pm 2.0$, and the mean percolation threshold was $0.15 \pm 0.01$. This strength value is similar to that observed for equivalent trials on the triangular lattice, while the percolation threshold is considerably lower than that for

the equivalent trials on a triangular lattice. This is due to the precise alignment of the sites on the lattice that allows long straight cracks to form and propagate while cracking a minimum number of sites. 


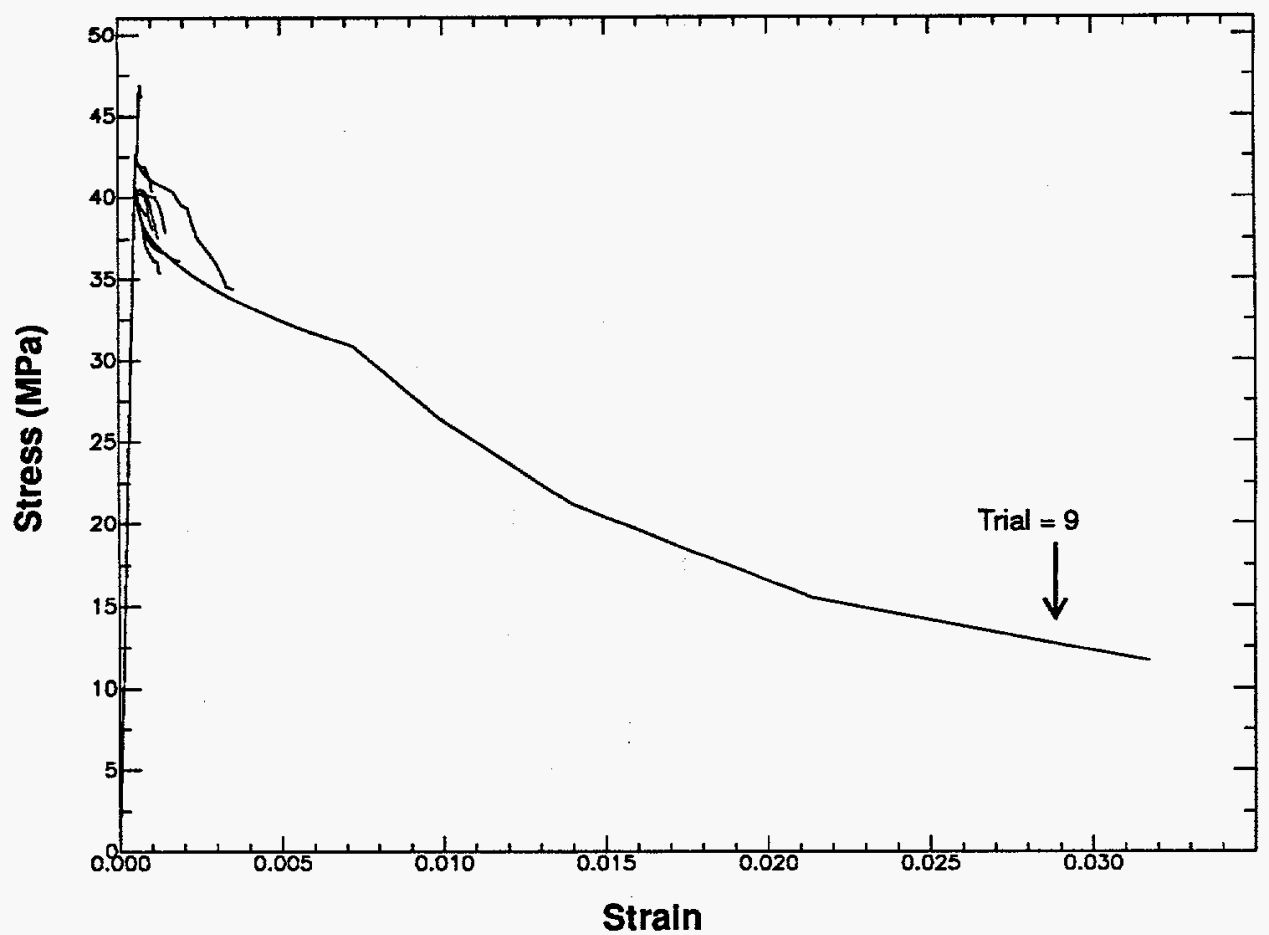

Figure 5.5 - 1a Stress strain curves for 10 trials computed using a square lattice and homogeneous site strength distribution.

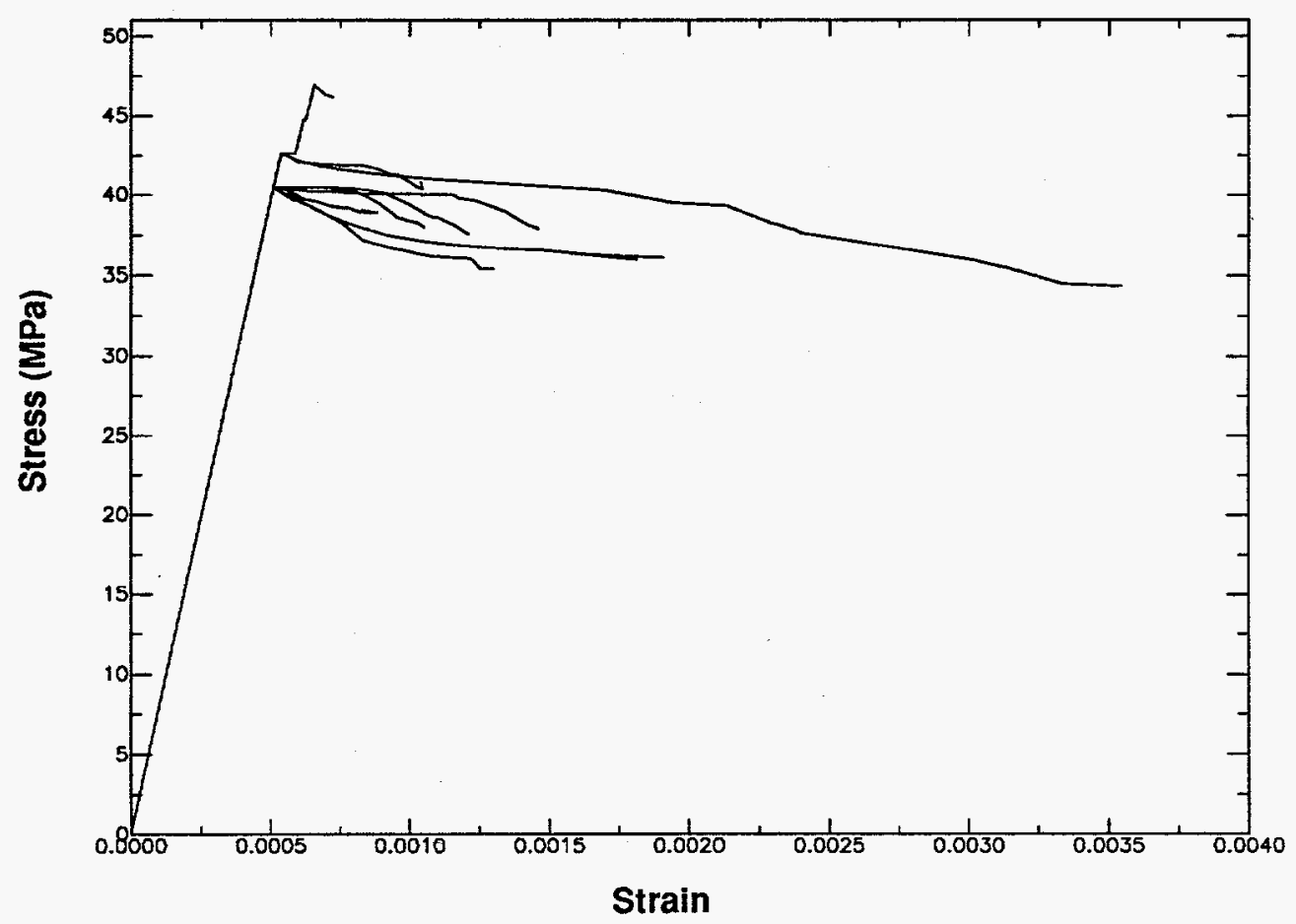

Figure 5.5-1b Stress strain curves for 9 of the trials shown in figure 5.5-1a 


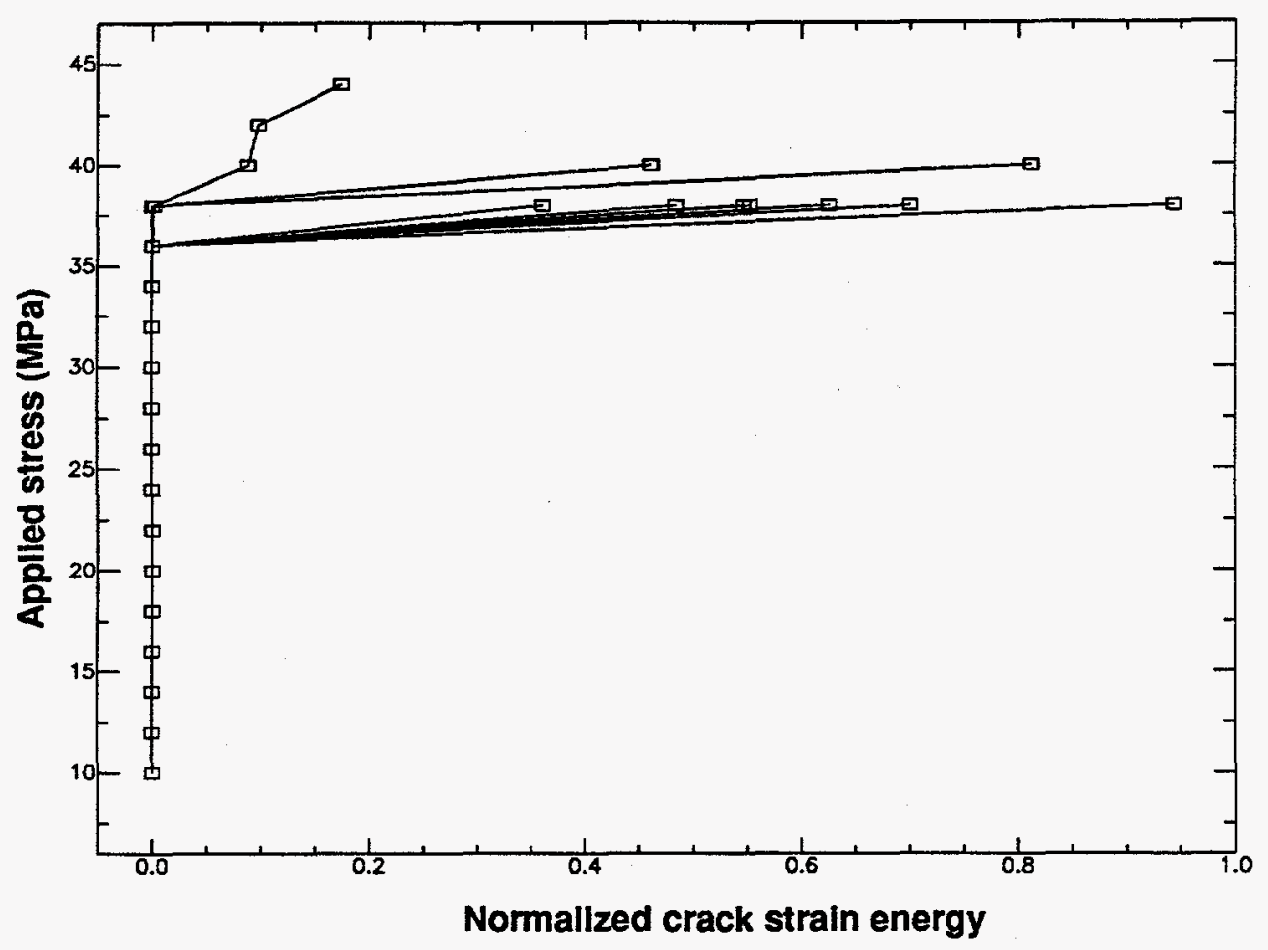

Figure 5.5 - 2 Relation of applied stress to normalized crack strain energy for 10 trials computed using homogeneous site strength distributions on a square lattice.

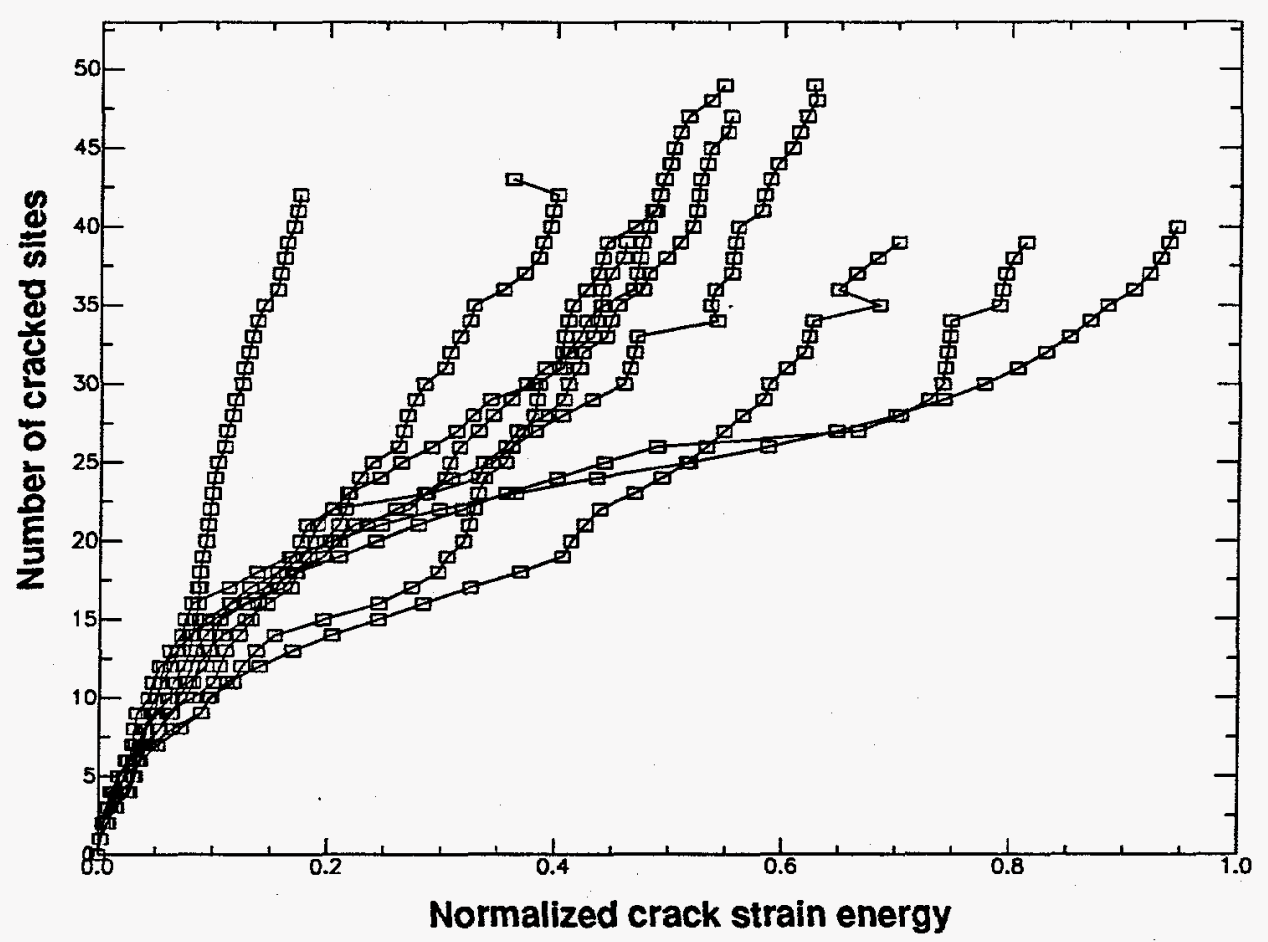

Figure 5.5 - 3 Relation of number of cracked sites to normalized crack strain energy for 10 trials computed using a homogeneous site strength distributions on a square lattice 


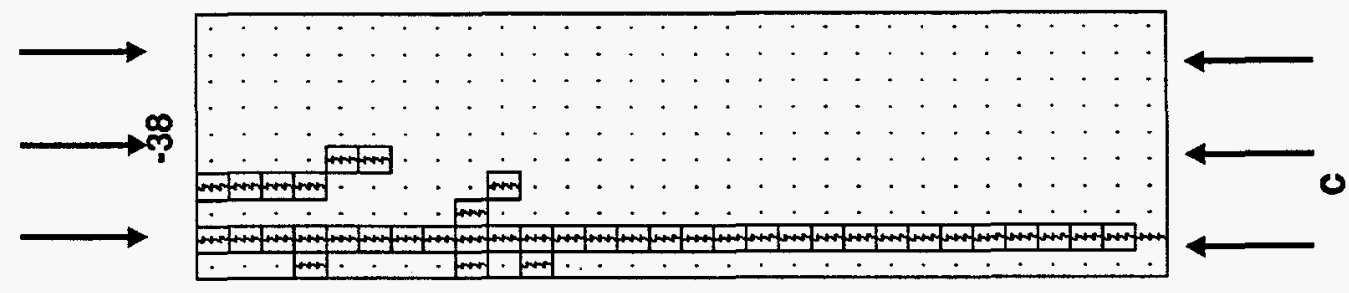

동
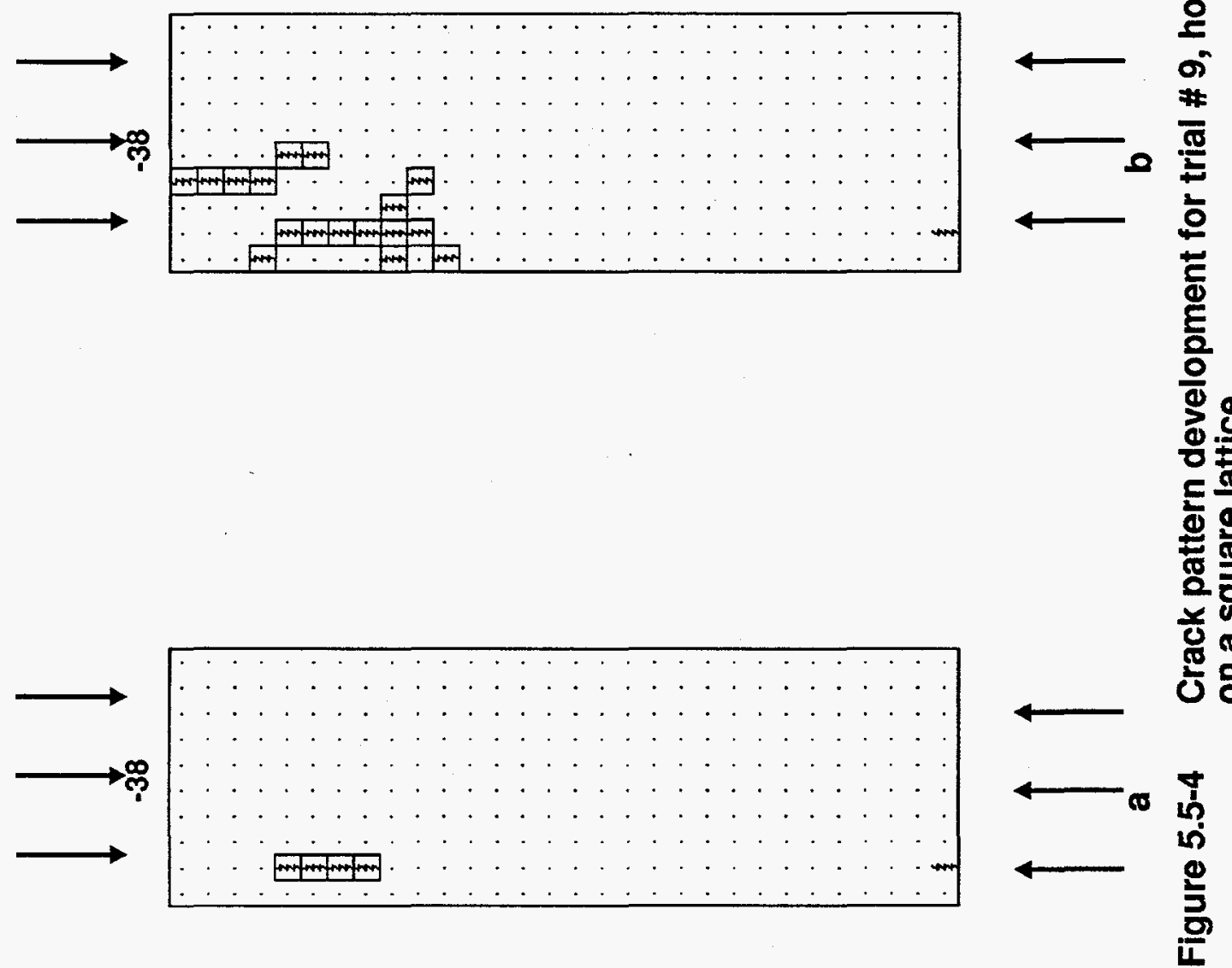

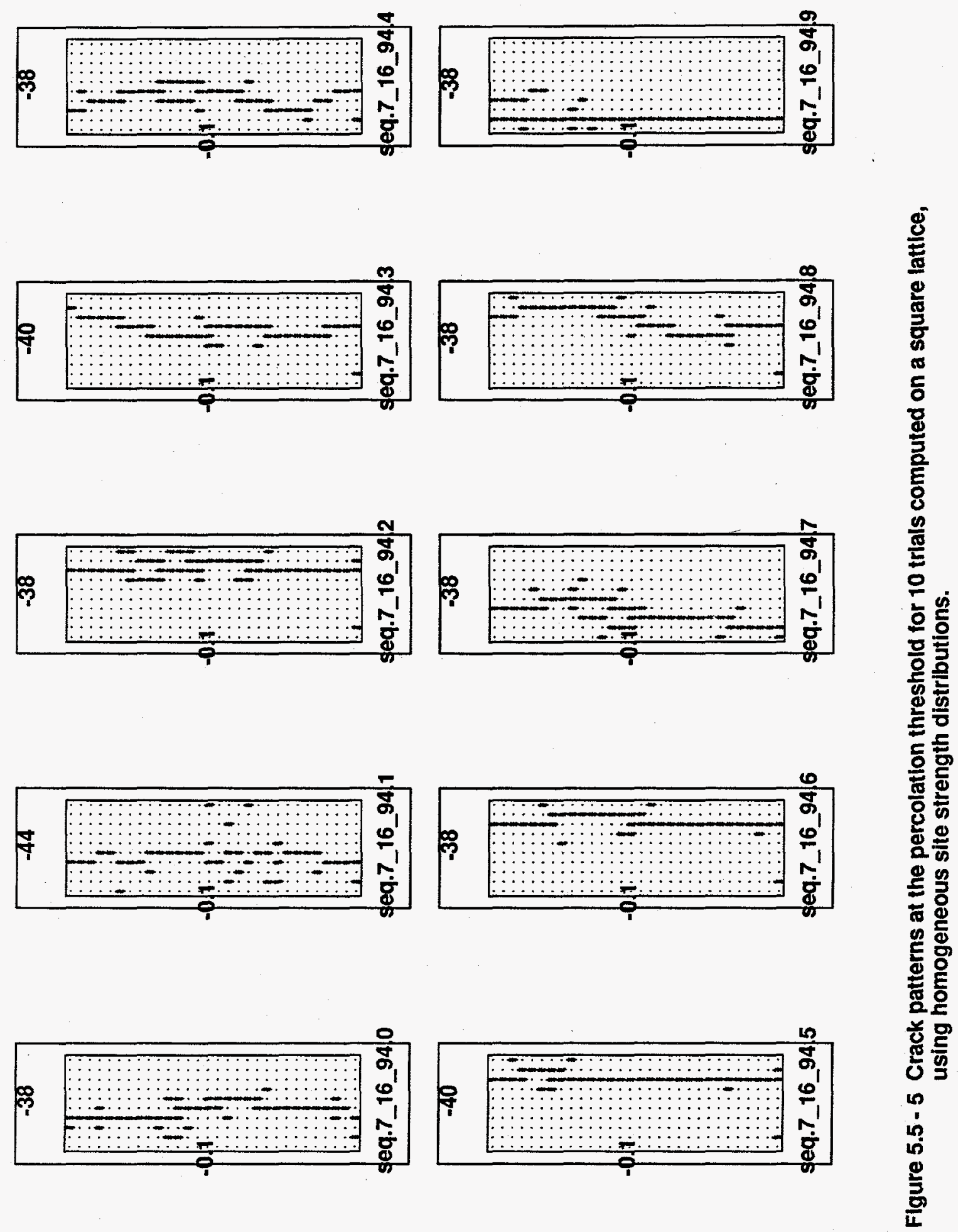


\subsubsection{Heterogeneous Site Strength}

Trials were also computed using square arrays and heterogeneous site strength distributions. The site strength distributions and values of $m_{1}$ were the same data sets as were used for the triangular lattice discussed in Section 5.1.2.

\section{Stress-Strain}

Stress-strain curves for the 10 trials are shown in Figure 5.5-6. As expected these show a wider distribution in peak strength than the trials with homogeneous site strength. Also, nonlinear behavior is observed at lower levels of applied stress. Trials form two groups, with a group showing peak stress in the range $30-35 \mathrm{MPa}$ and a group with peak strengths in the range 40-47 MPa. Many of the curves show sequential intervals of strain-hardening/strainsoftening/strain-hardening.

\section{Applied Stress vs Normalized Crack Strain Energy}

Plots of applied stress vs normalized crack strain energy are shown in Figure 5.5-7. This figure shows the grouping of the trials into two sets more dramatically than the stress-strain curves, and shows that the normalized crack strain energy is larger for the weaker arrays, indicating that increased crack interaction may lower the ultimate stress, and conversely higher stresses are required when crack interaction is repressed. The range of values of normalized crack strain energy is similar to that observed for triangular arrays, indicating that the occurrence of very long straight cracks is suppressed by the increased heterogeneity.

\section{Number of Cracks vs Normalized Crack Strain Energy}

Figure 5.5-8 shows the values of number of cracked sites vs. normalized crack strain energy for these trials. All of the curves are very similar and are tightly bunched up to about 30 cracked sites. A major change in slope occurs at approximately 45 cracked sites. The curves are not as widely divergent as are those for homogeneous site strength distributions. 


\section{Crack Patterns}

The development of the crack pattern for trials 7 and 8 is shown in Figures $5.5-9 a$ and $b$. These trials represent the weaker and stronger subsets of the trials at this condition respectively. Both show that the initial population of sites that crack are spatially uncorrelated. The subsequent locations of cracked sites in trial 7 (Figure 5.5-9a) show more spatial correlation, resulting in one thin percolating cluster that has a few side steps. The crack pattern for trial 8 (Figure 5.5-9b) shows weaker spatial correlation of cracked sites as the cracking progresses. This may be the result of crack arrest due to a very strong site, or due to the formation of a split cluster in the right central region of the array.

Locations of cracked sites at the percolation threshold for all 10 trials are shown in Figure 5.5-10. This figure shows that the locations of cracked sites are much more distributed than for the homogeneous site strength case on the square lattice. The formation of long straight cracks is shown in trials 0 and 7 , and this may contribute to the lower strength observed for these trials. This figure also shows that for the square arrays, clusters are much more linear and controlled by the lattice structure than are the clusters observed for triangular lattices.

\section{Ultimate Strength and Percolation Threshold}

The mean ultimate strength for these trials was $38.8 \pm 5.18 \mathrm{MPa}$, which is lower than that observed for the heterogeneous triangular and homogeneous square trials. Mean percolation threshold was $0.25 \pm 0.026$. This is higher than the homogeneous site strength distribution on the square lattice, but lower than the value for the heterogeneous site strength distribution on the triangular lattice.

\subsubsection{Summary of Site Strength Study on Square Lattice}

Summary data for peak strength and percolation threshold for trials on square lattices are given in Table 5.5-1. Results show that for the square lattice, increasing heterogeneity is site strength lowered the peak strength and increased the percolation threshold. This is in contrast to the results for the triangular lattice, which showed no change in peak strength with 


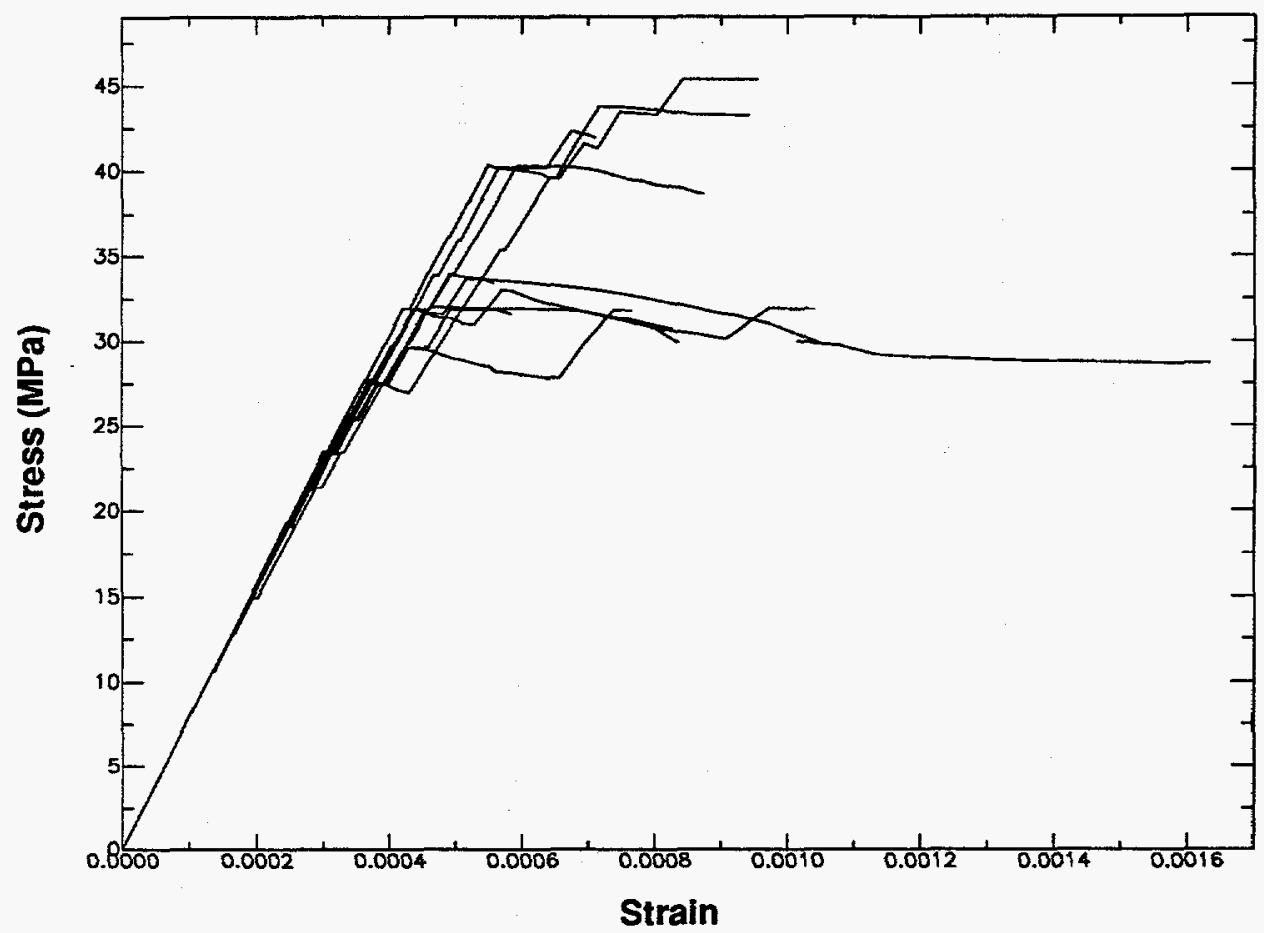

Figure 5.5 - 6 Stress-strain curves for 10 trials computed using heterogeneous site strength distributions on a square lattice.

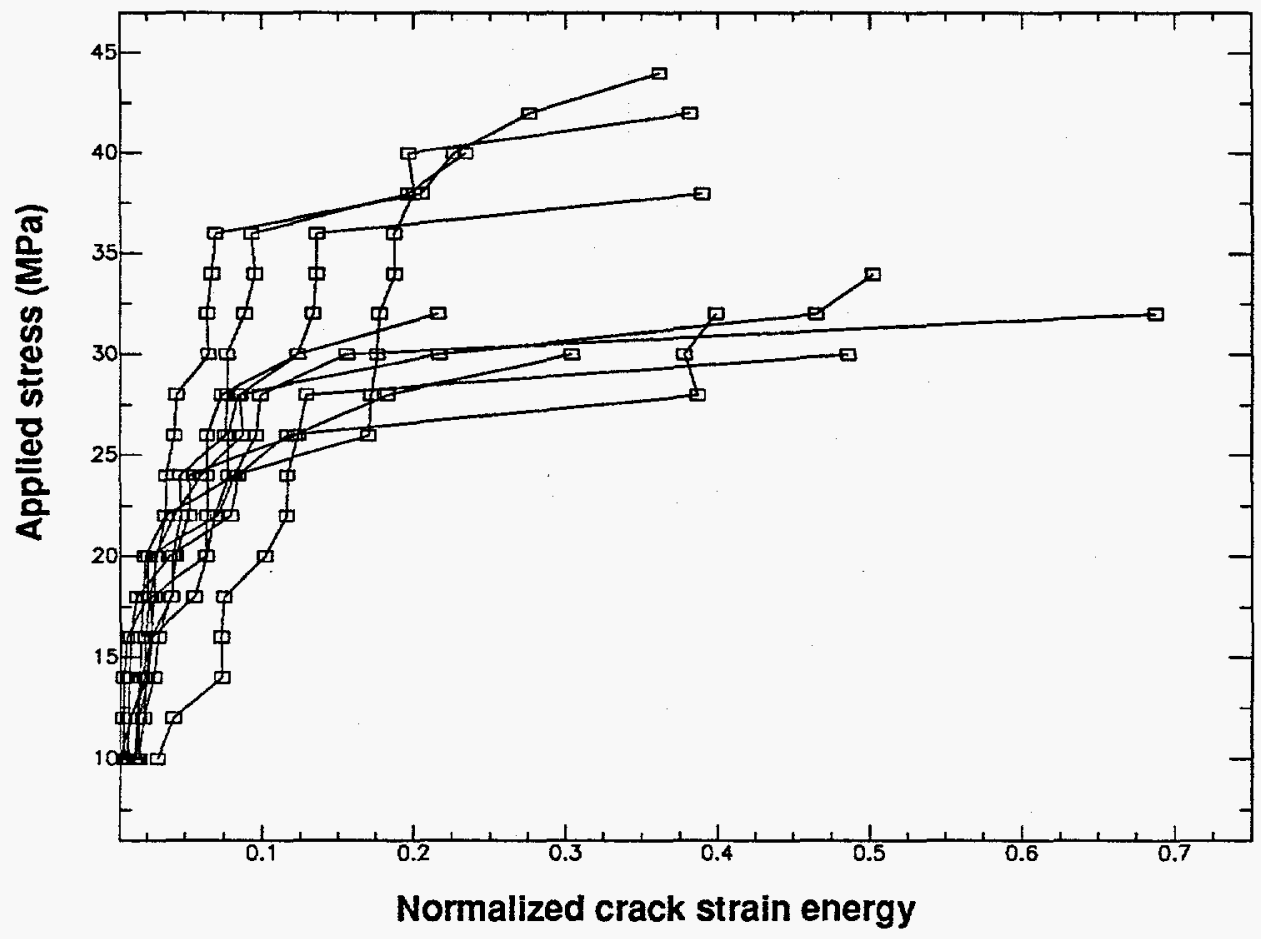

Figure 5.5 - 7 Relation of applied stress to normalized crack strain energy for 10 trials computed using heterogeneous site strength distributions on a square lattice. 


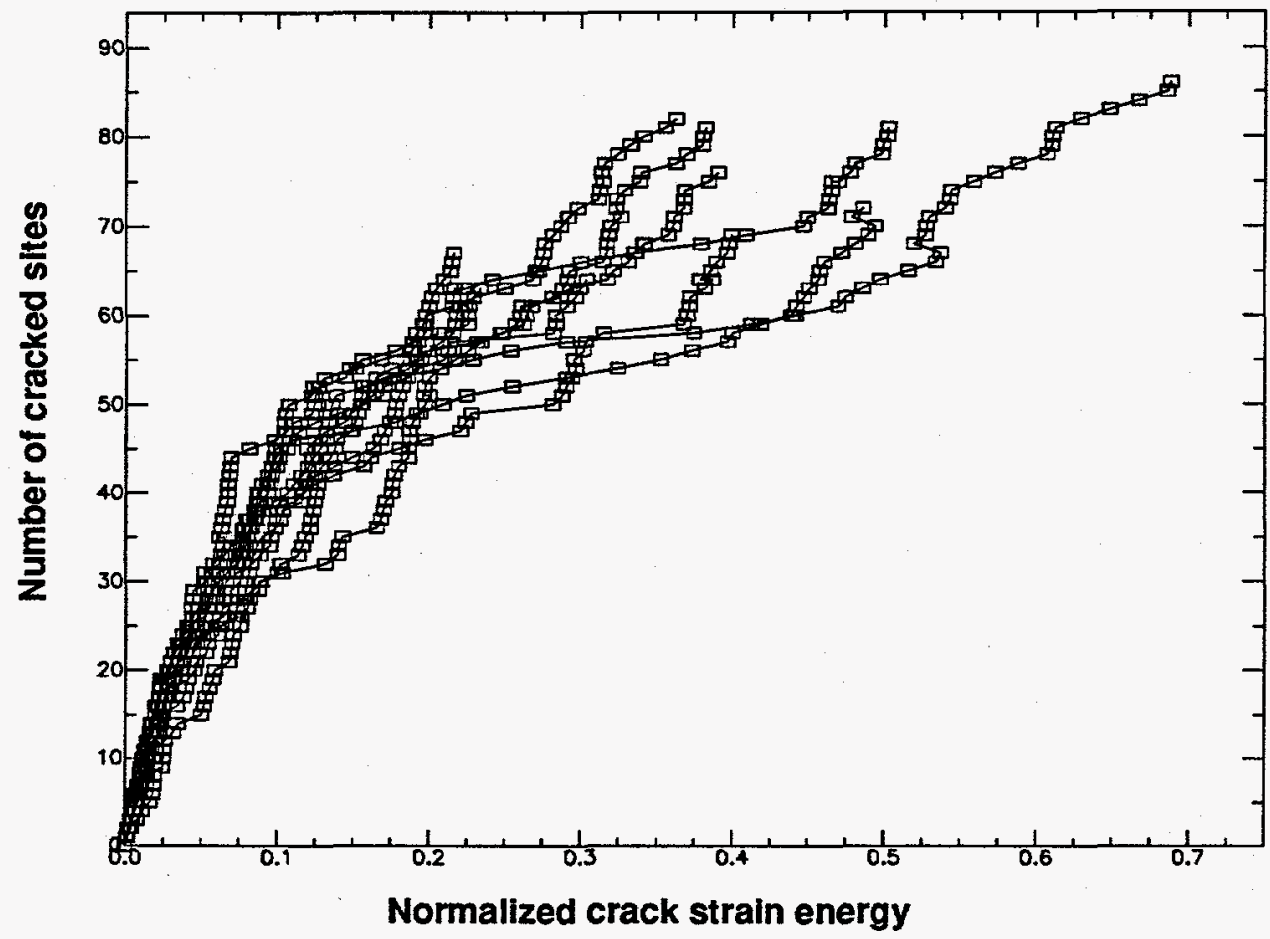

Figure 5.5 - 8 Relation of number of cracked sites to normalized crack strain energy for 10 trials computed using heterogeneous site strength distributions on a square lattice. 

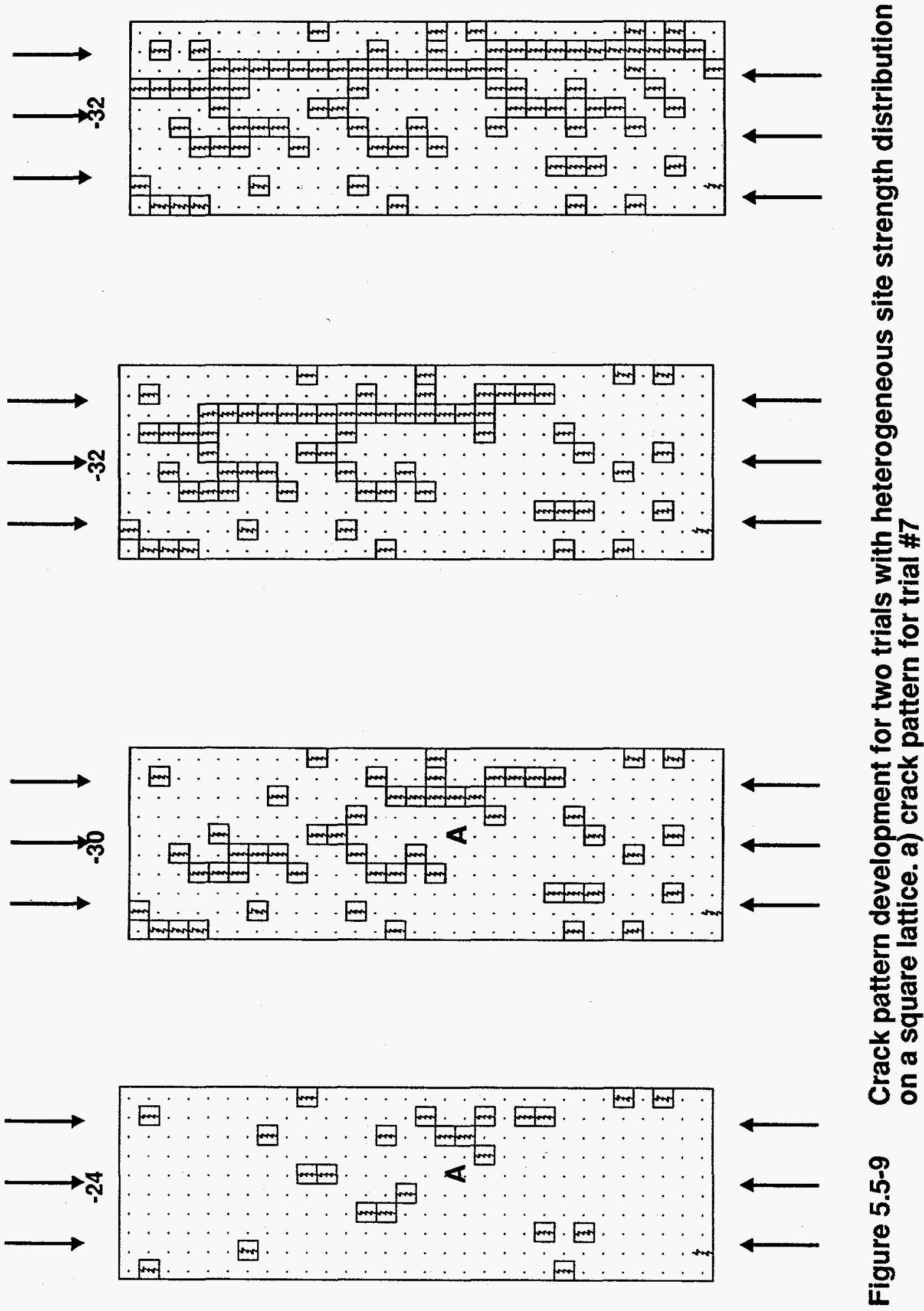

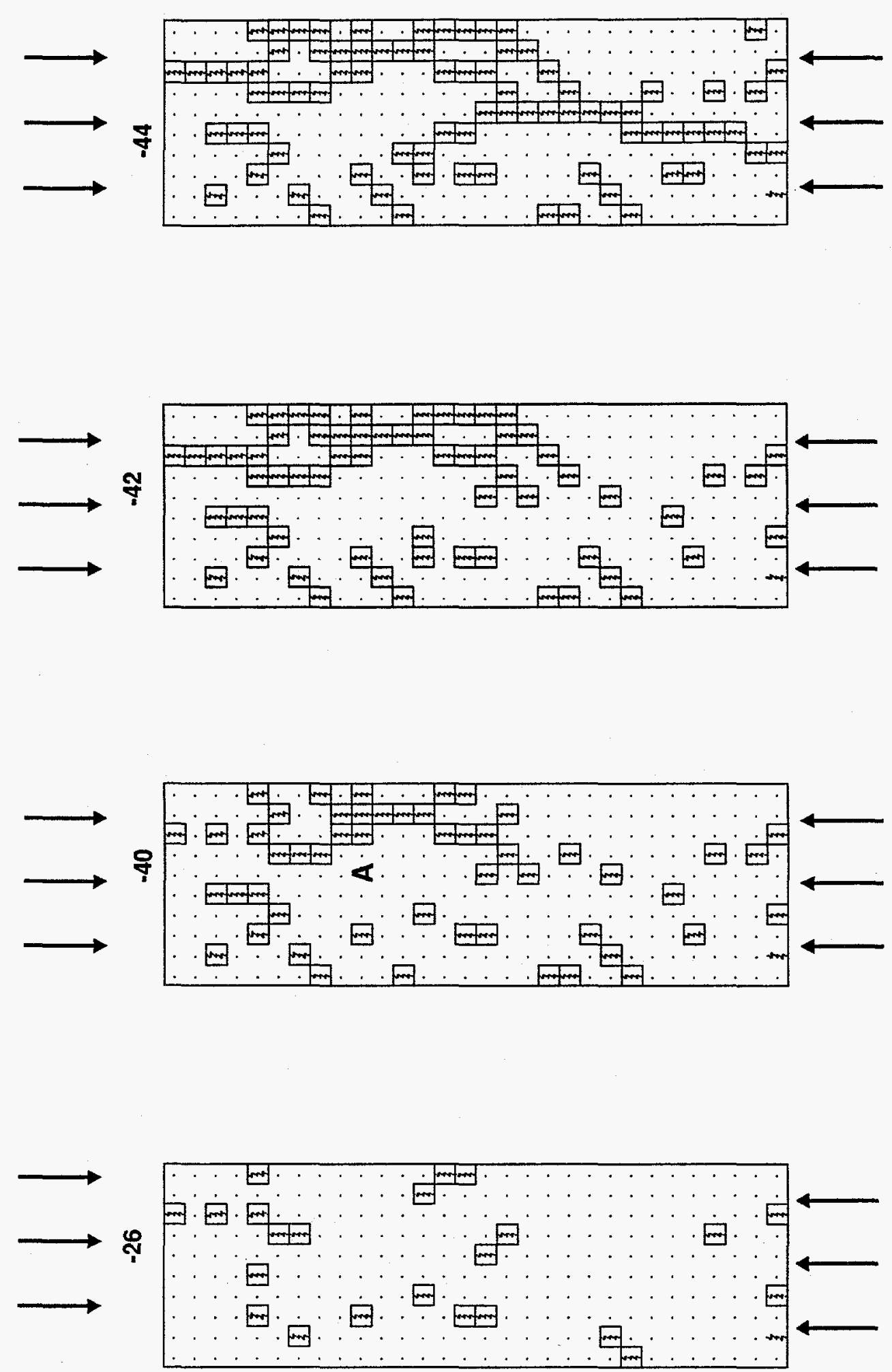

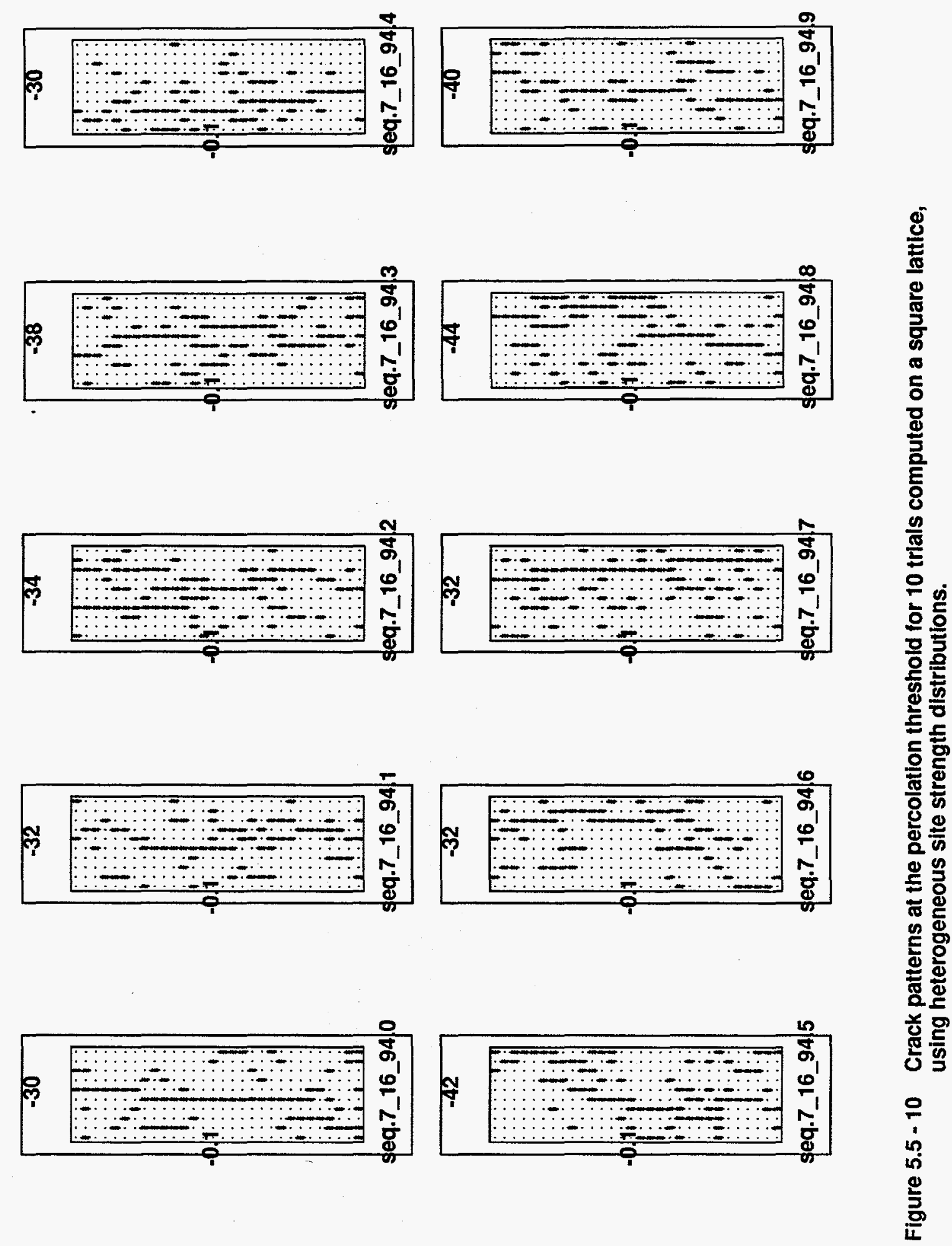
Table 5.5-1. Summary data for square lattices.

\begin{tabular}{ccc}
\hline Site strength & Peak stress & Percolation threshold \\
\hline $20 \pm .2$ & $41.6 \pm 2.0$ & $0.15 \pm 0.01$ \\
$20 \pm 19.9$ & $36.8 \pm 5.2$ & $0.25 \pm 0.03$ \\
\hline
\end{tabular}

increasing site strength heterogeneity. This may be because on the square lattice, stronger crack interaction occurs.

For heterogeneous site strength distributions the range of values of normalized crack strain energy is similar to that observed for triangular arrays, indicating that the occurrence of very long straight cracks is suppressed by the increased heterogeneity.

\subsection{Effect of Stress Heterogeneity on Square Lattice}

The purpose of this section is to show how changing the range of the $m_{1}$ parameter affects the macroscopic behavior for trials on a square lattice. Trials with homogeneous and heterogeneous site strength distributions were computed with $m_{1}$ in the ranges $\pm 0.3, \pm 0.5$, and \pm 0.7 . The mean peak strength for the trials was again used as a measure of the macroscopic behavior. The results for the square arrays are summarized in Table 5.6-1 and are plotted in Figure 5.6-1 along with results presented previously for the triangular lattice. This figure shows that the mean ultimate strength computed for the $10 \times 30$ square lattices over the range of conditions evaluated was very similar to that computed for the triangular lattices. This shows that the site geometry dominates the behavior on the square lattice. This is consistent with the observations for the triangular lattices presented in Section 5.2.

Moreover, the percolation thresholds for trials on the square lattices are lower. For homogeneous site strength the percolation thresholds are significantly lower than for the triangular lattices. Again, this is due to the precise alignment of the sites, locating them in the 


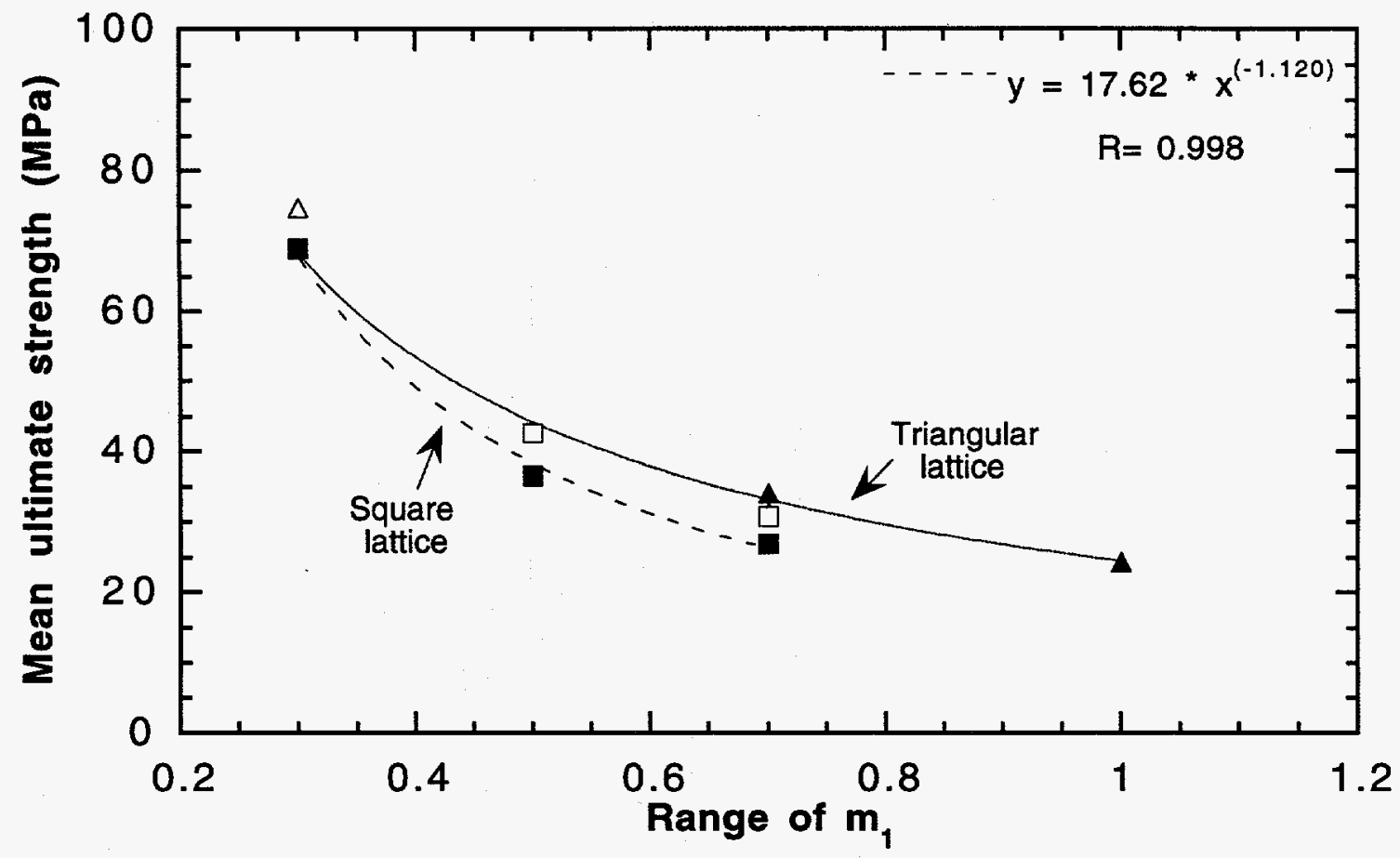

Figure 5.6-1. Dependence of ultimate strength on $m_{1}$ for square and triangular lattices. Open and solid symbols represent homogeneous and heterogeneous site strength distributions, respectively. 
Table 5.6-1. Summary of trials for square arrays.

\begin{tabular}{clccccc}
\hline Trial date & strength & \multicolumn{1}{c}{$\begin{array}{c}\sigma_{\mathrm{I}} \\
\text { snitial }\end{array}$} & $m_{1}$ & $\Delta \sigma_{\mathrm{I}}$ & \multicolumn{1}{c}{$\sigma_{\mathrm{pk}}$} & \multicolumn{1}{c}{$p_{c}$} \\
\hline 11_24_93 & $20 \pm 19.9$ & 10 & .3 & 10 & $69.1 \pm 12.5$ & $0.29 \pm 0.03$ \\
11_24_93 & $20 \pm .2$ & 10 & .5 & 10 & 42.7 & $0.14 \pm 0.02$ \\
11_24_93 & $20 \pm 19.9$ & 10 & .5 & 10 & $36.6 \pm 5.9$ & $0.26 \pm 0.05$ \\
2_25_94 & $20 \pm .2$ & 10 & 0.7 & 10 & $33.9 \pm 3.9$ & $0.17 \pm 0.06$ \\
2_25_94 & $20 \pm 19.9$ & 10 & 0.7 & 10 & $31.4 \pm 1.4$ & $0.26 \pm 0.03$ \\
6_28_94 & $20 \pm .2$ & 2 & 0.7 & 2 & $30.8 \pm 1.5$ & $0.16 \pm 0.04$ \\
6_28_94 & $20 \pm 19.9$ & 2 & 0.7 & 2 & $27.0 \pm 4.7$ & $0.27 \pm 0.04$ \\
\hline
\end{tabular}

optimum place for maximizing the stress intensity factor. Overall crack density is lower due to the nature of the lattice, strengths are similar, and percolation thresholds are lower.

\subsection{Perturbed Square Lattice}

This section presents results for trials computed to evaluate the effect of perturbing the site locations of a square lattice. The general methodology for lattice perturbation has been presented in Sections 4.3 and 5.4 and will not be repeated here. This section follows the structure of Section 5.4 by presenting results for homogeneous and heterogeneous site strength distributions on unperturbed square lattices and perturbed square lattices. For the perturbed lattices, radii in the range $0 \leq r \leq 0.04 l_{c}$ and $0 \leq r \leq 0.4 l_{c}$ were used. The amount of lattice perturbation is denoted using r.00, r.04, and r.40, respectively, as in Section 5.4. Trials computed using the perturbed lattice sites used the same input files of site strength and $m_{1}$. In this manner the effect of increasing the amount of heterogeneity in site location is determined.

\subsubsection{Homogeneous Site Strength}

\section{Stress-Strain}

Stress-strain behavior for all three trials is shown in Figure 5.7-1 and is similar for all trials, with r.00 showing a step in stress while r.04 and r.40 do not. 


\section{Applied Stress vs Normalized Crack Strain Energy}

Values for applied stress are plotted vs normalized crack strain energy in Figure 5.7-2. This figure shows that for trials r.04 and r.40 all sites cracked at an applied stress of $30 \mathrm{MPa}$. Values for r.00 show that sites cracked at two different levels of applied stress. Values of normalized crack strain energy are similar for all trials.

\section{Number of Cracks vs Normalized Crack Strain Energy}

Computed values for these parameters are shown in Figure 5.7-3. This plot shows that for the first 18 cracked sites behavior of these parameters is similar for all three trials. After 18 sites have cracked the values for r.00 diverge. Values for r.04 and r.40 are similar up to 30 sites cracked; then r.40 shows smaller gains in normalized crack strain energy with sites cracking. This figure is similar to that observed for the perturbed triangular lattice at these conditions, in that all are similar up to approximately 15 cracks, then the lattice that is most perturbed requires more cracks to achieve the same level of normalized crack strain energy. Also, the curves for r.04 and r.40 are similar to each other and differ from the curve for r.00.

\section{Crack Patterns}

The sequence of cracking for these trials is shown in Figures 5.7-4 a-o. Figures $5.7-4 a, b$, and c show identical sites cracked for up to 8 cracked sites; Figures $5.7-4 \mathrm{~d}$, e, and f show that at 18 cracked sites the pattern is similar for each trial, with cluster A having extended downward in all trials, but maintaining an overall vertical orientation in r.00, while the general trend in r.04 and r.40 is trending right to left. Figures $5.7-4 \mathrm{~g}, \mathrm{~h}$, and $\mathrm{i}$ show the pattern for 30 cracked sites. This figure shows that for r.00, cluster $A$ has stopped and 5 new small clusters have formed. In contrast, the patterns for r.04 and r.40 show that cluster A has continued to extend downward. At this stage all clusters are connected to the top of the array. Figures 5.7-4 $j, k$, and 1 show the pattern for 40 cracked sites. This figure shows that for $\mathrm{r} .04$ a critical cluster has formed, and $\mathrm{r} .40$ has a cluster that nearly spans the array. In contrast, a new cluster $\mathrm{C}$ has formed in r.00. 


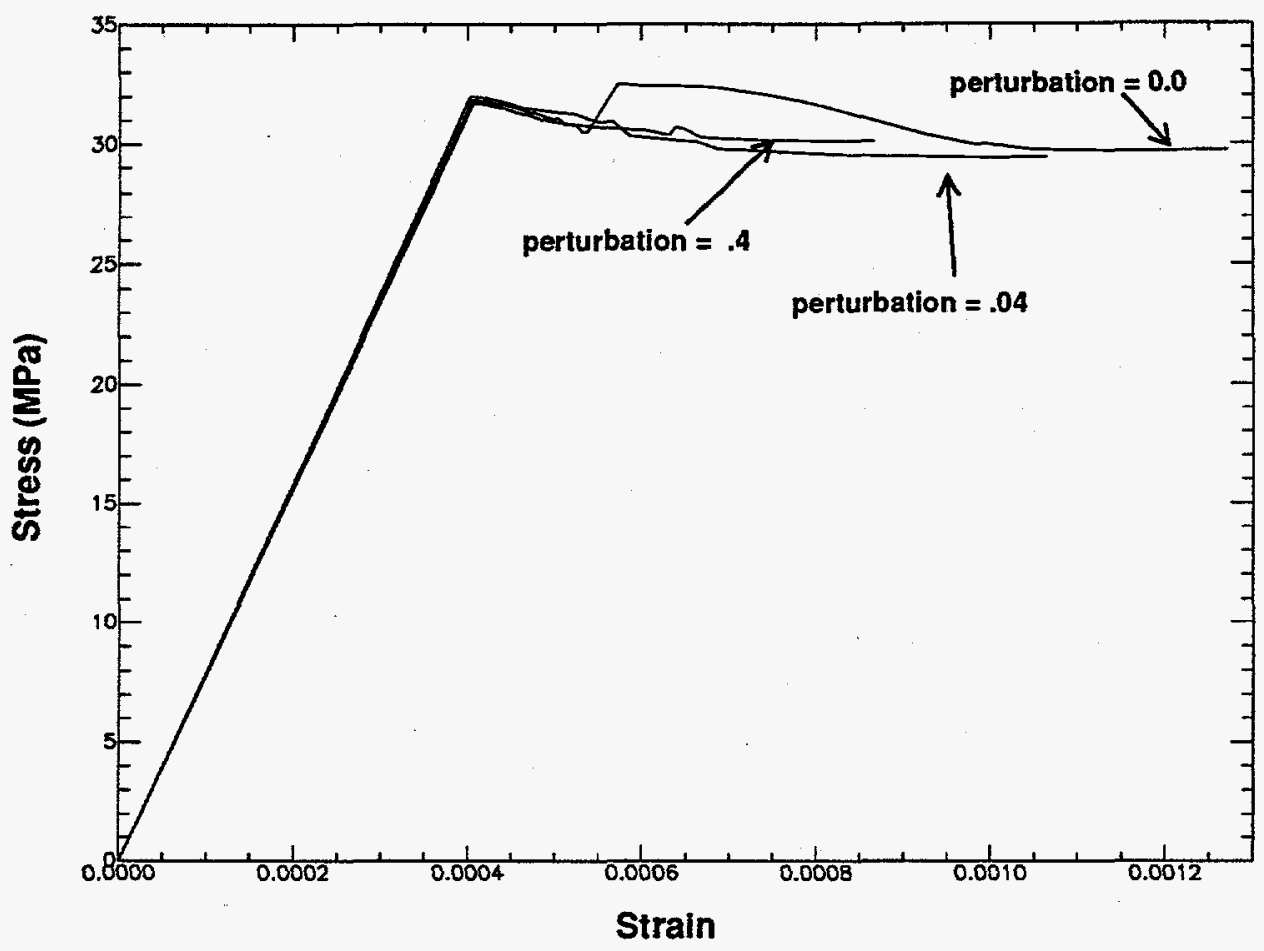

Figure 5.7-1 Effect of perturbation of a square lattice on stress-strain curves for trials computed using a homogeneous strength distribution.

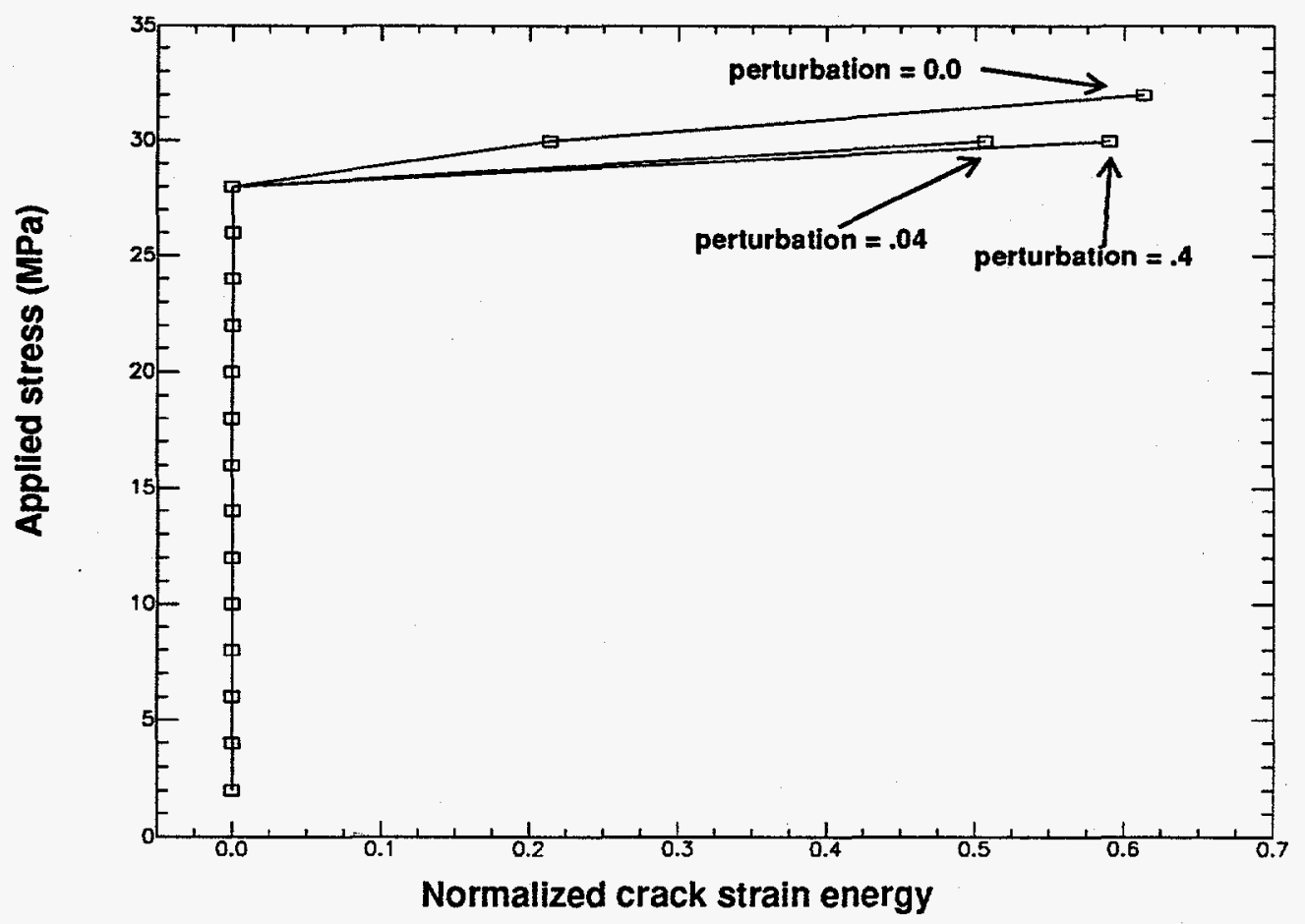

Figure 5.7 - 2 Effect of perturbation of a square lattice on relation of applied stress to normalized crack strain energy for trials computed using a homogeneous site strength distribution. 


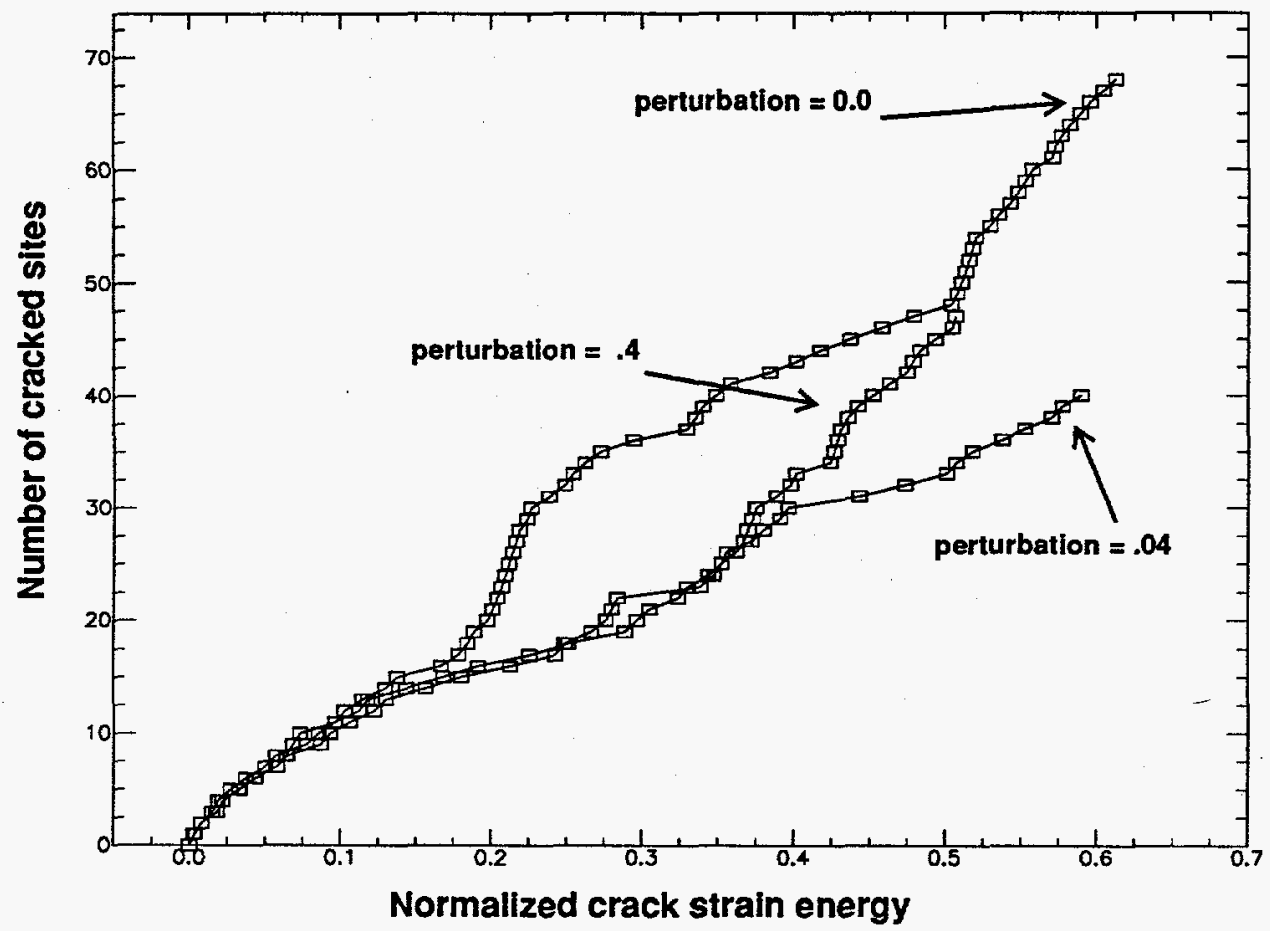

Figure 5.7-3 Effect of perturbation of a square lattice on relation of number of cracked sites to normalized crack strain energy for trials computed using homogeneous site strength distribution. 


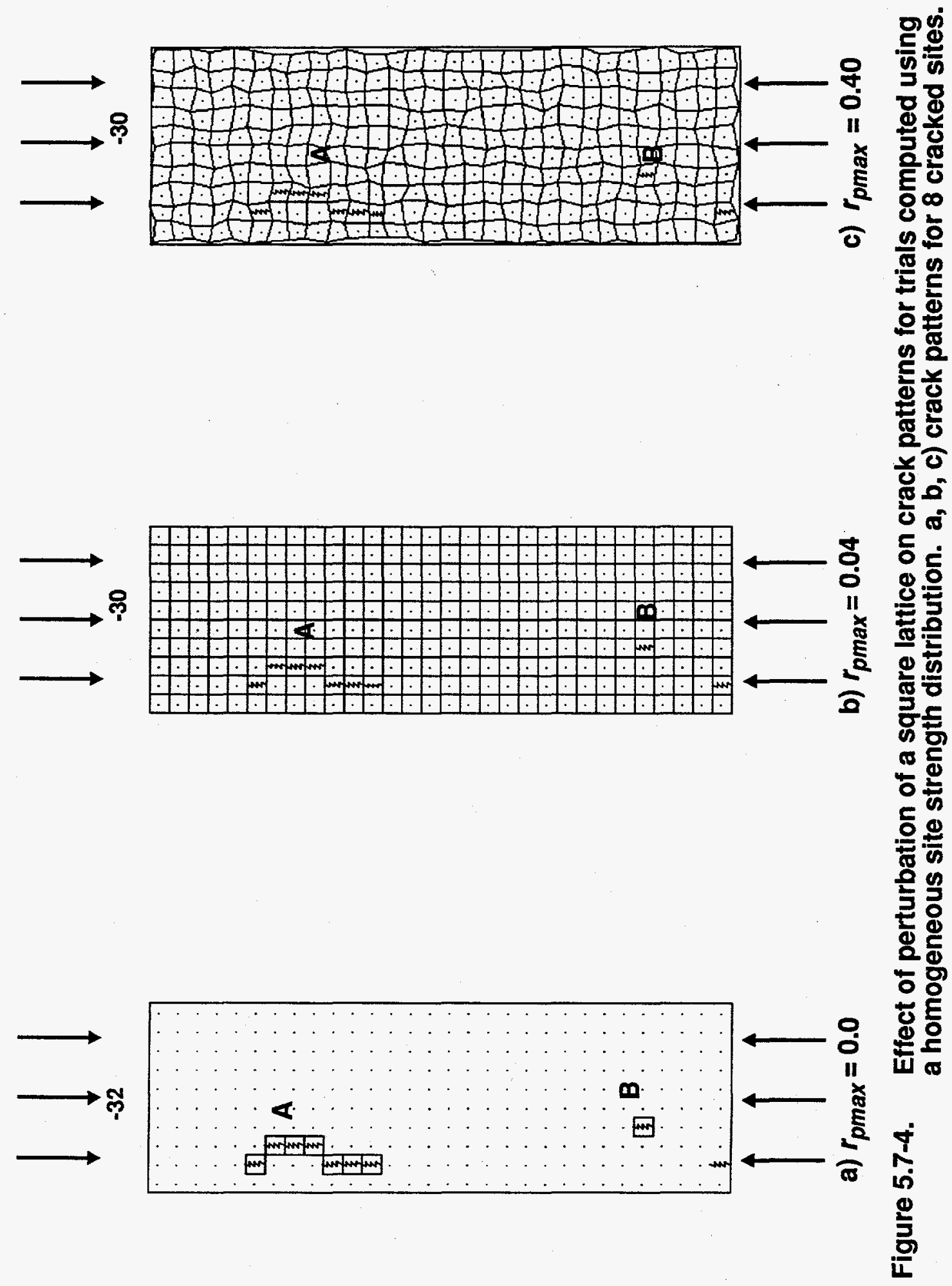



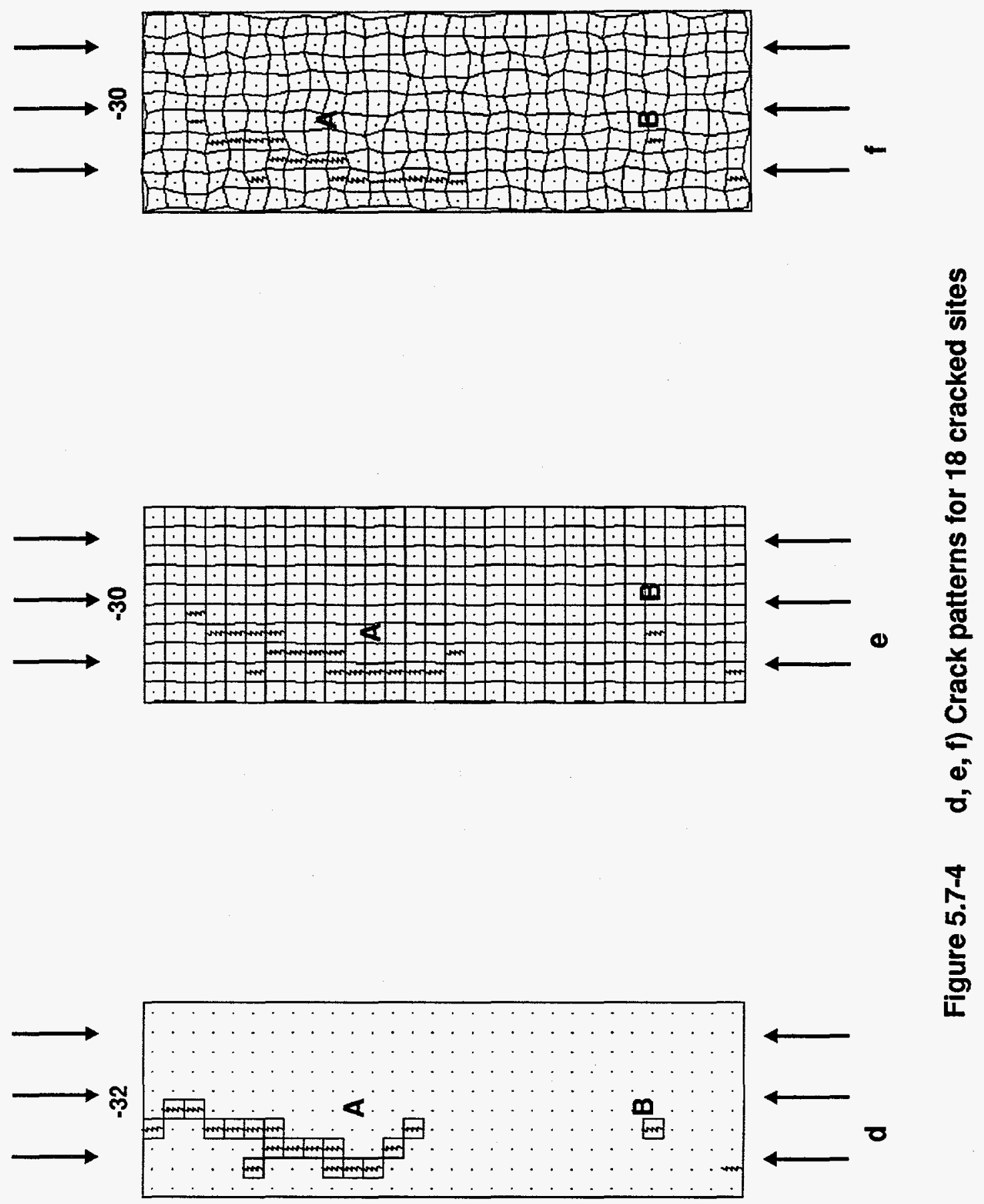

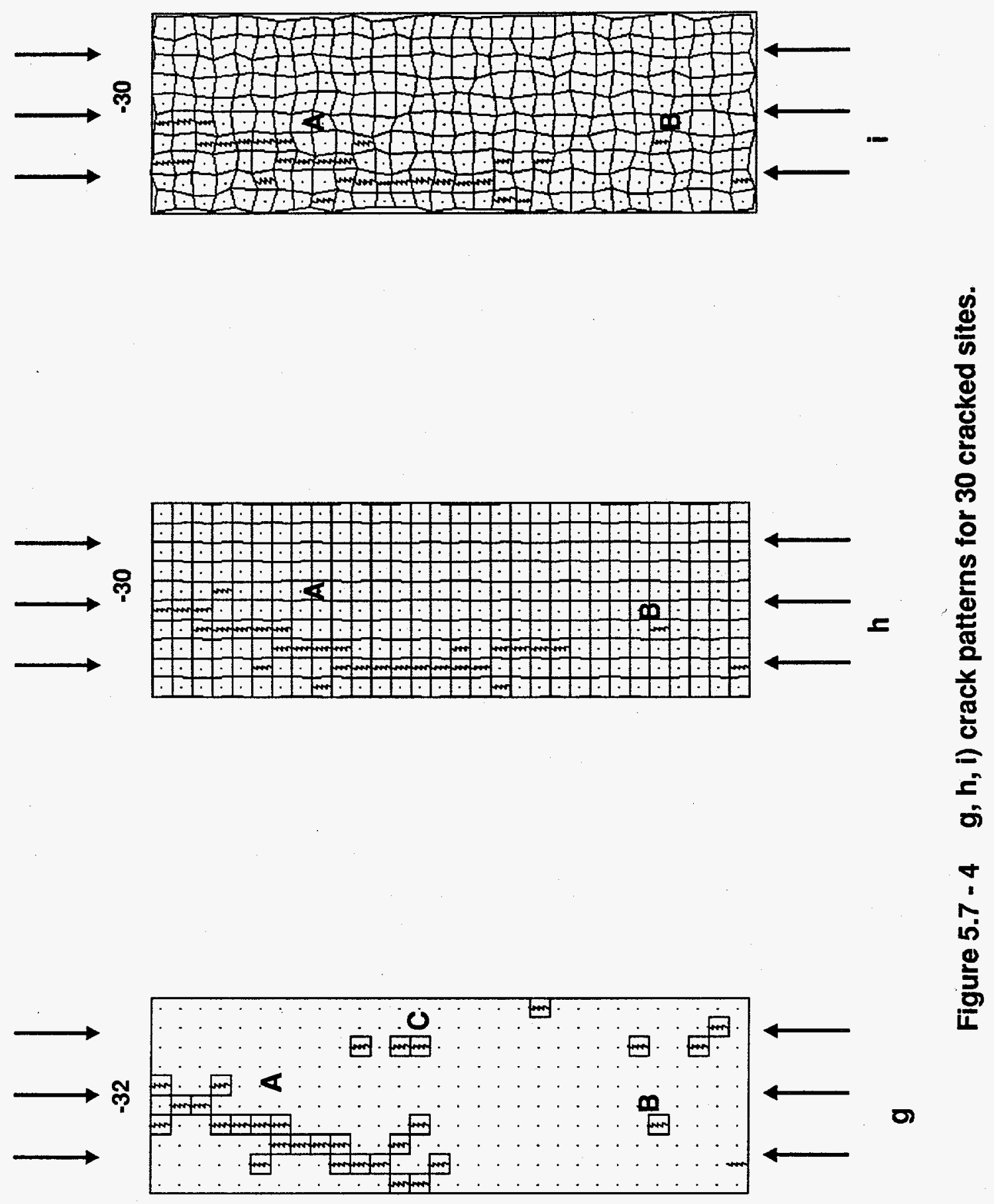

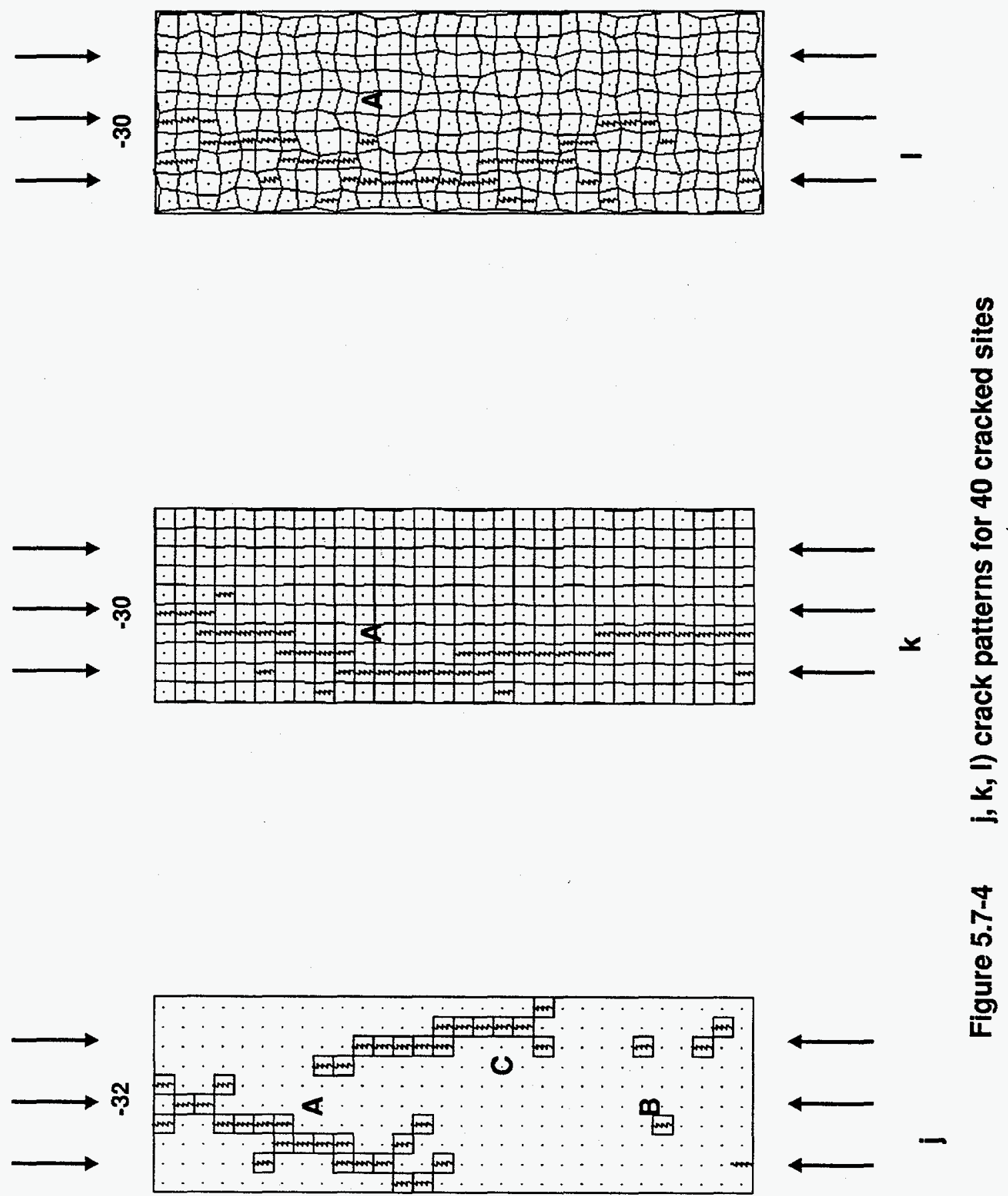

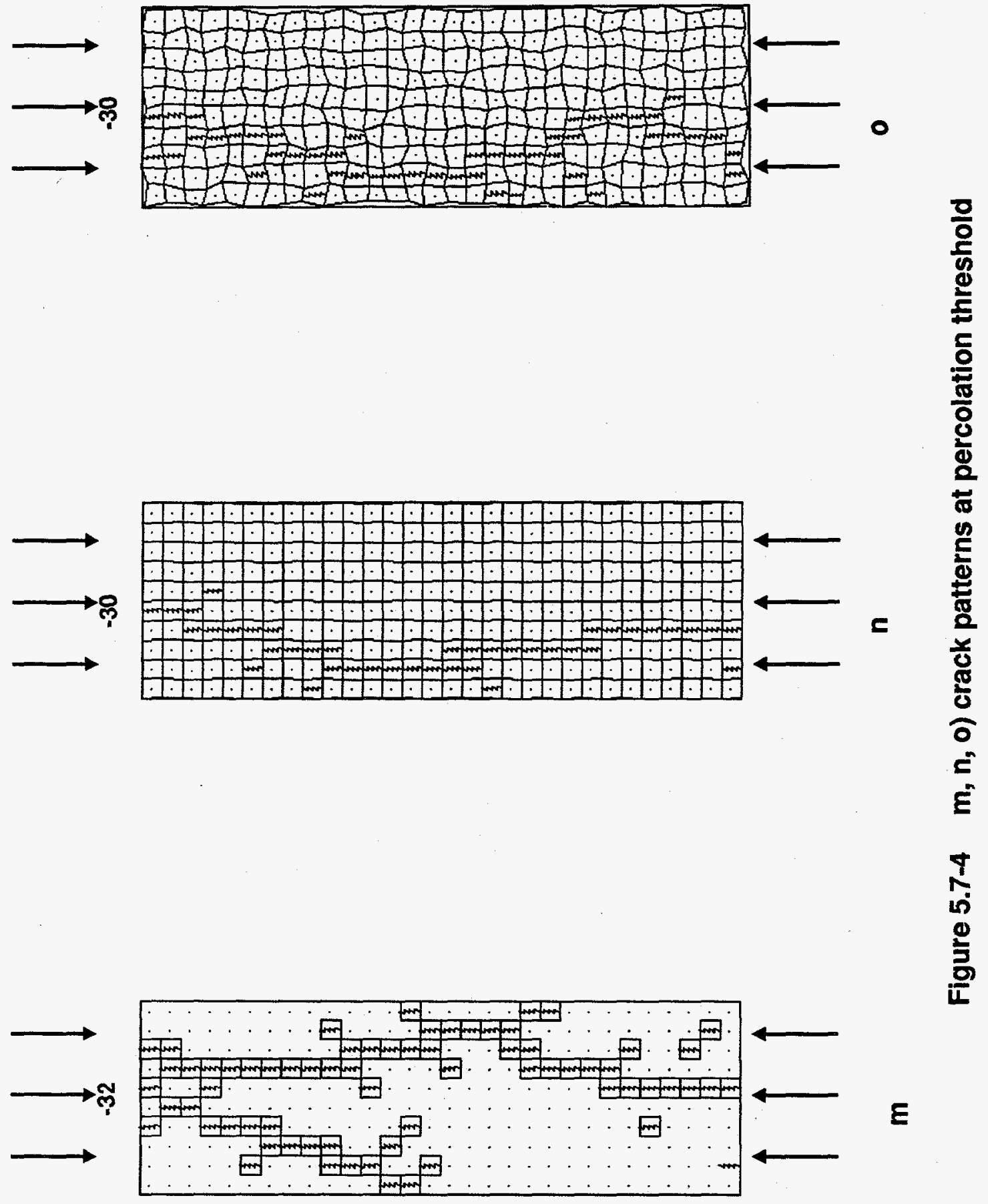
The pattern of cracked sites at formation of the critical cluster is shown for all three trials in Figures $5.7-4 \mathrm{~m}, \mathrm{n}$, and o. A big difference is shown between $\mathrm{r} .00$ and r.04, while the patterns for r.04 and r.40 are very similar. This is consistent with the observations for the perturbed triangular lattice, which also showed that the behavior was similar for perturbed cases and is different from the unperturbed case.

\section{Discussion}

Perturbation of the square lattice for a homogeneous site strength distribution showed identical patterns of cracked sites up to eight sites cracked. Patterns diverge at 18 cracked sites, and r.04 and r.40 were similar up to 30 sites cracked. This behavior is similar to triangular homogeneous trials that have an early difference in r.00, r.04, and r.40, and the perturbed lattices show similar behavior for most of the loading sequence.

\subsubsection{Heterogeneous Site Strength}

Trials for perturbed triangular lattices using a heterogeneous site strength distribution were also computed. Results of these trials are discussed below.

\section{Stress-Strain}

Stress-strain curves for these trials are presented in Figure 5.7-5. These curves are very similar to each other up to a stress of $25 \mathrm{MPa}$. Above $25 \mathrm{MPa}$ the curve for unperturbed (r.00) is similar to that for the r. 04 curve and shows strain hardening. The r. 40 curve strain softens after $25 \mathrm{MPa}$.

\section{Applied Stress vs Normalized Crack Strain Energy}

Values computed for these parameters are shown in Figure 5.7-6. All curves are similar up to $25 \mathrm{MPa}$ where r.00 and r.04 show little change in normalized crack strain energy with applied stress in contrast to the r.04, which continues to form cracks. 


\section{Number of Cracks vs Normalized Crack Strain Energy}

Data for these parameters are plotted in Figure 5.7-7. All curves are similar up to approximately 80 cracks, where r.40 shows an increase and stops with fewer cracks.

\section{Crack Patterns}

Crack patterns for the three trials when 25,45 , or 80 sites are cracked and at the formation of the critical cluster are shown in Figures 5.7-8 a-1. Figures 5.7-8 a, b, and $c$ show that when 25 sites are cracked the patterns are nearly identical. Figures $5.7-8 \mathrm{~d}, \mathrm{e}$, and $\mathrm{f}$ show that when 45 sites are cracked r.00 and r.04 are still similar, but r.40 has diverged slightly. When 80 sites are cracked (Figures $5.7-8 \mathrm{~g}, \mathrm{~h}$, and i) all patterns are still similar. Crack patterns at the percolation threshold for r.00 and r.04, shown in Figures 5.7-8 j, k, and 1, are very similar, and different from the curve for $r .40$. This is in contrast to the previous results for crack patterns.

The behavior is similar to the results for triangular lattices in that for the heterogeneous site strength case more sites are required to break before the patterns diverge. This set of trials is different from those discussed previously in that $r .00$ and r.04 are the most similar. Thus for this case more perturbation is required to cause the crack patterns to diverge.

\subsubsection{Summary and Discussion of Perturbed Square Lattice}

\section{Single Trials}

Summary data for the perturbed square trials in given in Table 5.7-1. This table presents percolation thresholds and peak stresses for the homogeneous and heterogeneous site strength trials discussed above, and shows that for these trials increasing the range of the perturbation radius caused a decrease in the percolation threshold for the homogeneous site strength trials, but did not have a major effect on the heterogeneous site strength trials. Moreover, the homogeneous site strength trials had lower percolation thresholds as expected. This is consistent with the results from the single trials on the perturbed triangular lattice. The peak strength values for the homogeneous trials were all very similar, while the increase in lattice 


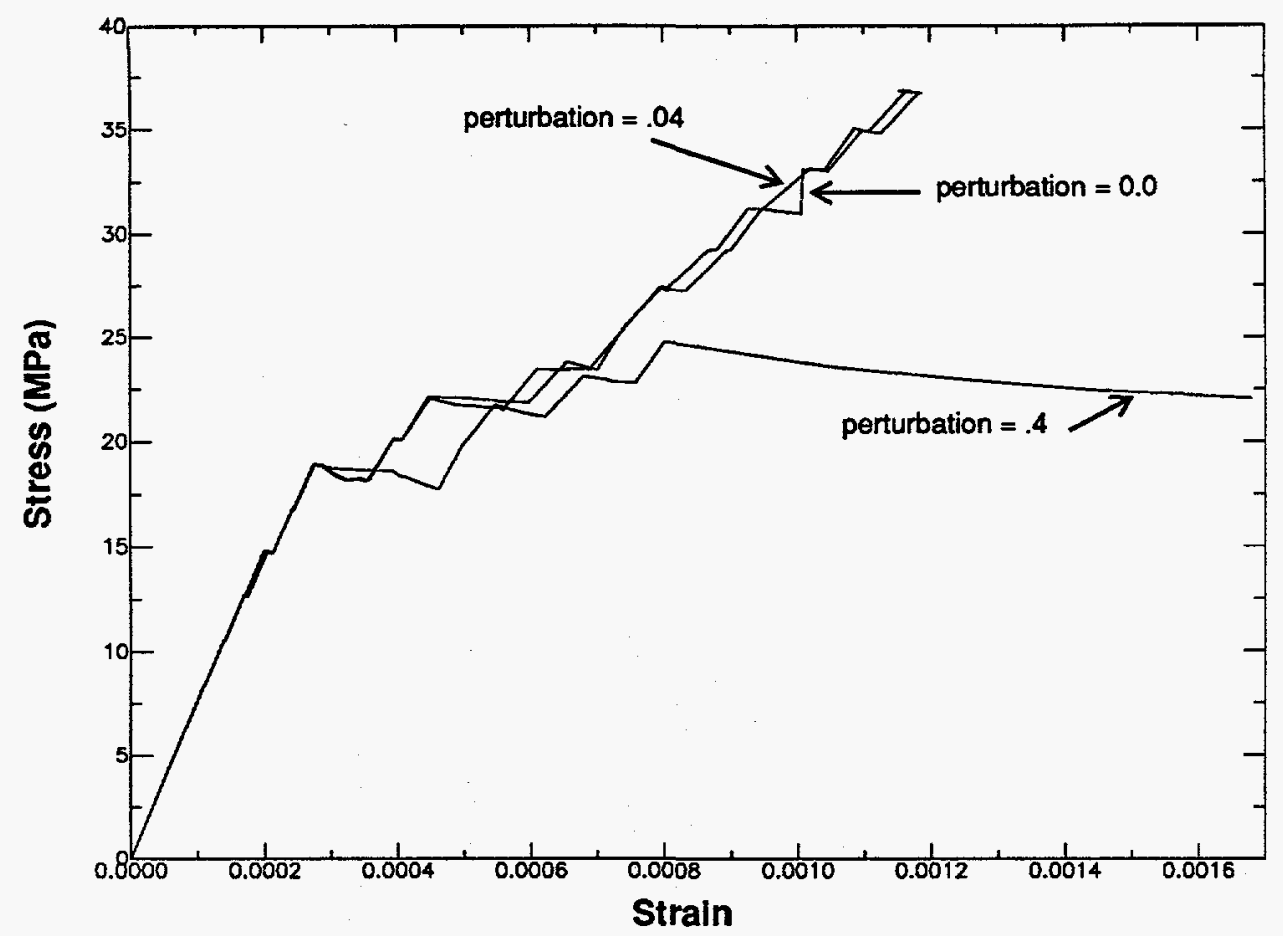

Figure 5.7-5 Effect of perturbation of a square lattice on stress strain curves for trials computed using a heterogeneous site strength distribution.

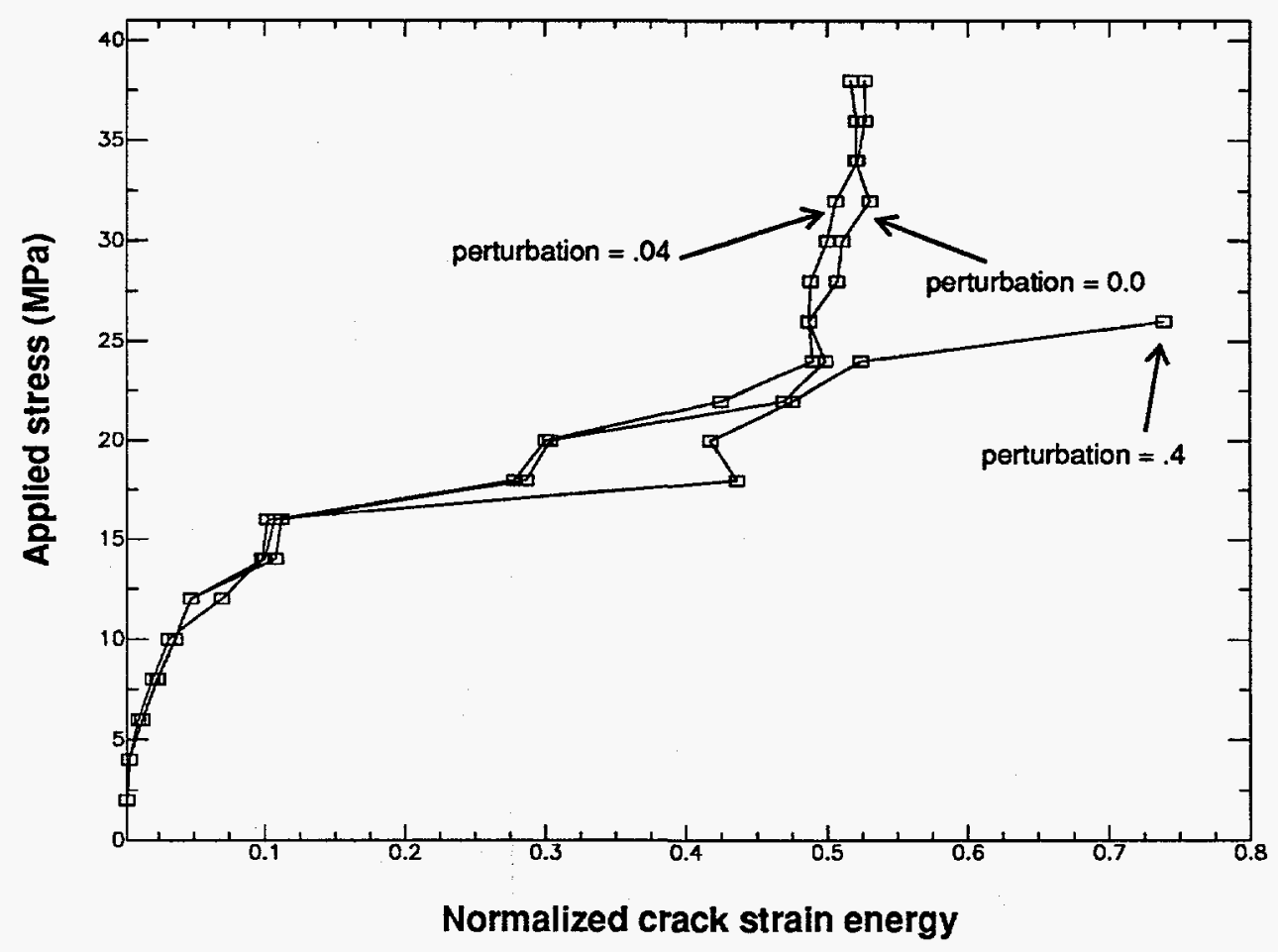

Figure 5.7 - 6 Effect of perturbation of a square lattice on relation of applied stress to normalized crack strain energy for trials computed using a heterogeneous site strength distribution. 


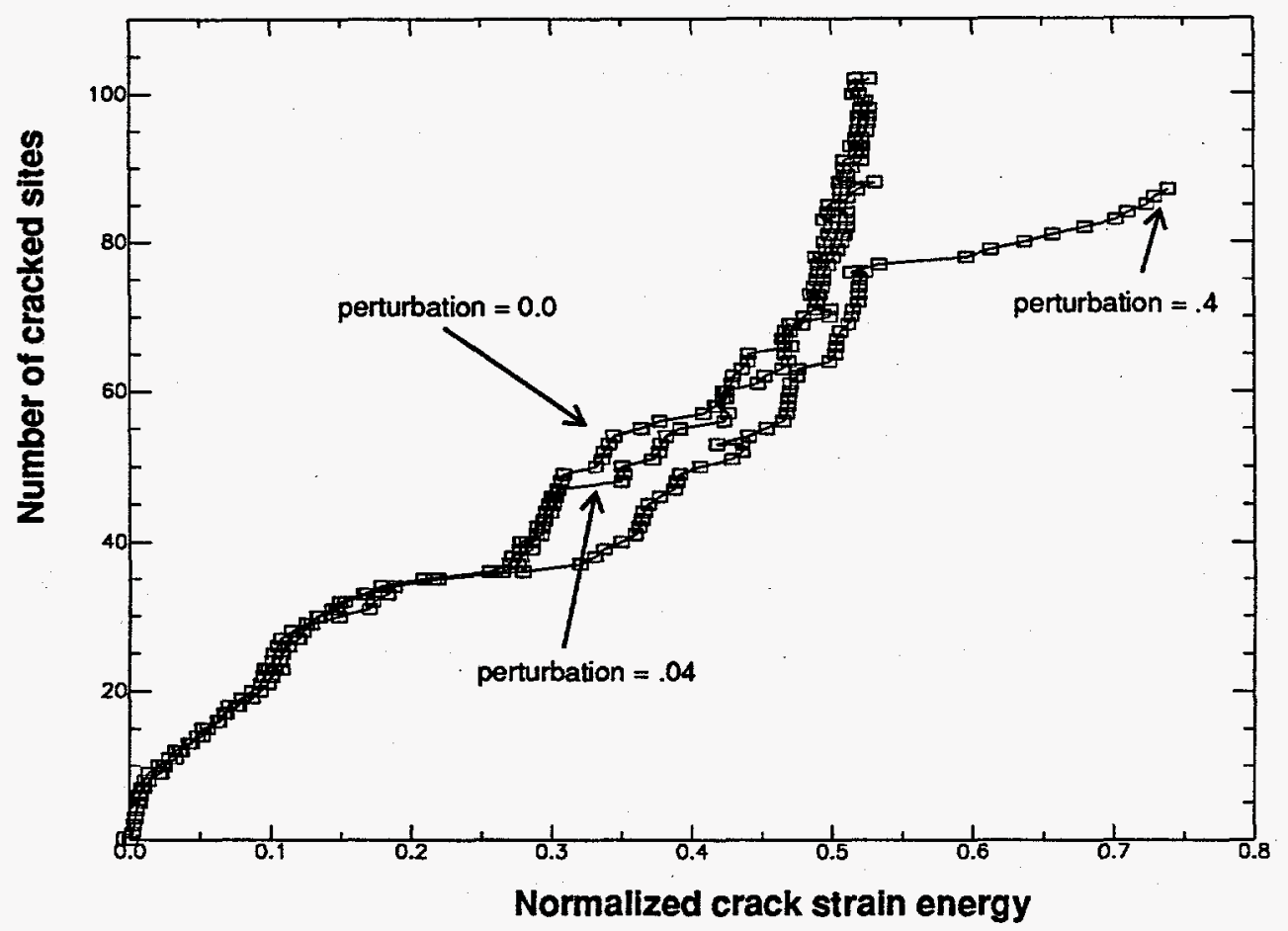

Figure 5.7-7 Effect of perturbation of a square lattice on relation of number of cracked sites to normalized crack strain energy for trials computed using a heterogeneous site strength distribution. 

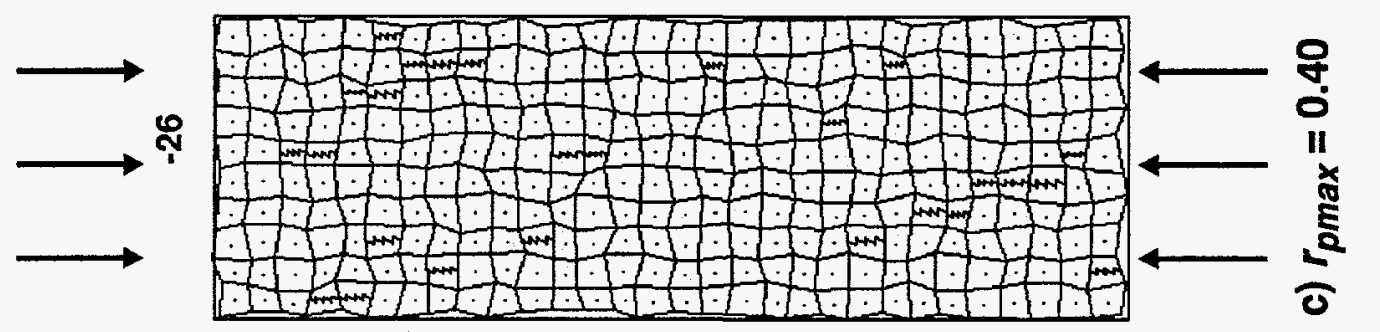

른
$\frac{1}{0}$
0
$\frac{0}{0}$
$\frac{0}{0}$
0
0

둥

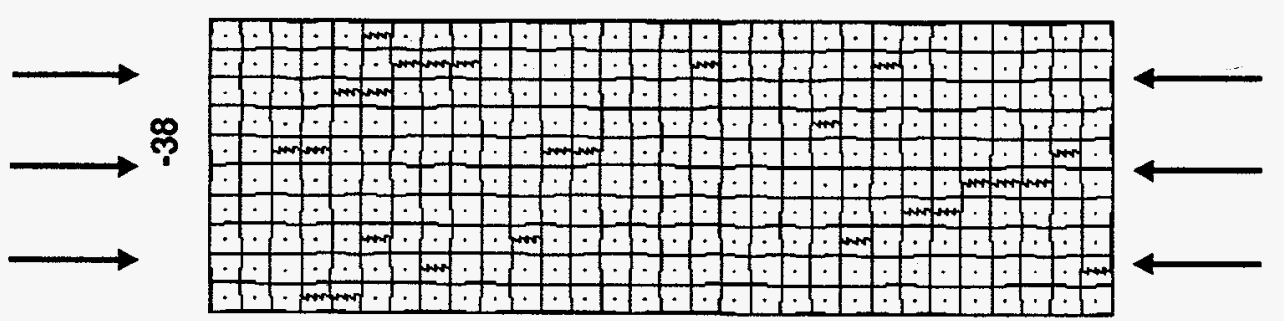

85

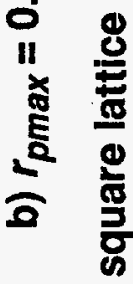

4

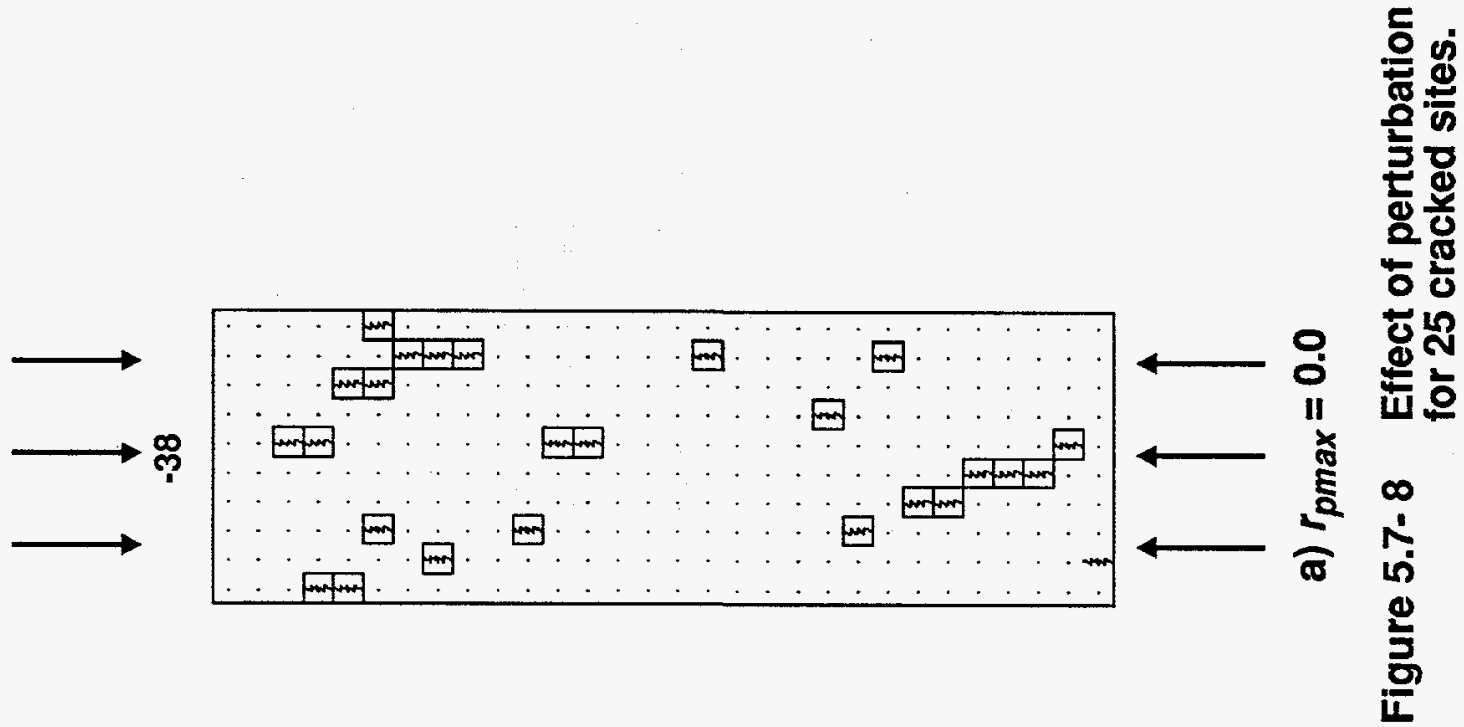



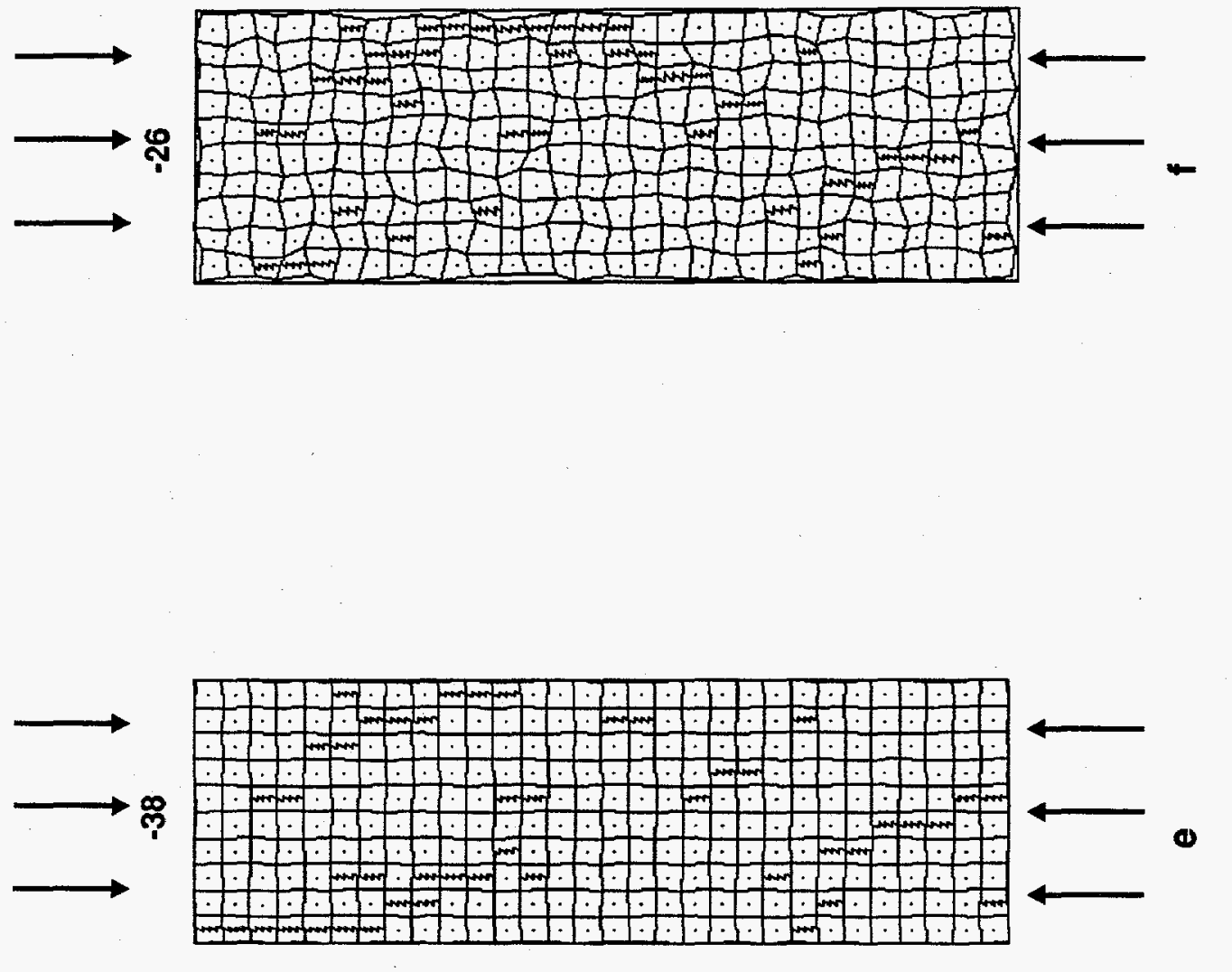

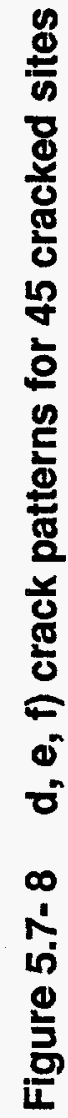

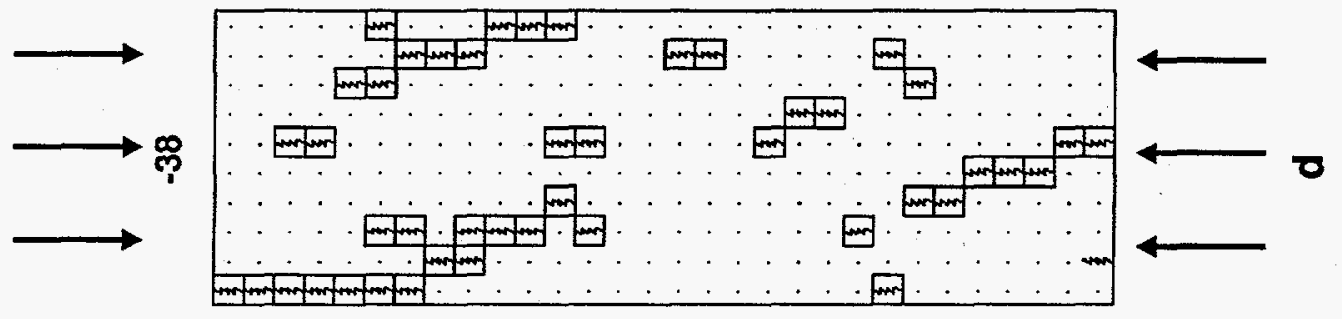



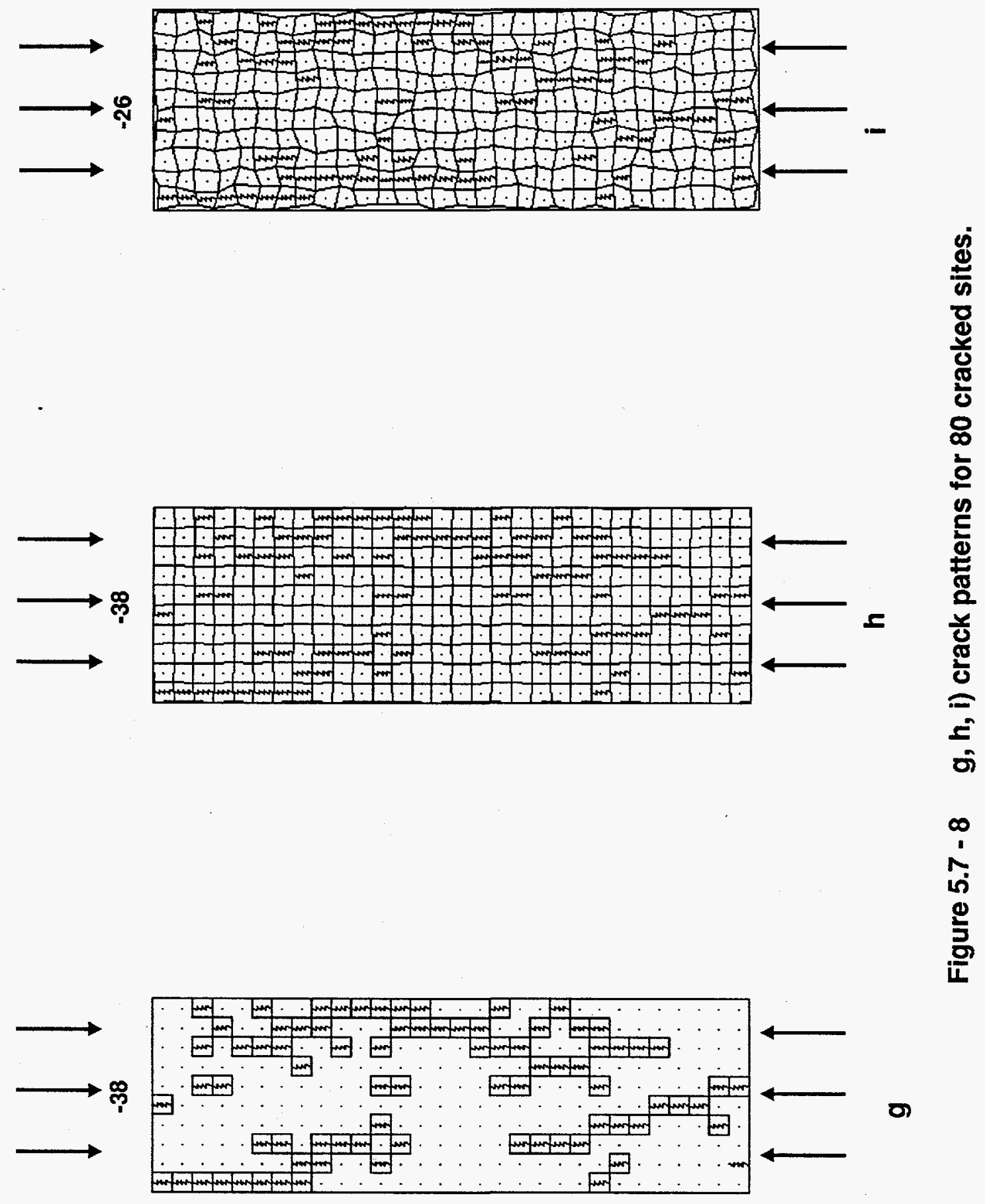

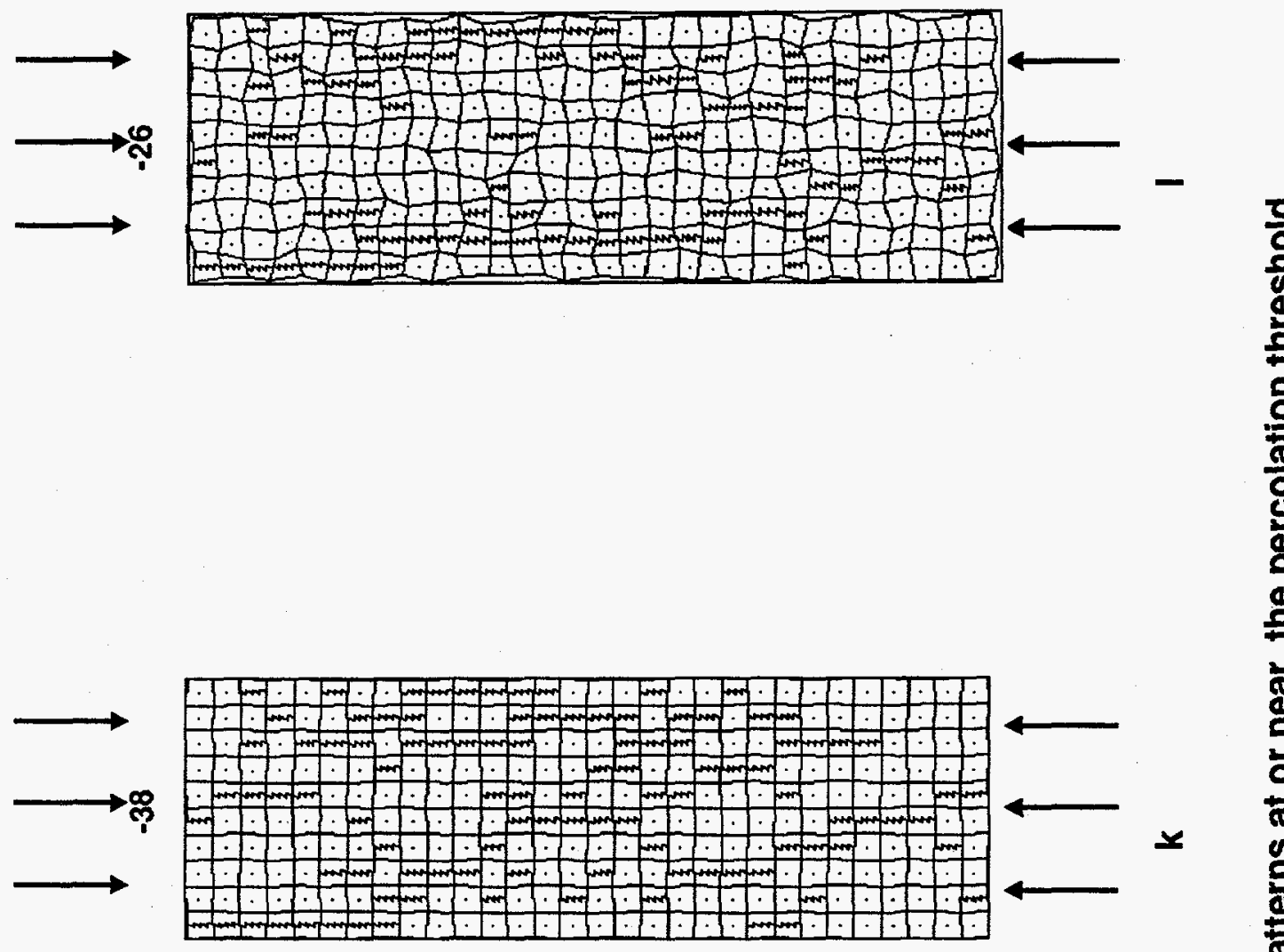

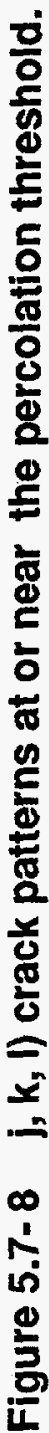

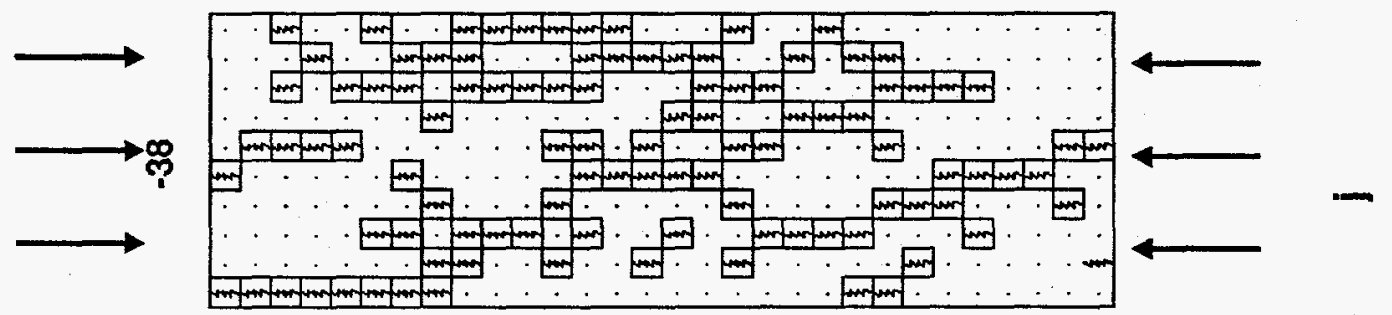


Table 5.7-1. Data for single trials for perturbed square lattice.

\begin{tabular}{ccccccccccccc}
\hline & & \multicolumn{1}{c}{ Init. } & \multicolumn{3}{c}{$\sigma_{\text {pk }}$} \\
Date & Lat. $^{\mathrm{a}}$ & JSZ & ISZ & $r_{\mathrm{p}_{\max }}$ & $\sigma_{\mathrm{I}}$ & $\Delta \sigma_{\mathrm{I}}$ & $T_{g}$ & $m_{1}$ & $\mathrm{~V}^{\mathrm{b}}$ & seq & (MPa) & $p_{\mathcal{C}}$ \\
\hline 28-Jun-94 & psqr & 10 & 30 & 0 & 2 & 2 & $20 \pm 0.2$ & .7 & 35.7 & 0 & 32.5 & 0.23 \\
28-Jun-94 & psqr & 10 & 30 & 0.04 & 2 & 2 & $20 \pm 0.2$ & .7 & 35.7 & 0 & 32.0 & 0.14 \\
28-Jun-94 & psqr & 10 & 30 & 0.40 & 2 & 2 & $20 \pm 0.2$ & .7 & 35.7 & 0 & 31.7 & 0.16 \\
& & & & & & & & & & & & \\
27-Jun-94 & psqr & 10 & 30 & 0 & 2 & 2 & $20 \pm 19.9$ & .7 & 35.7 & 6 & 36.7 & 0.34 \\
27-Jun-94 & psqr & 10 & 30 & 0.04 & 2 & 2 & $20 \pm 19.9$ & .7 & 35.7 & 6 & 36.8 & 0.34 \\
27-Jun-94 & psqr & 10 & 30 & 0.40 & 2 & 2 & $20 \pm 19.9$ & .7 & 35.7 & 6 & 24.8 & 0.29 \\
\hline
\end{tabular}

${ }^{a}$ Lattice type. ${ }^{b}$ Version of program.

perturbation caused a decrease in peak strength for the heterogeneous site strength trials.

These results are preliminary and reflect only one set of trials; more extensive study is required to verify the findings.

\section{Multiple Trials}

In addition to the controlled trials discussed above, sets of 10 trials were also computed. Ultimate strengths and percolation thresholds for these trials were very similar to those computed to the unperturbed square lattice (Table 5.7-2). This brief analysis indicates that slight perturbation of the square lattice has very little effect on the macroscopic strength.

Table 5.7-2. Mean values of ultimate strength and percolation threshold for perturbed square lattice. $m_{1}$ in \pm 0.7 for all trials.

\begin{tabular}{clccc}
\hline Trials & Site strength & $\begin{array}{c}\text { Perturbation } \\
\text { radius }\end{array}$ & $\sigma_{\text {pk }}$ & $p_{\mathrm{c}}$ \\
\hline 2_17_94 & $20 \pm .2$ & $\leq 0.20$ & 32.1 & $0.17 \pm 0.04$ \\
2_17_94 & $20 \pm 19.9$ & $\leq 0.20$ & $31.3 \pm 6.4$ & $0.26 \pm 0.05$ \\
3_14_94 & $20 \pm .2$ & $\leq 0.40$ & $30.9 \pm 2.7$ & $0.19 \pm 0.05$ \\
3_14_94 & $20 \pm 19.9$ & $\leq 0.40$ & $28.8 \pm 5.1$ & $0.31 \pm 0.05$ \\
\hline
\end{tabular}


Perturbation of the square array differs from that for the triangular arrays as follows. For the triangular arrays it was mentioned earlier that perturbation can move a site into a zone of enhanced stress or a zone of reduced stress. For the square arrays perturbation of the site location almost always moves the site into a zone of reduced stress. This is because for the compressive experiments, the stress field is aligned with the lattice and when a crack is inserted parallel to the $\sigma_{\mathrm{I}}$ direction, the zone of increased tension ahead of the crack tip will have highest stress levels exactly along the axis direction. Thus the unperturbed points are located at precisely the right place to experience the highest available stress, and perturbing the location will almost always move the point to a position of lower stress.

\subsection{Summary and Conclusions of Parameter Study}

An extensive parameter-sensitivity analysis has been conducted that considered lattice size, heterogeneity in the local stress field and in site strength, and heterogeneity in site location. The analysis included both triangular and square lattices. Major conclusions are presented below.

(1) The field-theory model exhibits an implicit size effect that closely matches laboratory and field data for the dependence of sample strength on sample size. This is an inverse power law relation with exponent of 0.17 . No assumption of flaw size is needed to generate this effect, and the effect is associated with a decrease, with increasing size, in the stress level required to create crack-interaction. The cause of this effect may be that, due to the increased number of possible ways that cracks can interact in the larger system, a weak path is easier and/or more likely to form.

(2) The field-theory model also implicitly predicts the development of macroscopic fracture surfaces similar to those observed in experiments on rocks to be formed by shear localization. These fracture surfaces develop even though a simple crack element is used that does not include shear. This effect is thought to be caused by the local heterogeneity incorporated into the field-theory model. 
(3) Heterogeneity in local stress field (due to grain shape and loading) also has a firstorder effect on macroscopic properties. In particular: increasing local stress heterogeneity lowers mean ultimate strength following an inverse power law, and makes the predicted stress-strain behavior more realistic.

(4) Perturbation of lattice sites caused development of en-echelon patterns of cracked sites that step across the array from the lower left to the upper right. Moreover, differences in crack patterns for perturbed and unperturbed trials were observed immediately (after as few as four cracked sites). Increasing heterogeneity in the lattice site locations decreased crack localization and decreased normalized crack strain energy. This is consistent with the postulate that systems with increasing disorder require more energy to break. During the initial phase of site cracking (less than $10 \%$ of the sites cracked), the perturbed lattices displayed progressively less strain energy at a given number of cracked sites. Moreover, for the perturbed lattices, at the percolation threshold a larger proportion of the total strain energy was due to cracked sites.

(5) Heterogeneity in site strength distribution had a relatively minor effect on macroscopic behavior. Peak strength is dependent on the mean site strength and not the width of the site strength distributions. Stress-strain behavior is affected by heterogeneity in site strength, with increasing amounts of strength heterogeneity causing more nonlinearity in the stress-strain curves and more strain-softening behavior.

\section{Discussion}

This study revealed that percolation thresholds are much lower than those predicted from stochastic models, and consequently statistical models for rock fracture need to consider alternative percolation algorithms such as directed-bond percolation, as the standard percolation models may not be appropriate for analysis of systems where crack interaction dominates behavior at a low fraction of sites broken. This is in agreement with the analysis of Bebbington (1992). Statistical models also need to emphasize heterogeneity in the local stress field, rather than site strength as a mechanism for introducing grain scale heterogeneity. 
One important aspect of material heterogeneity that was not addressed in this study is the existence of spatial correlation in the material properties. This is a fertile area for study, as it includes investigation of how macroscopic anisotropy in material properties is dependent on spatial correlation present in the microstructure, and incorporation of spatially correlated parameters such as layering at a variety of scales.

At the microstructural scale the concept of spatial correlation of microscale properties could be used to simulate strong grain centers surrounded by weaker grain boundaries. Moreover, using spatial correlation of microscale properties, the model can easily be configured to study the effect of heterogeneity at a variety of length scales as well as layered structures on macroscopic behavior. To do this, regions of higher or lower strength could be defined in the discretized region. 


\subsection{Analysis of Stress-Strain Behavior}

The goal of this chapter is to use the field-theory model to interrogate the macroscopic stress-strain curve for a simulated laboratory compression test in order to study the process of deformation and failure in a heterogeneous material. The approach is to use the model to show how the macroscopic stress-strain behavior is related to crack strain energy, the locations of cracked sites, and the formation, extension, and linking of clusters of cracked sites in simulations of laboratory compression tests. This was done by using output from the model to determine the number and location of cracked sites at any point on the stress-strain curve, and then displaying the normalized crack strain energy and overstress values associated with those cracks and the pattern of cracked sites. A stress-strain curve for a $30 \times 90$ simulation is analyzed in Section 6.1 and results are summarized in Section 6.2 and discussed in terms of a similar laboratory experiment. This analysis is relevant to problems in rock mechanics and rock engineering because it may provide the basis for techniques to recognize the onset of crack propagation and rock fracture. This would be useful in anticipating/preventing rock bursts, and for assessing mine pillar stability and the stability of boreholes and other underground openings. Techniques for recognition of fracture nucleation and propagation could also be applied to earthquake prediction.

\subsection{Interrogation of Macroscopic Stress-Strain Behavior}

Figure 6.1-1 presents a stress-strain curve computed for a 30×90 array with site strength distribution $T_{g} 20 \pm 19.9$, and $m_{1}$ in the range \pm 0.7 . These conditions were chosen to resemble properties of a clean, well-sorted sandstone such as Berea sandstone. This stress-strain curve shows linear elastic behavior up to a stress of approximately $15 \mathrm{MPa}$, then shows some strain softening, followed by an increase in stress to a peak of nearly $17 \mathrm{MPa}$, again followed by strain softening. The stress-strain curve has been divided into five intervals for the purpose of discussion, and each interval is discussed in the paragraphs below. Applied stress is plotted 
vs. normalized crack strain energy in Figure 6.1-2. This figure shows that at the formation of the percolating cluster, most of the strain energy computed for this trial was due to cracked sites. Moreover, most of the strain energy was gained at an applied stress of $16 \mathrm{MPa}$. This plot has also been divided into five intervals to provide cross reference to the intervals of stressstrain denoted in Figure 6.1-1. Figure 6.1-3 plots the number of cracks Vs normalized crack strain energy for this trial and shows that approximately 480 sites cracked in this trial (percolation threshold of approximately 0.18 ). This curve has also been divided into five intervals that correspond to the intervals denoted in Figure 6.1-1.

\section{Interval $a$}

Interval $a$ of the stress-strain curve shows linear response up to an axial stress of approximately $12 \mathrm{MPa}$. This is illustrated in Figure 6.1-2, which shows that for this interval very little crack strain energy was gained as applied stress was increased, and the increase in crack strain energy was linearly proportional to the level of applied stress. This indicates that most of the strain energy computed for this interval was due to continuum deformation of the system. This is also reflected in Figure 6.1-3, which shows that the normalized crack strain energy increased slowly and linearly with the 162 sites that were cracked over several levels of stress. It is also useful to examine the overstress plot of cracks formed in this interval. Figure 6.1-4 shows values of overstress plotted vs. the number of sites broken for applied stress levels in this interval (2-12 MPa). This figure shows that for an applied stress of $2 \mathrm{MPa}$, overstress generally decreased as the number of cracked sites increased. This type of behavior has been shown by de Arcangelis (1990) and interpreted to indicate that heterogeneity in site strength dominates the cracking of sites. At stress levels from 4 to $12 \mathrm{MPa}$, overstress vs. number broken is essentially constant at values between 1 and $2 \mathrm{MPa}$. The locations of sites cracked in interval $a$ are shown in Figure 6.1-5 a. This figure shows that the cracked sites associated with this interval are distributed randomly throughout the lattice and include a few small clusters of 5 to 10 cracks each. One small cluster, marked A, is noted for future reference. The linear increase in normalized crack strain energy over multiple stress levels with an increasing number 
of cracked sites and the random nature of the locations of the cracked sites, and the constant low level of the overstress curve, indicate that these cracked sites represent a population of noninteracting cracks in the array. Thus, the linear response of the stress-strain curve over interval $a$ corresponds to the cracking of sites at random locations and the formation of a population of non-interacting cracks in a material. This also indicates that constant or slightly decreasing values of overstress in the range of 1 to 2 are also indicative of random cracking of sites. Further, the decreasing values of overstress vs. number of cracked sites are consistent with site strength dominating site cracking. Constant values of overstress may imply that heterogeneity in both site strength and site geometry dominates site cracking.

\section{Interval $b$}

Interval $b$ of the stress-strain curve (Figure 6.1-1) shows stress-strain response as applied stress is raised to and held at $14 \mathrm{MPa}$. The stress-strain behavior at this applied stress is strain softening and is accompanied by an increase in the amount of strain energy associated with cracks, as is evidenced by the change in the slope between intervals $a$ and $b$ in Figure 6.1-2. Figure 6.1-3 shows that for interval $b$, the slope of the curve for number of cracks vs. strain energy is consistent with the slope of the sites cracked in interval $a$ until a total of approximately 175 sites are cracked. Then the slope of the curve changes as more crack strain energy is accumulated per cracked site. Overstress values for sites cracked in this interval are shown in Figure 6.1-6 and show a similar trend. This plot shows that the overstress values stayed nearly constant with values near 1.1 for the first 14 sites cracked in this interval. This is similar to the behavior shown for interval $a$. Locations of the sites cracked in this interval are highlighted in Figure 6.1-5 b, and are plotted along with the cracked sites from interval $a$. This figure shows that these 14 sites are randomly located, in much the same manner as those sites cracked in interval $a$, and that cluster A remains unchanged while a small cluster has formed at B. Figure 6.1-6 also shows that for the remaining sites cracked in interval $b$, the overstress values are erratically distributed in the general range 1 to 3 , with one site having a value much higher. The location of the sites cracked during this erratic overstress behavior is 
shown in Figure 6.1-5 bb, which shows that these cracked sites extend cluster B to form a major cluster along the left central portion of the array that is linear and aligned parallel to the maximum principal stress. Note that cluster A remains unchanged during this interval. Closer examination shows that most of the strain and gain in crack strain energy over this sub-interval is associated with cracked sites 175 to 206 , after which a few randomly located sites were broken. Thus the strain softening observed in interval $b$ of the stress-strain curve is due to the extension of cluster $B$, and is associated with a fundamental change in the behavior of the overstress plot and a change in slope of the normalized crack strain energy curve. The fundamental change in the overstress plot indicates that crack interaction dominates the site cracking process, and not heterogeneity in site strength and/or site geometry. This also shows that crack interaction can dominate behavior when less than $10 \%$ of the sites are cracked. Moreover, the cluster stops extending when 212 sites are cracked, and the model stops producing cracked sites at this stress level. The extension of cluster B may be similar to stable crack growth, and is interpreted to represent a fundamental change in behavior in the system from the random pattern of site cracking found for interval $a$, and the first part of interval $b$, to a regime where crack interaction influences behavior.

\section{Interval $c$}

The stress-strain response in interval $c$ in Figure 6.1-1 represents the initial behavior when the applied stress was raised to $16 \mathrm{MPa}$ after site cracking had stopped at the applied stress of $14 \mathrm{MPa}$ (interval $b$ ). Stress-strain values for this interval show a peak in stress followed by strain softening. The change in normalized crack strain energy over interval $c$ is not reflected in Figure 6.1-2. This is because this figure only shows the total normalized crack strain energy accumulated at a given level of applied stress, and only a portion of the total crack strain energy accumulated at the applied stress of $16 \mathrm{MPa}$ is due to cracks that form during interval $c$. Figure 6.1-3 shows that in this interval, crack strain energy per crack is greater than in interval $b$, and that approximately 65 additional sites cracked in this interval (cracked sites 214 to 270). The locations of these cracked sites are highlighted in Figure 6.1-5 $\mathrm{c}$ and are shown along 
with the locations of cracked sites for intervals $a$ and $b$. This figure shows that the sites cracked in this interval extended the large cluster B downward a distance approximately equal to its original length, making this cluster the dominant feature of the simulation, while cluster A remains unchanged. The overstress values computed for sites cracked in interval $c$ are plotted in Figure 6.1-7. These values show erratic behavior with values ranging between 1.1 and 8.0, similar to that observed for the cluster extension phase of interval $b$. Thus, the behavior during interval $c$ is that of strain softening associated with crack extension and erratic overstress values. This is similar to results for the latter portion of interval $b$, except that the crack interaction forces appear to be stronger in interval $c$, as is evidenced by the increased slope of the curve in Figure 6.1-3 and the larger range of overstress values. This is interpreted to mean that crack interaction dominated the behavior in this interval.

\section{Interval $d$}

Interval $d$ of the stress-strain curve in Figure 6.1-1 shows increased strain softening from that exhibited in intervals $b$ and $c$. Moreover, Figure 6.1-3 shows that between 55 and 60 sites were cracked during this interval, but the overall change in crack strain energy was small (note that the change in normalized crack strain energy over interval $d$ is not reflected in Figure 6.1-2 for the same reasons given for interval $c$ ). Sites cracked in interval $d$ are highlighted in Figure 6.1-5 d along with sites cracked from the previous intervals. Most of these new cracked sites are randomly located in the upper and lower quarters of the array, and a new cluster, C, has formed above cluster A while clusters A and B are essentially unchanged. Cracks in this interval also formed small extensions to some of the branches of the major cluster A. Moreover, overstress values for this interval shown in Figure 6.1-7 show a marked change in from those computed for interval $c$. The values computed for sites cracked in this interval are generally in the range 1 to 2 and are more consistent with the constant values observed for interval $a$ than with the erratic values observed for interval $c$ (and part of $b$ ). Figure 6.1-3 shows that for many of the sites cracked in this interval, crack strain energy remained unchanged. Further examination shows that the small increases in crack strain energy are associated with 
formation of the cluster $C$. The scattered distribution of cracked sites and the relatively constant values of overstress and crack strain energy are similar to the behavior observed in interval $a$, except that for interval $d$, strain softening is predicted. The reduced stress level associated with strain softening results from the transfer of stress to side columns as sites are cracked. However, as mentioned above, 55 sites are cracked during this interval $(d)$, and these produced approximately one-half of the strain produced by the 66 sites cracked in interval $c$. Thus, even though strain softening is predicted, the behavior is more characteristic of the regimes where site scale heterogeneity dominates the cracking of sites. This may be analogous to crack arrest and damage formation and/or the formation of a damage zone ahead of a crack or fracture in rock and rocklike materials.

\section{Interval $e$}

This sub-section discusses interval $e$ of the stress-strain curve shown in Figure 6.1-1. Behavior in this interval shows continued strain softening with the same slope as interval $d$, except that the strain in interval $e$ is due to only a few cracked sites. Moreover, Figure 6.1-3 shows a significant increase in crack strain energy with the cracking of each site in the interval. In fact, sites cracked in this interval contribute approximately $13 \%$ of the total normalized crack strain energy for this trial. The locations of these cracked sites are shown in Figure 6.1-5e, which shows that these are sites that link the main cluster, B, with smaller clusters ( $C$ and others) and extend B nearly to the top of the array. These are defined as critical connecting sites. The overstress values computed for these cracked sites are plotted in Figure 6.1-7 and show that generally the overstress values increase steadily over the interval. This behavior represents a regime where crack interaction controls the cracking of sites. This regime is similar to the crack interaction regimes observed for intervals $b$ and $c$, except that in this interval major clusters are linked and much larger gains in strain and strain energy occur. This regime may be associated with unstable crack growth. 


\subsection{Summary and Discussion}

The analysis presented in Section 6.1 shows that the field-theory model predicts three main regimes of behavior in simulations of laboratory compression tests on rock. In the first regime, heterogeneity in site strength and/or site geometry determines the number and location of cracked sites at a given level of applied stress. In this regime, the cracked sites are randomly located and generally do not interact. In the second regime, crack interaction controls the location of sites that crack at stress level. In this regime, clusters of cracked sites form, extend, and coalesce. In the third regime critical connecting crack are formed that link clusters. This regime may be associated with catastrophic failure. Results also indicate that fracture under compressive loading a typical sequence of fracture development may occur as follows.

(1) Formation of initial damage (regime 1): Upon axial loading, a population of randomly located, non-interacting sites are cracked. Some small clusters of cracked sites may form and extend or coalesce, but they have no significant effect on the macroscopic stress-strain behavior, and do not contribute a significant amount of energy to damage material. The stress-strain behavior is nearly elastic during this stage. In this stage site scale heterogeneity dominates the fracture process.

(2) Emergence of crack interaction (regime 2): When 10 to $15 \%$ of the sites are cracked some small clusters of cracked sites extend or coalesce, forming a few larger clusters. Strain softening occurs and crack strain energy increases dramatically when the clusters link or extend.

(3) Crack arrest and formation of additional damage (regime 1): This is an interval when more non-interacting sites are cracked. This may be similar to the formation of a process zone ahead of a crack. Stress-strain behavior shows strain softening, with very little change in strain or strain softening for each crack.

(4) Formation of critical connecting cracks (regime 3): During this stage the larger clusters extend, often by linking with smaller clusters formed during steps 1 or 3. Strain softening 
behavior is produced. Macroscopic strain and crack strain energy show very large increases when a site cracks, and especially when a few critical connecting sites are cracked.

At any given level of stress all regimes may occur. The analysis indicates that the regimes described by de Arcangelis (1990) also are useful for describing the development of fracture observed in this study.

Comparison of these results with the experimental results of Lockner et al. (1991) shows that the model correctly predicts the different regimes of crack nucleation and propagation that have been observed in compressive tests on granite samples, where the axial load was servo-controlled to keep the rate of acoustic emission constant. These investigators found as axial load was raised, the linear elastic portion of the stress-strain curve was associated with acoustic emissions that were randomly located throughout the sample. Non linear strainhardening occurred prior to the peak stress and then post-peak strain-softening was also observed. The strain-softening was associated with the nucleation and propagation of a planar zone of acoustic emissions inclined to the axis of the sample. This is generally the same progression of cracking that is shown in Figures 6.1-4 a-e.

Moreover, Lockner et al. observe variability in the energy release rate computed for the experiment, and attribute this to heterogeneity in strength in the sample. Results reported in Chapter 5 indicate that this may be due to heterogeneity in grain shape and loading geometry and not the local strength of grains or cementing agents. The results presented here also indicate that the stepping of the cracks may be due to the microscale heterogeneity in the rock.

One difficulty in using acoustic emission to monitor fracture is that it is not clear what physical occurrence an acoustic emission actually represents (Bebbington et al. 1990). The fieldtheory model could be used to study this problem by employing clustering algorithms designed to simulate acoustic emissions with different energy levels. Results could then be compared with the experimental data to evaluate different algorithms and identify those that correctly reflect the observed acoustic emissions and associated parameters. 
The evolution of a fracture can also be studied by examining the clusters of cracked grains as the density of cracked grains $p$ approaches $p_{c}$. If $s$ is defined as the cluster size (number of cracks in a cluster), and $n_{s}$ as the number of clusters of size $s$, then at $p_{c}$ the relation between $n_{s}$ and $s$ has the form:

$$
n_{s}\left(p_{c}\right) \sim s^{-\tau}
$$

where $\tau$ is the Fisher exponent (Stauffer, 1985). This is a general relation and it has been shown that $\tau$ has the same value for several different two-dimensional lattices (Stauffer, 1985). Moreover, $\tau$ is one of a small family of exponents that are postulated to depend only on the dimensionally of a lattice and not on the type of lattice (e.g., square or triangular) nor on the type of percolation model used. This property is called universality and for a given problem allows a lattice to be selected that is appropriate to that problem. Other measures that can be used as $p$ approaches $p_{c}$ are cluster radius (or radius of gyration) and the connectedness correlation function

$$
G(r)=\frac{e^{-r / \xi}}{r^{d-2+\eta}}
$$

where $r$ is the distance between points, $d$ is the lattice dimension, $\eta$ is another critical exponent, and $\xi$ is the correlation length, which diverges with a critical exponent $v$ near $p_{c}$ as

$$
\xi \sim\left|p-p_{c}\right|^{-v}
$$

Evaluation of these expressions using data produced using the model will provide an indication of their usefulness for describing rock fracture, especially if an appropriate clustering algorithm is used. 


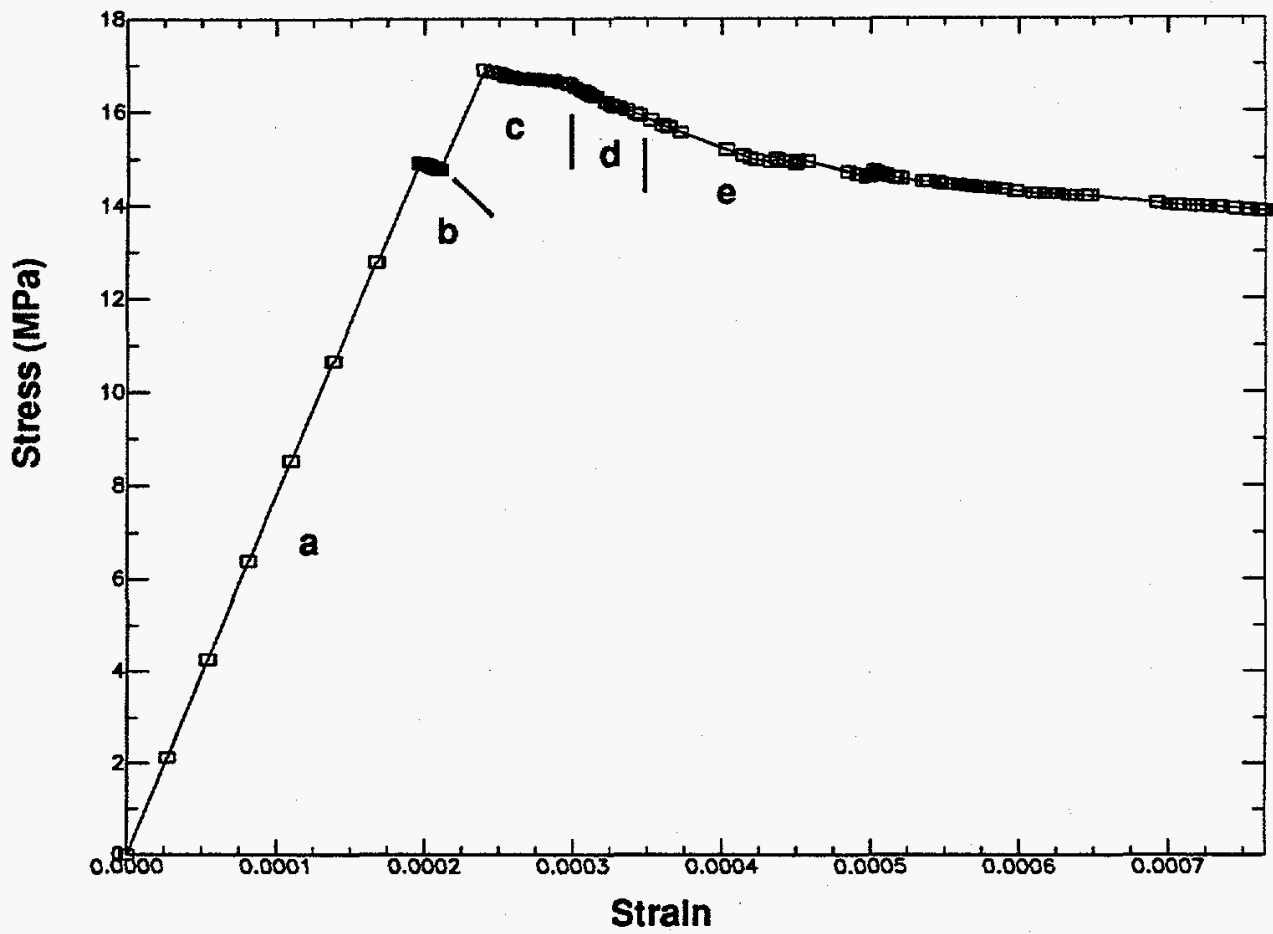

Figure 6.1 - 1 Stress strain curve for $30 \times 90$ array.

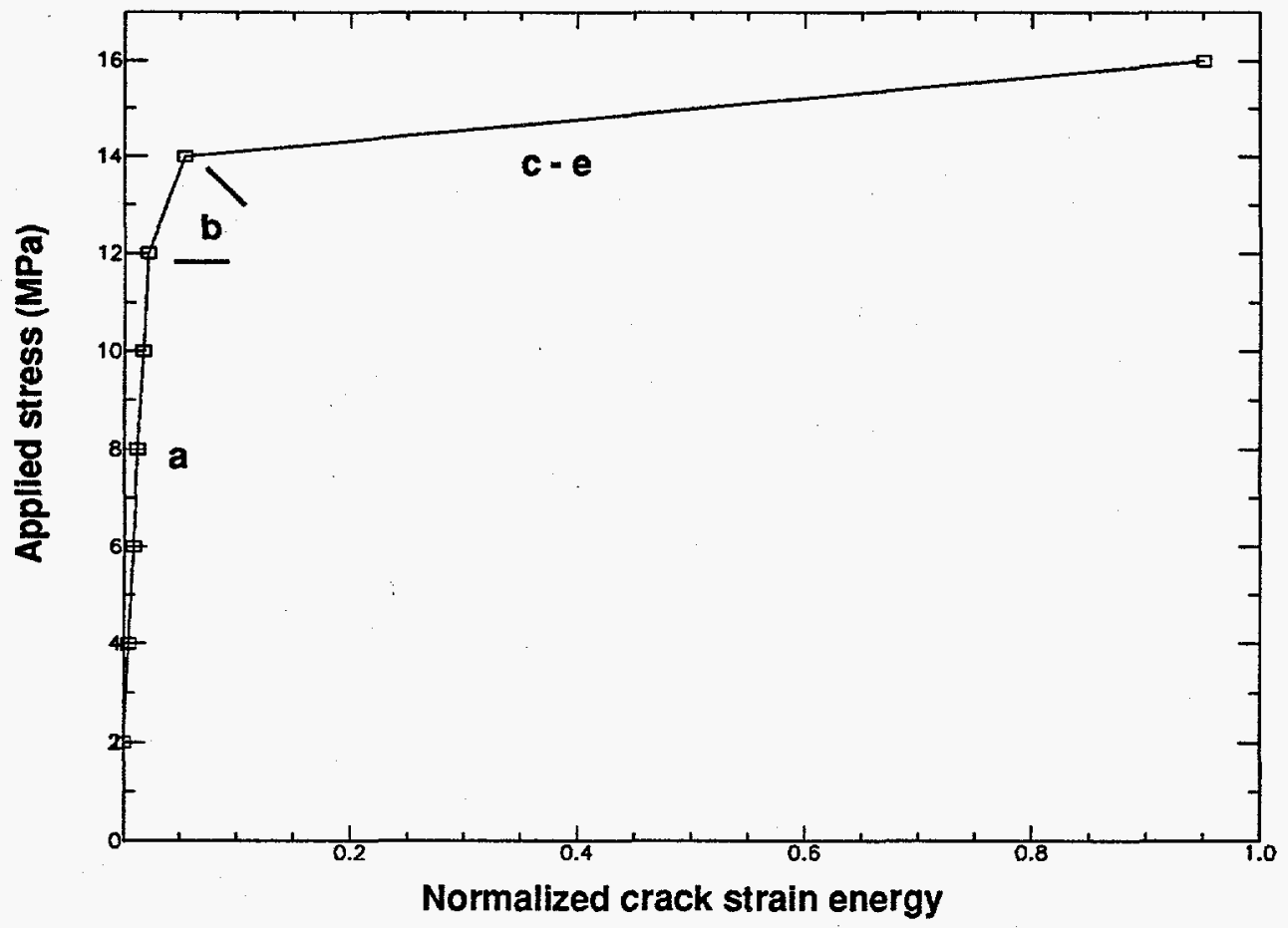

Figure 6.1 - 2 Relation of applied stress to normalized crack strain energy for $30 \times 90$ array. 


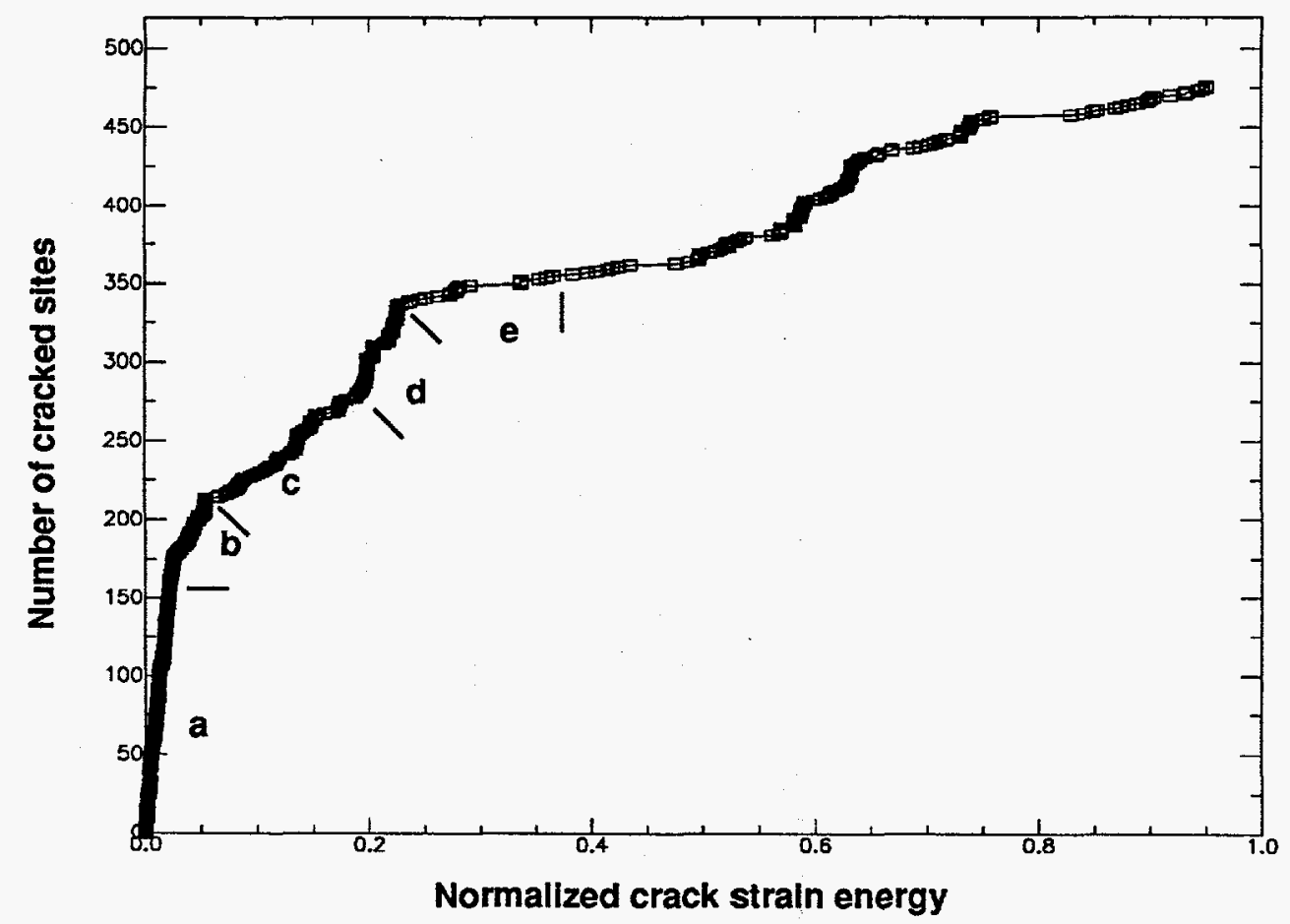

Figure 6.1 - 3 Relation of number of cracked sites to normalized crack strain energy for $30 \times 90$ array.

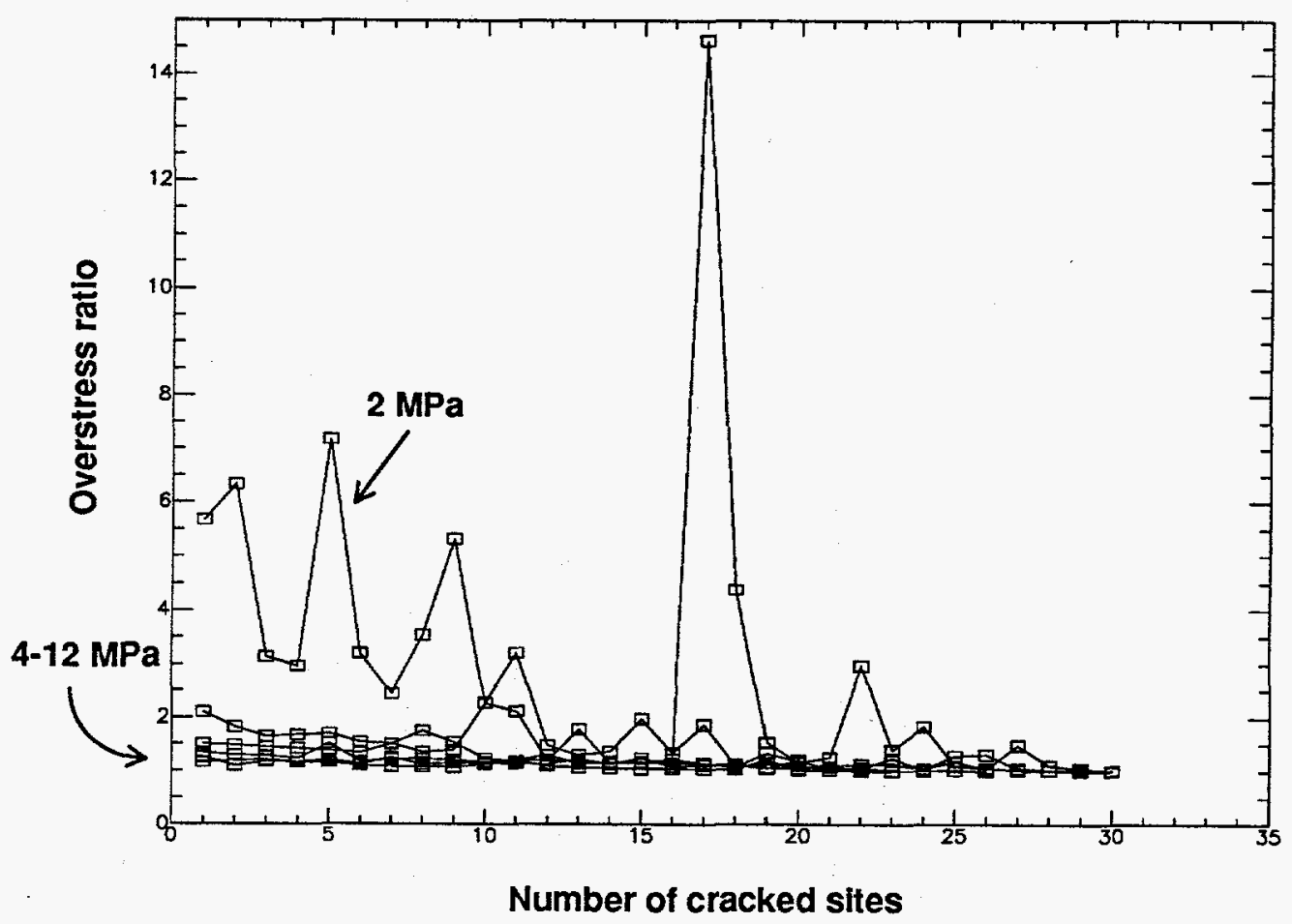

Figure 6.1-4 Relation of overstress ratio to number of cracked sites at a given stress level for $30 \times 90$ array. 

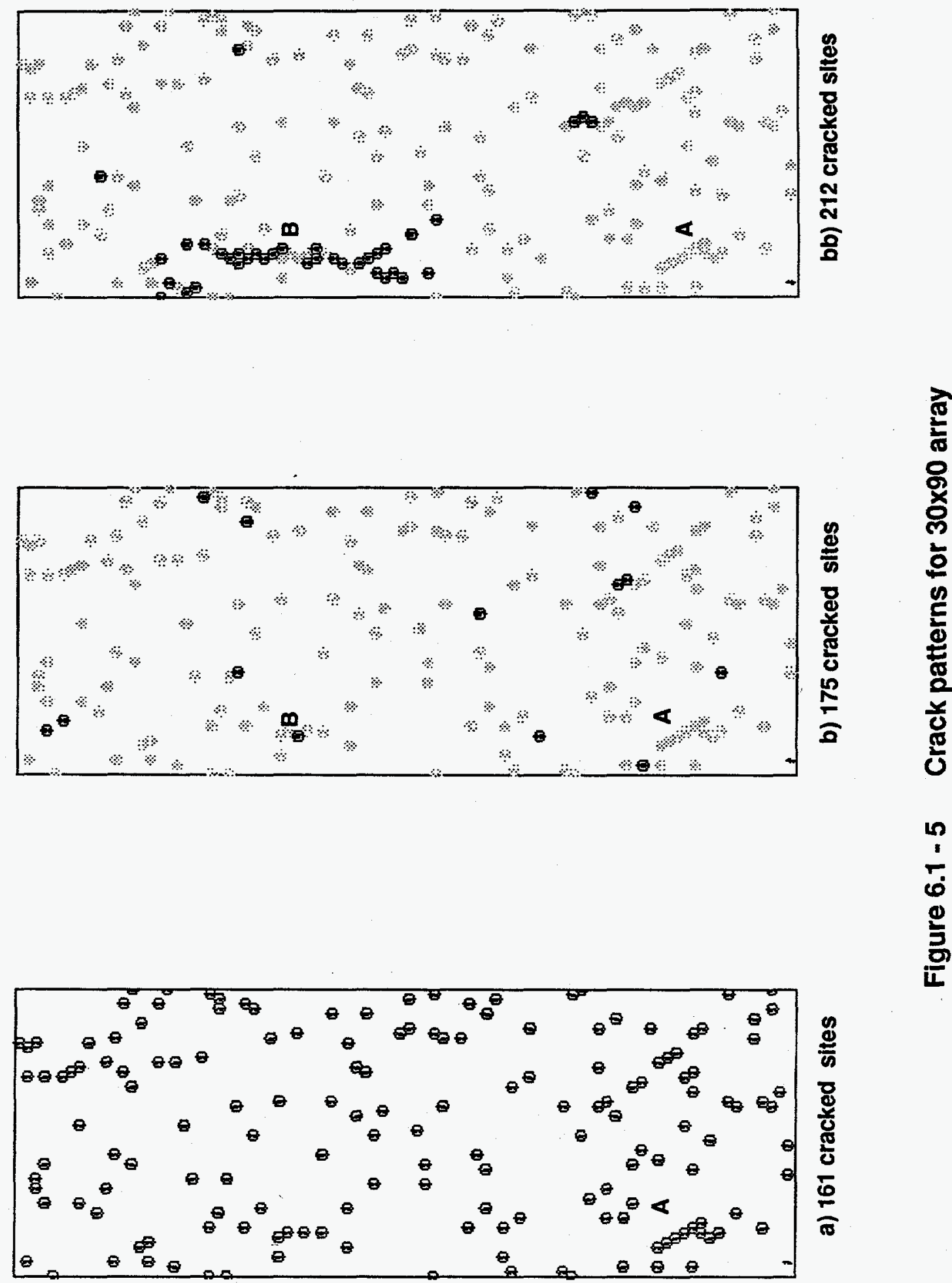

n
1
0
0
$\frac{9}{3}$
$\frac{1}{4}$ 

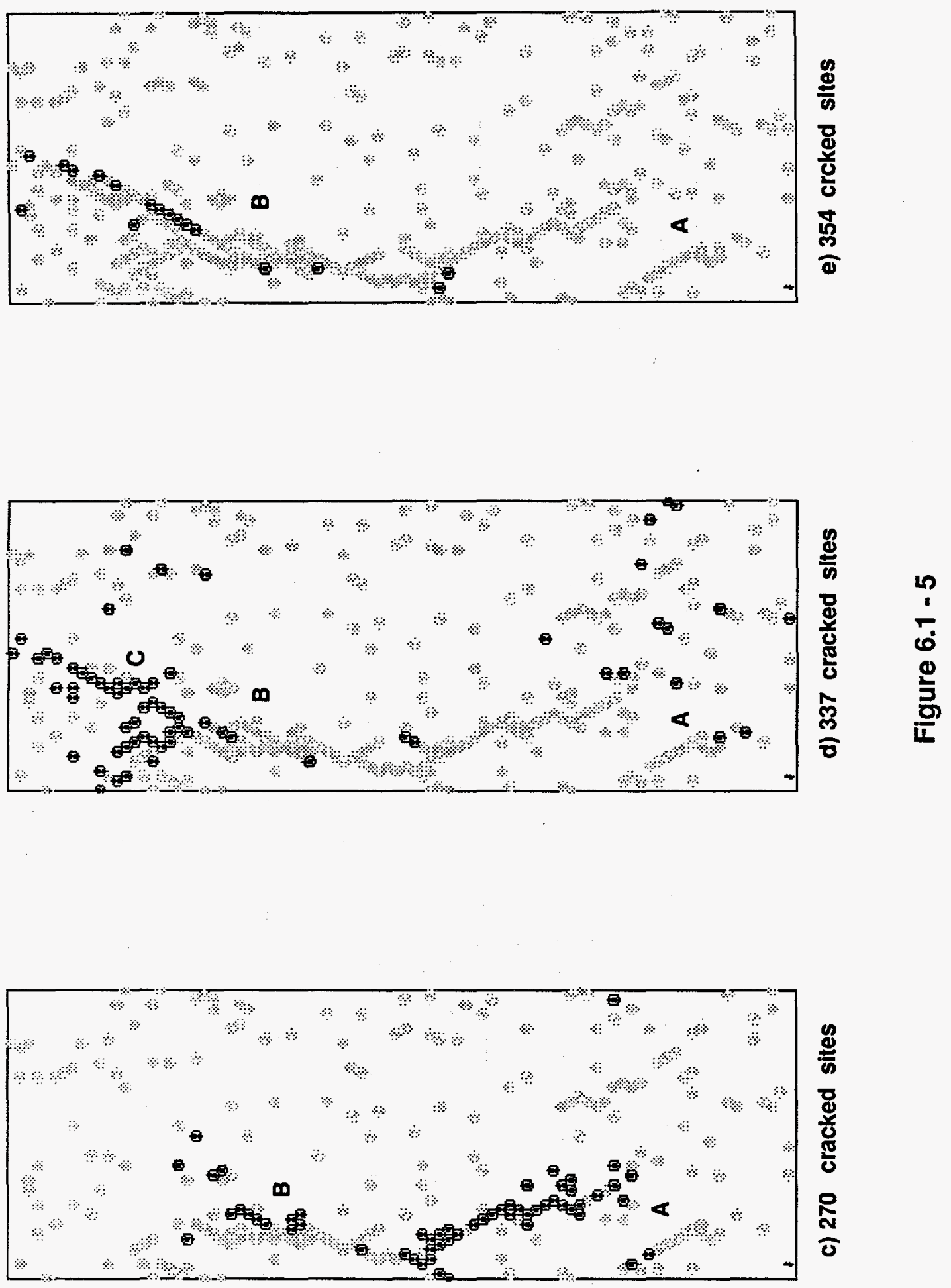


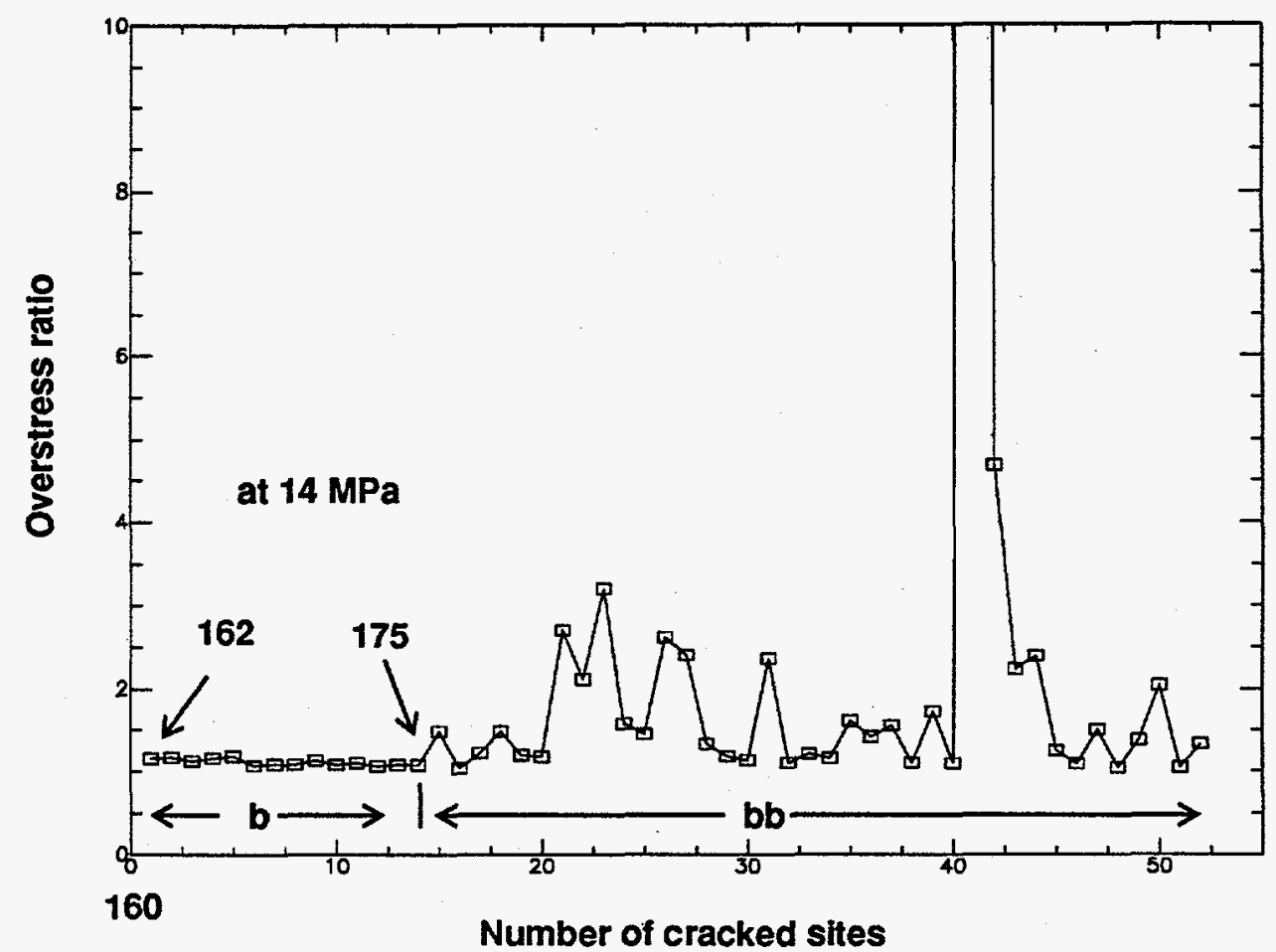

Figure 6.1 - 6 Relation of overstress ratio to number of cracked sites at an applied stress of $14 \mathrm{MPa}$.

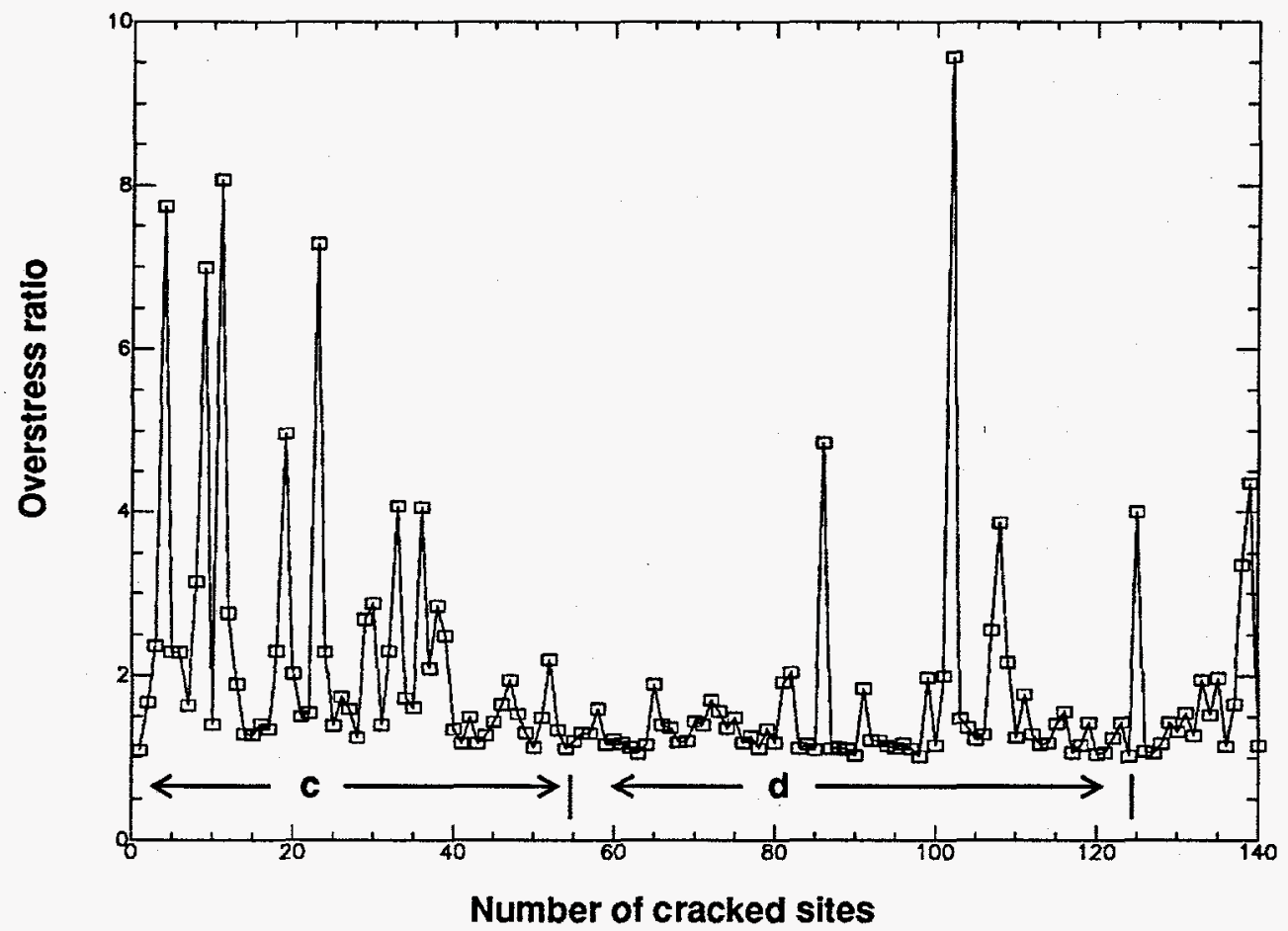

Figure 6.1-7 Relation of overstress ratio to number of cracked sites for intervals $c$ and $d$ of stress strain curve for $30 \times 90$ array. 


\subsection{Conclusions}

This dissertation has presented two different numerical models for rock deformation and fracture in compression. The models are called the field-theory and nearest-neighbor models and were developed to provide a method for study of the effect of microscale heterogeneity on macroscopic properties and fracture of rock. These models incorporate a few fundamental assumptions based on laboratory and analytical results developed over the last 30 years. These models simulate grain breaking, and provide for formation of rock damage and rock fracture via the formation, extension, and coalescence of grain-scale cracks.

This dissertation also presents the results of a parameter-sensitivity study and a detailed analysis of stress-strain behavior performed using the field-theory model. Input parameters used in this modeling study were based on experimental and field observations and on analytical solutions of idealized geometries. Scale factors were not used to fit the output to any selected data set.

\section{Major Results}

The field-theory model implicitly predicts fracture patterns and size dependence similar to those observed in tests on rock.

Results show that the field-theory model implicitly predicts the development of shear-type fractures even though a simple crack element that does not include shear stress is used. This implies that the en-echelon nature of cracking observed in rocks in compression may be due to the local heterogeneity in the rock, and that the local heterogeneity leads to crack interactions along macroscopic surfaces that are similar to those caused by shear localization and that are often observed in real experiments.

The model also exhibits an implicit size effect that closely matches laboratory and field data for the dependence of sample strength on sample size. This is an inverse power-law relation with exponent similar to that obtained in analysis of laboratory and field data on rock strength. No 
assumption of flaw size is needed to generate this effect, and the effect is associated with a decrease with increasing size in the stress level required to create crack interaction. This may be because the larger population of sites offers more possible ways for cracked sites to interact and a larger number of ways for a weak path to be formed.

\section{Heterogeneity in local stress is found to be an important parameter.}

It was found that heterogeneity in the local stress field, such as that due to variations in the shape and loading of grains, has a first-order effect on the macroscopic mechanical properties predicted for simulations of uniaxial compression tests. In particular: increasing heterogeneity in the local stress field lowers mean ultimate strength following an inverse power law. Analysis of the results indicates that this may be because changing the amount of heterogeneity in local stress perturbation changes how energy is partitioned; increasing local stress heterogeneity causes a larger proportion of the available energy to go into crack formation, and causes crack interaction to become dominant at lower levels of applied stress.

It was also determined that the amount of heterogeneity in the local stress field affects the shape of the stress-strain curves, and that increasing the amount of heterogeneity lowered the modulus and introduced more strain-softening. This is because more cracks are allowed to form at lower stresses (reducing the modulus) and more energy goes into crack formation, and crack interaction occurs at lower stresses. Implications for real materials are that increased heterogeneity in the local stress field actually homogenizes the behavior, as it causes cracks to arrest and then restart, and more sites are available for cracking, making it easier to start another crack. This result is also consistent with the observation that small amounts of heterogeneity in grain shape are important in pure materials.

This study also revealed that the amount of heterogeneity in the local stress field due to grain shape and loading present in most rocks is in the range where this parameter has a large effect on ultimate strength. Moreover, the inverse dependence of ultimate strength on local stress heterogeneity means that larger amounts of heterogeneity have less effect. It is important to note 
that the range of values used for the stress difference multiplier were based on analytical solutions for specific geometries observed in sandstone, and that increasing local stress heterogeneity had no significant effect on percolation threshold.

\section{Interrogation of a typical stress-strain curve shows three regimes of behavior.}

The field-theory model was found to predict three main regimes of behavior in simulations of laboratory compression tests on rock. In the first regime heterogeneity in site strength and/or local stress field determines the number and location of the cracked sites, and these sites are randomly located and generally do not interact. In this regime, stress-strain behavior is generally linear elastic. In the second regime, crack interaction controls the location of sites that crack. In this regime, clusters of cracked sites form and extend, producing stress-strain behavior that is generally strain-softening. Moreover, this work indicates that crack interaction can become important when as few as $10 \%$ of the sites are cracked. In the third regime, major clusters are connected by the formation of a few critical connecting cracks. This regime may be equivalent to unstable crack extension, with major crack extension and release of energy occurring when only a few sites crack. Results of this analysis also indicate how these regimes may be used to characterize rock fracture under compressive loading:

(a) Formation of initial damage. Regime 1: between 10 and $20 \%$ of the sites are cracked; linear elastic stress-strain behavior.

(b) Clusters of cracked sites form and extend. Regime 2: the emergence of crack interaction; strain-softening.

(c) Crack arrest and formation of additional damage. Return to Regime 1: strainhardening or minor strain-softening.

(d) Rock fracture. Regime 3: critical connecting cracks form to unite major clusters. 
This study provides evidence that crack interaction becomes important when a very low fraction $(10 \%)$ of the sites are cracked, and this produces low percolation thresholds. Moreover, this effect is enhanced as array size is increased.

\section{Lattice perturbation causes stepping of crack locations.}

This study revealed that stepping of cracks observed in laboratory tests and the field may be due to the heterogeneity in grain packing. This is based on results for perturbed and unperturbed lattices that show that perturbing the lattice caused a change in crack patterns, with the crack patterns for perturbed trials showing crack stepping. For homogeneous site strength, perturbing the lattice geometry affects crack locations immediately, after as few as four sites are cracked, while for the heterogeneous site strength distribution, approximately $15 \%$ of the sites are cracked before perturbation of the lattice geometry begins to affect location of cracked sites. This may indicate the crack density at which geometry begins to dominate site strength in the cracking process. Increasing heterogeneity in the lattice site locations decreased crack localization and decreased normalized crack strain energy. This is consistent with the postulate that systems with increasing disorder require more energy to break. During the initial phase of cracking (less than $10 \%$ of the sites cracked), the perturbed lattices displayed progressively less strain energy at a given number of cracks. Moreover, for the perturbed lattices, at the percolation threshold a larger proportion of the total strain energy was due to cracks. The peak strength values for the homogeneous trials were all very similar, while the increase in lattice perturbation caused an increase in peak strength for the heterogeneous site strength trials (triangular).

\section{Other Observations}

It was found that the mean site strength is important in determining the macroscopic peak strength. Results show that for triangular lattices, heterogeneous site strength distributions yield ultimate strengths very similar to those for the homogeneous site strength trials at a variety of conditions where both site strength distributions were computed. Results for square lattices 
show that increasing heterogeneity in site strength lowered the peak strength and increased the percolation threshold by minor amounts. This is in contrast to the results for the triangular lattice, which showed no change in peak strength with increasing site strength heterogeneity.

This may be because on the square lattice, stronger crack interaction occurs.

Crack patterns were more realistic for heterogeneous site strength distributions, and percolation thresholds were higher, but peak strength was unaffected. This is important for the statistical modeling community, as many models introduce heterogeneity by using a stochastic distribution of breaking thresholds and they may not be of major importance for simulation of macroscopic properties of materials.

The values of the ratio $\mathrm{E} / \sigma_{\mathrm{pk}}$ (Young's modulus to ultimate strength) for many of the trials discussed in this dissertation are in the range $1000-3000$. This is considerably higher than values commonly associated with rocks, which are in the range 200-800 (Goodman, 1980). Moreover, in this study the model has not been used as a simulator to predict ultimate strength values; the purpose of the study is to learn how the microscale heterogeneity affects the behavior, and to examine trends in this behavior. The observed trends, such as the increase in ultimate strength as heterogeneity in the local stress distribution $\left(m_{1}\right)$ is decreased, are valid over a wide range of parameter values. Moreover, values of 300 are obtained for the $\mathrm{E} / \sigma_{\mathrm{pk}}$ ratio when a mean site strength of $50 \mathrm{MPa}$ and $m_{1}$ values in the range \pm 0.3 are used. This is consistent with the observation that $m_{1}$ values of 0.5 and 0.7 represent ideal grain geometries that are not commonly found in nature and suggests that a smaller range for the $m_{1}$ parameter may be more appropriate for simulation of rocks. It is also important to note that input parameters used in this modeling study were based on observations from laboratory and field experiments, and on analytical solutions of idealized two-dimensional geometries. Scale factors were not used to fit the output to any selected data set.

Finally, this study revealed that because crack interaction dominates behavior at a low fraction of sites broken, statistical models for rock fracture need to consider alternative percolation algorithms such as directed-bond or invasion percolation. This is in agreement with 
the analysis of Bebbington et al. (1992), who indicate that these models are better suited to simulation of rock fracture than are standard percolation algorithms. Statistical models also need to emphasize heterogeneity in the local stress field, rather than in site strength, as a mechanism for introducing grain-scale heterogeneity. 


\subsection{References}

American Society for Testing and Materials, Standard Test Method for Uniaxial Compression Tests, D2938-86, Philadelphia, PA (no date listed).

Ambegaokar, V., B. I. Halperin, and J. S,. Langer (1971), Phys. Rev. B 4, 2612.

Ashby, M. F., and S. D. Hallam (1986), "The failure of brittle solids containing small cracks under compressive stress," Acta Metall. 34, 497-510.

Bak, P., and K. Chen (1989), "The physics of fractals," Physica D 38, 5-12.

Bak, P., and K. Chen (1991), "Self-organized criticality," Sci. Am. 264(1), 46-53.

Barton, N., R. Lien, and J. Lunde (1974), “Engineering classification of rock masses for the design of tunnel support," Rock Mech. 6(4), 189-236.

Batzle, M. L., G. Simmons, and R. W. Siegried (1980), "Microcrack closure in rock under stress: direct observation," J Geophys. Res. 85, 7072.

Bebbington, M., D. Vere-Jones and X. Zheng (1990), "Percolation theory: a model for rock fracture?," Geophys. J. Int. 100, 215-220.

Becker, R. A., J. M. Chambers, and A. R. Wilks (1988), The New S Language (Wadsworth \& Brooks/Cole, Pacific Grove, CA).

Berryman, J. G., and S. C. Blair (1986), "Use of digital image analysis to estimate fluid permeability of porous materials: application of two-point correlation functions," J. Appl. Phys. 60, 6.

Bieniawski, Z. T. (1967), "Mechanism of brittle fracture of rock," Int. J. Rock Mech. Min. Sci. 4, 395430.

Bieniawski, Z. T. (1976), “Rock mass classifications in rock engineering," Proc. Symp. Explor. Rock Engineer., vol. 1 (Balkema, Rotterdam), 97-106.

Bieniawski, Z. T., and W. L. Van Heerden (1975), "The significance of in-situ tests on large rock specimens," Int. J. Rock. Mech. Min. Sci. 12, 101-113. 
Blair, S. C., and J. G. Berryman (1992), "Permeability and relative permeability of rocks," Fault Mechanics and Transport Properties of Rocks, Evans and Wong, eds. (Academic Press, London).

Blease, J. (1977), "Series expansions for the directed-bond percolation problem," J. Phys. C. Solid State Phys. 10.

Brace, W. F. (1971), "Micromechanics of rock systems, in structure, solid mechanics and engineering design," Proc. Southhampton 1969 Civil Eng. Mater. Conf., Part 1, M Te'Eni, ed. (Wiley-Interscience, New York), 187-204.

Brace, W. F. (1978), "Volume changes during fracture and frictional sliding: a review," Pure and Appl. Geophys. 116, 603-614.

Brace, W. F., B. Paulding, and C. H. Schultz (1966), "Dilatancy in the fracture of crystalline rock," J. Geophys. Res. 71, 3939-3954.

Brady, B. (1988), HEFF (Version 2.0) Users Guide, Itasca Consulting Group, report to U.S. Nuclear Regulatory Commission.

Bebbington, M., D. Vere-Jones, and X. Zheng (1990), "Percolation theory: a model for rock fracture," Geophys. J. Int. 100, 215-220.

Bruno, M. S., and R. B. Nelson (1991), "Microstructural analysis of the inelastic behavior of sedimentary rock," Mech. of Mater. 12, 95-118.

Chelidze , T. L., (1986), "Percolation theory as a tool for imitation for fracture process inrocks," PAGEOPH 124, 4/5.

Crawford, A. M., and J. H. Curran (1982), "Higher-order functional variation displacement discontinuity elements," Int. J. Rock Mech. Min. Sci. Geomech. Abstr. 19, 143-148.

Crouch, S. L., and A. M. Starfield (1990), Boundary Element Methods in Solid Mechanics (Unwin Hyman, Cambridge, MA).

Daveler, S. A., and S. C. Blair (1994), Fracrock Users' Guide, UCRL-MA-119099, Lawrence Livermore National Laboratory, Livermore, CA.

Davis, P. A. (1992), "Antiplane interacting cracks using the boundary element method on a Connection Machine," EOS 73, 43. 
de Arcangelis, L. (1990), "Randomness in breaking thresholds," Statistical Models for the Fracture Disordered Media, Hermann and Roux, eds. (North-Holland, Amsterdam).

Dey, T. N., and C. Y. Wang (1981), "Some mechanisms of microcrack growth and interaction in compressive rock failure," Int. J. Rock Mech. Min. Sci. 18, 199-209.

Domb, C., and M. S. Green (1972), Phase Transitions and Critical Phenomena, C. Domb and J. L. Lebowitz, eds. (Academic Press, London), 1-12.

Englman, R., and Z. Jaeger (1990), "Fracture in concrete due to percolating cracks and pores," Physica A.

Evans, I., and C. D. Pomeroy (1958), "The strength of cubes of coal in uniaxial compression," Mechanical Properties of Non-Metallic and Brittle Materials, W. H. Walton, ed. (Butterworths, London), pp. 5-28.

Evans, I., C. D. Pomeroy, and R. Berenbaum (1961), "The compressive strength of coal," Colliery Eng. 38, 75-80, 123-127, 172-178.

Ewy, R. T., and N. G. W. Cook (1990), "Deformation and fracture around cylindrical openings in rock-I. Observations and analysis of deformations," Int. J. Rock Mech. Min. Sci. 27, 387-407.

Fredrich, J. T., and T-f. Wong (1986), "Micromechanics of thermally induced cracking in three crustal rocks," J. Geophys. Res. 91, 12743-12764.

Germanovitch, L. N. (1993), University of Oklahoma, personal communication.

Gallagher, Jr., J. J., M. Friedman, J. Handin, and G. M. Sowers (1974), “Experimental studies relating to microfracture in sandstone," Tectonophysics 21, 203-247.

Goodman, R. E. (1980), Introduction to Rock Mechanics (John Wiley \& Sons, New York).

Hadley, K. (1976), "Comparison of calculated and observed crack densities and seismic velocities in Westerly granite," J. Geophys. Res. 81, 3484-3494.

Hallbauer, D. K., H. Wagner, and N. G. W. Cook (1973), "Some observations concerning the microscopic and mechanical behavior of quartzite specimens in stiff, triaxial compression tests," Int. J. Rock Mech. Min. Sci. 10, 713-726. 
Herrmann, H. J., and S. Roux (1990), Statistical Models for the Fracture of Disordered Media (NorthHolland Elsevier Science Publishers B. V.).

Hinrichsen, E., A. Hansen, and S. Roux (1989), Europhys Lett. 8, 1.

Hoek, E., and E. T. Brown (1980), Underground Excavation in Rock (The Institution of Mining and Metallurgy, London).

Holt, R. M., and E. Fjaer (1987), "Acoustic and mechanical behavior during failure," Expanded Abstracts, Soc. Expl. Geophys. 57th Annual Meeting, New Orleans, 14-18.

Horii, H., and S. Nemat-Nasser (1985), "Compression-induced microcrack growth in brittle solids: axial splitting and shear failure," J. Geophys. Res. 90, 3105-3125.

Horii, H., and S. Nemat-Nasser (1986), "Brittle failure in compression: splitting, faulting, and brittle-ductile transition," Phil. Trans. Royal Soc. London 319, 337-374.

Huang, J. I. (1992), "Modeling dynamic fracture growth with an elastic network," Geophys. Res. Lett. 19, 18.

Huang., J. I., and K. Kim (1993), “Fracture process aone development during hydraulic fracturing," Int. J. Rock Mech. Min. Sci. Geomech. Abstr.

Jaeger, J. C., and N. G. W. Cook (1976), Fundamentals of Rock Mechanics (Chapman and Hall, London).

Kapur, P. C. (1993), Dept. of Material Science and Mineral Engineering, Univ. of California at Berkeley, personal communication.

Kemeny, J. M., and N. G. W. Cook (1987), "Crack models for the failure of rock under compression," Proc. 2nd Int. Conf. Const. Laws for Eng. Mater. 2, 879-887.

Kemeny, J. M., and N. G. W. Cook (1991), "Micromechanics of deformation in rocks," Toughening Mechanisms in Quasi-Brittle Materials, S. P. Shaw, ed. (Klewer Academic, The Netherlands), 155-188.

Kesten, H. (1986), "Percolation theory and first passage percolation," Anal. of Probability 15 (4), 1231-1271.

Kesten, H. (1987), "Scaling relations for 2d-percolation," Common. Math. Phys. 109, 109-156. 
Kranz, R. L. (1979), "Crack growth and development during creep of Barre granite," Int. J. Rock Mech. Min. Sci. 16, 23-36.

Liu, Z., L. R. Myer, and N. G. W. Cook (1993), "Micromechanics of granular materials-numerical simulation of the effects of heterogeneities," Proc. 34th U.S. Rock Mech. Symp.

Lockner, D. A., J. D. Byerlee, V. Kuksenko, A. Ponomarev, and A. Sidorin (1991), "Quasi-static fault growth and shear fracture energy in granite," Nature 350, 39-42.

Lomnitz-Adler, J. (1985), "Asterity models and characteristic earthquakes," Geophys. J. R. A. S., 83.

Louis, E., F. Guinea, and F. Flores (1985), in Fractals in Physics, L. Pietronero and E. Tosatti, eds. (North Holland, Amsterdam).

Meakin, P. (1991), "Fractal aggregates in geophysics," Rev. Geophys. 29(3).

Meakin, P. (1992), “Models for material failure and deformation,” Science , 252.

Mora, P. (1992), "The atomic lattice solid model fracturing and stick-slip," EOS, Trans. Am. Geophys. Union 73, 43, supplement.

Myer, L. R., M. J. Kemeny, Z. Zheng, R. Suarez, R. T. Ewy, and N. G. W. Cook (1992), “Extensile cracking in porous rock under differential compressive stress," Appl. Mech. Rev. 45(8), 263380.

Olsson, W. A. (1974), "Microfracturing and faulting in a limestone," Tectonophysics 24, 277-285.

Peitgen, H.-O., and D. Sauge (1988), The Science of Fractal Images (Springer-Verlag, New York).

Peng, S. S., and A. M. Johnson (1972), "Crack growth and faulting in cylindrical specimens of Chelmsford granite," Int. J. Rock Mech. Min. Sci. 9, 37-86.

Price, R. H. (1986), Effects of Sample Size on the Mechanical Behavior of Topopah Spring Tuff, Sandia National Laboratories, Albuquerque, NM, SAND85-0709.

Renshaw, C. (1993), Stanford University, Palo Alto, CA, personal communication.

Ross, J., G. Narkounskaia, W. Klein, and J. B. Rundle (1992), “Dynamical properties of a cellular automaton model for earthquakes," EOS, Trans. Am. Geophys. Union 73, 43, supplement.

Rudnicki , J. W., and J. R. Rice (1975), J. Mech Phys. Solid 23, 371-395. 
Rundle, J. B., and W. Klein (1992), "Nonlinear dynamical models for earthquakes and frictional sliding: an overview," Proc. 33rd U.S. Symp. Rock Mech., J. R. Tillerson and W. R. Wawersik, eds., Sante Fe, NM, June 3-5, 1992.

Rundle, J. B., and W. Klein (1993), "Scaling and critical phenomena in a cellular auomaton slider block model for earthquakes," J. Stat. Physics 72,1/2.

Sahimi, M., and S. Arbabi (1992),"Percolation and fracture in disordered soldis and granular media: approach to a fixed point," Phys Rev. Lett. 68, 5.

Sammis, C. G., and M. F. Ashby (1986), "The failure of brittle porous solids under compressive stress states," Acta. Metall. 34, 511-526.

Schlangen, E. (1993), Experimental and Numerical Analysis of Fracture Processes in Concrete, Ph.D. Thesis, Technical University of Delft, The Netherlands.

Schlangen, E., and J. G. M. van Mier (1992), "Micromechanical analysis of fracture of concrete," Int. J. Damage Mechanic 1, 435-454.

Song, J. , and K. Kim (1994), "Numerical simulation of delay blasting by the dynamic lattice network model," Proc. 1st North American Rock Mech. Symp., Austin, Texas, June 1994 (Balkema).

Stauffer, D. (1985), Introduction to Percolation Theory (Taylor and Francis, London and Philadelphia).

Steacy, S. J., and C. G. Sammis (1991), "An automaton for fractal patterns of fragmentation," Nature 353(19), 250-252.

Suarez-Rivera, F. R., P. J. Cook, N. G. W. Cook, and L. R. Myer (1991), "The role of wetting fluids during the indentation of porous rocks," Rock Mechanics as a Multidisciplinary Science, Proc. 32nd U.S. Rock Mech. Symp., J. C. Rolgiers, ed. (Balkema, The Netherlands), 683-692.

Suarez-Rivera, F. R., Z. Zheng, N. G. W. Cook, and G. A. Cooper (1990), "Indentation by pore collapse in porous rocks," Proc. 31st U.S. Symp. Rock Mech., 671-678.

Tang, C. (1994), "Artificial and natural failure in rock testing," Int. Soc. Rock Mech. News J. 1, 4.

Wawersik, W. R., and W. F. Brace (1971), "Post-failure behavior of a granite and diabase," Rock Mech. 3, 61-85. 
Wawersik, W. R, and C. A. Fairhurst (1970), "A study of brittle rock failure in laboratory compression experiments," Int. J. Rock Mech. Min. Sci. Geomech. Abstr. 7, 561-575.

Wilkinson, D., and J. F. Willemsen (1983), "Invasion percolation: a new form of percolation theory," J. Phys. A Math Gen. 16, 3365-3376.

Wong, T.-f. (1982), "Micromechanics of faulting in Westerly granite," Int. J. Rock Mech. Min. Sci. 19, 49-64.

Zhang, J., T. F. Wong, and D. M. Davis (1990), "Micromechanics of pressure-induced grain crushing in porous rocks," J. Geophys. Res. 95, 341-352.

Zhao, Y., J. Huang, and R. Wang (1993), "Real-time SEM observations of the manufacturing process in rock during a compression test," Int. J. Rock Mech. Min. Sci. Geomech. Abstr. 30(6),

- 643-652.

Zheng, Z. (1989), Compressive Stress-Induced Microcracks in Rocks and Applications to Seismic Anisotropy and Borehole Stability, Ph.D. Thesis, University of California at Berkeley.

Zheng, Z., N. G. W. Cook, and F. M. Doyle (1987), "A new technique to observe threedimensional cracks in rocks," EOS, Trans. Am. Geophys. Union 68, 1477.

Zheng, Z., L. R. Myer, and N. G. W. Cook (1989), "Microcrack geometry in confined and unconfined conditions," Proc. 30th U.S. Symp. Rock Mech., 749-756. 
Appendix

Listing of Trials Computed for Parameter-Sensitivity Analysis 
Index of trials computed for parameter sensitivity analysis.

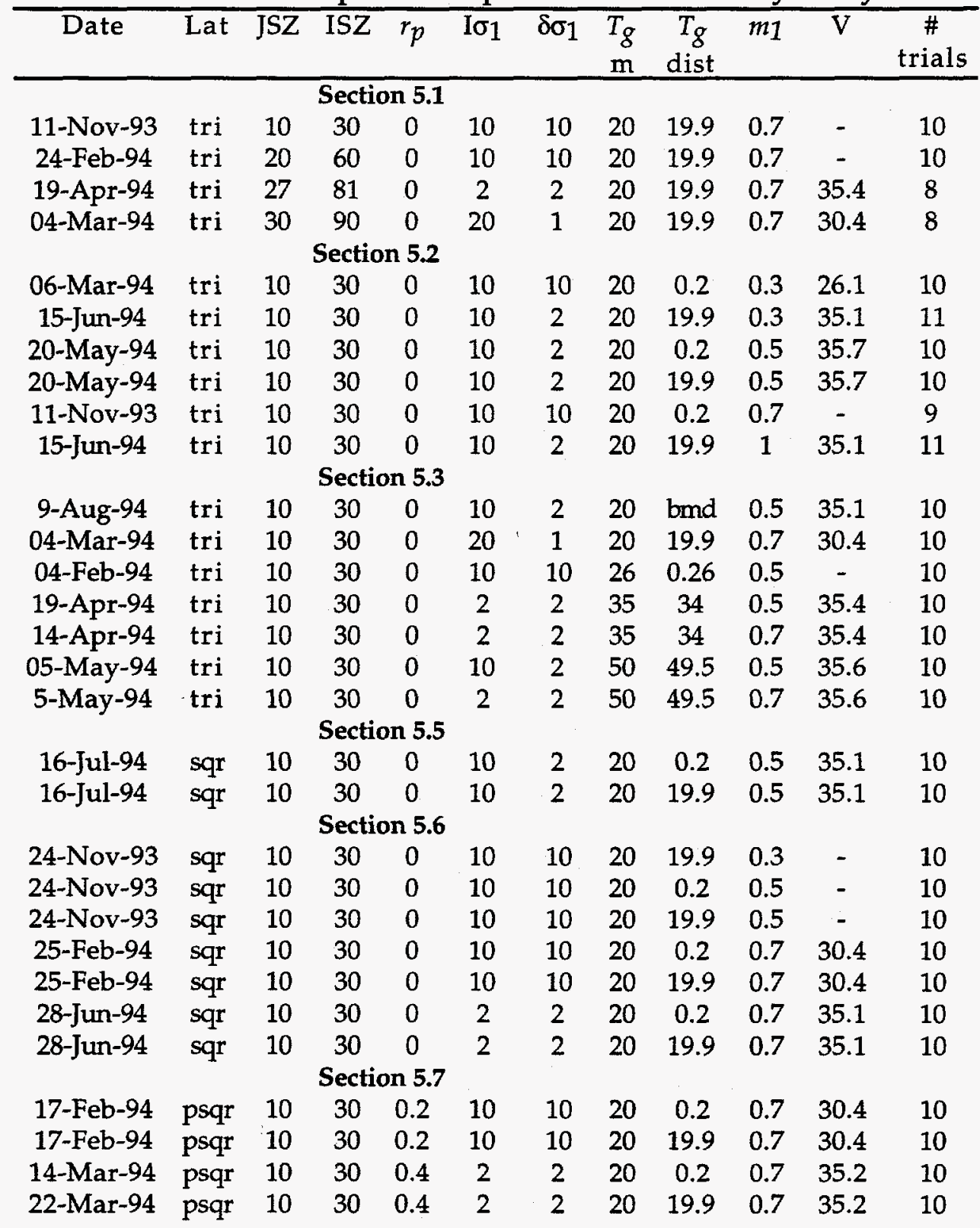


Trials computed for Section 5.1: Lattice Size

Section 5.1.2 10x30

\begin{tabular}{ccccccccccccc}
\hline Date & Lat & JSZ & ISZ & $\mathrm{I} \sigma_{1}$ & $\delta \sigma_{1}$ & $\begin{array}{c}T_{g} \\
\mathrm{~m}\end{array}$ & $\begin{array}{c}T_{g} \\
\text { dist }\end{array}$ & $m_{1}$ & $\mathrm{~V}$ & seq & $\begin{array}{c}\sigma_{p k} \\
(\mathrm{MPa})\end{array}$ & $p_{\mathcal{C}}$ \\
\hline $11-N o v-93$ & tri & 10 & 30 & 10 & 10 & 20 & 19.9 & 0.7 & - & 1 & 31.1 & 0.31 \\
$11-N o v-93$ & $\operatorname{tri}$ & 10 & 30 & 10 & 10 & 20 & 19.9 & 0.7 & - & 2 & 36.8 & 0.34 \\
$11-N o v-93$ & $\operatorname{tri}$ & 10 & 30 & 10 & 10 & 20 & 19.9 & 0.7 & - & 3 & 31.2 & 0.27 \\
$11-N o v-93$ & $\operatorname{tri}$ & 10 & 30 & 10 & 10 & 20 & 19.9 & 0.7 & - & 4 & 31.0 & 0.31 \\
$11-N o v-93$ & $\operatorname{tri}$ & 10 & 30 & 10 & 10 & 20 & 19.9 & 0.7 & - & 5 & 38.4 & 0.33 \\
$11-N o v-93$ & $\operatorname{tri}$ & 10 & 30 & 10 & 10 & 20 & 19.9 & 0.7 & - & 6 & 37.6 & 0.29 \\
$11-N o v-93$ & $\operatorname{tri}$ & 10 & 30 & 10 & 10 & 20 & 19.9 & 0.7 & - & 7 & 33.7 & 0.37 \\
$11-N o v-93$ & $\operatorname{tri}$ & 10 & 30 & 10 & 10 & 20 & 19.9 & 0.7 & - & 8 & 30.8 & 0.27 \\
$11-N o v-93$ & $\operatorname{tri}$ & 10 & 30 & 10 & 10 & 20 & 19.9 & 0.7 & - & 9 & 31.5 & 0.29 \\
11-Nov-93 & tri & 10 & 30 & 10 & 10 & 20 & 19.9 & 0.7 & - & 10 & 39.2 & 0.30 \\
& & & & & & & & & & Mean & 34.1 & 0.31 \\
& & & & & & & & & & Std & 3.3 & 0.03
\end{tabular}

Section 5.1.2 20x60

\begin{tabular}{|c|c|c|c|c|c|c|c|c|c|c|c|c|}
\hline Date & Lat & JSZ & ISZ & $\mathrm{I} \sigma_{1}$ & $\delta \sigma_{1}$ & $\begin{array}{l}T_{g} \\
\text { m }\end{array}$ & $\begin{array}{c}T_{g} \\
\text { dist }\end{array}$ & $m_{1}$ & V & seq & $\begin{array}{c}\sigma_{p k} \\
(\mathrm{MPa})\end{array}$ & $p_{\mathcal{C}}$ \\
\hline 24-Feb-94 & $\operatorname{tri}$ & 20 & 60 & 10 & 10 & 20 & 19.9 & 0.7 & - & 0 & 31.7 & 0.26 \\
\hline 24-Feb-94 & tri & 20 & 60 & 10 & 10 & 20 & 19.9 & 0.7 & - & & 32.0 & 0.26 \\
\hline 24-Feb-94 & tri & 20 & 60 & 10 & 10 & 20 & 19.9 & 0.7 & - & 2 & 32.0 & 0.32 \\
\hline 24-Feb-94 & tri & 20 & 60 & 10 & 10 & 20 & 19.9 & 0.7 & - & 3 & 28.0 & 0.18 \\
\hline 24-Feb-94 & $\operatorname{tri}$ & 20 & 60 & 10 & 10 & 20 & 19.9 & 0.7 & - & 4 & 31.7 & 0.29 \\
\hline 24-Feb-94 & $\operatorname{tri}$ & 20 & 60 & 10 & 10 & 20 & 19.9 & 0.7 & - & 5 & 29.3 & 0.22 \\
\hline 24-Feb-94 & tri & 20 & 60 & 10 & 10 & 20 & 19.9 & 0.7 & - & 6 & 31.1 & 0.20 \\
\hline 24-Feb-94 & $\operatorname{tri}$ & 20 & 60 & 10 & 10 & 20 & 19.9 & 0.7 & - & 7 & 32.0 & 0.22 \\
\hline 24-Feb-94 & tri & 20 & 60 & 10 & 10 & 20 & 19.9 & 0.7 & - & 8 & 32.1 & 0.34 \\
\hline 24-Feb-94 & tri & 20 & 60 & 10 & 10 & 20 & 19.9 & 0.7 & - & 9 & 30.7 & 0.22 \\
\hline & & & & & & & & & & Mean & 31.1 & 0.25 \\
\hline & & & & & & & & & & Std & 1.3 & 0.05 \\
\hline
\end{tabular}

Section 5.1.2 27x81

\begin{tabular}{ccccccccccccc}
\hline Date & Lat & JSZ & ISZ & I $\sigma_{1}$ & $\delta \sigma_{1}$ & $\begin{array}{c}T_{g} \\
\text { m }\end{array}$ & $\begin{array}{c}T_{g} \\
\text { dist }\end{array}$ & $m_{1}$ & V & seq & $\begin{array}{c}\sigma_{p k} \\
(\mathrm{MPa})\end{array}$ & $p_{\mathcal{C}}$ \\
\hline 19-Apr-94 & tri & 27 & 81 & 2 & 2 & 20 & 19.9 & 0.7 & 35.4 & 0 & 21.0 & \\
19-Apr-94 & tri & 27 & 81 & 2 & 2 & 20 & 19.9 & 0.7 & 35.4 & 1 & 24.9 & 0.24 \\
19-Apr-94 & tri & 27 & 81 & 2 & 2 & 20 & 19.9 & 0.7 & 35.4 & 2 & 25.8 & 0.20 \\
19-Apr-94 & tri & 27 & 81 & 2 & 2 & 20 & 19.9 & 0.7 & 35.4 & 3 & 23.3 & 0.23 \\
19-Apr-94 & tri & 27 & 81 & 2 & 2 & 20 & 19.9 & 0.7 & 35.4 & 4 & 25.2 & 0.20 \\
19-Apr-94 & tri & 27 & 81 & 2 & 2 & 20 & 19.9 & 0.7 & 35.4 & 5 & 25.5 & \\
19-Apr-94 & tri & 27 & 81 & 2 & 2 & 20 & 19.9 & 0.7 & 35.4 & 6 & 25.3 & \\
19-Apr-94 & tri & 27 & 81 & 2 & 2 & 20 & 19.9 & 0.7 & 35.4 & 7 & 26.1 & 0.24 \\
& & & & & & & & & & Mean & 24.6 & 0.22 \\
& & & & & & & & & & Std & 1.6 & 0.02
\end{tabular}


Section 5.1.2 30x90

\begin{tabular}{|c|c|c|c|c|c|c|c|c|c|c|c|c|}
\hline Date & Lat & JSZ & ISZ & I $\sigma_{1}$ & $\delta \sigma_{1}$ & $\begin{array}{l}T_{g} \\
\mathrm{~m}\end{array}$ & $\begin{array}{r}T_{g} \\
\text { dist } \\
\end{array}$ & $m_{1}$ & V & seq & $\begin{array}{c}\sigma_{p k} \\
(\mathrm{MPa})\end{array}$ & $p_{C}$ \\
\hline 04-Mar-94 & tri & 30 & 90 & 20 & 1 & 20 & 19.9 & 0.7 & 30.4 & 0 & 23.2 & 0.18 \\
\hline 04-Mar-94 & $\operatorname{tri}$ & 30 & 90 & 20 & 1 & 20 & 19.9 & 0.7 & 30.4 & 1 & 27.3 & \\
\hline 04-Mar-94 & tri & 30 & 90 & 20 & 1 & 20 & 19.9 & 0.7 & 30.4 & 2 & 25.7 & \\
\hline 11-Mar-94 & tri & 30 & 90 & 20 & 1 & 20 & 19.9 & 0.7 & 30.4 & 5 & 23.0 & \\
\hline 11-Mar-94 & tri & 30 & 90 & 20 & 1 & 20 & 19.9 & 0.7 & 30.4 & 6 & 23.4 & \\
\hline 11-Mar-94 & $\operatorname{tri}$ & 30 & 90 & 20 & 1 & 20 & 19.9 & 0.7 & 30.4 & 7 & 22.2 & 0.18 \\
\hline 11-Mar-94 & $\operatorname{tri}$ & 30 & 90 & 20 & 1 & 20 & 19.9 & 0.7 & 30.4 & 8 & 16.9 & 0.18 \\
\hline 11-Mar-94 & tri & 30 & 90 & 20 & 1 & 20 & 19.9 & 0.7 & 30.4 & 9 & 19.2 & \\
\hline & & & & & & & & & & $\begin{array}{c}\text { Mean } \\
\text { Std }\end{array}$ & $\begin{array}{c}22.6 \\
3.1\end{array}$ & $\begin{array}{l}0.18 \\
0.00\end{array}$ \\
\hline
\end{tabular}


Trials computed for Section 5.2: Local Stress Heterogeneity for Triangular Lattice.

Section 5.2.2 $T_{g}$ in $20 \pm 0.2, m_{1}$ in \pm 0.3

\begin{tabular}{ccccccccccccc}
\hline Date & Lat & JSZ & ISZ & I $\sigma_{1}$ & $\delta \sigma_{1}$ & $\begin{array}{c}T_{g} \\
\text { m }\end{array}$ & $\begin{array}{c}T_{g} \\
\text { dist }\end{array}$ & $m_{1}$ & $\mathrm{~V}$ & seq & $\begin{array}{c}\sigma_{p k} \\
(\mathrm{MPa})\end{array}$ & $p_{c}$ \\
\hline 06-Mar-94 & tri & 10 & 30 & 10 & 10 & 20 & 0.2 & 0.3 & 26.1 & 0 & 74.8 & 0.20 \\
06-Mar-94 & tri & 10 & 30 & 10 & 10 & 20 & 0.2 & 0.3 & 26.1 & 1 & 74.8 & 0.28 \\
06-Mar-94 & tri & 10 & 30 & 10 & 10 & 20 & 0.2 & 0.3 & 26.1 & 2 & 74.8 & 0.24 \\
06-Mar-94 & tri & 10 & 30 & 10 & 10 & 20 & 0.2 & 0.3 & 26.1 & 3 & 74.8 & 0.21 \\
06-Mar-94 & tri & 10 & 30 & 10 & 10 & 20 & 0.2 & 0.3 & 26.1 & 4 & 74.8 & 0.18 \\
06-Mar-94 & tri & 10 & 30 & 10 & 10 & 20 & 0.2 & 0.3 & 26.1 & 5 & 74.8 & 0.25 \\
06-Mar-94 & tri & 10 & 30 & 10 & 10 & 20 & 0.2 & 0.3 & 26.1 & 6 & 74.8 & 0.20 \\
06-Mar-94 & tri & 10 & 30 & 10 & 10 & 20 & 0.2 & 0.3 & 26.1 & 7 & 74.8 & 0.18 \\
06-Mar-94 & tri & 10 & 30 & 10 & 10 & 20 & 0.2 & 0.3 & 26.1 & 8 & 74.8 & 0.23 \\
06-Mar-94 & tri & 10 & 30 & 10 & 10 & 20 & 0.2 & 0.3 & 26.1 & 9 & 74.8 & 0.24 \\
& & & & & & & & & & Mean & 74.8 & 0.22 \\
& & & & & & & & & & Std & 0.0 & 0.03
\end{tabular}

Section 5.2.2 $T_{g}$ in $20 \pm 19.9, m_{1}$ in \pm 0.3

\begin{tabular}{ccccccccccccc}
\hline Date & Lat & JSZ & ISZ & $I_{1} \sigma_{1}$ & $\delta \sigma_{1}$ & $\begin{array}{c}T_{g} \\
\mathrm{~m}\end{array}$ & $\begin{array}{c}T_{g} \\
\text { dist }\end{array}$ & $m_{1}$ & $\mathrm{~V}$ & seq & $\sigma_{p k}$ & $p_{\mathcal{C}}$ \\
\hline 15-Jun-94 & tri & 10 & 30 & 10 & 2 & 20 & 19.9 & 0.3 & 35.1 & 0 & 67.1 & 0.32 \\
15-Jun-94 & tri & 10 & 30 & 10 & 2 & 20 & 19.9 & 0.3 & 35.1 & 1 & 81.4 & 0.37 \\
15-Jun-94 & tri & 10 & 30 & 10 & 2 & 20 & 19.9 & 0.3 & 35.1 & 2 & 73.0 & 0.28 \\
15-Jun-94 & tri & 10 & 30 & 10 & 2 & 20 & 19.9 & 0.3 & 35.1 & 3 & 57.7 & 0.26 \\
15-Jun-94 & tri & 10 & 30 & 10 & 2 & 20 & 19.9 & 0.3 & 35.1 & 4 & 58.6 & 0.24 \\
15-Jun-94 & tri & 10 & 30 & 10 & 2 & 20 & 19.9 & 0.3 & 35.1 & 5 & 101.3 & 0.45 \\
15-Jun-94 & tri & 10 & 30 & 10 & 2 & 20 & 19.9 & 0.3 & 35.1 & 6 & 80.7 & 0.31 \\
15-Jun-94 & tri & 10 & 30 & 10 & 2 & 20 & 19.9 & 0.3 & 35.1 & 7 & 46.4 & 0.27 \\
15-Jun-94 & tri & 10 & 30 & 10 & 2 & 20 & 19.9 & 0.3 & 35.1 & 8 & 68.1 & 0.26 \\
15-Jun-94 & tri & 10 & 30 & 10 & 2 & 20 & 19.9 & 0.3 & 35.1 & 9 & 61.7 & 0.28 \\
15-Jun-94 & tri & 10 & 30 & 10 & 2 & 20 & 19.9 & 0.3 & 35.1 & 10 & 61.7 & 0.28 \\
& & & & & & & & & & Mean & 68.9 & 0.30 \\
& & & & & & & & & & Std & 14.2 & 0.06
\end{tabular}

Section 5.2.2 $T_{g}=20 \pm 0.2, m_{1}$ in \pm 0.5 .

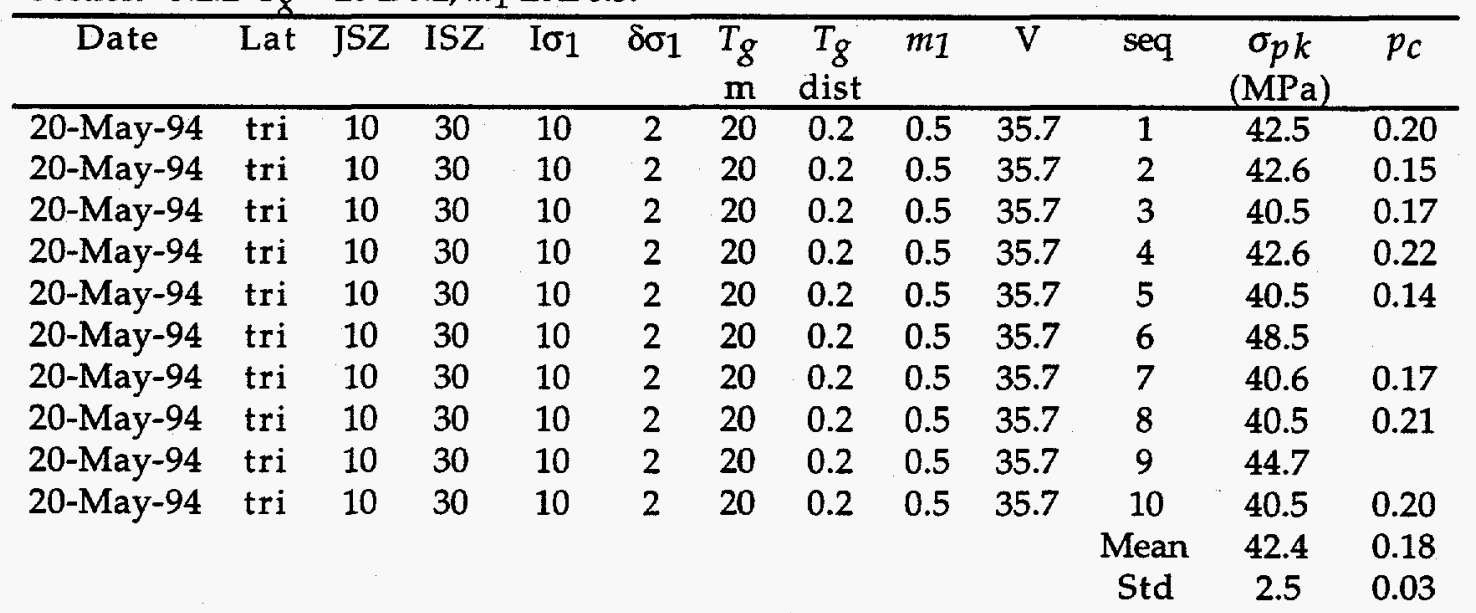


Section 5.2.2 $T_{g}=20 \pm 19.9, m_{1}$ in \pm 0.5 .

\begin{tabular}{ccccccccccccc}
\hline Date & Lat & JSZ & ISZ & $\mathrm{I}_{1}$ & $\delta \sigma_{1}$ & $\begin{array}{c}T_{g} \\
\mathrm{~m}\end{array}$ & $\begin{array}{c}T_{g} \\
\text { dist }\end{array}$ & $m_{1}$ & $\mathrm{~V}$ & seq & $\sigma_{p k}$ & $p_{\mathcal{C}}$ \\
\hline 20-May-94 & tri & 10 & 30 & 10 & 2 & 20 & 19.9 & 0.5 & 35.7 & 1 & 38.5 & 0.29 \\
20-May-94 & $\operatorname{tri}$ & 10 & 30 & 10 & 2 & 20 & 19.9 & 0.5 & 35.7 & 2 & 40.3 & 0.32 \\
$20-$ May-94 & $\operatorname{tri}$ & 10 & 30 & 10 & 2 & 20 & 19.9 & 0.5 & 35.7 & 3 & 42.5 & 0.29 \\
$20-$ May-94 & $\operatorname{tri}$ & 10 & 30 & 10 & 2 & 20 & 19.9 & 0.5 & 35.7 & 4 & 41.8 & 0.32 \\
$20-$ May-94 & $\operatorname{tri}$ & 10 & 30 & 10 & 2 & 20 & 19.9 & 0.5 & 35.7 & 5 & 38.2 & 0.28 \\
20-May-94 & $\operatorname{tri}$ & 10 & 30 & 10 & 2 & 20 & 19.9 & 0.5 & 35.7 & 6 & 48.0 & \\
20-May-94 & $\operatorname{tri}$ & 10 & 30 & 10 & 2 & 20 & 19.9 & 0.5 & 35.7 & 7 & 38.7 & 0.28 \\
20-May-94 & $\operatorname{tri}$ & 10 & 30 & 10 & 2 & 20 & 19.9 & 0.5 & 35.7 & 8 & 42.9 & 0.29 \\
20-May-94 & $\operatorname{tri}$ & 10 & 30 & 10 & 2 & 20 & 19.9 & 0.5 & 35.7 & 9 & 43.1 & 0.31 \\
20-May-94 & $\operatorname{tri}$ & 10 & 30 & 10 & 2 & 20 & 19.9 & 0.5 & 35.7 & 10 & 40.3 & 0.23 \\
& & & & & & & & & & Mean & 41.4 & 0.29 \\
& & & & & & & & & & Std & 2.8 & 0.03
\end{tabular}

Section 5.2.2 $T_{g}=20 \pm 0.2, m_{1}$ in \pm 0.7

\begin{tabular}{ccccccccccccc}
\hline Date & Lat & JSZ & ISZ & I $\sigma_{1}$ & $\delta \sigma_{1}$ & $\begin{array}{c}T_{g} \\
\mathrm{~m}\end{array}$ & $\begin{array}{c}T_{g} \\
\text { dist }\end{array}$ & $m_{1}$ & $\mathrm{~V}$ & seq & $\sigma_{p k}$ & $p_{C}$ \\
\hline $11-N o v-93$ & $\operatorname{tri}$ & 10 & 30 & 10 & 10 & 20 & 0.2 & 0.7 & - & 0 & 31.4 & 0.21 \\
$11-N o v-93$ & $\operatorname{tri}$ & 10 & 30 & 10 & 10 & 20 & 0.2 & 0.7 & - & 1 & 31.4 & 0.28 \\
$11-N o v-93$ & $\operatorname{tri}$ & 10 & 30 & 10 & 10 & 20 & 0.2 & 0.7 & - & 2 & 31.4 & 0.17 \\
$11-N o v-93$ & $\operatorname{tri}$ & 10 & 30 & 10 & 10 & 20 & 0.2 & 0.7 & - & 3 & 31.4 & 0.22 \\
$11-N o v-93$ & $\operatorname{tri}$ & 10 & 30 & 10 & 10 & 20 & 0.2 & 0.7 & - & 4 & 31.4 & 0.19 \\
$11-N o v-93$ & $\operatorname{tri}$ & 10 & 30 & 10 & 10 & 20 & 0.2 & 0.7 & - & 5 & 31.4 & 0.32 \\
$11-N o v-93$ & $\operatorname{tri}$ & 10 & 30 & 10 & 10 & 20 & 0.2 & 0.7 & - & 6 & 31.4 & 0.21 \\
$11-N o v-93$ & $\operatorname{tri}$ & 10 & 30 & 10 & 10 & 20 & 0.2 & 0.7 & - & 7 & 31.4 & 0.19 \\
$11-N o v-93$ & $\operatorname{tri}$ & 10 & 30 & 10 & 10 & 20 & 0.2 & 0.7 & - & 8 & 31.4 & 0.18 \\
& & & & & & & & & & & & \\
& & & & & & & & & & Mean & 31.4 & 0.22 \\
& & & & & & & & & & Std & 0.0 & 0.05
\end{tabular}

Note: for $T_{g}=20 \pm 19.9, m_{1}$ in \pm 0.7 see data for Section 5.1

Section 5.2.2 $T_{g}=20 \pm 19.9, m_{1}$ in \pm 1.0

\begin{tabular}{|c|c|c|c|c|c|c|c|c|c|c|c|c|}
\hline Date & Lat & $\overline{\mathrm{JSZ}}$ & ISZ & $I_{1}$ & $\delta \sigma_{1}$ & $\begin{array}{l}T_{g} \\
\mathrm{~m}\end{array}$ & $\begin{array}{c}T_{g} \\
\text { dist }\end{array}$ & $m_{1}$ & V & seq & $\sigma_{p k}$ & $p_{\mathcal{C}}$ \\
\hline 15-Jun-94 & $\operatorname{tri}$ & 10 & 30 & 10 & 2 & 20 & 19.9 & 1 & 35.1 & 0 & 24.2 & 0.36 \\
\hline 15-Jun-94 & tri & 10 & 30 & 10 & 2 & 20 & 19.9 & 1 & 35.1 & 1 & 30.2 & 0.43 \\
\hline 15-Jun-94 & tri & 10 & 30 & 10 & 2 & 20 & 19.9 & 1 & 35.1 & 2 & 41.4 & 0.52 \\
\hline 15-Jun-94 & tri & 10 & 30 & 10 & 2 & 20 & 19.9 & 1 & 35.1 & 3 & 21.1 & 0.28 \\
\hline 15-Jun-94 & tri & 10 & 30 & 10 & 2 & 20 & 19.9 & 1 & 35.1 & 4 & 17.0 & 0.29 \\
\hline 15-Jun-94 & tri & 10 & 30 & 10 & 2 & 20 & 19.9 & 1 & 35.1 & 5 & 23.4 & 0.27 \\
\hline 15-Jun-94 & $\operatorname{tri}$ & 10 & 30 & 10 & 2 & 20 & 19.9 & 1 & 35.1 & 6 & 18.6 & 0.29 \\
\hline 15-Jun-94 & tri & 10 & 30 & 10 & 2 & 20 & 19.9 & 1 & 35.1 & 7 & 16.8 & 0.32 \\
\hline 15-Jun-94 & $\operatorname{tri}$ & 10 & 30 & 10 & 2 & 20 & 19.9 & 1 & 35.1 & 8 & 17.3 & 0.27 \\
\hline 15-Jun-94 & tri & 10 & 30 & 10 & 2 & 20 & 19.9 & 1 & 35.1 & 9 & 16.8 & 0.29 \\
\hline \multirow[t]{3}{*}{ 15-Jun-94 } & & 10 & 30 & 10 & 2 & 20 & 19.9 & 1 & 35.1 & 10 & 16.8 & 0.29 \\
\hline & & & & & & & & & & Mean & 22.2 & 0.33 \\
\hline & & & & & & & & & & Std & 7.3 & 0.08 \\
\hline
\end{tabular}


Trials computed for Section 5.3: Site Strength Heterogeneity for Triangular

Lattices. Note: additional trials are included in listings for Sections 5.1 and 5.2.

Section 5.3.3 Tg $=20$ (bimodal), $m_{1}$ in \pm 0.5

\begin{tabular}{ccccccccccccc}
\hline Date & Lat & JSZ & ISZ & I $\sigma_{1}$ & $\delta \sigma_{1}$ & $\begin{array}{c}T_{g} \\
\text { m }\end{array}$ & $\begin{array}{c}T_{g} \\
\text { dist }\end{array}$ & $m_{1}$ & $\mathrm{~V}$ & seq & $\sigma_{p k}$ & $p_{\mathcal{C}}$ \\
\hline 9-Aug-94 & tri & 10 & 30 & 10 & 2 & 20 & bmd & 0.5 & 35.1 & 0 & 40.5 & 0.27 \\
9-Aug-94 & tri & 10 & 30 & 10 & 2 & 20 & bmd & 0.5 & 35.1 & 1 & 49.2 & 0.35 \\
9-Aug-94 & tri & 10 & 30 & 10 & 2 & 20 & bmd & 0.5 & 35.1 & 2 & 99.8 & 0.51 \\
9-Aug-94 & tri & 10 & 30 & 10 & 2 & 20 & bmd & 0.5 & 35.1 & 3 & $\mathbf{4 4 . 5}$ & 0.30 \\
9-Aug-94 & tri & 10 & 30 & 10 & 2 & 20 & bmd & 0.5 & 35.1 & 4 & 39.4 & 0.33 \\
9-Aug-94 & tri & 10 & 30 & 10 & 2 & 20 & bmd & 0.5 & 35.1 & 5 & 46.7 & 0.28 \\
9-Aug-94 & tri & 10 & 30 & 10 & 2 & 20 & bmd & 0.5 & 35.1 & 6 & 34.0 & 0.23 \\
9-Aug-94 & tri & 10 & 30 & 10 & 2 & 20 & bmd & 0.5 & 35.1 & 7 & 44.6 & 0.26 \\
9-Aug-94 & tri & 10 & 30 & 10 & 2 & 20 & bmd & 0.5 & 35.1 & 8 & 41.9 & 0.31 \\
9-Aug-94 & tri & 10 & 30 & 10 & 2 & 20 & bmd & 0.5 & 35.1 & 9 & $\mathbf{4 4 . 9}$ & 0.29 \\
& & & & & & & & & & Mean & $\mathbf{4 8 . 6}$ & 0.31 \\
& & & & & & & & & & Std & 17.6 & 0.07
\end{tabular}

Section 5.3.4 $T_{g}=20 \pm 19.9, m_{1}$ in \pm 0.7

\begin{tabular}{ccccccccccccc}
\hline Date & Lat & JSZ & ISZ & $I_{1} \sigma_{1}$ & $\delta \sigma_{1}$ & $\begin{array}{c}T_{g} \\
\mathrm{~m}\end{array}$ & $\begin{array}{c}T_{g} \\
\text { dist }\end{array}$ & $m_{1}$ & $\mathrm{~V}$ & seq & $\sigma_{p k}$ & $p_{c}$ \\
\hline 04-Mar-94 & $\operatorname{tri}$ & 10 & 30 & 20 & 1 & 20 & 19.9 & 0.7 & 30.4 & 0 & 33.7 & 0.31 \\
04-Mar-94 & $\operatorname{tri}$ & 10 & 30 & 20 & 1 & 20 & 19.9 & 0.7 & 30.4 & 1 & 33.8 & 0.34 \\
04-Mar-94 & $\operatorname{tri}$ & 10 & 30 & 20 & 1 & 20 & 19.9 & 0.7 & 30.4 & 2 & 34.3 & 0.33 \\
04-Mar-94 & $\operatorname{tri}$ & 10 & 30 & 20 & 1 & 20 & 19.9 & 0.7 & 30.4 & 3 & 28.2 & 0.36 \\
04-Mar-94 & $\operatorname{tri}$ & 10 & 30 & 20 & 1 & 20 & 19.9 & 0.7 & 30.4 & 4 & 48.5 & 0.49 \\
04-Mar-94 & $\operatorname{tri}$ & 10 & 30 & 20 & 1 & 20 & 19.9 & 0.7 & 30.4 & 5 & 28.9 & 0.31 \\
04-Mar-94 & $\operatorname{tri}$ & 10 & 30 & 20 & 1 & 20 & 19.9 & 0.7 & 30.4 & 6 & 24.3 & 0.28 \\
04-Mar-94 & $\operatorname{tri}$ & 10 & 30 & 20 & 1 & 20 & 19.9 & 0.7 & 30.4 & 7 & 31.4 & 0.37 \\
04-Mar-94 & $\operatorname{tri}$ & 10 & 30 & 20 & 1 & 20 & 19.9 & 0.7 & 30.4 & 8 & 28.4 & 0.28 \\
04-Mar-94 & $\operatorname{tri}$ & 10 & 30 & 20 & 1 & 20 & 19.9 & 0.7 & 30.4 & 9 & 31.1 & 0.32 \\
& & & & & & & & & & Mean & 32.3 & 0.34 \\
& & & & & & & & & & Std & 6.2 & 0.06
\end{tabular}

Section 5.3.4 $T g=26 \pm 0.26, m_{1}$ in \pm 0.5

\begin{tabular}{|c|c|c|c|c|c|c|c|c|c|c|c|c|}
\hline Date & Lat & JSZ & ISZ & $\mathrm{I} \sigma_{1}$ & $\delta \sigma_{1}$ & $\begin{array}{l}T_{g} \\
\mathrm{~m} \\
\end{array}$ & $\begin{array}{c}T_{g} \\
\text { dist }\end{array}$ & $m_{1}$ & V & seq & $\begin{array}{c}\sigma_{p k} \\
(\mathrm{MPa})\end{array}$ & $p_{C}$ \\
\hline 04-Feb-94 & tri & 10 & 30 & 10 & 10 & 26 & 0.26 & 0.5 & - & 0 & 53.4 & 0.30 \\
\hline 04-Feb-94 & $\operatorname{tri}$ & 10 & 30 & 10 & 10 & 26 & 0.26 & 0.5 & - & 1 & 53.4 & 0.17 \\
\hline 04-Feb-94 & tri & 10 & 30 & 10 & 10 & 26 & 0.26 & 0.5 & - & 2 & 64.1 & 0.19 \\
\hline 04-Feb-94 & tri & 10 & 30 & 10 & 10 & 26 & 0.26 & 0.5 & - & 3 & 53.4 & 0.16 \\
\hline 04-Feb-94 & tri & 10 & 30 & 10 & 10 & 26 & 0.26 & 0.5 & - & 4 & 53.4 & 0.22 \\
\hline 04-Feb-94 & $\operatorname{tri}$ & 10 & 30 & 10 & 10 & 26 & 0.26 & 0.5 & - & 5 & 53.4 & 0.28 \\
\hline 04-Feb-94 & $\operatorname{tri}$ & 10 & 30 & 10 & 10 & 26 & 0.26 & 0.5 & - & 6 & 53.4 & 0.19 \\
\hline 04-Feb-94 & $\operatorname{tri}$ & 10 & 30 & 10 & 10 & 26 & 0.26 & 0.5 & - & 7 & 53.4 & 0.22 \\
\hline 04-Feb-94 & tri & 10 & 30 & 10 & 10 & 26 & 0.26 & 0.5 & - & 8 & 58.6 & 0.25 \\
\hline 04-Feb-94 & $\operatorname{tri}$ & 10 & 30 & 10 & 10 & 26 & 0.26 & 0.5 & - & 9 & 53.4 & 0.19 \\
\hline & & & & & & & & & & Mean & 55.0 & 0.22 \\
\hline & & & & & & & & & & Std & 3.4 & 0.05 \\
\hline
\end{tabular}


Secion 5.3.4 $T_{g}=35 \pm 34, m_{1}$ in \pm 0.5

\begin{tabular}{ccccccccccccc}
\hline Date & Lat & JSZ & ISZ & I $\sigma_{1}$ & $\delta \sigma_{1}$ & $\begin{array}{c}T_{g} \\
\mathrm{~m}\end{array}$ & $\begin{array}{c}T_{g} \\
\text { dist }\end{array}$ & $m_{1}$ & $\mathrm{~V}$ & seq & $\sigma_{p k}$ & $p_{\mathcal{C}}$ \\
\hline 19-Apr-94 & tri & 10 & 30 & 2 & 2 & 35 & 34 & 0.5 & 35.4 & 0 & 54.1 & 0.30 \\
19-Apr-94 & tri & 10 & 30 & 2 & 2 & 35 & 34 & 0.5 & 35.4 & 1 & 76.3 & 0.39 \\
19-Apr-94 & tri & 10 & 30 & 2 & 2 & 35 & 34 & 0.5 & 35.4 & 2 & 79.8 & 0.32 \\
19-Apr-94 & tri & 10 & 30 & 2 & 2 & 35 & 34 & 0.5 & 35.4 & 3 & 58.3 & 0.27 \\
19-Apr-94 & tri & 10 & 30 & 2 & 2 & 35 & 34 & 0.5 & 35.4 & 4 & 55.0 & 0.31 \\
19-Apr-94 & tri & 10 & 30 & 2 & 2 & 35 & 34 & 0.5 & 35.4 & 5 & 69.3 & 0.36 \\
19-Apr-94 & tri & 10 & 30 & 2 & 2 & 35 & 34 & 0.5 & 35.4 & 6 & 72.8 & 0.27 \\
19-Apr-94 & tri & 10 & 30 & 2 & 2 & 35 & 34 & 0.5 & 35.4 & 7 & 73.9 & 0.30 \\
19-Apr-94 & tri & 10 & 30 & 2 & 2 & 35 & 34 & 0.5 & 35.4 & 8 & 69.5 & 0.34 \\
19-Apr-94 & tri & 10 & 30 & 2 & 2 & 35 & 34 & 0.5 & 35.4 & 9 & 69.4 & 0.24 \\
& & & & & & & & & & Mean & 67.8 & 0.31 \\
& & & & & & & & & & Std & 8.5 & 0.04
\end{tabular}

Section 5.2.2 $T_{g}=35 \pm 34, m_{1}$ in \pm 0.7

\begin{tabular}{ccccccccccccc}
\hline Date & Lat & JSZ & ISZ & Is1 & ds1 & $\begin{array}{c}T_{g} \\
\mathrm{~m}\end{array}$ & $\begin{array}{c}T_{g} \\
\text { dist }\end{array}$ & $m_{1}$ & $\mathrm{~V}$ & seq & $\begin{array}{c}s p k \\
(\mathrm{MPa})\end{array}$ & $p_{\mathcal{C}}$ \\
\hline 14-Apr-94 & tri & 10 & 30 & 2 & 2 & 35 & 34 & 0.7 & 35.4 & 0 & 46.8 & 0.35 \\
14-Apr-94 & tri & 10 & 30 & 2 & 2 & 35 & 34 & 0.7 & 35.4 & 1 & 56.5 & 0.31 \\
14-Apr-94 & tri & 10 & 30 & 2 & 2 & 35 & 34 & 0.7 & 35.4 & 2 & 64.8 & 0.38 \\
14-Apr-94 & tri & 10 & 30 & 2 & 2 & 35 & 34 & 0.7 & 35.4 & 3 & 46.9 & 0.28 \\
14-Apr-94 & tri & 10 & 30 & 2 & 2 & 35 & 34 & 0.7 & 35.4 & 4 & 53.8 & 0.28 \\
14-Apr-94 & tri & 10 & 30 & 2 & 2 & 35 & 34 & 0.7 & 35.4 & 5 & 50.4 & 0.29 \\
14-Apr-94 & tri & 10 & 30 & 2 & 2 & 35 & 34 & 0.7 & 35.4 & 6 & 49.8 & 0.39 \\
14-Apr-94 & tri & 10 & 30 & 2 & 2 & 35 & 34 & 0.7 & 35.4 & 7 & 47.5 & 0.27 \\
14-Apr-94 & tri & 10 & 30 & 2 & 2 & 35 & 34 & 0.7 & 35.4 & 8 & 36.3 & 0.24 \\
14-Apr-94 & tri & 10 & 30 & 2 & 2 & 35 & 34 & 0.7 & 35.4 & 9 & 51.1 & 0.36 \\
& & & & & & & & & & Mean & 50.4 & 0.32 \\
& & & & & & & & & & Std & 7.0 & 0.05
\end{tabular}

Section 5.3.4 $T_{g}=50 \pm 49.5, m_{1}$ in \pm 0.5

\begin{tabular}{ccccccccccccc}
\hline Date & Lat & JSZ & ISZ & I $\sigma_{1}$ & $\delta \sigma_{1}$ & $\begin{array}{c}T_{g} \\
\mathrm{~m}\end{array}$ & $\begin{array}{c}T_{g} \\
\text { dist }\end{array}$ & $m_{1}$ & $\mathrm{~V}$ & seq & $\sigma_{p k}$ & $p_{\mathcal{C}}$ \\
\hline 05-May-94 & tri & 10 & 30 & 10 & 2 & 50 & 49.5 & 0.5 & 35.6 & 0 & 97.4 & 0.28 \\
05-May-94 & tri & 10 & 30 & 10 & 2 & 50 & 49.5 & 0.5 & 35.6 & 1 & 145.4 & 0.40 \\
05-May-94 & tri & 10 & 30 & 10 & 2 & 50 & 49.5 & 0.5 & 35.6 & 2 & 96.4 & 0.24 \\
05-May-94 & $\operatorname{tri}$ & 10 & 30 & 10 & 2 & 50 & 49.5 & 0.5 & 35.6 & 3 & 91.2 & 0.23 \\
05-May-94 & $\operatorname{tri}$ & 10 & 30 & 10 & 2 & 50 & 49.5 & 0.5 & 35.6 & 4 & 147.7 & 0.45 \\
05-May-94 & $\operatorname{tri}$ & 10 & 30 & 10 & 2 & 50 & 49.5 & 0.5 & 35.6 & 5 & 94.8 & 0.33 \\
05-May-94 & $\operatorname{tri}$ & 10 & 30 & 10 & 2 & 50 & 49.5 & 0.5 & 35.6 & 6 & 96.5 & 0.30 \\
05-May-94 & tri & 10 & 30 & 10 & 2 & 50 & 49.5 & 0.5 & 35.6 & 7 & 100.7 & 0.33 \\
05-May-94 & tri & 10 & 30 & 10 & 2 & 50 & 49.5 & 0.5 & 35.6 & 9 & 112.3 & 0.26 \\
& & & & & & & & & & & & \\
& & & & & & & & & & Mean & 109.2 & 0.31 \\
& & & & & & & & & & Std & 20.7 & 0.07
\end{tabular}


Section 5.3.4 $T_{g}=50 \pm 49.5, m_{1}$ in \pm 0.7

\begin{tabular}{ccccccccccccc}
\hline Date & Lat & JSZ & ISZ & I $\sigma_{1}$ & $\delta \sigma_{1}$ & $\begin{array}{c}T_{g} \\
\mathrm{~m}\end{array}$ & $\begin{array}{c}T_{g} \\
\text { dist }\end{array}$ & $m_{1}$ & $\mathrm{~V}$ & seq & $\sigma_{p k}$ & $p_{\mathcal{c}}$ \\
\hline 5-May-94 & tri & 10 & 30 & 2 & 2 & 50 & 49.5 & 0.7 & 35.6 & 0 & 73.7 & 0.28 \\
5-May-94 & tri & 10 & 30 & 2 & 2 & 50 & 49.5 & 0.7 & 35.6 & 1 & 58.6 & 0.27 \\
5-May-94 & tri & 10 & 30 & 2 & 2 & 50 & 49.5 & 0.7 & 35.6 & 2 & 77.4 & 0.34 \\
5-May-94 & tri & 10 & 30 & 2 & 2 & 50 & 49.5 & 0.7 & 35.6 & 3 & 74.3 & 0.39 \\
5-May-94 & tri & 10 & 30 & 2 & 2 & 50 & 49.5 & 0.7 & 35.6 & 4 & 79.7 & 0.27 \\
5-May-94 & tri & 10 & 30 & 2 & 2 & 50 & 49.5 & 0.7 & 35.6 & 5 & 83.7 & 0.38 \\
5-May-94 & tri & 10 & 30 & 2 & 2 & 50 & 49.5 & 0.7 & 35.6 & 6 & 63.4 & 0.29 \\
5-May-94 & tri & 10 & 30 & 2 & 2 & 50 & 49.5 & 0.7 & 35.6 & 7 & 94.6 & 0.39 \\
5-May-94 & tri & 10 & 30 & 2 & 2 & 50 & 49.5 & 0.7 & 35.6 & 8 & 96.0 & 0.40 \\
5-May-94 & tri & 10 & 30 & 2 & 2 & 50 & 49.5 & 0.7 & 35.6 & 9 & 58.8 & 0.34 \\
& & & & & & & & & & Mean & 76.0 & 0.33 \\
& & & & & & & & & & Std & 12.6 & 0.05
\end{tabular}


Trials computed for Section 5.5: Site Strength Heterogeneity for Square Lattice.

Section 5.5.1 $T_{g}$ in $20 \pm 0.2, m_{1}$ in \pm 0.5

\begin{tabular}{ccccccccccccc}
\hline Date & Lat & JSZ & ISZ & I $\sigma_{1}$ & $\delta \sigma_{1}$ & $\begin{array}{c}T_{g} \\
\text { m }\end{array}$ & $\begin{array}{c}T_{g} \\
\text { dist }\end{array}$ & $m_{1}$ & $\mathrm{~V}$ & seq & $\begin{array}{c}\sigma_{p k} \\
(\mathrm{MPa})\end{array}$ & $p_{c}$ \\
\hline 16-Jul-94 & sqr & 10 & 30 & 10 & 2 & 20 & 0.2 & 0.5 & 35.1 & 0 & 40.5 & 0.16 \\
16-Jul-94 & sqr & 10 & 30 & 10 & 2 & 20 & 0.2 & 0.5 & 35.1 & 1 & 46.9 & 0.14 \\
16-Jul-94 & sqr & 10 & 30 & 10 & 2 & 20 & 0.2 & 0.5 & 35.1 & 2 & 40.5 & 0.17 \\
16-Jul-94 & sqr & 10 & 30 & 10 & 2 & 20 & 0.2 & 0.5 & 35.1 & 3 & 42.6 & 0.13 \\
16-Jul-94 & sqr & 10 & 30 & 10 & 2 & 20 & 0.2 & 0.5 & 35.1 & 4 & 40.5 & 0.15 \\
16-Jul-94 & sqr & 10 & 30 & 10 & 2 & 20 & 0.2 & 0.5 & 35.1 & 5 & 42.6 & 0.13 \\
16-Jul-94 & sqr & 10 & 30 & 10 & 2 & 20 & 0.2 & 0.5 & 35.1 & 6 & 40.5 & 0.13 \\
16-Jul-94 & sqr & 10 & 30 & 10 & 2 & 20 & 0.2 & 0.5 & 35.1 & 7 & 40.5 & 0.17 \\
16-Jul-94 & sqr & 10 & 30 & 10 & 2 & 20 & 0.2 & 0.5 & 35.1 & 8 & 40.5 & 0.14 \\
16-Jul-94 & sqr & 10 & 30 & 10 & 2 & 20 & 0.2 & 0.5 & 35.1 & 9 & 40.5 & 0.14 \\
& & & & & & & & & & Mean & 41.6 & 0.15 \\
& & & & & & & & & & Std & 2.0 & 0.01
\end{tabular}

Secion 5.5.2 $T_{g}$ in $20 \pm 19.9, m_{1}$ in \pm 0.5

\begin{tabular}{ccccccccccccc}
\hline Date & Lat & JSZ & ISZ & I $\sigma_{1}$ & $\delta \sigma_{1}$ & $\begin{array}{c}T_{g} \\
\text { m }\end{array}$ & $\begin{array}{c}T_{g} \\
\text { dist }\end{array}$ & $m_{1}$ & $\mathrm{~V}$ & seq & $\sigma_{p k}$ & $p_{\mathcal{C}}$ \\
\hline 16-Jul-94 & sqr & 10 & 30 & 10 & 2 & 20 & 19.9 & 0.5 & 35.1 & 0 & 32.0 & 0.24 \\
16-Jul-94 & sqr & 10 & 30 & 10 & 2 & 20 & 19.9 & 0.5 & 35.1 & 1 & 33.9 & 0.23 \\
16-Jul-94 & sqr & 10 & 30 & 10 & 2 & 20 & 19.9 & 0.5 & 35.1 & 2 & 33.0 & 0.27 \\
16-Jul-94 & sqr & 10 & 30 & 10 & 2 & 20 & 19.9 & 0.5 & 35.1 & 3 & 40.3 & 0.26 \\
16-Jul-94 & sqr & 10 & 30 & 10 & 2 & 20 & 19.9 & 0.5 & 35.1 & 4 & 31.9 & 0.22 \\
16-Jul-94 & sqr & 10 & 30 & 10 & 2 & 20 & 19.9 & 0.5 & 35.1 & 5 & 43.7 & 0.27 \\
16-Jul-94 & sqr & 10 & 30 & 10 & 2 & 20 & 19.9 & 0.5 & 35.1 & 6 & 31.8 & 0.23 \\
16-Jul-94 & sqr & 10 & 30 & 10 & 2 & 20 & 19.9 & 0.5 & 35.1 & 7 & 33.7 & 0.29 \\
16-Jul-94 & sqr & 10 & 30 & 10 & 2 & 20 & 19.9 & 0.5 & 35.1 & 8 & 45.4 & 0.28 \\
16-Jul-94 & sqr & 10 & 30 & 10 & 2 & 20 & 19.9 & 0.5 & 35.1 & 9 & 42.3 & 0.21 \\
& & & & & & & & & & Mean & 36.8 & 0.25 \\
& & & & & & & & & & Std & 5.2 & 0.03
\end{tabular}


Trials computed for Section 5.6: Local Stress Heterogeneity for Square Lattice

Section $5.6 T_{g}$ in $20 \pm 19.9, m_{1}$ in \pm 0.3

\begin{tabular}{cccccccccccccc}
\hline Date & Lat & JSZ & ISZ & $r_{p}$ & $I_{1} \sigma_{1}$ & $\delta \sigma_{1}$ & $\begin{array}{c}T_{g} \\
\text { m }\end{array}$ & $\begin{array}{c}T_{g} \\
\text { dist }\end{array}$ & $m_{1}$ & $V$ & seq & $\begin{array}{c}\sigma_{p k} \\
(\mathrm{MPa})\end{array}$ & $p_{\mathcal{C}}$ \\
\hline 24-Nov-93 & sqr & 10 & 30 & 10 & 10 & 20 & 19.9 & 0.3 & - & 0 & 62.5 & 0.25 \\
$24-N o v-93$ & sqr & 10 & 30 & 10 & 10 & 20 & 19.9 & 0.3 & - & 1 & 73.3 & 0.29 \\
$24-N o v-93$ & sqr & 10 & 30 & 10 & 10 & 20 & 19.9 & 0.3 & - & 2 & 92.4 & 0.33 \\
24-Nov-93 & sqr & 10 & 30 & 10 & 10 & 20 & 19.9 & 0.3 & - & 3 & 63.8 & 0.25 \\
24-Nov-93 & sqr & 10 & 30 & 10 & 10 & 20 & 19.9 & 0.3 & - & 4 & 52.2 & 0.27 \\
24-Nov-93 & sqr & 10 & 30 & 10 & 10 & 20 & 19.9 & 0.3 & - & 5 & 72.6 & 0.32 \\
24-Nov-93 & sqr & 10 & 30 & 10 & 10 & 20 & 19.9 & 0.3 & - & 6 & 48.0 & 0.25 \\
24-Nov-93 & sqr & 10 & 30 & 10 & 10 & 20 & 19.9 & 0.3 & - & 7 & 71.7 & 0.28 \\
24-Nov-93 & sqr & 10 & 30 & 10 & 10 & 20 & 19.9 & 0.3 & - & 8 & 82.6 & 0.33 \\
24-Nov-93 & sqr & 10 & 30 & 10 & 10 & 20 & 19.9 & 0.3 & - & 9 & 71.4 & 0.30 \\
& & & & & & & & & & Mean & 69.1 & 0.29 \\
& & & & & & & & & & Std & 12.5 & 0.03
\end{tabular}

Section $5.6 T_{g}$ in $20 \pm 0.2, m_{1}$ in \pm 0.5

\begin{tabular}{ccccccccccccc}
\hline Date & Lat & JSZ & ISZ & I $\sigma_{1}$ & $\delta \sigma_{1}$ & $\begin{array}{c}T_{g} \\
\text { m }\end{array}$ & $\begin{array}{c}T_{g} \\
\text { dist }\end{array}$ & $m_{1}$ & $\mathrm{~V}$ & seq & $\sigma_{p k}$ & $p_{c}$ \\
\hline 24-Nov-93 & sqr & 10 & 30 & 10 & 10 & 20 & 0.2 & 0.5 & - & 0 & 42.7 & 0.11 \\
$24-N o v-93$ & sqr & 10 & 30 & 10 & 10 & 20 & 0.2 & 0.5 & - & 1 & 42.7 & 0.13 \\
$24-N o v-93$ & sqr & 10 & 30 & 10 & 10 & 20 & 0.2 & 0.5 & - & 2 & 42.7 & 0.13 \\
$24-N o v-93$ & sqr & 10 & 30 & 10 & 10 & 20 & 0.2 & 0.5 & - & 3 & 42.7 & 0.13 \\
$24-N o v-93$ & sqr & 10 & 30 & 10 & 10 & 20 & 0.2 & 0.5 & - & 4 & 42.7 & 0.19 \\
$24-N o v-93$ & sqr & 10 & 30 & 10 & 10 & 20 & 0.2 & 0.5 & - & 5 & 42.7 & 0.14 \\
$24-N o v-93$ & sqr & 10 & 30 & 10 & 10 & 20 & 0.2 & 0.5 & - & 6 & 42.7 & 0.15 \\
$24-N o v-93$ & sqr & 10 & 30 & 10 & 10 & 20 & 0.2 & 0.5 & - & 7 & 42.7 & 0.15 \\
24-Nov-93 & sqr & 10 & 30 & 10 & 10 & 20 & 0.2 & 0.5 & - & 8 & 42.7 & 0.14 \\
24-Nov-93 & sqr & 10 & 30 & 10 & 10 & 20 & 0.2 & 0.5 & - & 9 & 42.7 & 0.12 \\
& & & & & & & & & & Mean & 42.7 & 0.14 \\
& & & & & & & & & & Std & 0.0 & 0.02
\end{tabular}

Section $5.6 T_{g}$ in $20 \pm 19.9, m_{1}$ in \pm 0.5

\begin{tabular}{ccccccccccccc}
\hline Date & Lat & JSZ & ISZ & $I \sigma_{1}$ & $\delta \sigma_{1}$ & $\begin{array}{c}T_{g} \\
\mathrm{~m}\end{array}$ & $\begin{array}{c}T_{g} \\
\text { dist }\end{array}$ & $m_{1}$ & $\mathrm{~V}$ & seq & $\sigma_{p k}$ & $p_{\mathcal{C}}$ \\
\hline 24-Nov-93 & sqr & 10 & 30 & 10 & 10 & 20 & 19.9 & 0.5 & - & 0 & 31.9 & 0.21 \\
$24-N o v-93$ & sqr & 10 & 30 & 10 & 10 & 20 & 19.9 & 0.5 & - & 1 & 42.4 & 0.39 \\
$24-N o v-93$ & sqr & 10 & 30 & 10 & 10 & 20 & 19.9 & 0.5 & - & 2 & 42.3 & 0.27 \\
$24-N o v-93$ & sqr & 10 & 30 & 10 & 10 & 20 & 19.9 & 0.5 & - & 3 & 24.7 & 0.25 \\
$24-N o v-93$ & sqr & 10 & 30 & 10 & 10 & 20 & 19.9 & 0.5 & - & 4 & 39.5 & 0.22 \\
$24-N o v-93$ & sqr & 10 & 30 & 10 & 10 & 20 & 19.9 & 0.5 & - & 5 & 42.4 & 0.23 \\
$24-N o v-93$ & sqr & 10 & 30 & 10 & 10 & 20 & 19.9 & 0.5 & - & 6 & 31.8 & 0.28 \\
$24-N o v-93$ & sqr & 10 & 30 & 10 & 10 & 20 & 19.9 & 0.5 & - & 7 & 41.0 & 0.23 \\
$24-N o v-93$ & sqr & 10 & 30 & 10 & 10 & 20 & 19.9 & 0.5 & - & 8 & 38.6 & 0.29 \\
$24-N o v-93$ & sqr & 10 & 30 & 10 & 10 & 20 & 19.9 & 0.5 & - & 9 & 31.8 & 0.27 \\
& & & & & & & & & & Mean & 36.6 & 0.26 \\
& & & & & & & & & & Std & 5.9 & 0.05
\end{tabular}


Section $5.6 T_{g}$ in $20 \pm 0.2, m_{1}$ in \pm 0.7

\begin{tabular}{ccccccccccccc}
\hline Date & Lat & JSZ & ISZ & I $\sigma_{1}$ & $\delta \sigma_{1}$ & $\begin{array}{c}T_{g} \\
\mathrm{~m}\end{array}$ & $\begin{array}{c}T_{g} \\
\text { dist }\end{array}$ & $m_{1}$ & $\mathrm{~V}$ & seq & $\begin{array}{c}\sigma_{p k} \\
(\mathrm{MPa})\end{array}$ & $p_{\mathcal{c}}$ \\
\hline 25-Feb-94 & sqr & 10 & 30 & 10 & 10 & 20 & 0.2 & 0.7 & 30.4 & 0 & 40.3 & 0.25 \\
25-Feb-94 & sqr & 10 & 30 & 10 & 10 & 20 & 0.2 & 0.7 & 30.4 & 1 & 32.0 & 0.11 \\
25-Feb-94 & sqr & 10 & 30 & 10 & 10 & 20 & 0.2 & 0.7 & 30.4 & 2 & 42.8 & 0.15 \\
25-Feb-94 & sqr & 10 & 30 & 10 & 10 & 20 & 0.2 & 0.7 & 30.4 & 3 & 32.0 & 0.12 \\
25-Feb-94 & sqr & 10 & 30 & 10 & 10 & 20 & 0.2 & 0.7 & 30.4 & 4 & 32.0 & 0.13 \\
25-Feb-94 & sqr & 10 & 30 & 10 & 10 & 20 & 0.2 & 0.7 & 30.4 & 5 & 32.0 & 0.13 \\
25-Feb-94 & sqr & 10 & 30 & 10 & 10 & 20 & 0.2 & 0.7 & 30.4 & 6 & 32.0 & 0.23 \\
25-Feb-94 & sqr & 10 & 30 & 10 & 10 & 20 & 0.2 & 0.7 & 30.4 & 7 & 32.0 & 0.27 \\
25-Feb-94 & sqr & 10 & 30 & 10 & 10 & 20 & 0.2 & 0.7 & 30.4 & 8 & 32.0 & 0.15 \\
25-Feb-94 & sqr & 10 & 30 & 10 & 10 & 20 & 0.2 & 0.7 & 30.4 & 9 & 32.0 & 0.14 \\
& & & & & & & & & & Mean & 33.9 & 0.17 \\
& & & & & & & & & & Std & 3.9 & 0.06
\end{tabular}

Section 5.6 $T_{g}$ in $20 \pm 19.9, m_{1}$ in \pm 0.7

\begin{tabular}{ccccccccccccc}
\hline Date & Lat & JSZ & ISZ & $\mathrm{I} \sigma_{1}$ & $\delta \sigma_{1}$ & $\begin{array}{c}T_{g} \\
\mathrm{~m}\end{array}$ & $\begin{array}{c}T_{g} \\
\text { dist }\end{array}$ & $m_{1}$ & $\mathrm{~V}$ & seq & $\sigma_{p k}$ & $p_{C}$ \\
\hline 25-Feb-94 & sqr & 10 & 30 & 10 & 10 & 20 & 19.9 & 0.7 & 30.4 & 0 & 31.9 & 0.26 \\
25-Feb-94 & sqr & 10 & 30 & 10 & 10 & 20 & 19.9 & 0.7 & 30.4 & 1 & 31.9 & 0.30 \\
25-Feb-94 & sqr & 10 & 30 & 10 & 10 & 20 & 19.9 & 0.7 & 30.4 & 2 & 31.6 & 0.26 \\
25-Feb-94 & sqr & 10 & 30 & 10 & 10 & 20 & 19.9 & 0.7 & 30.4 & 3 & 31.9 & 0.26 \\
25-Feb-94 & sqr & 10 & 30 & 10 & 10 & 20 & 19.9 & 0.7 & 30.4 & 4 & 30.0 & 0.27 \\
25-Feb-94 & sqr & 10 & 30 & 10 & 10 & 20 & 19.9 & 0.7 & 30.4 & 5 & 28.9 & 0.22 \\
25-Feb-94 & sqr & 10 & 30 & 10 & 10 & 20 & 19.9 & 0.7 & 30.4 & 6 & 34.3 & 0.31 \\
25-Feb-94 & sqr & 10 & 30 & 10 & 10 & 20 & 19.9 & 0.7 & 30.4 & 7 & 29.9 & 0.21 \\
25-Feb-94 & sqr & 10 & 30 & 10 & 10 & 20 & 19.9 & 0.7 & 30.4 & 8 & 31.6 & 0.22 \\
25-Feb-94 & sqr & 10 & 30 & 10 & 10 & 20 & 19.9 & 0.7 & 30.4 & 9 & 31.6 & 0.26 \\
& & & & & & & & & & Mean & 31.4 & 0.26 \\
& & & & & & & & & & Std & 1.4 & 0.03
\end{tabular}

Section $5.6 T_{g}$ in $20 \pm 0.2, m_{1}$ in \pm 0.7

\begin{tabular}{ccccccccccccc}
\hline Date & Lat & JSZ & ISZ & $\mathrm{I}_{1}$ & $\delta \sigma_{1}$ & $\begin{array}{c}T_{g} \\
\mathrm{~m}\end{array}$ & $\begin{array}{c}T_{g} \\
\text { dist }\end{array}$ & $m_{1}$ & $\mathrm{~V}$ & seq & $\sigma_{p k}$ & $p_{\mathcal{C}}$ \\
\hline 28-Jun-94 & sqr & 10 & 30 & 2 & 2 & 20 & 0.2 & 0.7 & 35.1 & 0 & 32.7 & 0.23 \\
28-Jun-94 & sqr & 10 & 30 & 2 & 2 & 20 & 0.2 & 0.7 & 35.1 & 1 & 29.8 & 0.11 \\
28-Jun-94 & sqr & 10 & 30 & 2 & 2 & 20 & 0.2 & 0.7 & 35.1 & 2 & 34.1 & 0.14 \\
28-Jun-94 & sqr & 10 & 30 & 2 & 2 & 20 & 0.2 & 0.7 & 35.1 & 3 & 29.8 & 0.16 \\
28-Jun-94 & sqr & 10 & 30 & 2 & 2 & 20 & 0.2 & 0.7 & 35.1 & 4 & 29.8 & 0.13 \\
28-Jun-94 & sqr & 10 & 30 & 2 & 2 & 20 & 0.2 & 0.7 & 35.1 & 5 & 29.8 & 0.12 \\
28-Jun-94 & sqr & 10 & 30 & 2 & 2 & 20 & 0.2 & 0.7 & 35.1 & 6 & 29.8 & 0.23 \\
28-Jun-94 & sqr & 10 & 30 & 2 & 2 & 20 & 0.2 & 0.7 & 35.1 & 7 & 29.8 & 0.15 \\
28-Jun-94 & sqr & 10 & 30 & 2 & 2 & 20 & 0.2 & 0.7 & 35.1 & 8 & 29.8 & 0.15 \\
28-Jun-94 & sqr & 10 & 30 & 2 & 2 & 20 & 0.2 & 0.7 & 35.1 & 9 & 32.0 & 0.18 \\
& & & & & & & & & & Mean & 30.8 & 0.16 \\
& & & & & & & & & & Std & 1.5 & 0.04
\end{tabular}


Section $5.6 T_{g}$ in $20 \pm 19.9, m_{1}$ in \pm 0.7

\begin{tabular}{cccccccccccccc}
\hline Date & Lat & JSZ & ISZ & $r_{p}$ & $\mathrm{I}_{1}$ & $\delta \sigma_{1}$ & $\begin{array}{c}T_{g} \\
\mathrm{~m}\end{array}$ & $\begin{array}{c}T_{g} \\
\text { dist }\end{array}$ & $m_{1}$ & $\mathrm{~V}$ & seq & $\begin{array}{c}\sigma_{p k} \\
(\mathrm{MPa})\end{array}$ & $p_{\mathcal{C}}$ \\
\hline 28-Jun-94 & sqr & 10 & 30 & 0 & 2 & 2 & 20 & 19.9 & 0.7 & 35.1 & 0 & 26.7 & 0.27 \\
28-Jun-94 & sqr & 10 & 30 & 0 & 2 & 2 & 20 & 19.9 & 0.7 & 35.1 & 1 & 27.8 & 0.32 \\
28-Jun-94 & sqr & 10 & 30 & 0 & 2 & 2 & 20 & 19.9 & 0.7 & 35.1 & 2 & 27.3 & 0.24 \\
28-Jun-94 & sqr & 10 & 30 & 0 & 2 & 2 & 20 & 19.9 & 0.7 & 35.1 & 3 & 31.5 & 0.29 \\
28-Jun-94 & sqr & 10 & 30 & 0 & 2 & 2 & 20 & 19.9 & 0.7 & 35.1 & 4 & 20.5 & 0.23 \\
28-Jun-94 & sqr & 10 & 30 & 0 & 2 & 2 & 20 & 19.9 & 0.7 & 35.1 & 5 & 21.5 & 0.22 \\
28-Jun-94 & sqr & 10 & 30 & 0 & 2 & 2 & 20 & 19.9 & 0.7 & 35.1 & 6 & 37.0 & 0.34 \\
28-Jun-94 & sqr & 10 & 30 & 0 & 2 & 2 & 20 & 19.9 & 0.7 & 35.1 & 7 & 22.3 & 0.22 \\
28-Jun-94 & sqr & 10 & 30 & 0 & 2 & 2 & 20 & 19.9 & 0.7 & 35.1 & 8 & 28.0 & 0.21 \\
28-Jun-94 & sqr & 10 & 30 & 0 & 2 & 2 & 20 & 19.9 & 0.7 & 35.1 & 9 & 26.9 & 0.31 \\
& & & & & & & & & & & Mean & 27.0 & 0.27 \\
& & & & & & & & & & & Std & 4.7 & 0.04
\end{tabular}


Trials Computed for Section 5.7: Perturbed Square Lattices

Section 5.7.3 $T_{g}$ in $20 \pm 0.2, m_{1}$ in $\pm 0.7, r_{p \max }=0.2$

\begin{tabular}{ccccccccccccc}
\hline Date & Lat & JSZ & ISZ & $\mathrm{I} \sigma_{1}$ & $\delta \sigma_{1}$ & $\begin{array}{c}T_{g} \\
\mathrm{~m}\end{array}$ & $\begin{array}{c}T_{g} \\
\text { dist }\end{array}$ & $m_{1}$ & $\mathrm{~V}$ & seq & $\begin{array}{c}\sigma_{p k} \\
(\mathrm{MPa})\end{array}$ & $p_{c}$ \\
\hline 17-Feb-94 & psqr & 10 & 30 & 10 & 10 & 20 & 0.2 & 0.7 & 30.4 & 0 & 32.1 & 0.16 \\
$17-F e b-94$ & psqr & 10 & 30 & 10 & 10 & 20 & 0.2 & 0.7 & 30.4 & 1 & 32.1 & 0.13 \\
$17-F e b-94$ & psqr & 10 & 30 & 10 & 10 & 20 & 0.2 & 0.7 & 30.4 & 2 & 32.1 & 0.15 \\
$17-F e b-94$ & psqr & 10 & 30 & 10 & 10 & 20 & 0.2 & 0.7 & 30.4 & 3 & 32.1 & 0.19 \\
$17-F e b-94$ & psqr & 10 & 30 & 10 & 10 & 20 & 0.2 & 0.7 & 30.4 & 4 & 32.1 & 0.14 \\
$17-F e b-94$ & psqr & 10 & 30 & 10 & 10 & 20 & 0.2 & 0.7 & 30.4 & 5 & 32.1 & 0.12 \\
17-Feb-94 & psqr & 10 & 30 & 10 & 10 & 20 & 0.2 & 0.7 & 30.4 & 6 & 32.1 & 0.26 \\
17-Feb-94 & psqr & 10 & 30 & 10 & 10 & 20 & 0.2 & 0.7 & 30.4 & 7 & 32.1 & 0.19 \\
17-Feb-94 & psqr & 10 & 30 & 10 & 10 & 20 & 0.2 & 0.7 & 30.4 & 8 & 32.1 & 0.17 \\
17-Feb-94 & psqr & 10 & 30 & 10 & 10 & 20 & 0.2 & 0.7 & 30.4 & 9 & 32.1 & 0.19 \\
& & & & & & & & & & Mean & 32.1 & 0.17 \\
& & & & & & & & & & Std & 0.0 & 0.04
\end{tabular}

Section 5.7.3 $T_{g}$ in $20 \pm 19.9, m_{1}$ in $\pm 0.7, r_{p} \max =0.2$

\begin{tabular}{ccccccccccccc}
\hline Date & Lat & JSZ & ISZ & I $\sigma_{1}$ & $\delta \sigma_{1}$ & $\begin{array}{c}T_{8} \\
\text { m }\end{array}$ & $\begin{array}{c}T_{g} \\
\text { dist }\end{array}$ & $m_{1}$ & $\mathrm{~V}$ & seq & $\sigma_{p k}$ & $p_{\mathcal{C}}$ \\
\hline $17-F e b-94$ & psqr & 10 & 30 & 10 & 10 & 20 & 19.9 & 0.7 & 30.4 & 0 & 31.9 & 0.27 \\
$17-F e b-94$ & psqr & 10 & 30 & 10 & 10 & 20 & 19.9 & 0.7 & 30.4 & 1 & 42.3 & 0.31 \\
$17-F e b-94$ & psqr & 10 & 30 & 10 & 10 & 20 & 19.9 & 0.7 & 30.4 & 2 & 40.7 & 0.31 \\
$17-F e b-94$ & psqr & 10 & 30 & 10 & 10 & 20 & 19.9 & 0.7 & 30.4 & 3 & 31.8 & 0.23 \\
$17-F e b-94$ & psqr & 10 & 30 & 10 & 10 & 20 & 19.9 & 0.7 & 30.4 & 4 & 21.4 & 0.22 \\
$17-F e b-94$ & psqr & 10 & 30 & 10 & 10 & 20 & 19.9 & 0.7 & 30.4 & 5 & 21.3 & 0.21 \\
$17-F e b-94$ & psqr & 10 & 30 & 10 & 10 & 20 & 19.9 & 0.7 & 30.4 & 6 & 30.3 & 0.31 \\
$17-F e b-94$ & psqr & 10 & 30 & 10 & 10 & 20 & 19.9 & 0.7 & 30.4 & 7 & 30.6 & 0.22 \\
$17-F e b-94$ & psqr & 10 & 30 & 10 & 10 & 20 & 19.9 & 0.7 & 30.4 & 8 & 31.6 & 0.21 \\
$17-F e b-94$ & psqr & 10 & 30 & 10 & 10 & 20 & 19.9 & 0.7 & 30.4 & 9 & 31.6 & 0.32 \\
& & & & & & & & & & Mean & 31.3 & 0.26 \\
& & & & & & & & & & Std & 6.4 & 0.05
\end{tabular}

Section 5.7.3 $T_{g}$ in $20 \pm 0.2, m_{1}$ in $\pm 0.7, r_{p}$ max $=0.4$

\begin{tabular}{ccccccccccccc}
\hline Date & Lat & JSZ & ISZ & I $\sigma_{1}$ & $\delta \sigma_{1}$ & $\begin{array}{c}T_{g} \\
\mathrm{~m}\end{array}$ & $\begin{array}{c}T_{g} \\
\text { dist }\end{array}$ & $m_{1}$ & $\mathrm{~V}$ & seq & $\sigma_{p k}$ & $p_{C}$ \\
\hline 14-Mar-94 & psqr & 10 & 30 & 2 & 2 & 20 & 0.2 & 0.7 & 35.2 & 0 & 31.9 & 0.19 \\
14-Mar-94 & psqr & 10 & 30 & 2 & 2 & 20 & 0.2 & 0.7 & 35.2 & 1 & 29.8 & 0.22 \\
$14-M a r-94$ & psqr & 10 & 30 & 2 & 2 & 20 & 0.2 & 0.7 & 35.2 & 2 & 29.8 & 0.14 \\
$14-$ Mar-94 & psqr & 10 & 30 & 2 & 2 & 20 & 0.2 & 0.7 & 35.2 & 3 & 29.8 & 0.17 \\
14-Mar-94 & psqr & 10 & 30 & 2 & 2 & 20 & 0.2 & 0.7 & 35.2 & 4 & 29.8 & 0.15 \\
14-Mar-94 & psqr & 10 & 30 & 2 & 2 & 20 & 0.2 & 0.7 & 35.2 & 5 & 29.8 & 0.16 \\
14-Mar-94 & psqr & 10 & 30 & 2 & 2 & 20 & 0.2 & 0.7 & 35.2 & 6 & 29.8 & 0.23 \\
14-Mar-94 & psqr & 10 & 30 & 2 & 2 & 20 & 0.2 & 0.7 & 35.2 & 7 & 29.8 & 0.21 \\
14-Mar-94 & psqr & 10 & 30 & 2 & 2 & 20 & 0.2 & 0.7 & 35.2 & 8 & 30.0 & 0.13 \\
14-Mar-94 & psqr & 10 & 30 & 2 & 2 & 20 & 0.2 & 0.7 & 35.2 & 9 & 38.8 & 0.30 \\
& & & & & & & & & & Mean & 30.9 & 0.19 \\
& & & & & & & & & & Std & 2.7 & 0.05
\end{tabular}


Section 5.7.3 $T_{g}$ in $20 \pm 19.9, m_{1}$ in $\pm 0.7, r_{p \max }=0.4$

\begin{tabular}{|c|c|c|c|c|c|c|c|c|c|c|c|c|}
\hline Date & Lat & JSZ & ISZ & $\overline{I \sigma} \sigma_{1}$ & $\delta \sigma_{1}$ & $\begin{array}{l}T_{g} \\
\mathrm{~m}\end{array}$ & $\begin{array}{c}T_{g} \\
\text { dist }\end{array}$ & $m_{1}$ & V & seq & $\begin{array}{c}\sigma_{p k} k \\
(\mathrm{MPa})\end{array}$ & $p_{c}$ \\
\hline 22-Mar-94 & psqr & 10 & 30 & 2 & 2 & 20 & 19.9 & 0.7 & 35.2 & 0 & 28.9 & 0.27 \\
\hline 22-Mar-94 & psqr & 10 & 30 & 2 & 2 & 20 & 19.9 & 0.7 & 35.2 & 1 & 38.1 & 0.29 \\
\hline 22-Mar-94 & psqr & 10 & 30 & 2 & 2 & 20 & 19.9 & 0.7 & 35.2 & 2 & 32.6 & 0.32 \\
\hline 22-Mar-94 & psqr & 10 & 30 & 2 & 2 & 20 & 19.9 & 0.7 & 35.2 & 3 & 30.6 & 0.30 \\
\hline 22-Mar-94 & psqr & 10 & 30 & 2 & 2 & 20 & 19.9 & 0.7 & 35.2 & 4 & 18.2 & 0.21 \\
\hline 22-Mar-94 & psqr & 10 & 30 & 2 & 2 & 20 & 19.9 & 0.7 & 35.2 & 5 & 28.3 & 0.31 \\
\hline 22-Mar-94 & psqr & 10 & 30 & 2 & 2 & 20 & 19.9 & 0.7 & 35.2 & 6 & 23.1 & 0.27 \\
\hline 22-Mar-94 & psqr & 10 & 30 & 2 & 2 & 20 & 19.9 & 0.7 & 35.2 & 7 & 27.4 & 0.38 \\
\hline 22-Mar-94 & psqr & 10 & 30 & 2 & 2 & 20 & 19.9 & 0.7 & 35.2 & 8 & 31.8 & 0.36 \\
\hline \multirow[t]{3}{*}{ 22-Mar-94 } & psqr & 10 & 30 & 2 & 2 & 20 & 19.9 & 0.7 & 35.2 & 9 & 28.9 & 0.36 \\
\hline & & & & & & & & & & Mean & 28.8 & 0.31 \\
\hline & & & & & & & & & & Std & 5.1 & 0.05 \\
\hline
\end{tabular}

